\title{
INTEGRATING HUMAN FACTORS ASPECTS INTO PERFORMANCE OPTIMIZATION MODELS OF A SERIAL ASSEMBLY SYSTEM
}

\author{
by \\ Ahmad Sobhani \\ Master of Science, Lulea University of Technology, 2008 \\ Master of Science, Tarbiat Modares University, 2007 \\ Bachelor of Science, Sadjad Higher Education Institute, 2005
}

\author{
A dissertation \\ presented to Ryerson University \\ in partial fulfillment of the \\ requirements for the degree of \\ Doctor of Philosophy \\ in the Program of \\ Mechanical and Industrial Engineering
}

Toronto, Ontario, Canada, 2014

(C)Ahmad Sobhani 2014 



\section{Author's Declaration}

I hereby declare that I am the sole author of this dissertation. This is a true copy of the dissertation, including any required final revisions, as accepted by my examiners.

I authorize Ryerson University to lend this dissertation to other institutions or individuals for the purpose of scholarly research

I further authorize Ryerson University to reproduce this dissertation by photocopying or by other means, in total or in part, at the request of other institutions or individuals for the purpose of scholarly research.

I understand that my dissertation may be made electronically available to the public. 

Integrating human factors aspects into performance optimization models of a serial assembly system

Doctor of Philosophy 2014

\author{
Ahmad Sobhani \\ Mechanical and Industrial Engineering \\ Ryerson University
}

\begin{abstract}
This dissertation investigates the effects of human factors (HF) of the working environment on the performance of an operation system. Poor HF design of the workplace interrupts the balance of the working environment and reduces employees' overall work performance creating a substantial economic burden on organizations. This thesis focuses on integrating HF aspects into performance optimization models of the serial system. For this reason, a modeling framework has been developed for hierarchical consideration of HF consequences at the individual, workstation and system levels. The developed framework provides a road map for the three analytical phases of this $\mathrm{PhD}$ research.

In the first analytical phase, a two-state Markov chain is developed to quantify the connection between Work-related Ill Health (WIH) risk factors (ergonomic conditions in the workplace) and employee health-state in a probabilistic way. Subsequently, an optimization model is developed to minimize the total cost of the assembly system with regard to employee health-related productivity loss. Numerical results indicate that there is between $0.5 \%$ and $8 \%$ difference in the optimal cost of the system with and without including HF effects.
\end{abstract}


In the second analytical phase, a three health-state Markov chain models the connection between HF aspects of the workplace and the employees' work-related productivity and quality variations. Results show between $0.02 \%$ and $32 \%$ increase for the optimal total cost when both employee productivity and quality losses due to poor HF design of the workplace are integrated into the optimization model.

In the third analytical phase, the uncertainty involved in customer demand is considered by developing a two-regime switching model, using a pentanomial lattice. The developed modeling approach investigates the effects of both work-related employee performance variation and demand behavior on the optimal cost of the serial assembly system. Results show that a prediction of the demand distribution throughout the product life cycle is necessary to reduce the over/under cost estimation of the system, due to the stochastic behavior of the demand.

This research opens a new window for considering HF intervention not only as occupational health and safety but also as operation improvement method leading to design safer and more efficient systems. 


\section{Acknowledgements}

I would like to extend my sincerest thanks and regards to all those who supported and encouraged me during my $\mathrm{PhD}$ study.

First, I would like to express my special gratitude to my supervisors Dr. Mohamed Wahab Mohamed Ismail and Dr. W. Patrick Neumann, whose their expert guidance, support, patience and encouragement made my $\mathrm{PhD}$ studies a productive experience.

I would also like to express my special thanks to my examination committee members Dr. Saeed

Zolfaghari, Dr. Cory Searcy and Dr. Ahmad Ghasempoor for their valuable and constructive comments.

I express my gratitude to Dr. Linda Rose for her help, support and valuable suggestions during my PhD studies. I also thank Workplace Safety and Insurance Board of Ontario, Canada for supporting my research.

My deep gratitude is expressed to my mother and father whose love, inspiration and endless encouragement made my years of PhD study an enjoyable and unforgettable experience. They deserve special and heartfelt thanks. I also owe special thanks to my sisters, Anae and Mona, and my brother Milad for their support throughout my life.

Lastly, I would like to specifically thank my beloved wife, Safa, whose love, patience and endless support made this journey bearable for me. 



\section{Dedication}

To Safa my beautiful wife and Aarahn my little hero 



\section{Contents}

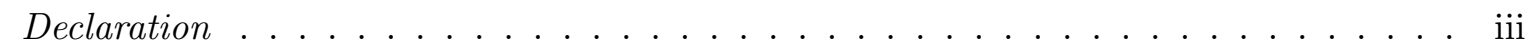

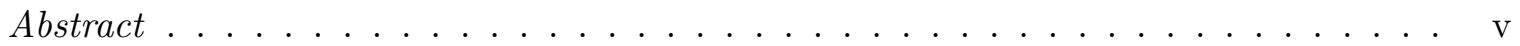

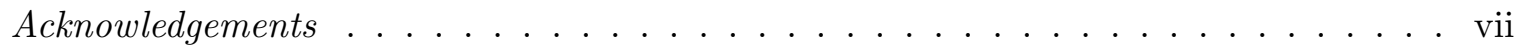

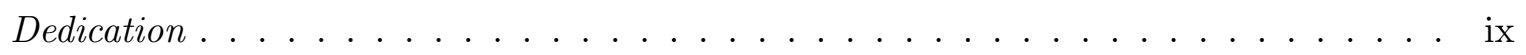

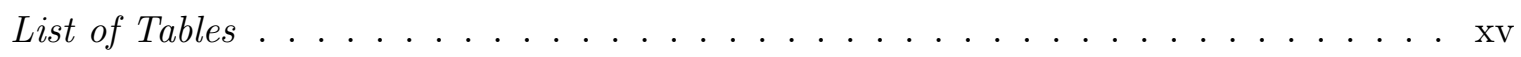

List of Figures . . . . . . . . . . . . . . . . . . . . xvii

List of Appendices . . . . . . . . . . . . . . . . . . xix

\begin{tabular}{lll}
\hline & Introduction & 1
\end{tabular}

1.1 Research Motivation . . . . . . . . . . . . . . . . . . . . . 1

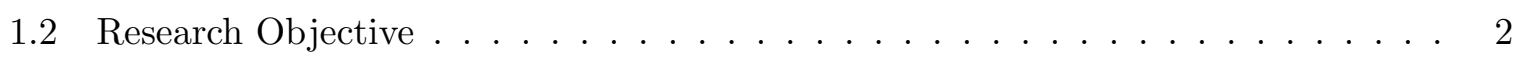

1.3 Research Outline . . . . . . . . . . . . . . . . . . . . . . . . . . . . 3

\begin{tabular}{|lll}
2 & Literature Review & $\mathbf{5}$
\end{tabular}

2.1 Importance of Occupational Health and Safety . . . . . . . . . . . . . . 5

$2.1 .1 \quad$ Economic Consequences of Work-related Health Problems . . . . . . . . . 6

2.1 .2 Musculoskeletal Disorders . . . . . . . . . . . . . . . . . . 8

2.2 Human Factors (Ergonomics) $\ldots \ldots \ldots \ldots$

$2.2 .1 \quad$ Epidemiological Studies $\ldots \ldots \ldots \ldots$

$2.2 .2 \quad$ Ergonomic Intervention Studies $\ldots \ldots \ldots \ldots$

$2.2 .3 \quad$ Ergo-Economic Studies $\ldots \ldots \ldots \ldots \ldots \ldots$

2.3 Operations Management Performance Optimization (OMPO) Models . . . . . . . 22

2.3 .1 Aggregate Planning . . . . . . . . . . . . . . . . 23

2.3 .2 Lot Sizing . . . . . . . . . . . . . . . . . . . . . . 24 
2.3 .3 Misperception of HF Aspects in OMPO Models . . . . . . . . . . . . . . 25

2.4 Summary of the Existing Research Gap $\ldots \ldots \ldots$. . . . . . . . . . . . . . 28

\begin{tabular}{|lll}
\hline 3 & Research Methodology & 31
\end{tabular}

$3.1 \quad$ Research Methodology $\ldots \ldots \ldots$. . . . . . . . . . . . . . . . . 31

3.2 OR Tools and Methods . . . . . . . . . . . . . . . . . . . . . 33

$3.2 .1 \quad$ Logistic Regression $\ldots \ldots \ldots \ldots$. . . . . . . . . . . . . . 33

3.2 .2 Markov Chain . . . . . . . . . . . . . . . . . . 33

3.2 .3 Linear programming $\ldots \ldots \ldots \ldots \ldots \ldots$

3.2 .4 Lattice Approach . . . . . . . . . . . . . . . . . . . . 34

3.2 .5 Dynamic Programming . . . . . . . . . . . . . . . . 35

4 Integration of human factors aspects into operations management performance optimization models: A modeling framework $\quad 37$

4.1 Modeling Approach $\ldots \ldots \ldots$. . . . . . . . . . . . . . . . 38

$4.1 .1 \quad$ Individual Level Sub-Model (ILSM) $\ldots \ldots$. . . . . . . . . . . . . . . 39

4.1 .2 Workstation Level Sub-Model (WLSM) . . . . . . . . . . . . . . . . . . . 41

$4.1 .3 \quad$ System Level Sub-Model (SLSM) . . . . . . . . . . . . . . . . . . . . . . 42

4.2 Illustrative Example $\ldots \ldots \ldots \ldots \ldots \ldots$

$4.2 .1 \quad$ Model Development . . . . . . . . . . . . . . . . . . . . 46

$4.2 .2 \quad$ System Modeling by Integrating HF Effects . . . . . . . . . . . . . . . . 48

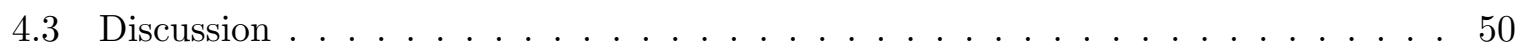

$4.3 .1 \quad$ Benefits of Applying the Framework . . . . . . . . . . . . . . 51

$4.3 .2 \quad$ Framework Challenges $\ldots \ldots \ldots \ldots \ldots \ldots$. . . . . . . . . . . . 52

5 An analytical modeling approach to integrating HF-related productivity loss into a serial system performance optimization models $\quad 55$

5.1 Model Development $\ldots \ldots \ldots \ldots$. . . . . . . . . . . . . . . 56

$5.1 .1 \quad$ Major Steps in the Modeling Approach . . . . . . . . . . . . . . . 58

5.2 Numerical Analysis $\ldots \ldots \ldots \ldots$. . . . . . . . . . . . . . . . . . . . . 62

5.2 .1 Case I: Low Back Pain . . . . . . . . . . . . . . . . . . . . . . 62

5.2 .2 Case II: Work-related Shoulder Disorders . . . . . . . . . . . . . . . . 73 
5.3 Managerial Perspective $\ldots \ldots \ldots \ldots \ldots \ldots \ldots$

6 A modeling approach to integrating employee work-related productivity and $\begin{array}{ll}\text { quality variation into a serial system performance optimization models } & 81\end{array}$

6.1 Problem Description . . . . . . . . . . . . . . . . . . . . . . . . . 82

6.2 Major Steps in The Modeling Approach . . . . . . . . . . . . . . . . . 84

6.2 .1 Step1: Health-State Markov Chain Calculation . . . . . . . . . . . . 85

6.2 .2 Step 2: Calculating The Probability of Making Errors . . . . . . . . . 87

6.2 .3 Step 3: System Performance Optimization Model . . . . . . . . . . . . . . 89

6.3 Numerical Example. . . . . . . . . . . . . . . . . . . . . . . . . . . . . 92

$6.3 .1 \quad$ Major Steps in Evaluating Low Back Pain Risk Factor Effects. . . . . . . 93

6.3 .2 Scenario 1: Individual Influence of WIH Risk Factors. . . . . . . . . . 97

6.3 .3 Scenario 2: Low Back Pain WIH risk Factor Dual Effects . . . . . . . . 100

6.3 .4 Scenario 3: Low Back Pain Odds Ratio Effects . . . . . . . . . . . . . . 101

6.3 .5 Details on Health-related Productivity Loss Variation . . . . . . . . . 103

6.4 Managerial Advantage of The Model . . . . . . . . . . . . . . . . . . . . 104

6.5 Conclusion . . . . . . . . . . . . . . . . . . . . . . . . 108

7 Evaluating the performance of a serial system with regard to stochastic demand and employee work-related performance variation 111

7.1 Serial Production System … . . . . . . . . . . . . . . . . . . . . 113

7.2 Demand Value Estimation Using Lattice Method . . . . . . . . . . . . . . . . . 114

7.3 Markov Chain Health-States . . . . . . . . . . . . . . . . . . . . . . . . . . 117

7.4 Health-related Performance Variation . . . . . . . . . . . . . . . . . . . 119

7.5 Optimization Model … . . . . . . . . . . . . . . . . . . . . . . 121

$7.6 \quad$ Dynamic Programming $\ldots \ldots \ldots \ldots \ldots \ldots$

7.7 Numerical Analysis $\ldots \ldots \ldots \ldots$. . . . . . . . . . . . . . . . . . 125

7.7.1 Scenario 1: Low Back Pain Risk Factor Effects . . . . . . . . . . . . 127

7.7.2 Scenario 2: Stochastic Demand Parameter Variations . . . . . . . . . 133

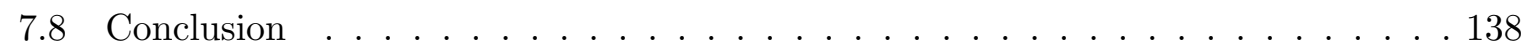


8 Conclusion 141

8.1 Challenges, Limitations and Future Research . . . . . . . . . . . . . . . . . . 142

8.2 Significance of the Developed Modeling Approach . . . . . . . . . . . . . . . 145

\begin{tabular}{lr}
\hline References & 178
\end{tabular} 


\section{List of Tables}

$2.1 \quad$ The rate of direct and indirect costs of occupational injuries in developed countries 7

5.1 Low back pain WIH risk factor measures and parameters used in the Logistic

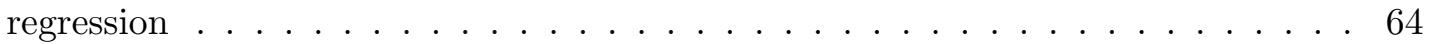

5.2 Examples of WIH risk factor effects $\ldots \ldots \ldots \ldots \ldots$. . . . . . . . 70

5.3 Shoulder WIH risk factor measures and parameters used in the Logistic regression 75

6.1 The value of the system parameters and the corresponding assumptions . . . . . 93

6.2 Low back pain WIH risk factor measures and parameters used in the Logistic regression . . . . . . . . . . . . . . . . . . . . . . . . 95

6.3 Examples of WIH impacts on work-related health, operational and financial aspects 100

6.4 Impacts of productivity loss variation on the assembly line optimum cost items . 104

7.1 The value of the system's parameters and the corresponding assumptions made for the example numerical analysis $\ldots \ldots \ldots$. . . . . . . . . . 126

7.2 Low back pain WIH risk factor measures and parameters used in the Logistic

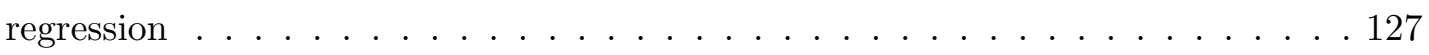

7.3 The examples of work-related health, operational and financial effects of physical WIH risk factors with the growth regime at the beginning of the life cycle (The table includes: physical risk factors' exposure level, the probability of being in-pain, the average excessive fatigue level, the expected error rate of the operators and the cost per unit of the final product for each level of inventory) $\ldots$. . . . . . . . . . . . . . . 130 
7.4 The examples of work-related health, operational and financial effects of psychosocial WIH risk factors with the growth regime at the beginning of the life cycle (The table includes: physical risk factors' exposure level, the probability of being in-pain, the average excessive fatigue level, the expected error rate of the operators and the cost per unit of the final product for each level of inventory) $\ldots$. . . . . . . . . . 131 


\section{List of Figures}

$1.1 \quad$ A general view of the developed modeling framework . . . . . . . . . . . . . . 4

2.1 The mechanism of physical and psychosocial risk factors in developing low back pain, proposed by Kerr 1998 . . . . . . . . . . . . . . . . . . . 12

2.2 The high level procedure of musculoskeletal health improvement . . . . . . . . . 17

$2.3 \quad$ Employee productivity level $\ldots \ldots \ldots \ldots$

$2.4 \quad$ HF vs. HRM approach for human performance improvement $\ldots$. . . . . . . . . . 27

4.1 Developed modeling approach $\ldots \ldots \ldots$. . . . . . . . . . . . . 38

4.2 Individual Level Sub-Model (ILSM) $\ldots \ldots \ldots$. . . . . . . . . . . . 40

4.3 Workstation Level Sub-Model (WLSM) . . . . . . . . . . . . . . . . . . . 42

4.4 System Level Sub-Model (SLSM) . . . . . . . . . . . . . . . . . . . . 43

4.5 An operator posture analysis with different task repetitions . . . . . . . . . . 47

5.1 The behavior of the minimum total cost versus LBP physical risk factor changes 67

5.2 The behavior of the minimum total cost versus LBP psychosocial risk factor

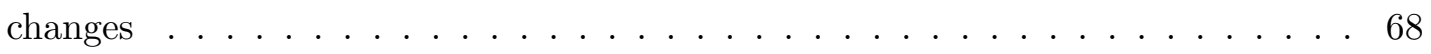

5.3 The behavior of the minimum total cost versus LBP risk factor joint effects . . . 71

5.4 The behavior of the minimum total cost versus changing the odds ratio (ODR) of physical risk factors . . . . . . . . . . . . . . . . . . 73

5.5 The behavior of the minimum total cost versus changing the odds ratio (ODR) of psychosocial risk factors $\ldots \ldots \ldots \ldots \ldots$. . . . . . . . . . . . . . . . .

5.6 Minimum total cost vs. WIH shoulder pain risk factor combinations . . . . . . . 76

6.1 The relative total cost index variation versus Physical WIH risk factor changes . 98 
6.2 The relative total cost index versus Psychosocial WIH risk factor changes . . . . 99

6.3 The relative total cost index variation versus WIH risk factors dual effects . . . . 101

6.4 The behavior of the total cost index of the assembly system versus low back pain WIH odds ratio (ODR) changes . . . . . . . . . . . . . . . . . . 103

6.5 Financial, Operational and Health-related effects of a given WIH risk factor. . . 106

7.1 The first single time-step of the pentanomial lattice . . . . . . . . . . . . . 115

7.2 The valuation of the total cost (present value cost index) versus physical risk factor changes $\left(B_{j 1}=10\right.$ units, the above figures show the model's results with the growth regime at the beginning of the life cycle, the bottom figures show the model's results with the decay regime at the beginning of the life cycle) $\ldots$. . . . . . . . . . 132

7.3 The valuation of the total cost (present value cost index) versus psychosocial risk factor changes $\left(B_{j 1}=10\right.$ units, the above figures show the model's results with the growth regime at the beginning of the life cycle, the bottom figures show the model's results with the decay regime at the beginning of the life cycle) $\ldots . . \ldots . .133$

7.4 The valuation of the total cost versus the mean of the growth regime changes

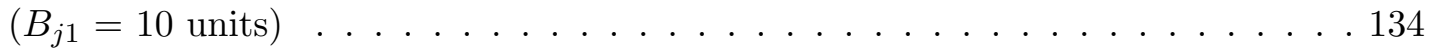

7.5 Lattice based demand estimation versus the mean of the growth regime changes. 135

7.6 The valuation of the total cost versus the volatility of the growth regime changes

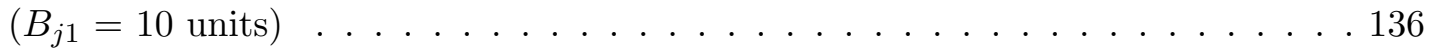

7.7 The valuation of the total cost versus the mean of the decay regime changes $\left(B_{j 1}\right.$

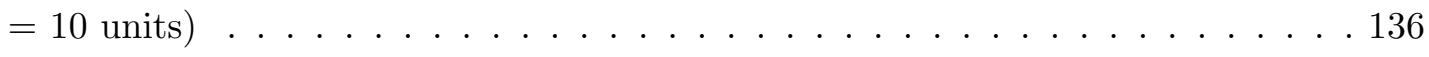

7.8 $\quad$ Expected total cost versus the volatility of the decay regime (Life cycle starts with the growth regime and $B_{j 1}=10$ units) $\ldots \ldots \ldots \ldots$. . . . . . . 137 


\section{List of Appendices}

$\begin{array}{lll}1 & \text { Psychosocial measurement methods } & 149\end{array}$ 



\section{Chapter 1}

\section{Introduction}

\subsection{Research Motivation}

Working Environment (WE) includes physical, psychosocial, and organizational aspects of working conditions that influence work and the involved humans (Rose et al. 2013). The detrimental WE develops occupational illnesses, reducing the employees' overall work performance creating negative economic impacts on companies (e.g., Stewart et al. 2003; Burton et al. 2005; Goggins et al. 2008; Dagenais et al. 2008). Economic costs for Work-related Ill Health (WIH) have been

estimated by some European nations between $2.6 \%$ and $3.8 \%$ of gross national product (EUOSHA 2001). Previous studies show that a major portion of these expenses comes from employee absenteeism and performance variation (productivity and quality loss at work) costs which are usually paid by companies (e.g., Hendrick 2003; Rose et al. 2013). Although human resources are involved in many stages of manufacturing systems, their health-related performance variations have been infrequently considered in Operations Management Performance Optimization (OMPO) models (Neumann and Dul 2010). Operations Management (OM) studies usually use simplifying assumptions regarding human operational characteristics, when human beings are included in evaluating the performance of systems (Boudreau et al. 2003; Neumann and Dul 2010). The performance indicators of systems are usually discussed in terms of cost, profit or throughput. However, OM studies rarely include causes and effects of work-related health problems. This approach usually prevents Human Factors (HF), from playing a critical role in system design and operation planning. Hence, the operation systems are usually sub-optimized 
without considering work-related human illness effects (e.g., Dul et al. 2012).

In contrast to OMPO, HF studies have mostly focused on occupational health and safety aspects (e.g., Kuijera et al. 2007; Kumar and Kumar 2008). HF, used interchangeably with ergonomics, is defined as being concerned with the understanding of the interactions among humans and other elements of a system, in order to optimize human well-being and overall system performance (IEA-Council 2000). However, HF studies seldom have accounted for WIH effects at the system level performance optimization (e.g., Rose et al.|2013). Furthermore, unlike OM models, HF scholars do not usually account for other operational aspects of a system such as demand and their dynamic changing conditions (e.g., David 2005, Rose et al. 2013). Thus, integrating causes and consequences of employee work-related injuries and disorders into OMPO models remains a research gap.

This research proposes a modeling approach capturing work-related performance changes of individuals and their consequent impacts on the overall performance of an operation system for a given level of WIH risks. In particular, this dissertation is focused on investigating effects of WIH-related productivity and quality losses of a serial assembly system's employees when minimizing the total cost of the system. This approach can help operation managers make better decisions and understand long term economic consequences of HF in order to improve the performance of systems, that are both economically and humanly sustainable.

\subsection{Research Objective}

By being aware of the existing gap between $\mathrm{HF}$ and OM research domains, it has been attempted to develop a modeling approach which integrates HF aspects into OMPO models. The purpose of this research is to make a connection between $\mathrm{OM}$ and HF streams by evaluating the impacts of HF aspects on optimizing the performance (total cost) of the serial assembly system. Other features of the operation system such as customer demand, inventory and production costs and Occupational Health and Safety (OHS) expenses are also incorporated into the underlying optimization models.

Figure 1.1 demonstrates a general view of the modeling framework, developed in this research. HF-related elements, operational constraints, technological aspects, and financial features of an operations system are the inputs of the framework. 
In the first step, the modeling framework is focused on defining the employees' health-states based on pain or illness intensity associated with their work-related health problems. Next, their efficiency levels and error rates are measured taking into account the defined healthstates. By applying known relationships between WIH risk factors and the defined healthstates, the modeling framework allows the users to analyze the effect of each risk factor on the employees' performance variations (in terms of productivity and working quality). These variations subsequently change the workstations' outputs. Hence, by assessing the productivity and quality levels of each station, the framework is able to trace the performance fluctuation of the system taking into account the employees' work-related health problems. The performance of the system can be evaluated in terms of cost, profit or throughput analysis. OHS costs are the other HF-related inputs, which are integrated into the framework in order to show the effects of the associated direct and indirect HF costs such as medical care, administrative and replacement expenses.

By considering operational constraints and technological and financial aspects of the system, the modeling framework replicates the behavior of the system more realistically. The examples of the operational constraints include interactions among workstations, number of shifts per day and demand behavior. Number of workstations in the system and machines characteristics are the examples of the technological elements. Some examples of the financial aspects include inventory, production and subcontracting costs. Optimizing the system's output based on the effects of the defined inputs and constraints, enables the modeling framework to consider both effects of HF and non HF elements at the system level analysis.

\subsection{Research Outline}

This $\mathrm{PhD}$ research document consists of eight chapters. Chapter two reviews the HF and OM studies in order to provide a theoretical foundation which is addressing the existing gap between HF and OMPO research streams. Chapter three clarifies the research methodology and introduces the tools that are used for system modeling and data analysis in this study. Chapter four elaborates a generic modeling approach to integrating HF aspects into system performance optimization models. It includes a hierarchical modeling framework to demonstrate how HF effects at the individual level, can have operational and financial consequences at the workstation 


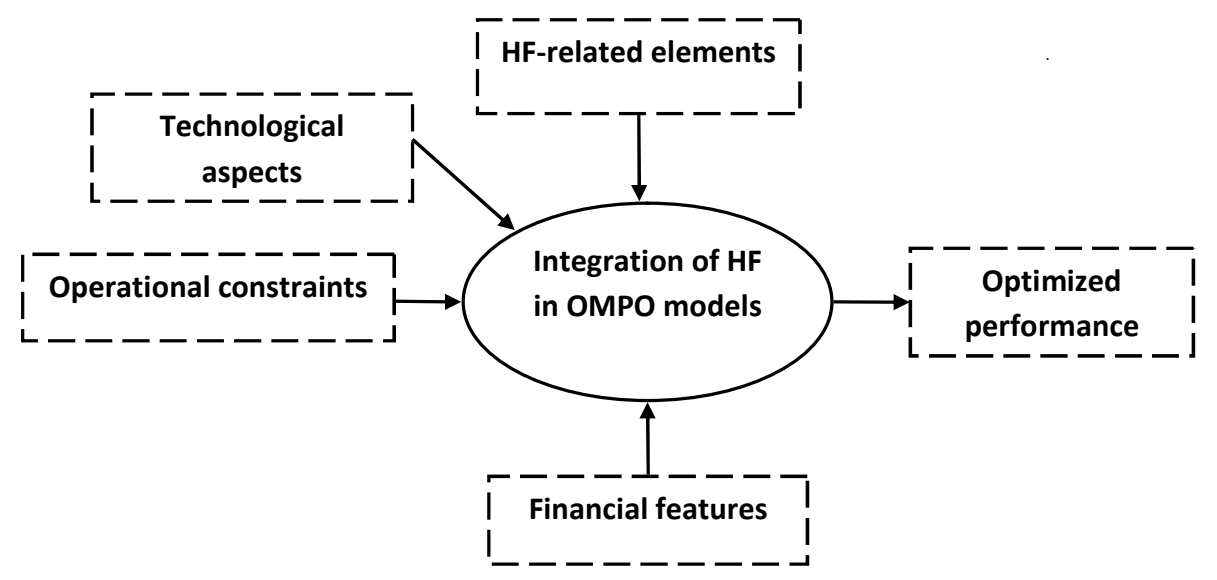

Figure 1.1: A general view of the developed modeling framework

and system levels. Chapter five continues by developing a cost optimization model for a serial manufacturing system including causes and consequences of health-related productivity variation of the involved employees. The results show between $0.5 \%$ to $8 \%$ growth for the minimized cost of the system when HF aspects are integrated into the optimization model. Chapter six discusses the results of minimizing the total cost of the system when the optimization model is extended by including the employees' work-related productivity and quality variation effects. Compared to the optimization model without including HF, the minimized total cost of the system increased between $0.02 \%$ and $32 \%$. Chapter seven evaluates the results of optimizing the serial system's total cost taking into account the stochastic behavior of customer demand and the health-related performance variations of the operators. The numerical results show the necessity of specifying the demand distribution throughout the life cycle in order to replicate the more realistic behavior of the system. Chapter eight concludes the study of the findings and suggests future research priorities. 


\section{Chapter 2}

\section{Literature Review}

This chapter presents a literature review of the Human Factors (HF) and Operations Management Performance Optimization (OMPO) research domains. It aims to help readers become familiar with major relevant elements involved in HF and OMPO research areas and better realize the existing research gap between these two research streams which is the motivation of this research. This chapter starts with exploring the importance of Occupational Health and Safety (OHS). Then, economic effects of work-related health problems are reviewed and the major categories of HF studies are introduced. Subsequently, shortcomings of HF studies are discussed in detail.

The second part of this chapter briefly introduces Operations Management (OM) research area. It focuses on OMPO models and reviews aggregate planning and lot-sizing studies. The existing mis-perception about HF aspects from the OM point of view is briefly discussed.

\subsection{Importance of Occupational Health and Safety}

The OHS field aims to support the safety, health and welfare of humans where working conditions have shown pervasive consequences for the health of employees in developed countries (e.g., Laaksonen et al. 2010; Lindeberg et al.|2011). For example, the annual rate of temporarily disabling occupational morbidity in North America is 40 per 1000 workers-year (Courtney and Webster 1999). Schulte proposed that 15-20\% of injuries among the American working age adults are ascribed to occupational exposures (Schulte 2005). In 2002, occupational health- 
related consensus demonstrates 5,524 fatal work injuries, 294,500 work-related illness and 4.4 million non-fatal injuries in the US (Schulte 2005). By applying conservative assumptions, it has been estimated that occupational injury and disease account for approximately $3 \%$ of all disabilities (Murray and Lopez 1996, Leigh et al. 1997). Nurminen and Karjalainen (2001) reported that $6.7 \%$ of all deaths in Finland in 1996 in age 25-64 were due to occupational health problems. Tompa et al. (2008) also estimated work-related fatal injuries as the eighth cause of death in the US.

According to the Workplace Safety and Insurance Board (WSIB) of Ontario, the annual OHS-related lost time injury rate in Ontario, Canada, was 3.9 per 100 full-time workers covered by WSIB (WSIB 2010a). Moreover, Human Resource and Skill Development Canada (HRSDC) reported that, on average, 1 out of every 46 Canadian workers covered by compensation systems was injured severely enough to miss at least one day of work in 2008 (HRSDC 2010). The statistical report shows that Newfoundland and Labrador, with the occupational fatality rate of 10.7 per 10,000; followed by Alberta with 9 and Ontario with 8.2 are the provinces with the highest occupational fatality rates in Canada. New Brunswick and Prince Edward Island had the lowest fatality rates, 4.1 and 4.5, respectively (HRSDC 2010). The European agency for safety and health at work reported that 146 million lost working hours in the European Union are resulted from more than 4.6 million occupational accidents that happen every year (EUOSHA 2001).

\subsubsection{Economic Consequences of Work-related Health Problems}

Work-related health disorders and injuries create an economic burden due to employee performance variation and the corresponding OHS expenses (e.g., Goggins et al. 2008; Jallon et al. 2011). According to (Leigh et al. 2004) study, $\$ 77.181$ billion was spent on OHS issues in the US while $\$ 60.856$ billion or $78.9 \%$ of these expenses was for non-fatal occupational injuries involving days away from work and $\$ 16.275$ billion or $21.1 \%$ of the expenses, was incurred for fatal cases. In the recent study of Leigh in 2011, $\$ 6$ and $\$ 185$ billion expenses were estimated for the OHS-related fatal and non-fatal injury costs, respectively, in the US (Leigh 2011). Previous studies show that a major portion of these expenses comes from employee absenteeism and presenteeism (productivity and quality loss at work) costs which are usually paid by companies (e.g., Hendrick 2003; Rose et al. 2013). 
At the province level, it was estimated that more than $\$ 6,000$ was spent for each non-fatal work-related injury in Ontario, Canada, while this cost increased to $\$ 492,000$ for each occupational fatal accidents (Marshall 1996). According to the compensation boards of Canada estimations, the total benefit payments per injured person due to occupational health problems were equal to: $\$ 42,792$ in Ontario, $\$ 30,008$ in Newfoundland and Labrador, $\$ 29,887$ in Nova Scotia, and $\$ 28,625$ in New Brunswick (HRSDC 2010). Over the 1996 to 2008 period, compensation payments to injured workers, after adjusting for inflation, have often demonstrated a growing trend in Canada. In 2008, the Workplace Compensation Boards (WCBs) paid $\$ 7.67$ billion for occupational health benefits, which is approximated to $\$ 24,845$ per each new compensated time-loss injury or fatality. In addition, the WCBs paid $\$ 2.03$ billion in health care and vocational rehabilitation payments. Including health care and vocational rehabilitation benefit payment costs, the total direct annual cost of occupational injuries to the Canadian economy was approximately $\$ 9.7$ billion in 2008 (HRSDC 2010$)$.

Recent studies demonstrate that commitments to OHS improvement are often profitable for companies and organizations (e.g., Lanoie and Tavenas 1996; Abrahamsson 2000; Larsson et al. 2007, Rivilis et al. 2008). The profitability mainly originates from improvements in productivity and quality of working, which are defined as indirect costs of work-related injuries (e.g., Helander and Burri 1995; Axelsson 2000; Eklund 2000; Rose 2001; Oxenburgh et al. 2004: Rose et al. 2013). Larsson et al. (2007) showed that companies with superior OHS performance also had faster stock-price growth rates than the market index. Table (2.1) reports the estimates of the direct and indirect costs of occupational injuries and diseases in the sample of developed countries, collected by Tompa et al. (2008).

\begin{tabular}{|c|c|c|}
\hline Country & Base year & Cost as a percent of GDP \\
\hline Great Britain & $1995 / 96$ & $1.2-1.4$ \\
Denmark & $1990 / 92$ & $2.5-2.7$ \\
Finland & 1992 & 3.6 \\
Sweden & 1990 & 5.1 \\
Norway & 1990 & $5.6-6.2$ \\
Australia & $19992 / 93$ & 2.6 \\
Netherlands & 1995 & 2.6 \\
US & 1992 & 3.0 \\
\hline
\end{tabular}

Table 2.1: The rate of direct and indirect costs of occupational injuries in developed countries In Canada, Finland, Sweden, and England, Musculoskeletal Disorders (MSD) cause more 
productivity loss than any other group of occupational diseases (e.g., Punnett and Wegman 2004, Kennedy et al. 2010). As reported by the association of workers compensation boards of Canada, the majority of direct costs of occupational health problems is attributed to MSD (AWCBC 2004). Coyte et al. (1998) also estimated the annual direct cost of MSD in Canada to be $3.4 \%$ of gross domestic product. All these economic consequences demonstrate the huge financial impact of occupational health on organizations. By improving OHS conditions, companies will be benefited from saving the cost of work-related health problems.

\subsubsection{Musculoskeletal Disorders}

Musculoskeletal disorders are the most common work-related health problems in many industrialized countries which include the injuries of the muscles, nerves, tendons, ligaments, joints, cartilage, or spinal discs (e.g, Denis et al. 2008; Da Costa and Vieira 2009; Lin et al. 2011; Oh et al. 2011). These injuries often cause a great deal of pain and suffering among afflicted workers with a more gradual or chronic development in back, neck, shoulders and upper limbs (e.g., Rijn et al. 2010; Driessen et al. 2012). The incidence of MSD is rising in Canada, United States, and Europe because of age, lifestyle factors and work intensification (e.g., Heneweer et al. 2011). On average, $85 \%$ of the population sometimes during their lifetime experienced MSD (Arnetz et al. 2003). MSDs are also among the most common lost-time/non-lost time injuries (e.g., Dagenais et al. 2008; Cote et al. 2009). In the US alone, more than 600,000 workers have MSD resulting in days away from work each year (Baldwin 2004). It was reported that more than $40 \%$ of all compensated claims in Ontario, Canada, were registered because of MSD problems (Choi et al. 1996). In 1994, there were 490,094 low back illnesses were reported causing at least one day away from work (Tompa et al. 2008). According to the WSIB annual report, upper limb injuries accounted for about 30\% lost time claims in Ontario in 2006 (WSIB 2006). Moreover, MSD accounted for over $28 \%$ of compensation costs in Ontario, Canada, during 1996-2002 (WSIB 2006). It is estimated that $15 \%$ to $25 \%$ of all compensation claims were attributed to work-related back disorder claims (Arnetz et al. 2003).

MSDs are the most costly occupational health problems. For example, in the United States, direct costs due to musculoskeletal pain or discomfort exceed $\$ 52$ billion annually Da Costa and Vieira 2009). Back disorders included 14.4\% of all claims between 1992 and 2000 in the US incurring $\$ 1.5$ billion in medical and health care costs (Silverstein et al. 2002). MSD- 
related costs are more than compensation and health care costs (direct costs). They also include the indirect costs such as decreasing on-job productivity and quality services (Tompa et al. 2008). The health-related economic consequences of MSD problems in developed nations demonstrate the necessity of OHS investigations. Occupational HF scholars aim to elaborate this investigation in ways to consider a) factors affecting on health and safely of the employees; or b) the design of work environments in order to make them compatible with the health and safety needs and the people's abilities; or c) the costs and benefits of improving the employees' health and safety conditions.

\subsection{Human Factors (Ergonomics)}

According to International Ergonomics Association (IEA) definition "Human factors, ergonomics, is the multidisciplinary scientific discipline involved in the understanding of interactions among humans and other fundamentals of a system, and the profession that applies theory, data and techniques to design systems. It aims to optimize human well-being and overall system performance" (IEA-Council 2000). A literature review shows that scholars in the occupational HF field usually conduct research in a) identifying the important work-related health problems and evaluating their associated physical, psychosocial, social and biological work-related ill health (WIH) risk factor consequences; b) applying the HF information to the design of tools, machines, workplaces and systems to develop a safer, comfortable working environment; and c) assessing economic consequences of HF improvement. For the purpose of this thesis, occupational HF studies are classified into three major categories, which are referred as epidemiological, ergonomic intervention and ergo-economic studies. These categories are briefly discussed in the following.

\subsubsection{Epidemiological Studies}

Epidemiological research studies causes and effects of disease conditions in the target populations (e.g., Hoy et al. 2010). The contribution of epidemiological studies in HF field includes the analysis of the relationship between working environment aspects and work-related health hazard risks (e.g., Meerding et al. 2005; Driessen et al. 2010). These types of studies usually follow a structure and systematic identification approach to assess work-related ill health risk 
factors, including physical and psychosocial aspects. They evaluate the effects of these risk factors from the health and safety point of view (e.g., Vieira and Kumar 2007; Kucera et al. 2009).

In practical terms, the results of epidemiological analysis help occupational HF users: a) be aware of work-related health hazards and evaluate the likelihood of the associated occupational illnesses or injuries occurring; b) identify who may be at risk at the workplace (e.g., employee); c) make decisions about the adequacy of existing HF-related control measures; d) prioritize health hazard control measures and provide information to reduce the risks.

A literature review of epidemiological analysis in HF field demonstrates that they usually have been focused on health analysis at the individual level. They have rarely investigated the performance-related consequence of occupational health problems and associated WIH risk factors at the workstation and system levels. This thesis aims to take the advantage of epidemiological research approach to identify the important work-related health problems. Then, it focuses on the corresponding WIH risk factors to better understand how these health hazard risks contribute to human performance variation resulting from the occupational health problems.

\section{Work-related Risk Factors}

A risk factor is defined as a factor that results in increasing the chance of a disease. Differences in the risk for neck, back and upper extremity complaints are related to differences in working conditions (Winkel and Mathiassen 1994, Cole and Rivilis 2004). Epidemiological studies show that the most WIH risk factors can be clustered into two main groups:

- Physical risk factors. Some examples include repetitiveness of tasks, handling heavy loads, vibration, percent of time in force, spine compression, peak spinal loads, peak load handled, peak lumbar shear, extreme hand and arm postures, strained hand and arm movements, sudden muscular effort, short work cycles, and task invariability (e.g., Bernard 1997; National Research Council 2001; Devereux et al. 2002; Neumann 2004; Coenen et al. 2013).

- Psychosocial risk factors. Some examples include social support, decision making latitude, job control, job satisfaction, co-worker support, supervisor support, psychosocial work 
demands, working overtime, job stress, mental demand and mental pressure (e.g., Bongers et al. 2002; Kumar and Kumar 2008; Ramond et al. 2010).

There is a complex mechanism for psychosocial risk factors in developing work-related MSD which have been investigated by occupational epidemiologists and ergonomists (e.g., Moon and Sauter 2005, Haukka 2010). According to National Institute of Occupational Health and Safety (NIOSH 1993), psychosocial demands in the workplace may generate an increased involuntary muscle tension, which is intensifying the negative effect of physical risk factors (biomechanical strain) in developing musculoskeletal disorders. Different scientific based models were proposed by scholars to indicate the mechanism of psychosocial risk factors (e.g., Kerr 1998). For instance, Moon and Sauter (2005) developed a scientific evidence based conceptual model visualizing a direct path between work organization (working tasks) and work-related psychosocial loads. According to this model, in general, psychosocial risk factors have a dual contribution in developing work-related disorders. First, perceived psychosocial load can produce muscle tensions, which increase biomechanical strain generated due to work-related physical demands. This increased strain raises the chance of developing work-related MSD. Second, psychosocial risk factors can also moderate the relation between the work-related biomechanical strain (physical risk factor effects) and the symptoms of the work-related MSD. Haukka (2010) explained the second contribution by suggesting that "the relationships between biomechanical strain and the development of MSD are mediated by complex cognitive processes that involve the detection and sensation of symptoms and their attribution to the musculoskeletal system." Kerr (1998) articulated the involvement of psychosocial risk factors in developing work-related low back pain in an automotive company in Canada, shown by Figure 2.1. According to the Kerr model, the appearance of work-related low back pain is due to the result of direct and/or indirect effects from the exposure of either psychosocial or biomechanical risk factors.

There are also other risk factors that can be categorized as the social and biological factors, depending on age, sex, weight, height, body mass, children, marital status, smoking, training, job title etc. (e.g., Kerr et al. 2001; Buckle and Devereux 2002). A system performance evaluation approach usually focuses on the elements of the system that are commonly related to working environments. For this reason, physical and psychosocial elements are considered as the main work-related risk factors in evaluating the performance of the system. This $\mathrm{PhD}$ research aims to develop system performance optimization models, including both physical and 


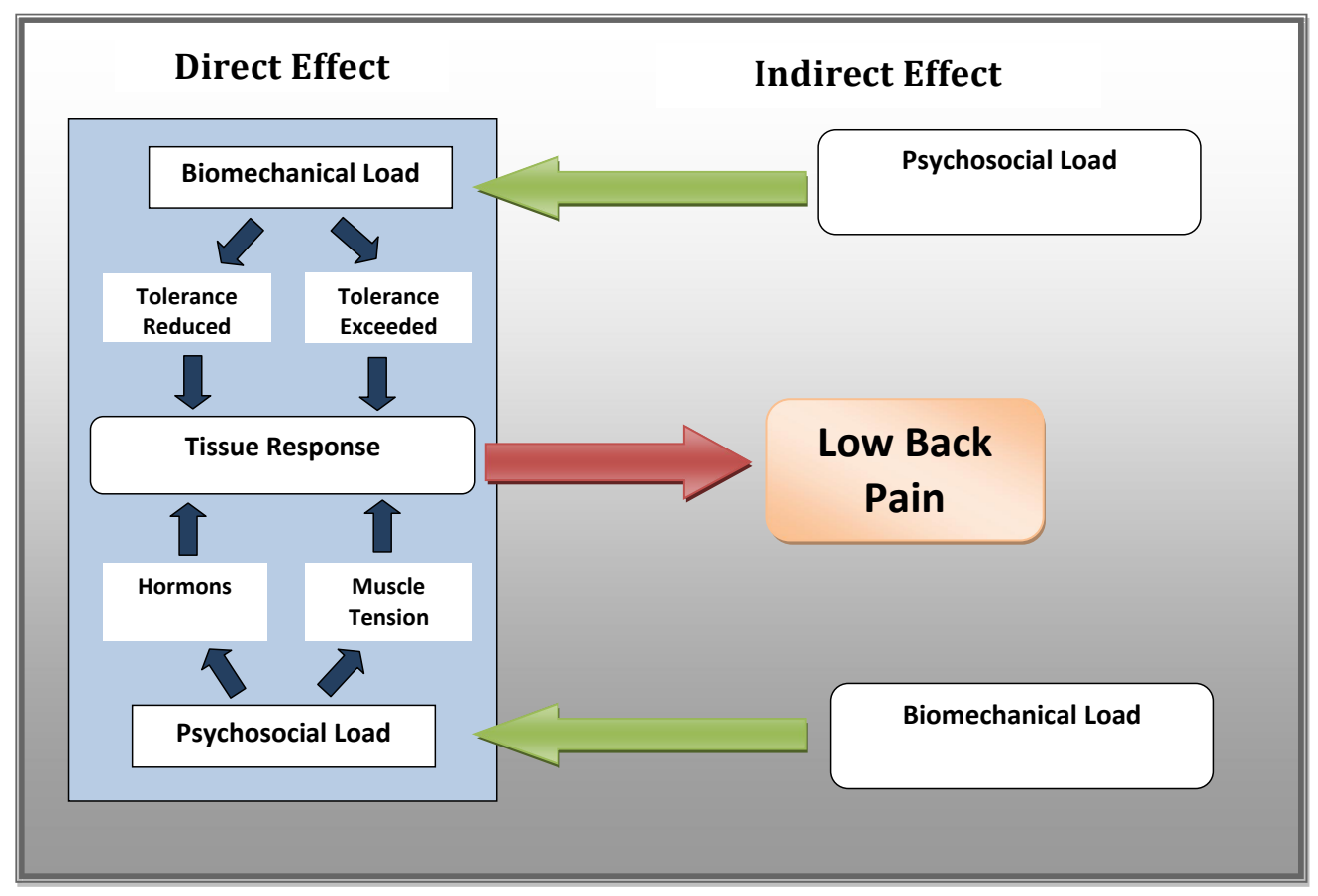

Figure 2.1: The mechanism of physical and psychosocial risk factors in developing low back pain, proposed by Kerr 1998

psychosocial WIH risk factor effects.

Epidemiological studies usually draw on experimental research designs to collect and analyze the association among WIH risk factors and hum health-related outcomes. These research design approaches are briefly introduced in the following.

\section{Prospective Study}

A prospective (cohort) study is an analytical measuring approach in which individuals are observed over time periods to determine the association between suspected WIH risk factors and the occurrence of certain occupational health problems (e.g., Coenen et al. 2012). Two groups of subjects are usually selected. One group has been exposed to the WIH risk factors while the other has not. To determine the contribution of the WIH risk factors, this study design compares the incidence rates of the health problems between the groups (e.g., Garg et al. 2013).

One of the advantages of the prospective study is that it helps applicants reduce the "recall 
errors" in assessing the causal association between a risk factor and the work-related human health outcomes (Glenn 2005). A prospective study is a longitudinal observation. Hence, it is easier for the applicants to determine the casual relation between a certain health outcome and the exposure of a suspected risk factor than such as case-control and cross-sectional designs, where the chronological sequence is more difficult to determine. This type of study also permits the users to directly calculate the incident rate for both groups in order to determine the risk or rate ratios of injury (Glenn 2005). Furthermore, prospective study enables the users to investigate different outcomes simultaneously. For many occupational health problems, there is a relatively low incidence rate of injury and long time periods are needed to collect associate data in perspective study. Therefore, the prospective research design users usually must investigate a large sample of the population and reassess risk factor exposures on a frequent basis in order to bring enough cases for valid statistical comparisons (Glenn 2005). Hence, prospective (cohort) study is a costly experimental design.

\section{Case-Control Study}

Case-control study is an observation research design widely used to identify WIH factors that may contribute to a specific occupational health condition by comparing two groups (a binary approach). The first one includes subjects who have the occupational health problems (the "cases") while the members of the second group do not but are otherwise similar, the "controls" (Armenian 2009). To determine the association between a WIH risk factor and the human health condition, the users of this research design are looking back in time to determine the proportion of each group exposing under the WIH risk factor and then compare them (e.g., Kerr et al. 2001).

The case-control is usually involves a smaller sample size for collecting data. Hence, being less costly and less time consuming, compared to the prospective approach, are the most important advantages of the case-control research design method. A case-control study is usually involves in a non-randomized trial to select the "case" members in the process of collecting data. Hence, the users of this method in epidemiology usually consider the relative risks of WIH risk factors in the occurrence of occupational health problems which are determined according to the comparison between the case and the control groups (King and Zeng 2002). 


\section{Cross-Sectional Study}

Cross-sectional studies use a research design in which a single sample of population is considered, without regard to the sample's members have a specific health condition or they are exposed to specific WIH risk factors (e.g., Oh et al. 2011). This research approach provides a "snap-shot" of the frequency and characteristics of a health problem in a study population at a particular point in time. This research design aims to determine the prevalence of the under-study occupational health problems in the presence of different WIH risk factors. Like the case-control approach, this type of research design study incurs less cost and needs less time than the Prospective study.

This $\mathrm{PhD}$ thesis aims to make a connection between WIH risk factors and human workrelated health states. This connection helps the research to test the contribution of each risk factor at the system level analysis. Considered as a generic modeling approach, the models developed in this study are able to apply WIH risk factor data, from any of the experimental design methods reviewed in the above sections.

HF scholars use different tools to measure work-related health outcomes and associated physical and psychosocial WIH risk factors. Apart from clinical examinations, health research centers have developed different means to determine the occurrence of occupational health problems according to their symptoms. Since this $\mathrm{PhD}$ research is based on defining human work-related health states and identifying MSD risk factors, in the following some of the existing HF health evaluation tools are briefly reviewed. The outputs of these tools are considered in the research in order to define employee health-states. In practice, the users of the models developed in this research would apply the health evaluation tools to determine employee work-related health status and WIH risks in their operation systems.

\section{SF-36 Questionnaire}

The SF-36 is a multi-purpose, short-form health survey. It includes an 8-scale report of functional health and well-being scores as well as psychological, physical and mental-based health summary measures and a preference-based health utility index. The SF-36 has proven useful in surveys of general and specific populations, comparing the relative burden of diseases. In 1996, Version 2.0 of the SF-36 (SF-36v2) was introduced, to correct deficiencies identified in 
the original version. Those improvements are documented in the SF36v2 user's manual (Ware et al. 2000). Both SF36 and SF-36v2 have been used in many HF studies in order to measure the overall health status of employees.

\section{Nordic Musculoskeletal Questionnaire (NMQ)}

The Nordic Musculoskeletal Questionnaire was developed by the Nordic council of ministers in order to investigate low back, neck, shoulder and general work-related MSD complains. NMQ comprises general information about age, weight, height, smoking habit, work experience and shift type and also questions about problems on the whole body and specific body parts (neck, shoulders and lower back). A body "map" was also used to make it easier for workers to visualize their problems in each body area (Dawson et al. 2009).

\section{NOISH}

NOISH equation is an ergonomic measurement tool that was developed by the National Institute for Occupational Safety and Health (NIOSH) in 1981 and was revised in 1991. It helps safety and health practitioners evaluate lifting demands (Waters et al. 1993). These lifting guidelines are being widely used to provide an empirical method for evaluating manual lifting tasks. NIOSH also is able to assess the potential hazards of manual lifting performed in the workplace.

\section{Job Content Questionnaire(JCQ)}

The JCQ is a self-administered instrument which was designed to measure social and psychological characteristics of jobs. The best known psychological scales used by this tools are: (a) decision latitude; (b) psychological demands; and (c) social support. These scales are used to measure high demand, low-control, low-support model of job strain development from psychosocial point of view. All these scales can be used for micro level, job-characteristic analytical purposes, such as assessing the relative risks of individuals' exposures to different work situation to predict job-related illness development, psychological distress, coronary heart disease, musculoskeletal disease, and reproductive disorders (Karasek et al. 1998).

Apart from the introduced tools, here, other instruments are used in HF studies in order to evaluate WIH risk factors. Watbak, Stool, Rula are the examples analyzing the posture of 
employees during performing their jobs (e.g., Norman et al. 1998). These tools usually break down jobs into different tasks and analyze the body posture of employee for the assigned tasks.

\subsubsection{Ergonomic Intervention Studies}

Studies in this category aim to implement ergonomic interventions based on the evaluation of WIH risk factors at a workplace (e.g., Tompa et al. 2008; Neumann et al. 2009). They usually seek ways to reduce the WIH risk factor exposures by redesigning the processes, tools, and equipment used at the workplace (e.g., Tuncel et al. 2008; Tompa et al. 2008; Rivilis et al. 2008). Figure 2.2 demonstrates a high level general procedure of MSD improvement in a real working environment by applying appropriate ergonomic intervention, introduced by Westgaard and Winkel (1997). This procedure starts with collecting occupational HF information from epidemiological studies to identify the major factors affecting MSD in a particular working environment. Other sources of HF information can be gathered from laboratory studies accomplished by previous scholars or HF-related experimental analysis designed for an specific working environment. Using these resources can help practitioners develop ergonomic guidelines and designed interventions to evaluate ergonomics indicators and assess the effectiveness of the ergonomic interventions via reducing the negative effects of physical and psychosocial WIH risk factors.

As a general view, ergonomic interventions are usually classified into 2 major groups: a) preplanned ergonomic interventions: these types of ergonomic improvements are applied according to a pre-planned intervention concepts focusing on general workplace risk factors. They are parts of scientist studies or economic consulting (Tompa et al. 2008) b) participatory ergonomic interventions: these types of interventions are usually involved in operating system improvements in terms of management action and employee contributions. They include systematic and participatory activities to make the employees reach ergonomic intervention objectives (e.g., Ramond et al. 2011).

This PhD research considers HF effects on the the performance of operation systems. Therefore, the results of the developed models can help managers better understand how ergonomic condition of a workplace affects the operation of the systems. Managers are able to select the optimum ergonomic solutions according to their associated advantages, which can be financially predicted by the models developed in this research. 


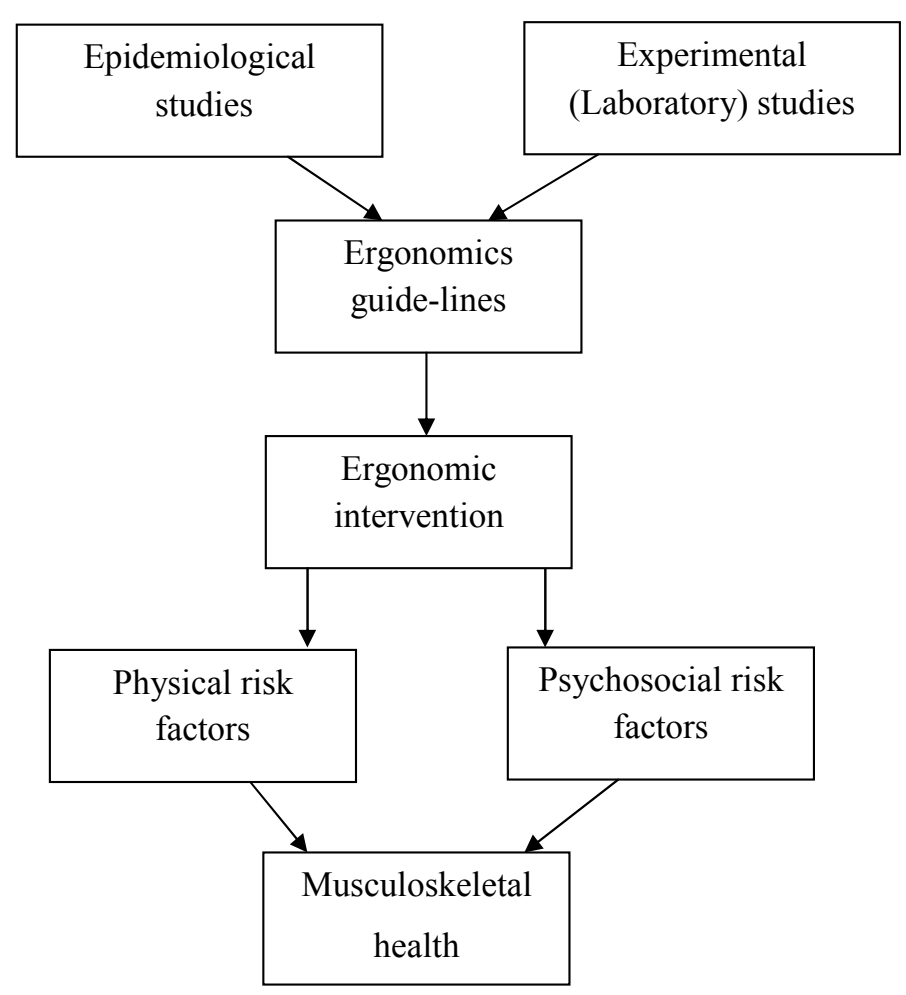

Figure 2.2: The high level procedure of musculoskeletal health improvement

The benefits and effectiveness of ergonomic interventions have always been a question (Tuncel et al. 2008). Especially from a business perspective, the cost effectiveness of an ergonomic intervention has been often unclear (e.g., Morse et al. 2009). This is mainly due to the intricate nature of the investigated costs making insufficient related information for decision making analysis (e.g., Rose et al. 2013). Therefore, HF-economists put efforts to justify ergonomic interventions.

\subsubsection{Ergo-Economic Studies}

The definition of HF implies that the ergonomics perspective has both a social goal (human wellbeing) and an economic goal (overall system performance). However, HF is often unfamiliar and vague for decision makers. Most of occupational HF studies primarily deal with the wellbeing goal of ergonomics (e.g., Gold et al. 2009). They usually work on the basis of health and safety concepts (Dul and Neumann 2009). Therefore, ergonomics is primarily seen by the 
business world as a health issue. To this end, several studies have started to justify the costs and benefits of HF investigations, at the organizational level, in the past two decades. They show that the investigation of HF aspects can improve productivity and quality (e.g., Beevis 2003: Hendrick 2003; MacLeod 2003; Seeley and Marklin 2003; Lahiri et al. 2005). Some of these studies aimed to address the possible direct and indirect costs of poor HF design while others developed economic methods in order to evaluate ergonomic interventions. In the following sections, the possible direct and indirect OHS costs and benefits are reviewed. Then, recent economic-models evaluating ergonomic interventions are briefly introduced.

\section{Direct and Indirect Costs}

OHS-related expenses include both direct and indirect costs. This categorization depends on analyst goals in conducting their projects and accessible sources. Therefore, some cost items are classified as direct costs in some studies while they maybe categorized as indirect costs in other studies. The direct OHS costs are involved in the health related costs which can be easily linked to production and labor. They usually include:

- Absenteeism costs. Costs paid to an injured person during his or her absence are referred as absenteeism costs. Sickness wages, payroll taxes, fringe benefits and medical and rehabilitation expenses are the examples of absenteeism costs which are jointly paid by employers and employees (e.g., Andersson 1992; Hendrick 2003; Siddharthan et al. 2005).

- Wage replacement. An injured employee may incur damages to the employer facilities. The costs returning to repair and replace these damages are defined as wage replacement cost (e.g., Hendrick 2003; Oxenburgh and Marlow 2005).

The indirect costs include all overhead expenses. These costs are estimated to be between 2 to 10 times of the direct costs (e.g., Leigh et al. 2004). They are usually clustered into 2 main categories, replacement costs and presenteeism costs:

- Replacement costs. Replacement costs are identified as all overhead costs incurred to replace a new employee with the injured one. They include:

- Acquisition costs. Recruiting, selecting, hiring, and induction expenses of the new 
employees are usually referred as acquisition costs (e.g., Hendrick 2003, Siddharthan et al. 2005; Sun et al. 2006).

- Development costs. These costs are paid by employers to train the replacement workers to the level needed or to enhance an individual skills. A specific cost for orientation, off the job training includes salaries for persons providing training and information material and travel (Andersson 1992; Hendrick 2003; Tompa et al. 2008).

- Separation costs. They include severance pay costs and vacant position costs. Severance pay cost is the amount of prepaid compensation given to an employee upon leaving the company and vacant position costs returns to those incurred till the company can replace a new worker. These kinds of costs are usually backing to the lower production rate and outsourcing the production (e.g., Andersson 1992 , Hendrick 2003).

- Insurance premium cost. This cost paid by a company to buy the insurance is also frequently categorized as an indirect OHS cost which usually depends on the type of the industry and the company's OHS records (Jallon et al. 2011).

- Investigation costs. When an accident happens, an employer must allocate human and financial resources to complete the associated legal and administrative documents, set up and monitor the file, enter data in the accident registry, compile accident statistics, issue a report, etc. All these activities incur expenses referred as investigation costs (Jallon et al. 2011).

- Presenteeism costs. Presenteeism costs are usually defined as on-job productivity loss (e.g., Heuvel et al. 2010). Those have been identified as the major costs incurred to employers with respect to the reduction of employees on-job productivity and increased quality deficits (e.g., Axelsson 2000; Meerding et al. 2005; Oxenburgh and Marlow 2005).

Figure 2.3 shows the absenteeism and presenteeism effects on human performance. It is supposed that a skilled employee is $100 \%$ productive while he/she does tasks at the workplace. But, as time continues, the negative impacts of WIH risk factors cause the employee's productivity level to decrease and his/her error rate to increase because of health problems such as work-related low back, neck and shoulder pains etc.. At time $t_{1}$, the employee finally leaves work 
due to the WIH problems and the employer is incurred absenteeism costs. As the employee's WIH issues resolve he/she may return to work before with a capacity lower than $100 \%$ (Time $t_{2}$ in Figure 2.3). In this case, there is a second period of under performance at work (a lower efficiency and a higher error rate), as the worker gradually returns to $100 \%$. This health-related production loss was explained in previous studies (e.g., Butler et al. 2006, Alexopoulos et al. 2008). In general, employers pay extra costs such as reworking, over-time and back order costs due to a health-related lower performance of the employee.

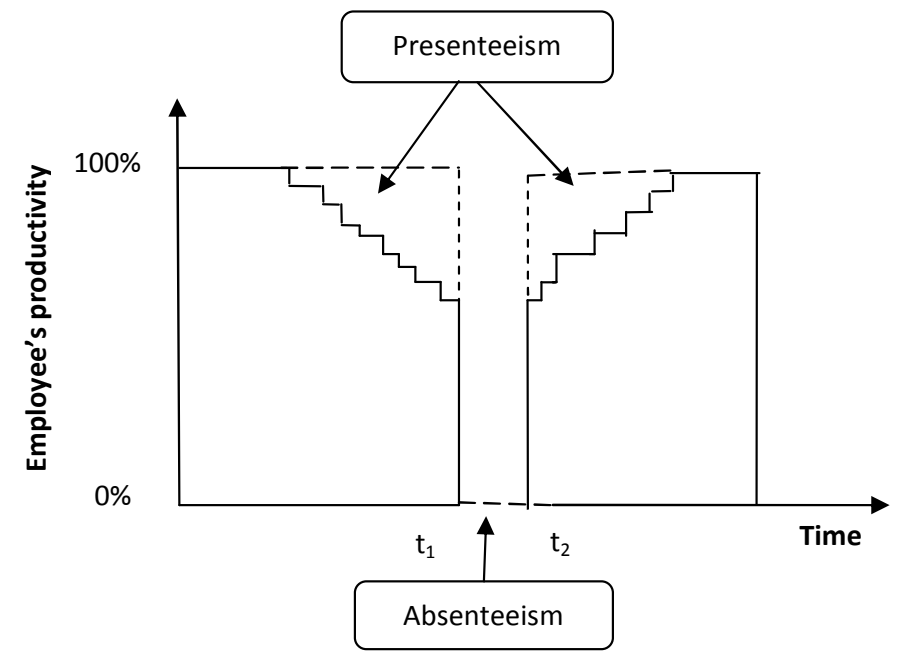

Figure 2.3: Employee productivity level

\section{Ergo-Economic Models}

While there is much discussion about the need to cost-justify ergonomics (Beevis 2003; Hendrick 2003 Lahiri et al. 2005), few actual tools are available for the ergonomics practitioner for the evaluation of HF investment worthiness. According to Rose et al. (2013), the existing ergoeconomic models can be categorized into the following three groups:

Suggestive Models Combined with Investment Analysis These models aim to identify possible HF related problems through companies and suggest different solutions in terms of ergonomic interventions. They usually estimate the rough costs and benefits of the proposed interventions. For example, the balloon model, proposed by Johanson and Johraon (2001), 
clusters the advantages and disadvantages of OHS aspects and makes suggestions for future ergonomics interventions. In this tool, input data are given in form of personnel and worksheet data, monetary costs and benefits data associated with an intervention. A characteristic feature is the brainstorming approach utilized to highlight and identify the costs associated with deficiencies in the workplace and the corresponding benefits associated with interventions.

The Return on Health, Safety and Environmental Investments (ROHSEI) is another model which was created by Linhard (2005). It is a supporting tool for use by OHS and environmental professionals to provide a comprehensive look at ergonomics investments. It aims to make a comprehensive understanding of the impact of ergonomic investments on organization's health, safety and environmental goals as well as its business and financial goals. In this model, HFrelated problems are investigated and different possible ergonomic solutions are compared in terms of intervention measures. The ROHSEI also follows a team approach by integrating health and safety metrics such as compensation cost and lost days injury into financial assessment models. This model will show the consequences of alternative ergonomic investments in terms of return on investment payback period etc.

Workplace Risks Analyzer Models These models start analysis with assessing workplace hazard risks and calculate their economic impacts. MAWRIC is an example of the models involving in work-related risk identification, risk assessment and the estimation of corresponding costs. It gives the users ergonomic suggestions to reduce the negative effects of workplace risk factors (Rose 2001). In the first step, MAWRIC assesses the possible health-related risks associated with jobs at workstations. Then, it classifies the chance of the risks and evaluates the corresponding possible financial and health and safety consequences. Finally, MAWRIC estimates the $\mathrm{HF}$-related costs of jobs according to their risk assessment. With the results from the jobs' risk assessment and associated cost estimations, MAWRIC applies a decision making process to realize whether the risk levels of jobs are economically acceptable.

Ergonomic Investments Analysis Models These HF-economic models only focus on costbenefit analysis of ergonomic interventions. The net-cost model presented by Lahiri et al. (2005) is an example estimate costs and benefits of the ergonomics investments before and after an intervention. By performing cost-benefit analysis, the model justifies ergonomics investments, 
evaluates different ergonomic solution alternatives and ranks their profitabilities. Another well established method for the cost-benefit analysis of ergonomic investment measures at the company level is the productivity model developed by Oxenburgh et al. (2004). This model was designed for workplace health problem evaluation by assessing the costs and benefits of ergonomic investments.

The literature review of ergo-economic models indicates that they are focused on the cost impact of proposed ergonomic changes. They neither examine workplace risk factors contributions at the system level nor constitute probabilistic or stochastic process in addressing HF economic effects (e.g., Lahiri et al. 2005). They also fail to capture the long term dynamic system characteristics. These models usually tend to use a snap-shot approach, by running the cost-benefit analysis after and before an ergonomic intervention (e.g., Linhard 2005; Oxenburgh and Marlow 2005; Rose 2001). This thesis aims to develop models including HF aspects at the system level evaluation. The models are aimed to address the limitations, discussed above.

\subsection{Operations Management Performance Optimization (OMPO) Models}

Operations Management $(\mathrm{OM})$ is defined as the management of operational activities to provide goods and services. OMPO models contain analytical methods of problem-solving and decisionmaking. These models are used in many areas of manufacturing systems and supply chain management including Capacity planning (e.g., Gademann and Schutten 2005; Huang and Ahmed 2010), Inventory control (e.g., Sajadieh et al. 2009; Tsou 2009), Resource assignment (e.g., Bravo and Gonzalez 2009; Novoa and Storer 2009), Flexibility measures (e.g., Wahab and Lee 2008; Hallgren and Olhager 2009), Production strategy and Throughput analysis (e.g., Matsubayashi and Yamada 2009, Schultz et al. 2010), Supply chain system (e.g., Pavitsos and Kyriakidis 2009; Wahab and Jaber 2010), and Lot sizing (e.g., Brahimi et al. 2006). They optimize the performance of systems while considering resources involved in their operations (e.g., Ahmed and Sahinidis 2008).

OM scholars also apply OMPO models to evaluate the efficiency of production/service processes involved in systems (e.g., Wahab and Jaber 2010). They aim to organize the employment of resources in order to maximize the systems' efficiency and keep their production times and 
costs to a minimum level. A review of OM studies shows that human and technology aspects have been defined as important examples of the resources that can play critical roles in establishing systems with a sustained long term performance (e.g., Das and Teng 2000; Lockett and Thompson 2001; Wright et al. 2001; Wan et al. 2011). However, a literature review shows that OMPO models infrequently include workforce capability variations and quality deficits due to human work-related health problems, even while several HF studies prove that health problems can reduce the productivity rate of employees (e.g., Meerding et al. 2005; Iverson et al. 2010). When OMPO studies include human aspects, they usually assign a fixed value to employee performance and do not investigate WIH risk factors and their effects on the employee capabilities that influence the performance of systems (e.g., Al-e hashem et al. 2013).

Aggregate planning and lot sizing are the two popular examples of production planning methods which are widely used by OM practitioners and scholars. They are considering productivity and availability levels of an operation system resources such as machine production rates and workforce levels when the users optimize the performance of the system.

\subsubsection{Aggregate Planning}

Aggregate production planning is accomplished by utilizing human and equipment resources of an operation system (e.g., Mirzapour Al-e hashema et al. 2011). This method is involved in determining production, inventory, and workforce levels simultaneously to meet variable demand requirements over a given period of planning that usually ranges from six months to one year (e.g., Meybodi and Foote 1995, Fung et al. 2003, Wang and Liang 2005). Aggregate production planning is developed based on the existence of an aggregated demand for master production schedule of the operation system. Once the aggregate production plan is generated, constraints are imposed on detailed production scheduling process determining the specific quantities to be produced. The plan must take into account various ways to deal with demand uncertainties as well as the costs associated with them. The following four approaches are usually considered in aggregate models to manage demand variation:

- Change the workforce levels by hiring and firing. This approach allows the models to plan with different production rates during production horizons. The important constraint in this approach is imposed by union regulations or human resource management policies to limit the excessive use of hiring and firing of employees (e.g., Da Silva et al. 2006). 
- Introducing overtime or outside subcontracting. This approach enables aggregate models to be flexible against demand fluctuations by varying production rate and production time horizons. Using subcontractors may associate companies with the outside partners in satisfying customer demand (e.g., Liang 2007).

- Accumulating seasonal inventories. By this approach, aggregate production planning models are developed by considering inventory capacities. Extra production in one period is stored in inventory and used in later time horizons (e.g., Meybodi and Foote 1995).

- Planning back orders. This approach allows aggregate models to consider back orders during their production planning horizons (e.g., Buxey 2005).

Aggregate models consider different costs to optimize production. These cost items usually include: a) basic production costs such as material costs, direct labor costs, and overhead costs. It is customary to divide these costs into variable and fixed costs; b) cost items associated with changes in the production rate such as costs involved in hiring, training, and laying off personnel, as well as overtime compensations; and c) inventory related costs which are determined according to the quantity of raw materials, work in process and final products supplied in warehouses and buffers.

The primary goal of aggregate production planning models is to minimize the total cost or maximize the profit of production system. Aggregate planning problems consider resources characteristics such as number of machines, level of workforce, hiring and firing costs of workforces. However, they seldom examine causes and consequences of workforce capability variations due to human work-related health problems. They usually assign a fixed value to the employees' performance and do not account for employee health-related productivity and quality changes (e.g., Van Hop 2007).

\subsubsection{Lot Sizing}

As a simple definition, lot sizing problems involve in minimizing the total cost of a system while considering set up, production and inventory costs. Lot sizing was started from a single item un-capacitated problem. This problem was first discussed by Wagner and Whitinin (1958). According to this problem, operations research analysts apply mathematical models to minimize the total costs of production, set up and inventory expenses for one item over a multi-period 
horizon in the presence of deterministic demands (e.g., Pochet and Wolsey 1988; Brahimi et al. 2006; Neidigh and Harrison 2010). The capacitated lot sizing problems present the other type of lot sizing. In this type, machines can produce different items in one period of the time while their production rates are limited by known capacity levels (e.g., Kohlmorgen et al. 1999, Meyr 2000; Ozdamar and Bozyel 2000; Gopalakrishnan et al. 2001; Hung et al. 2003).

Recently, the multilevel lot sizing problem has become the focus in lot sizing models. Production planning in these models is investigated for the end product at the final level as well as for all components and sub-assemblies that are needed to produce that finished product (e.g., Barbarosoglu and Ozdamar 2000; Dellaert and Jeunet 2000; Hung and Chien 2000; Xie and Dong 2002; Tang 2004). Production at one level creates demand for components at a lower level (dependent demand). At the final level, production is determined by market demand (independent demand).

Lot sizing problems are not limited to the introduced models, partial lot sizing and combination of lot sizing and scheduling models are the other set of mathematical solutions generated in order to optimize the behavior of systems (e.g., Cattrysse et al. 1993 , Ferreira et al. 2009). All of the mentioned models aim to replicate system performance completely. They investigate machine characteristics such as production rate, setup time and breakdown patterns. However, they rarely consider the employees' health related characteristics while several health problems can reduce the productivity rate of employees from 5 to 30 percent (e.g., Lerner et al. 2003 , Goetzel et al. 2004, Bultmann et al. 2007; Iverson et al. 2010). This thesis aims to follow lot sizing problem approaches in formulating operation systems while human factor aspects, affecting production flow, are integrated.

\subsubsection{Misperception of HF Aspects in OMPO Models}

A review of OM studies shows that human and technology aspects have been defined as important examples of the resources that can play critical roles in establishing systems with the sustained long term performance (e.g., Wan et al. 2011). An operation system can be described as a social-technological system since it includes both equipment (technological) and human (social) subsystems (e.g., Neumann 2004). Therefore, a realistic optimized performance of the system will result by investigating both human and technology aspects. However, OM studies do not take into account both equally. when OMPO models include human beings, they usually 
use simplifying assumptions regarding human's operational characteristics preventing them to replicate the realistic consequences of human involvement role in optimizing the performance of operation systems. These assumptions usually include:

a) Employee performance is constant without tiredness or on-job productivity loss (e.g., Bard et al. 2007). OMPO studies usually consider the average performance of employees in evaluating operation systems (e.g., Freivalds and Niebel 2009). They assume that this average value is the same for all workers in the system and is not varied due to occupational health problems (Boudreau et al. 2003). OMPO models infrequently consider WIH risk factors and their impacts on an employee health status and his/her functions during working hours, while the absence of employees from work (absenteeism effect), on job-productivity reduction due to health problems (presenteeism effect), and human health-related errors identified as the major health-related performance consequences.

b) Employees are not a major factor. OMPO models are mostly focused on technological features (e.g., Berretta and Rodrigues 2004; Boysen et al. 2008) and system characteristics such as demand uncertainty in the system (e.g., Ahmed et al. 2003) and they usually omit HF entirely and have rarely considered causes and effects of work-related employee health-states (e.g., Neumann and Dul 2010).

The misperceptions of HF exclusively as a Human Resource Management (HRM) tool and/or health and safety issue are the two reasons for employing these simplified assumptions regarding human capabilities. These assumptions consequently limit OM studies to integrating HF aspects and their consequences on human well-being and performance into OMPO models. In the next section, these misperceptions are discussed briefly.

\section{Human Factors and Human Resource Management}

HF is different from Human Resource Management (HRM). The HRM domain tends to be focused on developing practices to select and fit employees in an operation system in order to provide a sustainable performance for companies (Dul and Neumann 2009; Katou and Budhwar 2012). In contrast, HF has been focused on adapting the Working Environment (WE) in order to fit employees. HF intends to improve the performance of humans and consequently system by investigating the WE elements that affect the employees' well-being. In other words, human health condition is a critical intermediary element in improving the performance of 
both employees and systems. Figure 2.4 demonstrates the general approach of HF and HRM in improving the performance of employees.

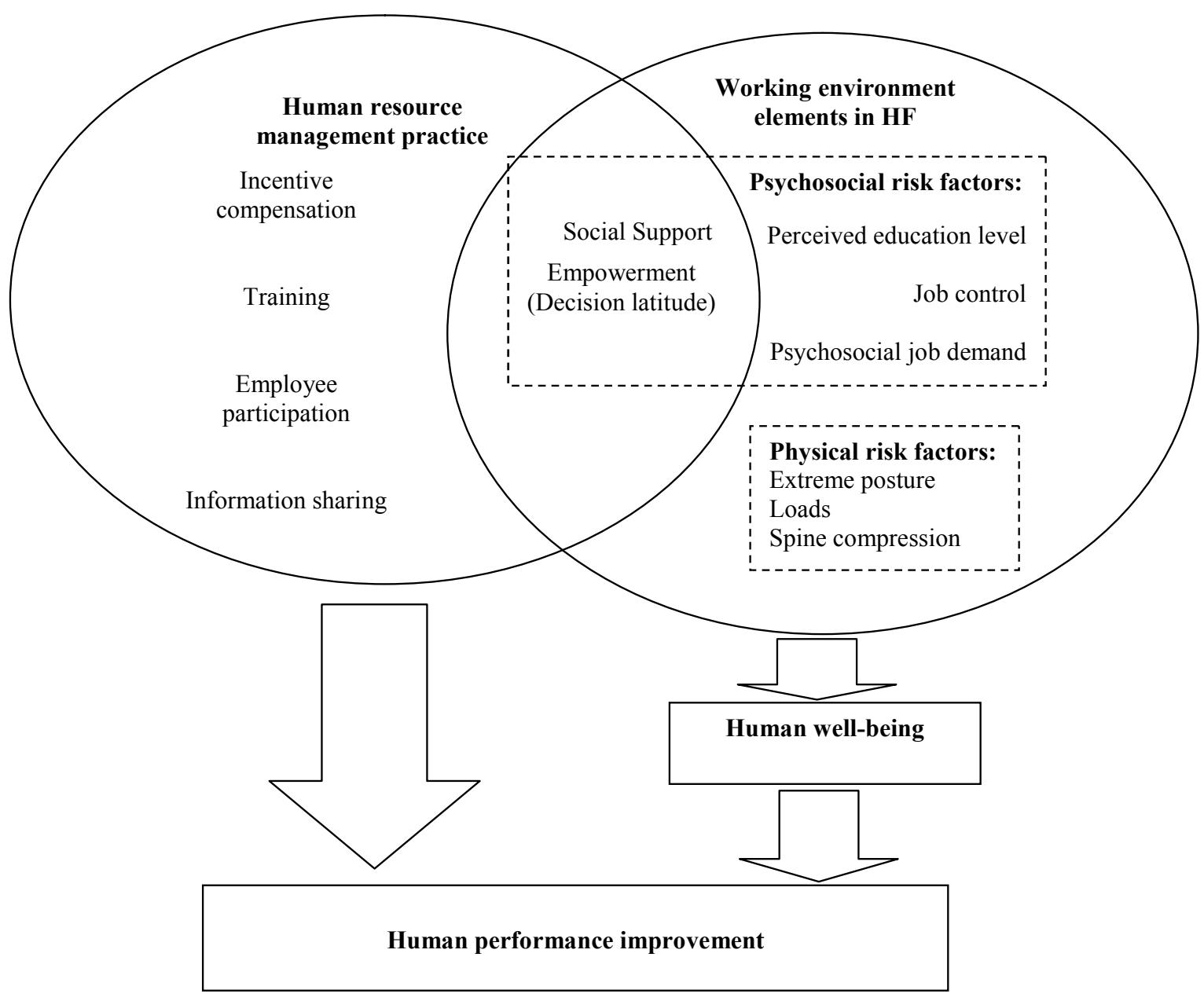

Figure 2.4: HF vs. HRM approach for human performance improvement

As it is shown, HF and HRM may have an overlap in considering the psychosocial elements of the WE affecting the performance of humans. For instance, human resource managers may advocate empowerment practices to pass considerable responsibility from operational management to individuals (e.g., Chenevert and Tremblaya 2009), in order to increase the employees' motivations and enhance their performance (Birdi et al. 2008). From the HF perspective, employee empowerment would be addressed as a psychosocial risk factor identified in terms of 
decision latitude aspects which have been linked to WIH (e.g., Bongers et al. 2002). Therefore, by improving psychosocial aspects of the WE, the employees have more responsibilities to make decisions in different working situations. Thus, work-related injury rates as well as associated OHS costs decline (e.g., Kerr et al. 2001).

$\mathrm{HF}$, therefore, is not just identified as a HRM tool. Instead, HF would be a complementary approach for HRM by evaluating both physical and psychosocial WIH risk factor effects on humans' well-being, and consequently their performance levels. Thus, bringing HF considerations to OMPO models will open a door for giving a better estimate of the performance of an operations system. It can also helps senior decision makers to understand how HF benefits should be realized.

\section{Human Factors and Health and Safety Issues}

Managers usually view HF as occupational health and safety issues to prevent injuries without providing return on investment (Jenkins and Rickards 2001). They often transfer the responsibility for employee well-being and safety of working conditions to health and safety department (Dul and Neumann 2009). However, the effects of WIH risk factors on human's performance (absenteeism and presenteeism effects) and direct and indirect costs of WIH, which are reported at 4-5 percent of the total gross domestic product (e.g., WHO 1999), have made it necessary to change this management view. In other words, HF is not only identified as a health and safety objective anymore and instead is a mean of supporting managers in improving the performance of human and reducing operation costs. HF can systematically link to OMPO models to better replicate a system behavior by considering the consequences of work-related employees' health problems at the system level evaluation.

\subsection{Summary of the Existing Research Gap}

Work-related ill health risk factors have negative consequences for employee health (e.g., Punnett and Wegman 2004, Rijn et al. 2010). Their effects on employee performance also create a substantial economic burden on organizations (e.g., Goggins et al. 2008). Human Resource and Skill Development Canada (HRSDC) reported over $\$ 18$ billion expenses annually due to occupational injuries ( 
lost-time injuries (Baldwin 2004; WSIB 2010a). However, OMPO models infrequently include the work-related human health consequences.

A review of OM studies shows that OM studies have been more focused on technological aspects such as machine breakdowns (e.g., Xia et al. 2012). They usually assign a fixed value to employee performance and do not investigate WIH risk factors and their effects on the employee's capability that influence the performance of systems. Although some studies discuss the positive effect of human resources in OM models (e.g., De Menezes et al. 2010), they are infrequently able to systematically integrate causes and effects of employee performance variation due to work-related health problems.

In contrast, the existing HF studies tend to be either exclusively focused on employee health, or limited to the instantaneous performance measures at the individual and workstation levels (e.g., Neumann and Dul 2010). Furthermore, unlike OMPO models, HF studies do not usually account for optimizing the behavior of the system including common system features such as buffer capacity and demand. In the following the important shortcomings of the recent HF studies are briefly noted:

- HF-related epidemiological studies have rarely investigated the consequences of WIH risk factor effects at the system and workstation level analysis.

- The existing ergo-economic models have generally focused on workstation level and, unlike most OM models fail to consider the entire system (e.g., Oxenburgh et al. 2004; Linhard 2005).

- The ergo-economic studies usually tend to use a snap-shot approach that looks into costs and benefits after and before an ergonomic intervention. They do not account for dynamically changing conditions of the operation systems and rarely apply OM methodologies (e.g., Lahiri et al. 2005).

This research aims to develop a new modeling approach to span this gap by including HF aspects when the performance of an operation system is optimized.

Chapter two reviewed the HF and OMPO research streams in order to better understand the existing gap between these two domains. This literature review demonstrates the necessity of providing a road map to investigate the effect of HF aspects by integrating the effect of HF into the system level optimization models while other features of operation systems and 
their dynamic behavior are included. This approach would be completed by developing a systematic methodology in order to enable OM mangers to evaluate causes and effects of human performance variation due to WIH at the individual, workstation and finally system levels. 


\section{Chapter 3}

\section{Research Methodology}

The research methodology used in this thesis is explained in this chapter and Operations Research (OR) tools used for system modeling and data analysis are introduced.

\subsection{Research Methodology}

This study pursues a hierarchical approach to combine the effects of HF aspects at the individual, workstation and system levels with other elements of the operation system when optimizing the performance of the system. This research approach involves : a) developing a generic modeling framework to integrate WIH risk factor effects into system performance optimization models. This framework aims to understand HF effects at the system level analysis through making rational connections among the individual, workstation and system level elements; b) determining the important work-related MSD and associated WIH risk profile from ergonomics literature; c) applying Markov chain and Logistic regression concepts to model employee health-states, taking into account the WIH risk factor effects (individual level); d) estimating workstation performance levels in terms of employee productivity level and quality defect rates (worksta-

tion level); and e) developing total cost optimization models for a manufacturing system taking into account HF effects (system level).

In order to simulate the real behavior of the operation system the following three steps were used to identify the parameters/factors, used in the modeling and data analysis:

- Reviewing HF and OR literature. 
- Justifying the relevant papers with respect to their research designs, samples of population, applied methods and the expertise judgment of their results.

- Designating focal parameters/factors from justified papers.

After this, sensitivity analysis approach has been considered within the range of the operating parameters in order to check the validity of the developed models. The results of this testing have been compared and assessed with respect to expert judgments in order to assess the models' robustness.

The methodology of this research is focused on employing empirical based results of previous HF studies and common statistical methods, used in epidemiology literature. This approach helps us replicate the effects of HF aspects, happened in a real working environment. Developing optimization models for a serial assembly system allows this research to test different scenarios for a specific case study. The serial assembly includes 4 workstations. There is a buffer between each two stations. This structure is widely used in OM literature as well as manufacturing environments (e.g., Mendoza and Venturab 2010; Iwasea and Ohnob 2011; Shang 2012). Each station has an operator who is accomplishing assembly tasks during the given time periods. This study models the chance of having work-related health problems taking into account WIH risk factor levels, exposed to each operator. A health-state Markov chain is generated for each employee to consider the dynamic effects of WIH risk factor changes. The assembly process starts from the first station. The demand during a given time period must be satisfied by the assembly line's throughput and/or subcontractors. The production rate of the station is affected by the operators' HF-related performance variation. The developed optimization models are numerically tested under different scenarios to assess the effect of HF design elements of the system on the optimum total cost of the operation system. For this research, the optimization procedure is completed by coding the associated integer programming with IBM-ILOG CPLEX v12.1. 


\subsection{OR Tools and Methods}

This research applies different OR tools and techniques in order to evaluate the impacts of HF integration into system performance optimization models. These methods are briefly introduced in the following:

\subsubsection{Logistic Regression}

Logistic regression is a generalized linear model used for binomial and multinomial regressions in order to predict the probability of occurrence of an event (Kleinbaum and Klein 2010). Like many forms of regression analysis, it uses several predictor variables that may be either numerical or categorical (e.g., Kleinbaum and Klein 2010). This method has been widely used in occupational epidemiological analysis to describe the relationship between a risk factor and human health outcomes (e.g., National Research Council 2001; Long et al. 2012). Generally, the output of the Logistic function estimates the probability occurrence of the health outcomes based on the predictor variables' exposure levels. There are two primary reasons for choosing the Logistic distribution. First, from a mathematical point of view, it is an extremely flexible and easy to use; and second, it can provide a clinically meaningful interpretation (Hosmer and Lemeshow 2000). In our research, Logistic regression will be used to estimate the health-state transition probabilities of Markov chain.

\subsubsection{Markov Chain}

Markov chain is a discrete stochastic process with the Markov property showing how a random variable modifies over the time (e.g., Ross 2003). The word "discrete" means that the stochastic process can take several discrete states and change its states randomly. The Markov property demonstrates that the conditional probability of the future states of the stochastic process depends only on the present state. The change of an state takes place with an associated transition probability. A given Markov chain enables the users to estimate the steady-state probability of each state. The steady-state shows how the system behaves over a long-period of time (e.g., Winston 2004 Pavitsos and Kyriakidis 2009). Markov modeling is a proved approach in analyzing human health-related issues (e.g., Kulasingam and Myers 2003; Cox and Popken 2004; Hatoum et al. 2005: Deuffic-Burban et al. 2007; Bowman et al. 2010) In this 
research, health-state change of a worker is modeled using a Markov chain for each employee.

\subsubsection{Linear programming}

A linear programming problem is defined as the problem of optimizing (maximizing or minimizing) a linear function subject to linear constraints (Winston 2004; Ferguson 2002). The constraints may be equalities or inequalities. Many practical problems in operations research can be expressed as linear programming problems. Moreover, a number of algorithms for other types of optimization problems work by solving linear programming problems as sub-problems (Ferguson 2002). Linear programming is heavily used in microeconomics and company management, such as planning, production, transportation, technology etc (Winston 2004). Therefore, many issues can be framed as linear programming problems (e.g., Tehrani Nejad Moghaddam and Michelot 2009). In this research, linear integer programming is used in optimizing the total cost model of the manufacturing system, with integer values for decision variables.

\subsubsection{Lattice Approach}

A Lattice approach is a discrete representation of a continuous process. Cox et al. (1979) proposed the binomial discrete time Lattice model by dividing a given time into a large number of small time intervals of length for a stochastic process. To describe the stochastic behavior of the demand, the Lattice approach has been extended for multiple geometric Brownian motions (e.g., Bollen 1999). The demand of a durable product is usually defined as uncertain and evolves with a stochastic product life cycle. Hence, a simple stochastic process is not always appropriate as demand undergoes fundamental changes when a product life cycle is described (e.g., the growth regime has a positive trend and the decaying regime has a negative trend). For this reason, a regime-switching process, which is a stochastic process that allows the parameters of the stochastic variable's distribution to take on different values in different regimes, is used to describe the demand of a durable product. We model the product life cycle using a regime-switching process in which different regimes of the product life cycle are described

by fundamentally geometric diffusion processes (e.g., Wahab and Lee 2011). In our approach, stochastic demand and life cycle of a durable product/service is modeled by a pentanomial Lattice approach. 


\subsubsection{Dynamic Programming}

The dynamic programming is a method of solving a multi-period (or sequential decision making) problem by decomposing the problem into many single-period problems (or simpler steps). In the case of stochastic demand within the product life cycle, a one period linear programming model that optimizes the performance of the system is solved. The optimization model consider all related costs and constraints. Then, by folding back to the previous time-step, the backward dynamic programing is applied to estimate the present value the optimum performance of the system at the beginning of the life cycle. Because of the characteristics of the proposed research, the most appropriate methodology is stochastic dynamic programming to minimize the total expected cost at the beginning of the planning period (e.g., Wahab and Lee 2011). 



\section{Chapter 4}

\section{Integration of human factors aspects into operations management performance optimization models: A modeling framework}

In order to span the gap between Human Factors (HF) and Operations Management (OM) research streams, this chapter introduces a framework integrating HF aspects into Operations Management Performance Optimization (OMPO) models. The novelty of the framework is based on prioritizing employee health status as an intermediary element in analyzing the behavior of a system's performance. This chapter provides a road map for evaluating the impact of work-related risk factors including physical and psychosocial aspects and their consequent human effects on optimizing the performance of operation systems.

The developed framework proposes a systematic approach to extend existing OMPO models by combining HF effects with the other features of an operations system such as customer demand, inventory and production costs. This framework can help managers understand the economic consequences of improving HF aspects at the system level. 


\subsection{Modeling Approach}

The overall modeling framework is based on a hierarchy of sub-models, illustrated by Figure (4.1). These three sub-models are identified as:

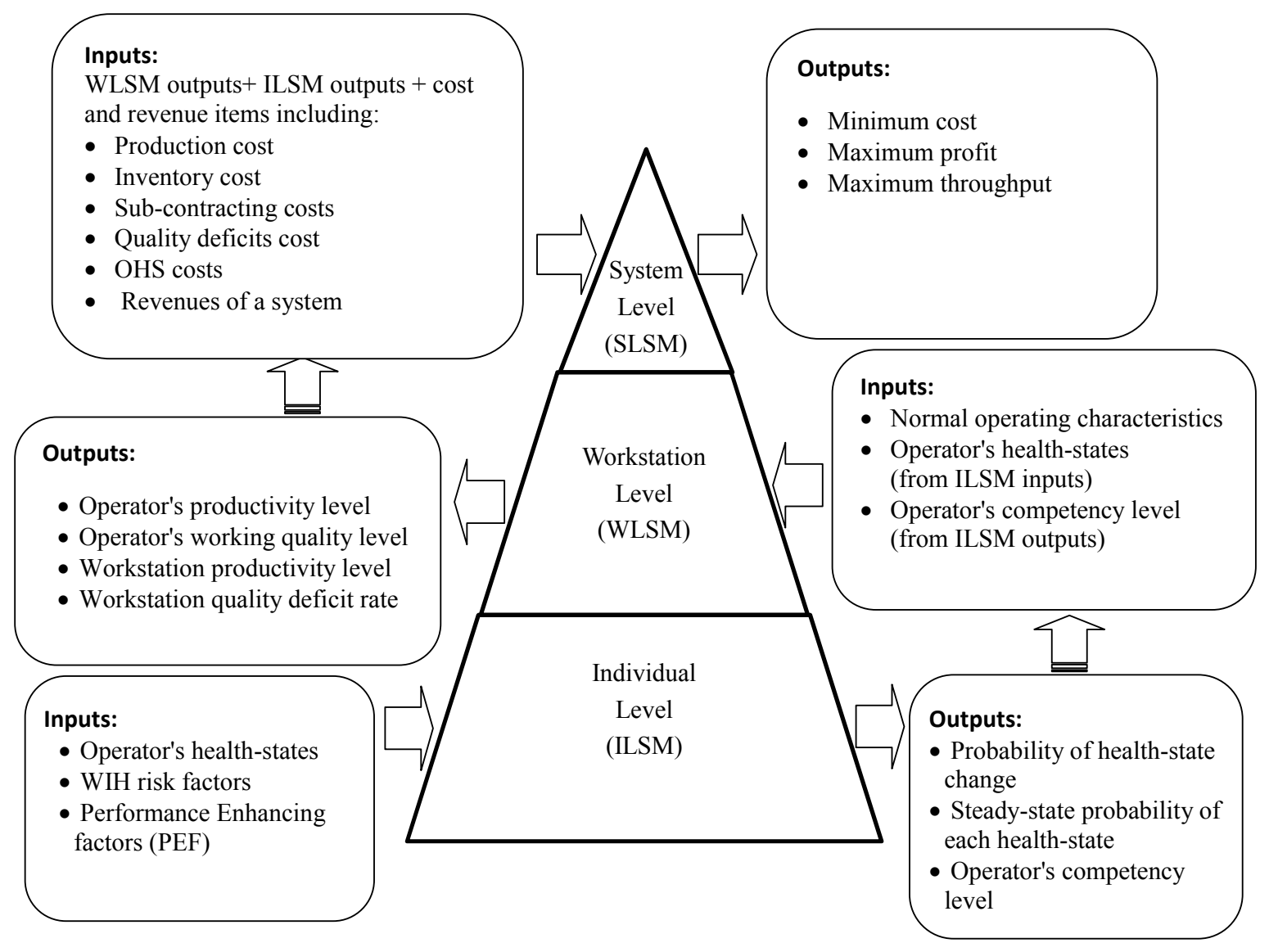

Figure 4.1: Developed modeling approach

a) The Individual Level Sub-Model (ILSM). At this level, the effects of Work-related Ill Health (WIH) risk factors on human health-states are considered. These health-states are defined with respect to different health conditions such as being healthy, having pain at work and become absent due to work-related injury.

b) The Workstation Level Sub-Model (WLSM). At this level, employee health-related productivity and quality deficits are considered with respect to the individual level outputs.

c) The System Level Sub-Model (SLSM). At the system level, the overall performance is opti- 
mized for a given system configuration and individual risk profile. OM techniques can be used to optimize the system's performance while considering the effects of HF involved from the ILSM and the WLSM. The details of each sub-model are described in the following sections.

\subsubsection{Individual Level Sub-Model (ILSM)}

The aim of the ILSM is to model an individual's response to both health risks and Performance Enhancing Factors (PEF) in the workplace. WIH risk factors are important elements affecting an employee's performance via the changing of their health conditions (e.g., Kerr et al. 2001). The ILSM model would be able to assess the consequences of exposure to these risk factors on the probability of change in the employee's health-state. The relationships between WIH risk elements and human health conditions are determined from epidemiological studies of WIH estimation (e.g., Ramond et al. 2010, Punnett and Wegman 2004, Devereux et al. 2002)

As demonstrated by Figure 4.2, the individual's initial health-states must be identified and set as the initial inputs of the ILSM. These health-states are defined according to HF and epidemiological tools investigating employee work-related health (e.g., Dawson et al.2009). For example, an individual's health-state can be, here, scaled from 0 to 4 ; where $0=$ healthy, $1=$ some pain (stay at work), $2=$ minor injury (stay at work), $3=$ injury (temporarily absent), and 4 = permanent disability. This scale can be adapted according to the needs of the model's users to include all possible employee health outcomes. Additional inputs to this sub-model can include the exposure of physical and psychosocial WIH risk factors, which are contributing to changing the employees' health-states.

For example, peak biomechanical loads, repetitive movements, job satisfaction and lack of job control are identified as the physical and psychosocial risk factors which had the main contributions in developing work-related low back pain for operators working in an automotive company in Canada (e.g., Kerr et al. 2001). To quantitatively determine the relationship between a WIH risk factor and an operator's health-states, statistical techniques, which are applied in epidemiological analysis such as such as cox regression, partial least squares and survival analysis, can be used in the ILSM. These techniques enable the framework's users to estimate the contribution of each WIH risk factor (independent variable) in developing the defined health-states (dependent variables). This type of quantification enables the modeling approach to estimate the probability of changing health-states, taking into account WIH risk 


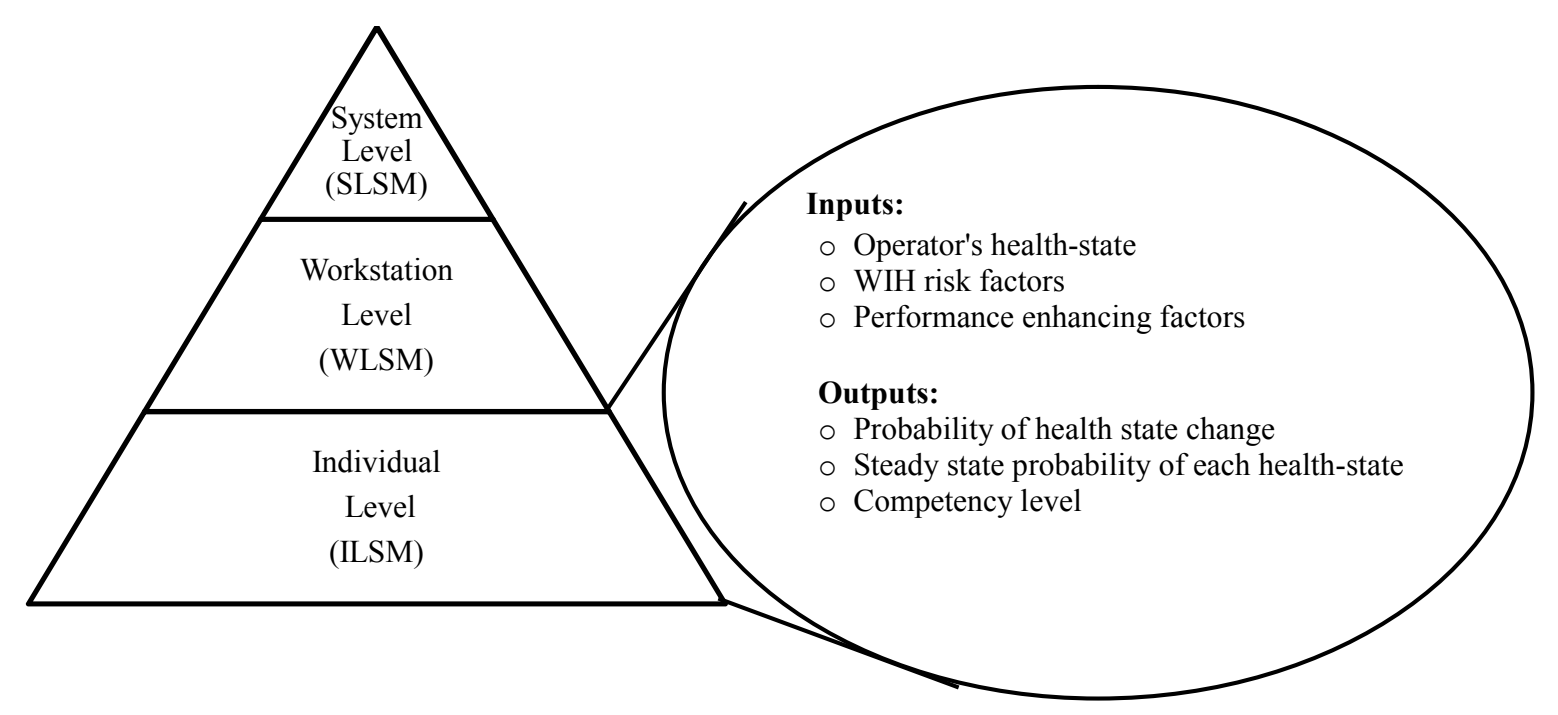

Figure 4.2: Individual Level Sub-Model (ILSM)

factor exposure levels. In general, as the exposure of a WIH risk factor increases, the probability of a negative shift in the health-state over a time period increases. Survival analysis is an example of statistical methods that can be applied to estimate the probability of health-state change with respect to the determined relationships between work-related health-states and WIH risk factors (e.g., Marcus et al. 2002).

Due to the dynamic nature of the Working Environment (WE), the operator's health-state will change periodically in response to different effects of work-related risk factors. Subsequently, the steady-state probability of being in each health-state, for example, the probability of being in a "minor injury" state (but staying at work) in the long run, can be calculated by integrating statistical and mathematical stochastic modeling into the ILSM. In each health-state, the operator works with a different performance level. Hence, the steady-state probability of each health-state is needed at the system level analysis to integrate the performance variation of humans into system performance optimization models.

Other than work-related health conditions, PEF such as learning and competency gains affect the operator's performance (e.g., Jaber et al. 2009), which can be included in the ILSM. The competency level of each employee at each workstation in the given time-periods can be estimated as a function of the "standard" time for the given task, where full competency is 
attributed as $100 \%$ corresponding to the normal performance of a normal employee. Standard learning functions (e.g., Jaber and Guiffrida 2007) can be applied to the ILSM in order to follow the employee's competency improvement that accrues as the individual learns to perform a task better over the time. The competency of a new employee, for example, might be set to $25 \%$ if the new employee is expected to take four times longer than normal to complete a task on his/her very first try. The employees' competency levels and the calculated probabilities of the employees' health-states are passed along to the next stages of the modeling framework in order to complete the workstation and system performance analysis.

\subsubsection{Workstation Level Sub-Model (WLSM)}

The aim of the WLSM is to determine the impact of the employees' health-states and competency levels on the overall performance of their workstations in terms of productivity and quality measures.

In the first step, as shown by Figure (4.3), the technical operating characteristics such as the planned production rate of each workstation are defined under "normal" conditions. These features will be based on the technical design of the workstation, which can be quantified through the use of a predetermined technique, such as Methods-Time Measurement (MTM). For instance, a given workstation produces twenty products every hour when staffed by a healthy and trained (fully competent) employee. By knowing the performance index of the workstation in the normal conditions, the WSLSM helps the framework's users specify the current performance of the workstation in comparison with its normal conditions.

In the second step, the overall productivity level of an operator can be calculated based on the defined health-states and his/her current competency level during a given time period. The WLSM can employ validated measurement tools such as the Work Limitation Questionnaire (WLQ) to quantify the impact of the current health-states of operators on their work activities (e.g., Tang et al. 2013). Subsequently, the production rate of each workstation is determined according to the health and competency related productivity level of the associated employees. For instance, by determining the relation between the health and competency related performance reduction of an operator and the production rate of his/her workstation, the WLSM estimates that an in-pain operator at work with $100 \%$ competency level decreases the production rate of the corresponding workstation station by $10 \%$. 


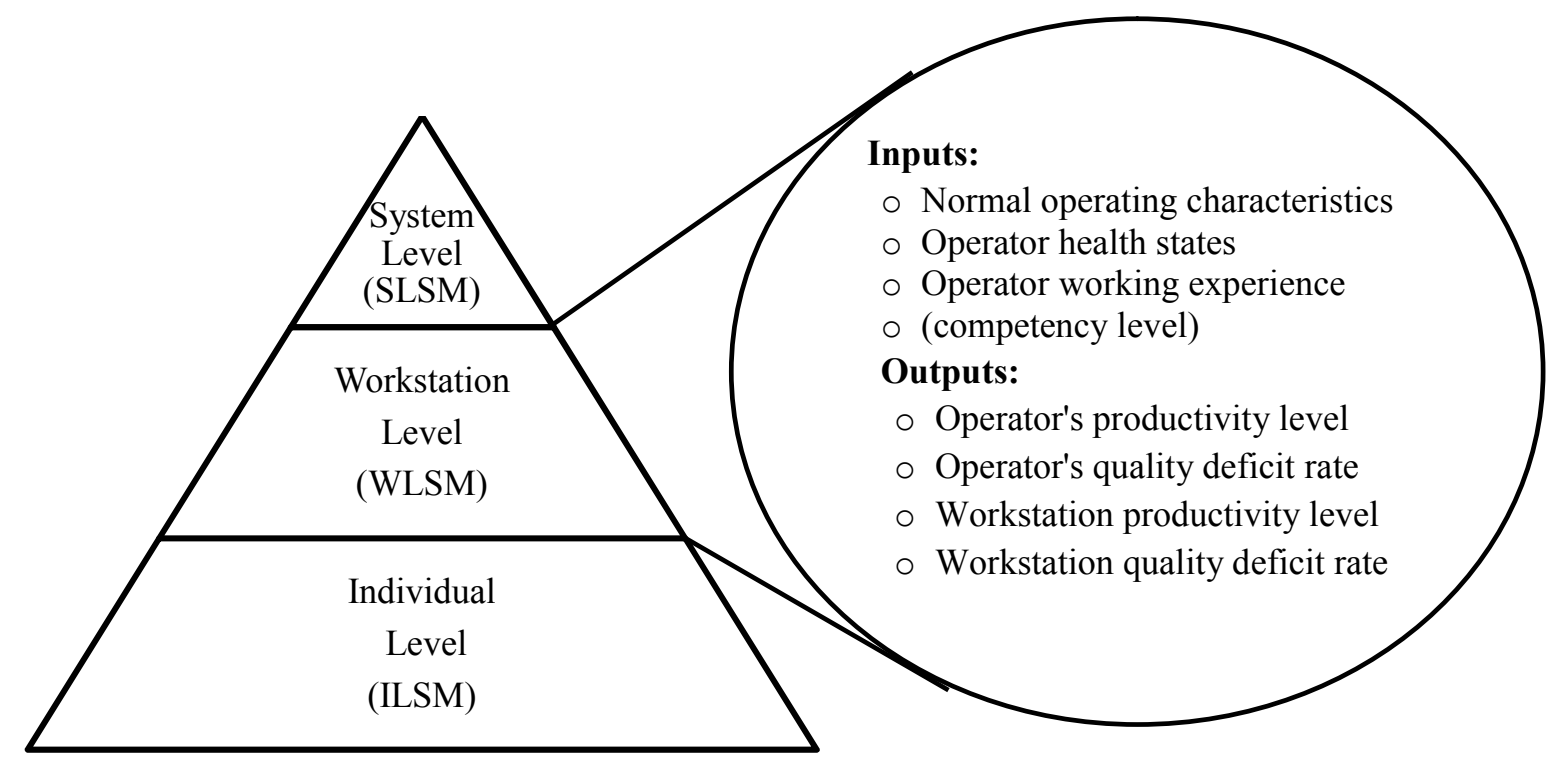

Figure 4.3: Workstation Level Sub-Model (WLSM)

Similarly, the impact of the operator's health-state and competency level on his/her error rate resulting quality deficits can be calculated for each station. Generally, an operator with poorer health or a lower competency can be expected to make more errors leading to increased quality deficits compared to a healthy, well trained worker. There is a growing awareness and empirical evidences for the relations between WIH risk factors and quality deficits in HF literature that can provide qualified relationships to support this sub-model (e.g., Erdinc and

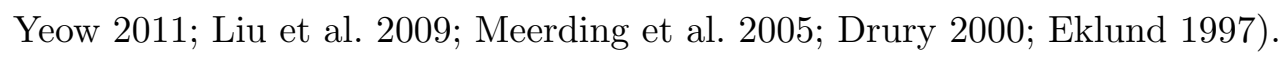

As demonstrated by Figure (4.3), by applying the available empirical relations, the WLSM can reveal the interactions between the technical design aspects of workstations, the operators' health-states and competency levels, and the resulting productivity and quality performance. These productivity and quality levels from each workstation are the outputs of the WLSM and are passed along to the next conceptual stage, the system level sub-model.

\subsubsection{System Level Sub-Model (SLSM)}

The aim of the SLSM is to optimize the overall performance of a system based on: the operators' work-related competency and health effects, imported from the ILSM, the identified 
performance of each station determined by the WLSM, the interactions among workstations as defined by the design of the system (such as a serial flow line for example) and financial items, used for optimization. The optimization model in the SLSM can focus on financial performance of the system, in terms of maximizing profit, throughput, or minimizing total cost. Figure (4.4) shows the details of inputs and outputs of the SLSM. For example, the total cost of a system can be attributed to the objective function of the optimization model. This function includes the cost items involved in producing final product such as production cost, OHS costs, insurance costs, inventory cost. The objective function is also constrained by the operating characteristics of the system.

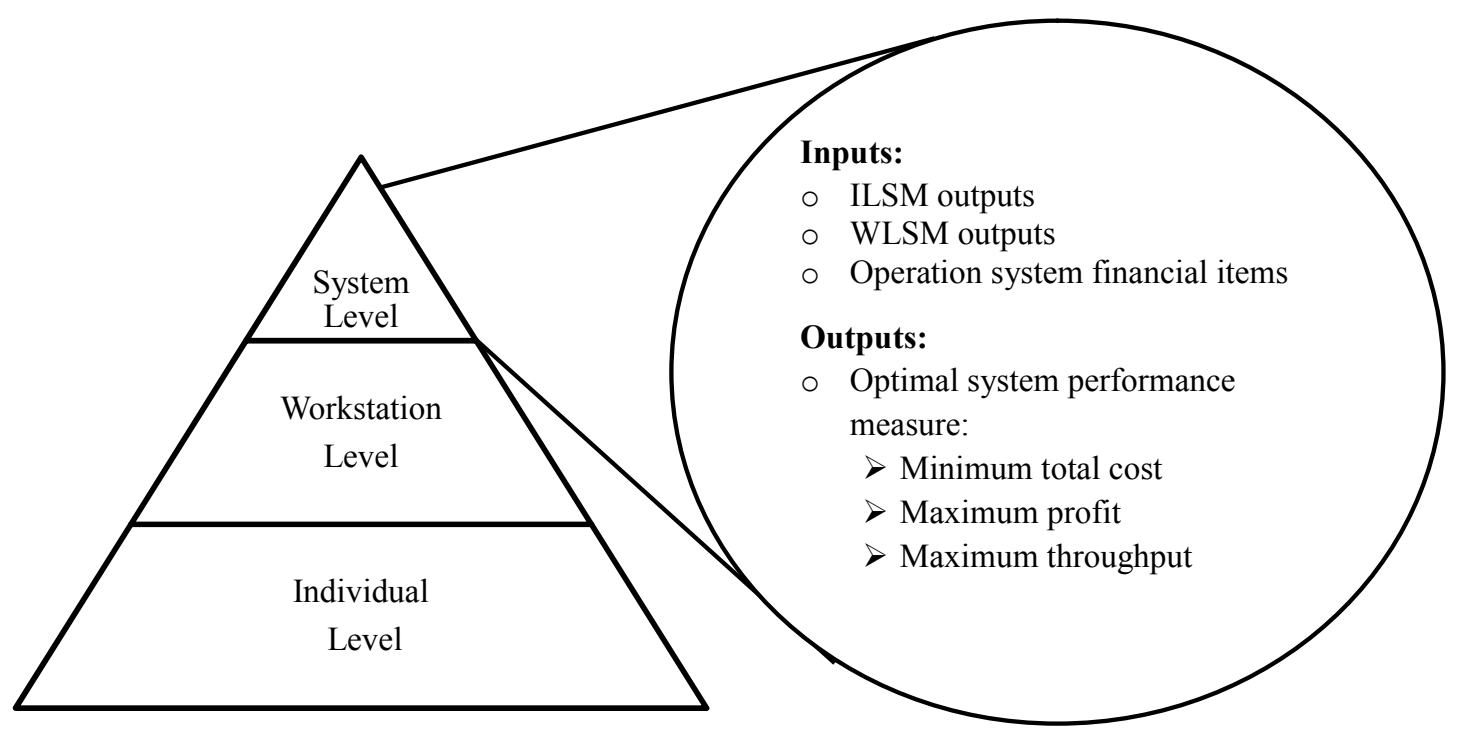

Figure 4.4: System Level Sub-Model (SLSM)

From the ILSM's outputs, the steady-state probabilities of the operators' health-states can be incorporated into the optimization model to consider work-related health effects. These probabilities help the framework's users examine the impacts of WIH risk factors on the overall performance of the system by creating the expected values for the system's variables which are varying as the operators' health-state change. Depending on the health-related productivity and quality levels, the SLSM also assists the users to estimate the expected total cost of operation, taking into the account the operators' work-related performance variations.

As discussed in the WLSM, the operators' performance variation is determined according 
to their defined health-states and competency levels. Changes in WIH risk factor levels vary the probability of being in each health-state, causing different productivity and quality levels for the operators. Consequently, the expected production rate and the production cost of the system are changed due to WIH risk factor variations.

Other than employee performance variation, an operation system includes several operational constraints affecting the performance of the system. These constraints are considered in the SLSM when a performance optimization model is developed. The examples of the mentioned constraints include: a) interactions among workstations; b) the capacity of workstations, c) the technological performance limitations of the system d) the total budget available for a given period to operate the system, e) the number of working shifts per day.

To determine the optimum performance at the system level, the SLSM requires financial information such as: the price of end products, the penalty cost of unmet demand, the production cost, the outsourcing expenses (subcontracting cost). This financial information can be employed by the developed optimization model at the SLSM. The possible cost items associated with the optimization model are briefly explained in the following.

Production cost is one of the important inputs at the system level of analysis. Economists and operation managers typically define the production cost as the combined costs of the factors of production incurred in producing goods or services such as labor, maintenance, raw materials and capital. Inventory cost is the cost item which is constrained based on the inventory level in the given period of time and demand uncertainties.

Third party purchasing and sub-contracting costs are the other operation system cost elements considered by the SLSM. Depending on the production policy, these costs can show the HF-related productivity changes at the system level, as sub-contracting are used to overcome WIH related production losses. For instance, due to poor HF design of the workplace, an operator may enter to the pain state while continuing to work. Hence, the corresponding workstation's production rate declines due to the operator's health-related productivity and quality losses. This reduction causes the whole system productivity level to be reduced as well. Consequently, the company cannot satisfy the demand on time and is forced to purchase the final products from outside (subcontractors) to satisfy the customer demand.

The value of defective products is another indicator in the total cost model that is subject to HF effects (e.g., Yeow and Sen 2003). For instance, if an operator is working in the pain-state 
and needs to bend his/her body to take an accurate dimensional measurement of the workpiece, then, there could be an increased probability of the individual making a measurement error and of a quality deficit in the resulting product. Similarly, due to a new replacement operator's lower competency level there is an increased probability of errors, quality defects, or reduced throughput (e.g., Jaber and Sikstrom 2004). These types of human-related working quality deficits incur expenses to rework or scrap defective items. Determining the relationship between the operators' health-states and quality deficits can be based on field observation, scientific data, company records and/or managerial experience.

To the author's knowledge, the developed modeling framework is unique in facilitating the consideration of occupational health and safety (OHS) cost items in the SLSM. While OHS costs must be adapted to each specific context, the examples of these cost items include: medical and rehabilitation expenses, payroll taxes and fringe benefits, acquisition cost, hiring and administration cost, training costs, and premium insurance cost (e.g., Oxenburgh et al. 2004, Lahiri et al. 2005). OHS-related expenses include both direct and indirect costs. The premium paid by a company to buy the insurance is also frequently categorized as an indirect OHS cost which usually depends on the type of the industry and the company's OHS record. Indirect costs are estimated between 1 to 10 times of direct costs (e.g. Leigh et al. 2004). By using OM techniques such as integer programming in the SLSM, it becomes possible to optimize the total cost of the system while the impacts of the modeled HF effects which are normally hidden from decision makers using conventional models are integrated. This integration enables the modeling framework's users to consider economic consequences of HF, related to the ergonomic design of the workplace, in analyzing the performance of the system. 


\subsection{Illustrative Example}

In this section, we use the case of a simple serial flow production system to illustrate how the modeling approach operates in a realistic situation. To model WIH risk factor effects, the author uses 4D Watbak software analyzing the operator's posture.

\subsubsection{Model Development}

In the serial system, product assembly starts from the first station. The operator of this station carries out initial assembly tasks. Then, the incomplete product will be transferred to the next station. This process continues until the product assembly is completed. The final product will fulfill customer demand. The production of the serial system is directly affected by the performance of its workstations. Operations research studies often consider this direct effect in terms of bottle neck problems ( e.g., Betterton and Silver 2012). Hence, any disruption in the performance of workstations can negatively affect the throughput of the sample assembly line. The performance of each workstation, in this example, is assumed to be dominated by the associated operator's operation characteristics. This illustrative example focuses on the healthrelated performance variations of employees and their subsequent impacts on the operation of the serial system. The design of the assembly system specifies working-load levels, working task repetition levels and the operators' working postures. HF analyst evaluate these elements to determine the effect of WIH risk factor on an operator's health condition (e.g., Marcus et al. 2002 ).

To consider work-related health effects, it is assumed that the operators of the assembly line have a probability of having work-related Low Back Pain (LBP), as a common occupational health problem in manufacturing environment. The health-state of each operator can be defined as: a) healthy, when the operator is healthy without any LBP symptoms; b) pain at work, when the operator is injured but stays at work with work-related low back problem; and c) absent, when the LBP forces the injured operator to be absent from work. It is assumed that a new employee is replaced. As mentioned in the ILSM and WLSM, medical and HF measurement tools enable us to measure the operators' health conditions and their performance levels at each defined health-state.

At the Individual level, the ILSM will determine the steady-state probability of being in each 
health-state. This probability depends on the magnitude of WIH risk factor exposure levels and the duration that an operator is exposed to the risk factors. While many different WIH physical and psychosocial risk factors exist, this example will focus on cumulative compression loading, as one of the common and important physical risk factors of the work-related LBP (e.g., Kerr et al. 2001). The cumulative compression load can be understood as the integrated of instantaneous loads on L4/L5 joints of the spine of the employees over a shift. It is scaled based on Mega Newton seconds per shift. The value of the cumulative compression level can be changed according to working load and number of assembly jobs (repetitions) in a shift. To estimate this value, Watbak has been used as a bio-mechanical modeling tool, calculating acute and cumulative loads at the body joints (Neumann et al. 1999). This modeling tool works on the basis of employee posture analysis taking into account working tasks' durations and repetitions. As a standard ergonomic practice, the poorest ergonomics-related employee's posture is used in Watbak to determine LBP risk levels. Figure (4.5) shows an example posture of an operator used in this section to estimate the operators' cumulative compression levels in terms of changing working load by demand.

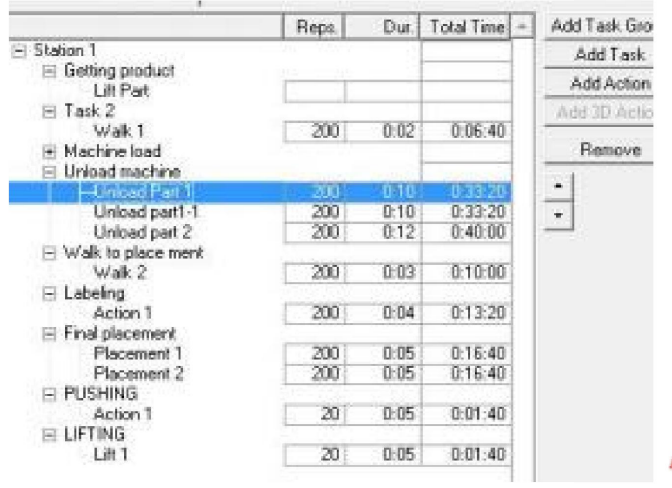

Cumulative compression exposure: $27 \mathrm{MNs} / \mathrm{shift}$ Demand is equal to 4400 units per month (200 items per shift)

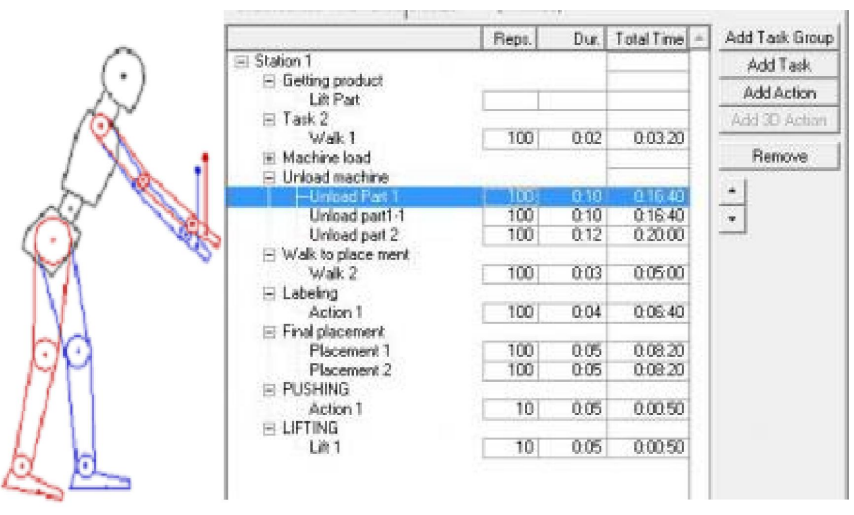

Cumulative compression exposure: $20.8 \mathrm{MNs} / \mathrm{shift}$ Demand is equal to 2200 units per month (100 items per shift)

Figure 4.5: An operator posture analysis with different task repetitions

At the workstation level, WLSM enables the users to estimate the performance of the assembly line's operators in terms of their productivity and quality levels. It is assumed that the healthy worker is $100 \%$ productive with negligible errors. However, when an operator is in the pain-state, the presenteeism effect results in decreasing the productivity and quality levels 
due to work-related LBP (Escorpizo 2008, Allen et al. 2005). In the absent state, a lower competency level of the replacement worker decreases the production of the corresponding workstation in the assembly line (e.g., Jaber and Khan 2010).

At the system level, the SLSM enables the users to optimize the total cost of the assembly line. The example model uses the probability of being in each work-related health-state to determine the expected cost of production, varied according to the operators' health-related performance variations. The optimization model can be constrained by the operational features of the system, discussed in the SLSM section.

\subsubsection{System Modeling by Integrating HF Effects}

Without including HF aspects, the developed optimization model would be unable to account for the operators' work-related health effects. However, in reality, working conditions and the HF design of the workplace change WIH risk factor exposure levels, incur negative financial effects (e.g., Goggins et al. 2008). To track these effects at the system level performance optimization, the dynamics involved in the sampled assembly system is described in terms of demand change.

In general, the assembly system faces demand fluctuation during the given time periods due to uncertain market condition. This type of fluctuation varies the production of the system to meet the demand. As demand increases, working tasks' repetitions increase resulting in a higher cumulative compression loading. Working with this higher loading increases the injury risk.

For this example, with 2,200 units per month as an initial demand, the posture analysis with Watbak, shown by Figure (4.5), determines the cumulative load level as $20.8 \mathrm{MNs} / \mathrm{shift}$. By knowing this exposure level, the ILSM lets the framework users estimate the probability of the healthy, pain and absent states. They can be assumed to be $0.54,0.36$ and 0.10 , respectively. These assumed probability values are aligned with the chance of LBP reported on this job $(46 \%)$ which is determined by Watbak for the given posture. Watbak software uses its own database including empirical based epidemiology data to roughly estimate the probability of LBP reporting. Recall that, the developed modeling approach in this study aims to analytically determine the health-state probabilities by applying statistical modeling methods, introduced in the ILSM.

By facing demand increment, operation mangers may use over-time to increase the produc- 
tion level of the assembly line in order to satisfy the demand. These extra working hours may change working conditions due to increasing the number of assembly tasks (repetitions) causing to reduce recovery time and intensify working loads. Hence, the cumulative compression loading of the operators' increases which is consequently raising the chance of being injured. For example, due to increasing the demand level to 4,400 per month for the sampled assembly system, the cumulative compression level is raised by 30\% (27 MNs/shift). The ILSM employs this cumulative loading increment to estimate the new probability of the health, pain and absent states. The new probability values can be $0.40,0.45$ and 0.15 for the healthy, pain and absent states, respectively (these assumed new probability values are aligned with the Watbak LBP analysis, estimating $60 \%$ chance of LBP reporting).

The increased probability of the pain and absent states indicates that the operators spend more times in those health-states. Therefore, the average (expected) performance of the workstations is reduced due to the higher work-related productivity and quality loss of the "in-pain" and "replacement" operators (determined in the WLSM). This negative performance consequently decreases the production quantity of the workstations. Thus, although the over-time working may initially increase the production of workstations, it is compromised by decreased quality and increased production quantity losses due to work-related LBP of the operators.

Ultimately, the performance reduction of the workstations will interrupt the operational balance of the serial system. According to the HF literature, the employee health-related performance loss was estimated between $3 \%$ to $34 \%$. Therefore, this interruption may have significant effects on the total cost optimization model. In general, due to increasing working loads, the total cost of the assembly would be optimized at a higher level during the given time periods due to the negative effects of WIH risk factors on:

a) the throughput of the assembly system. The work-related productivity loss of the "inpain" operator and replacement worker limit the assembly's production volume. Therefore, more products are ordered to subcontractors, which are increasing third party costs.

b) the quality deficit rate of the assembly line. The work-related operators' error rates raise the number of scrap and rework products, which are increasing defective expenses. Usually, the defective costs are at least 2 times of the production cost.

c) OHS costs. Due to the higher chance of being injured, the expected medical and rehabilitation expenses increase. Hence, more claims are registered to the insurance companies. 
Since, insurance premium rate has direct relationship with the number of claims. the average insurance premium costs increase as well.

Without including HF aspects, the optimization model is unable to trace the negative impact of increasing the cumulative exposure level on the minimum total cost of the assembly system. Therefore, the performance of the assembly system is sub-optimized by underestimating the minimum total cost. Furthermore, the managers cannot predict the production loss due to the employee health-relate productivity and quality losses. From the other side, the proposed modeling framework enables the users to investigate HF effects while it also includes traditional features of operation systems such as inventory, production flow etc.

\subsection{Discussion}

This chapter proposes a framework integrating HF effects into OMPO models to study system performance, resource management and operating options. OMPO and HF literatures appear to be two isolated streams of investigation (e.g., Dul and Neumann 2009). On one hand, most studies in OMPO investigated system performance without taking into account the work-related human performance variations in an operation system (e.g., Ahmed and Sahinidis 2008). They usually use variables such as technological features of the system and product variety in order to optimize the system's output (e.g., Boysen et al. 2008). On the other hand, HF studies have primarily focused on occupational health aspects. To the author's knowledge, HF research field infrequently applies OM methodologies in a systematic approach. HF studies are usually supported by perceptions that attention to HF evaluation will only prevent compensation costs and not contribute to business goals or the system output, despite clear evidence to the contrary (Boudreau et al. 2003, Goggins et al. 2008, Dul et al. 2012). Therefore, there is a gap between OMPO and HF fields that poses a research opportunity to generate an integrated understanding of how these disciplines can together contribute to the design of operations and enrich human resource management. Such a methodology presented in this chapter has potential to help companies avoid the hidden costs of poor HF in the workplace, and find system designs that help them capitalize on their people's competencies in a sustainable way. 


\subsubsection{Benefits of Applying the Framework}

From operations management perspective, this conceptual framework poses a systematic approach to understand the performance of a system while including HF in combination with other elements of the system. This approach can be vital when humans are critically involved in operation. OMPO models attempt to calculate financial performance, but infrequently include all relevant human factor aspects. Hence, they are likely to underestimate the financial performance of an operation system. These expected but never realized returns on system investments have, in the context of missing attention to HF aspects, been dubbed "Phantom Profits" (Neumann 2004).

Flexibility is the ability of a company to respond to the demands from its dynamic competitive environment (Bhattacharya et al. 2005). One of the advantages of the proposed framework is to create an opportunity to explore options for a system to be more flexible, in order to cope with uncertainty in ways that avoid employee WIH and associated long term negative consequences. Such a model can help companies deal more reliably with uncertain variables such as customer demand. Companies should be flexible to be able to respond to the demand and perform effectively and efficiently in order to satisfy customers and meet deadlines (Slack 2005). The integration of HF aspects into OMPO models can help the production managers by bringing more options in a) matching the production rate with demand fluctuations; b) improving productivity levels; and c) decreasing quality deficit rates.

Practitioners aim to control production variation using a number of different strategies. Enhancing workstation technology, changing system design by adding or reducing workstations and instituting over-time work policies are common strategies to adjust production value and quality performance. The proposed framework can support the rapid assessment of the above strategies and help managers develop a safe, effective, and efficient plan. Since the proposed framework can identify relationships between employees' health-states and competency levels with their productivity and quality levels, practitioners have better information to manage production variation and maintain a sustainable and healthy working environment.

While further testing is required, from ergonomics perspective, the integration of HF aspects into the proposed modeling framework can establish healthier working environments that lead employees to feel better while they are working and to increase their competences in supporting their companies' strategic goals (Dul and Neumann 2009). One important advantage of the 
framework over conventional ergo-economic tools would be the integration of HF aspects into OMPO models in order to examine productivity and quality at both workstation and system levels. Both direct and indirect OHS costs can be incorporated to optimize the performance of a system while other system elements such as demand and throughput are also included. Furthermore, while existing ergo-economic methods mostly use static "snap-shot" assessment models, the approach described here would allow a long term modeling of system dynamics. This dynamic assessment capability is not common in the HF literature. The proposed framework does not optimize the heath-state change itself, but optimizes the system behavior for a given pattern of health-state change based on the defined WIH risk factors. Therefore, the framework can be used to investigate how to enhance the system performance by improving work-related physical and psychosocial WIH risk factors.

\subsubsection{Framework Challenges}

The conceptual framework follows a new approach in order to evaluate the behavior of any operation systems while investigating $\mathrm{HF}$ aspects in sufficient details. Novel modeling approaches always face challenges and the proposed framework is not an exemption. The important challenges for the proposed framework are referred to as feasibility and validity.

The major feasibility challenge is the availability of the required in-data. The feasibility of obtaining data depends on the scope and context of the system to which the framework is applied. Many WIH risk factors have been discussed in the existing HF literature including both physical and psychosocial workplace factors and their impacts on disorders for various body parts. But, obtaining hazard risk data, such as cumulative loading (e.g. Kerr et al. 2001) requires knowledge and the use of human posture modeling software which may not be readily available in many firms. Similarly, financial and industrial field data such as production cost per product and number of products produced in each station also have to be collected for the workstation and system level analysis. Obtaining this data can be troublesome if the company is, for example, only collecting data in an aggregate form for what is needed. In response to these challenges, we propose a number of tactics including: examining available research data, drawing on available regional statistical data, drawing on companies records, making field measurements, and applying managerial and employee judgments on critical relationships.

With a modeling framework scope that spans the individual, workstation, and system levels 
simultaneously with multiple types of $\mathrm{HF}$ and operational variables, determining the model validity can be a challenge. Furthermore, in-data may differ between application contexts when the proposed framework is, in principle, applied to manufacturing, service and health sectors. Hence, cross-site validation is difficult since the framework users may ask for different variables to investigate. The preferred approach here would be quantitative validation by simulating the system's past behavior. The limit in this regard depends on the accessibility of matching historical performance data in the firm, which may be problematic for the same reasons as feasibility challenges. Another form of the validity check would be more qualitative based on engineers and managers judgments who have previous experiences with the system. They review the response of the model to sensitivity testing within the range of operating parameters which are determined in a real working environment.

This modeling framework provide a systematic road map to integrate HF into optimization model at the system level analysis. To numerically investigate the benefits of this type of modeling, the next chapters develop optimization models including HF financial and operational effects. 



\section{Chapter 5}

\section{An analytical modeling approach to integrating $\mathrm{HF}-$ related productivity loss into a serial system performance optimization models}

In previous chapters the research gap between Human Factors (HF) and Operations Management Performance Optimization (OMPO) models has been discussed in details. Chapter five elaborates an analytical model which is spanning the gap by including HF aspects in minimizing the total cost of a manufacturing system. The optimization model is proposed for a serial system, considering physical and psychosocial Work-related Ill Health (WIH) risk factor effects.

The aim of this model is to make a connection between Operations Management (OM) and HF research streams by considering causes and consequences of employees' work-related health and productivity variations at the system level performance optimization. A modeling approach is developed that, first, determines the relationship between known WIH risk factor measures and their subsequent effects on employee health conditions. Then, these health conditions are modeled using a 2-state Markov chain and integrated into an optimization model, which also accounts for WIH risk factor effects. Other features of the system such as customer demand, inventory capacity, and production costs are also included. The developed model is numerically tested to evaluate the effect of different work-related Musculoskeletal disorders (MSD) on the 
performance of the operation system. This analytical study opens a new window to understand economic consequences of WIH effects and to enhance system performance by investigating working conditions.

The remainder of the chapter is organized as the following. Section 5.1 introduces an assembly system and their operational elements. It also describes major steps in the modeling approach in order to integrate WIH risk factor effects into the optimization of the assembly system's performance. In section 5.2, the proposed model is numerically tested by considering work-related low back and shoulder WIH risk factors. The developed model is examined with different scenarios to comprehensively evaluate WIH risk factors and their impacts on the optimized financial and operational elements of the system. In section 5.3, the advantages and limitations of investigating WIH effects are discussed from managerial perspective.

\subsection{Model Development}

This section introduces an optimization model integrating WIH risk factor effects. From different manufacturing structures, let us consider a serial assembly with limited capacity buffers between workstations. This workstation arrangement is widely used in OM literature as well as manufacturing environment (e.g., Iwasea and Ohnob 2011). The overall structure of the serial assembly line has been explained in the chapter three. For the readers' convenience, it is briefly described again.

It is assumed that the sample assembly line includes 4 serial workstations and there is an operator for each of them to accomplish assigned assembly tasks. Product assembly starts from the first station. The operator of this station carries out initial assembly tasks. Then, the incomplete product will be transferred to a buffer between the first and second stations. The second station brings the product from the buffer in order to perform its assigned tasks. This process continues until the product assembly is completed in all 4 stations. The final product will be transferred from the fourth station into the inventory to fulfill demand. Production is during regular and over-time hours. The backlog is not allowed and if the throughput of the assembly line cannot fully satisfy customer demand, it is assumed that subcontractors will fulfill the unmet demand during each given time period. WIH risk factors affect the operators' performance and machine failure vary the performance of the assembly line. However, this 
chapter focuses on the effect of WIH risk factors when the performance of the assembly line is optimized. Therefore, it is assumed that machines used in the system work perfectly, and the production rates of the workstations depend on the performances of the operators.

An operator who is working in the assembly line has a probability of having work-related MSD for a given time period due to the negative effects of exposure to WIH risk factors (e.g., Punnett and Wegman 2004). These effects change the operators' health status. In order to replicate the health condition changes, happened in a real working environment, the operators' health-states are categorized taking into account work-related health evolutions, resulted from previous epidemiology and HF studies (e.g., Kerr et al. 2001).

Epidemiological studies use different measurement tools to evaluate the effect of WIH risk factors in developing MSD symptoms in different human body areas (e.g., Dawson et al. 2009). They usually divide their study participants into people with and without work-related pain (e.g., Sim et al. 2006). Furthermore, recent HF studies have considered the contribution of the work-related pain in human productivity loss (e.g., Campo and Darragh 2012). Therefore, for the purpose of this research, the health condition of the operators is categorized in two states. The operators would be either healthy without having any MSD or may have pain due to musculoskeletal problems. These work-related health states are characterized as 0 and 1 , where 0 indicates the "healthy" state and 1 indicates the "pain" state with productivity loss.

It is assumed that an operator is $100 \%$ productive in the healthy state. However, the operator in the pain state has a lower production rate because of a) the on-job productivity loss, when he/she has pain or injury and stays at work (presenteeism) or; b) the replacement of a new worker, who has less competence and skills than the injured absent operator during the given time periods. For instance, due to repetitive lifting of heavy parts from storage, the health-state of an operator who is responsible for installing parts in the assembly line may be changed from the healthy to the pain during a given time period. This pain causes the operator to work with a lower production rate or to be absent, and replaced with a new worker who also has a lower experience and skills level. The health-state change can take place because of physical and psychosocial risk factors. As the hazard risks increase, the probability of the health-state change increases for the affected operators.

According to the defined health-states, a 2-state Markov chain is generated for each operator to calculate the steady-state probability of being in the health-states. Then, the total cost 
model of the assembly line is minimized by considering the effect of the operators' health-states and corresponding WIH physical and psychosocial risk factors. The total cost function includes the expected production cost of all workstations with respect to the operators' health-states, Occupational Heath and Safety (OHS) costs, inventory cost and subcontracting cost. It is minimized over a year, 12 consecutive months. Demand is assumed to be known in each month, but it varies from one month to another.

For numerical analysis, the health-related information is extracted from previous HF studies (Kerr 1997; Sim et al. 2006). The information is adjusted taking into account the epidemiology research and statistical reports of workers' compensation boards of Canada (e.g., WSIB 2010a: HRSDC 2010). It is assumed that WIH risk factor levels do not change due to demand variations. Different scenarios are evaluated to examine the effect of work-related MSD in the developed optimization model.

\subsubsection{Major Steps in the Modeling Approach}

There are two major steps to integrate WIH risk factor effects into the optimization model. In the first step, the model attempts to make a connection between the exposure level of WIH risk factors and the 2-state Markov chain elements. For this reason, Logistic regression, which is widely used in epidemiological analysis, is applied to examine the influence of various known WIH factors on the dichotomous outcome of an operator's health-state. The output of the Logistic regression generates the associated probability occurrence of the operator's health-states, according to the values of risk factor measures (e.g., Hosmer and Lemeshow 2000). Consequently, these health-state change probabilities will be used to generate a 2-state transition probability matrix (e.g., Azzalini 1994).

In order to determine the probability of being in the given health-states in each station, Logistic regression and corresponding transition probability matrix are generated for each operator individually. Equation (5.1) shows the formula used by the Logistic function to calculate the marginal probability of entering to the pain state, $v_{p j t}$, for an operator working in station $j$ in time $t$. This probability is subjected to change when the exposure levels of the investigated risk factors change. The output of Equation 5.1 is employed to generate the corresponding transition probability matrix of the health-states. 


$$
v_{j t}^{p}=\frac{\exp \left[\sum_{y=1}^{n} \beta_{y j} X_{y j}+\alpha_{0 j}\right]}{1+\exp \left[\sum_{y=1}^{n} \beta_{y j} X_{y j}+\alpha_{0 j}\right]} .
$$

Since WIH information is usually collected from annual base studies, the monthly base transition probability matrix for each operator is generated by raising the corresponding annual base transition probability matrix to the power of " $\frac{1}{12}$ ", demonstrated by Equation 5.2 . Finally, the monthly base steady-state probability of being in health-states are calculated according to Equation (5.3), let

$$
\begin{gathered}
{\left[\begin{array}{cc}
1-v_{j t}^{p}\left(1-u_{j t}^{p}\right) & v_{j t}^{p}\left(1-u_{j t}^{p}\right) \\
\left(1-v_{j t}^{p}\right)\left(1-u_{j t}^{p}\right) & u_{j t}^{p}+v_{j t}^{p}\left(1-u_{j t}^{p}\right)
\end{array}\right]^{\frac{1}{12}}=\left[\begin{array}{cc}
\Omega_{j \tau} & 1-\Omega_{j \tau} \\
\Upsilon_{j \tau} & 1-\Upsilon_{j \tau}
\end{array}\right],} \\
\pi_{j \tau}^{h}=\frac{\Upsilon_{j \tau}}{1+\Upsilon_{j \tau}-\Omega_{j \tau}} \text { and } \pi_{j \tau}^{p}=\frac{1-\Omega_{j \tau}}{1+\Upsilon_{j \tau}-\Omega_{j \tau}}, \text { where } \pi_{j \tau}^{h}+\pi_{j \tau}^{p}=1 .
\end{gathered}
$$

In the second step, the total cost function of the assembly line is minimized with respect to the health-related probabilities. The developed optimization cost model is described as follows.

$$
\begin{aligned}
& \operatorname{Min} \sum_{j=1}^{4} \sum_{\tau=1}^{12} c_{j \tau}^{r} \pi_{j \tau}^{h} P_{j \tau}^{h r}+\sum_{j=1}^{4} \sum_{\tau=1}^{12} c_{j \tau}^{r} \pi_{j \tau}^{p} P_{j \tau}^{p r}+\sum_{j=1}^{4} \sum_{\tau=1}^{12} c_{j \tau}^{o} \pi_{j \tau}^{h} P_{j \tau}^{h o}+\sum_{j=1}^{4} \sum_{\tau=1}^{12} c_{j \tau}^{o} \pi_{j \tau}^{p} P_{j \tau}^{p o} \\
& +\sum_{j=1}^{4} \sum_{\tau=1}^{12} b_{j \tau} B_{j \tau}+\sum_{\tau=1}^{12} s_{\tau} S_{\tau}+\sum_{j=1}^{4} \sum_{\tau=1}^{12} w l_{j \tau}\left(\frac{\pi_{j \tau}^{h} P_{j \tau}^{h r}+\pi_{j \tau}^{h} P_{j \tau}^{h o}}{z_{j \tau}^{h}}+\frac{\pi_{j \tau}^{p} P_{j \tau}^{p r}+\pi_{j \tau}^{p} P_{j \tau}^{p o}}{z_{j \tau}^{p}}\right) \\
& -\sum_{j=1}^{4} \sum_{\tau=1}^{12} w l_{j \tau}\left(\frac{\pi_{j \tau}^{h} P_{j \tau}^{h r}+\pi_{j \tau}^{h} P_{j \tau}^{h o}+\pi_{j \tau}^{p} P_{j \tau}^{p r}+\pi_{j \tau}^{p} P_{j \tau}^{p o}}{z_{j \tau}^{h}}\right)+I N+I C
\end{aligned}
$$

subject to:

$$
\begin{gathered}
\pi_{j \tau}^{h} P_{j \tau}^{h r}+\pi_{j \tau}^{p} P_{j \tau}^{p r}+\pi_{j \tau}^{h} P_{j \tau}^{h o}+\pi_{j \tau}^{p} P_{j \tau}^{p o}+B_{j \tau}-B_{j \tau+1}-\pi_{j+1 \tau}^{h} P_{j+1 \tau}^{h r} \\
-\pi_{j+1 \tau}^{p} P_{j+1 \tau}^{p r}-\pi_{j+1 \tau}^{h} P_{j+1 \tau}^{h o}-\pi_{j+1 \tau}^{p} P_{j+1 \tau}^{p o} \geq 0 \quad j=\{1,2,3\} . \\
\pi_{4 \tau}^{h} P_{4 \tau}^{h r}+\pi_{4 \tau}^{p} P_{4 \tau}^{p r}+\pi_{4 \tau}^{h} P_{4 \tau}^{h o}+\pi_{4 \tau}^{p} P_{4 \tau}^{p o}+B_{4 \tau}+S_{\tau}-B_{4 \tau+1} \geq d_{\tau} .
\end{gathered}
$$




$$
\begin{gathered}
P_{j \tau}^{h r} \leq s h_{j \tau}^{r} m_{j \tau} z_{j \tau}^{h} . \\
P_{j \tau}^{p r} \leq s h_{j \tau}^{r} m_{j \tau} z_{j \tau}^{p} . \\
P_{j \tau}^{h o} \leq s h_{j \tau}^{o} m_{j \tau} z_{j \tau}^{h} . \\
P_{j \tau}^{p o} \leq s h_{j \tau}^{o} m_{j \tau} z_{j \tau}^{p} . \\
S_{\tau} \leq p c t_{\tau} d_{\tau} . \\
0 \leq B_{j \tau} \leq b m_{j \tau} . \\
P_{j \tau}^{h r}, P_{j \tau}^{p r}, P_{j \tau}^{h o}, P_{j \tau}^{p o}, S_{\tau}, \text { and } B_{j \tau} \text { are integers }
\end{gathered}
$$

The objective function, Equation (5.4), seeks to minimize total production, inventory, subcontracting and OHS costs. The production cost includes the expected production cost of all workstations, accumulated during 12 months. For each workstation, the expected production cost during period $\tau$ is determined by multiplying the unit production cost with the expected production quantity produced by the corresponding operator in regular hours and over-time working periods, according to his/her defined health-states. The inventory cost is incurred according to the value of buffer levels multiplied by the unit inventory cost of the product, accumulated over 12 months. The subcontracting cost is incurred whenever demand is not met by production during the given time periods. OHS costs are considered in terms of insurance premium cost, indirect costs and wage loss cost.

Constraints (5.5) and (5.6) guarantee the flow of products through the serial assembly line. Constraints (5.7), (5.8), (5.9), and (5.10) are subject to the available time-duration of being in each health-state during the given time periods. It is assumed that there are 22 working days in each month. Production rate in each health related-state is determined according to the literature in OM and HF studies (e.g., Martimo et al. 2009). 
Constraints (5.11) and 5.12 limit subcontracting and buffer capacity levels. The model allows the company to outsource production up to 40 percent of the demand in each time period. Maximum buffer capacity is 10 products per buffer.

From the company's point of view, insurance premium cost represents medical and compensation expenses spent due to work-related pain or injuries. For this study, the OHS performance sensitive approach is used to determine insurance premium rate with respect to medical treatment and compensation expenses (e.g., Riel and Imbeau 1998). Then, this rate is multiplied by the number of the employees to calculate the total insurance premium cost of the assembly system. Equation (5.13) shows the insurance premium cost, IN, adjusted in a way to consider WIH effects.

$$
I N=4 A I N \sum_{j=1}^{4} i n w_{j}\left(\sum_{j=1}^{4} \sum_{\tau=1}^{12} m e_{\tau} \pi_{j \tau}^{p} m_{j \tau}\right) .
$$

The unit cost of insurance, $A I N$, is defined as per dollar of medical treatment and compensation expenses credited per dollar of insured salaries. $i n w_{j}$ is the average of the annual insurable salary per worker. These parameters are estimated and adjusted according to the Workplace Safety and Insurance Board (WSIB) of Ontario, Canada, Ontario Ministry of Labor and Canada's statistics reports (e.g., HRSDC 2010; WSIB 2010a).

Indirect OHS costs include overheads such as personnel acquisition costs spent in the recruitment, hiring and administration of the replacement employees and training expenses spent to enhance injured and replacement employees' skills. There is, however, no specific method to calculate these indirect costs. Several studies have estimated these expenses in comparison with medical care and benefit costs (e.g., Leigh 2011). The results varied between 1 and 10 times of medical and benefit costs. The developed optimization model applies the WSIB estimation for indirect OHS cost effects in Equation (5.14), which is 3 times of the medical care and benefit costs.

$$
I C=3 \sum_{j=1}^{4} \sum_{\tau=1}^{12} m e_{\tau} \pi_{j \tau}^{p} m_{j \tau} .
$$

Wage loss which is determined by the objective function, is another important OHS cost considered in the developed model. As noted in Equation (5.4), this cost reflects the amount of payments for working hours which are lost due to the operators' health-related insufficient productivity during the given time periods. 


\subsection{Numerical Analysis}

Simulating the real health, performance and financial effects of WIH risk factors needs an appropriate estimation of WIH information. For this reason, we examine the developed optimization model's behavior according to WIH information, which were collected from manufacturing workers for the case of work-related low back pain and shoulder problems. These work-related MSD problems account for more than $30 \%$ of all compensated claims in Ontario, Canada. (e.g., WSIB 2010a). This approach supports our analysis to reproduce the work-related effects of WIH risk factors, which is taking place in a real working environment, for the sampled assembly system.

For numerical analysis, let us assume $c_{1 \tau}^{r}=\$ 20$ and $c_{1 \tau}^{o}=\$ 25$ per product. These costs are assumed to increase linearly by $\$ 5$ per product for each consecutive workstation. We also assume $s_{\tau}=\$ 165$ per outsourced product; $m e_{\tau}=\$ 74$ per day per ill worker; $i n w_{j}=\$ 40,000$ as the annual insurable wage per operator; and $w l_{j \tau}=\$ 20$ per hour. We initialize $d_{\tau}=2,600$ units (demand) for the first month, which is linearly increased by 50 units per month for the rest of the year. Furthermore, $b_{1 \tau}=\$ 5$ per stored products for the first buffer. This cost is also assumed to linearly increase by $\$ 2$ per product for each consecutive buffer. A healthy operator is assumed to work with $100 \%$ of his/her productivity level ( $z_{j \tau}^{h}=12$ parts per hour). However, when the operator is in the pain state, the productivity will be dropped to a lower level. To estimate an accurate result, the authors did extensive literature review to deterine the productivity loss due to MSD. The results show that the productivity loss due to workrelated health problems can vary from 3\% to 34\% (e.g., Allen et al. 2005; Meerding et al. 2005; Oxenburgh and Marlow 2005; Lerner et al. 2009; Martimo et al. 2009). For this study, it is cautiously estimated as $16.6 \%$, which is the average of productivity losses reported on previous HF studies.

\subsubsection{Case I: Low Back Pain}

Low Back Pain (LBP) is a common work-related health problem and a frequent cause of disability claims (WSIB 2010a). In this section, the developed model is tested by evaluating WIH risk factors for LBP, measured from an automotive company in Ontario, Canada Kerr 1997). Kerr (1997) studied the contribution of WIH risk factors to LBP by comparing subjects 
who reported LBP to nurse stations, "the cases", with workers who did not report but are otherwise similar, "the controls". Results show that WIH risk factor measures of "the cases" are higher than "the controls". In general, the most important WIH risk factors are identified taking into account their contributions in developing work-related health problems. In HF studies like Kerr (1997), these contributions are determined by comparing the health-related effects of the given risk factors between "the cases" and "the controls" groups. A higher contribution for a given risk factor shows the more importance of the the risk factor in developing LBP. For this analysis, the healthy state is denoted by 0 and the low back pain state with productivity loss is denoted by 1 .

A sub set of 7 physical and psychosocial WIH risk factors which were found to contribute to LBP including:

a) Peak hand force (loading) which is defined as the maximum average lifting pars weight over a designated interval of the time and usually is measured in Kilogram scale.

b) Peak shear force which is defined as the maximum average of shear forces acting on L4/L5 lumbar spine.

c) Cumulative compression loading which can be understood as the integral of instantaneous loads on L4/L5 spine over a shift. It is scaled according to Mega Newtons seconds per full-time shift.

d) Job control which is defined as the perception of employees regarding their job requirements, task repetitions involved in their jobs and ability to make decisions on the way they work.

e) Perceived physical exertion which measures the perception of employees regarding physical demands of their jobs.

f) Social environment which is defined as the operators' perception of the social atmosphere at the workplace.

g) Over-educated level which is about the perception of workers' education level compared to that of their co-workers performing similar jobs.

\section{Step 1}

From Equation (5.1), based on LBP work-related risk factors, parameters of the Logistic regression were determined. Table 5.1 presents their values.

Physical risk factors are measured according to on-site workplace observation or biome- 


\begin{tabular}{|c|c|c|c|c|}
\hline Risk factors & $\begin{array}{l}\text { Measures } \\
(s t d)\end{array}$ & $\begin{array}{c}\text { Odds Ratio(ODR) } \\
95 \% \text { CI }\end{array}$ & $\begin{array}{l}\text { Coefficient correlation } \\
\beta_{n j}=(\ln \text { ODR }) / a_{n j}\end{array}$ & $\begin{array}{c}\text { Constant coefficient } \\
\qquad \alpha_{0 j}\end{array}$ \\
\hline Peak hand load $(K g)$ & $14.3(12.4)$ & $1.8(1.15-2.87)$ & 0.035 & \multirow{7}{*}{-1.339} \\
\hline Peak shear $N$ & $354(159)$ & $1.9(1.16-3.17)$ & 0.003 & \\
\hline $\begin{array}{c}\text { Cumulative compression } \\
M N \text { /shift }\end{array}$ & $\begin{array}{c}12.2 \\
(0.665)\end{array}$ & $1.9(1.26-2.93)$ & $8.2 \mathrm{E}-08$ & \\
\hline Job control & $56.5(19.2)$ & $1.9(0.89-4.09)$ & -0.020 & \\
\hline Perceived exertion & $6(1.6)$ & $3.2(1.91-5.70)$ & 0.582 & \\
\hline Social environment & $12.4(1.7)$ & $2.8(1.42-5.84)$ & -0.343 & \\
\hline Over educated level & $($ yes $/$ no $)=1 / 0$ & $2.3(1.09-5.15)$ & 0.833 & \\
\hline
\end{tabular}

Table 5.1: Low back pain WIH risk factor measures and parameters used in the Logistic regression

chanical simulation softwares in order to analyze body posture of employees at work, using methods validated in the WIH research. For the defined psychosocial risk factors considered in this dissertation validated questionnaires have been designed by previous ergonomics studies to measure them with Likert-type scale (e.g., Perlin and Schooler 1978; Borg 1982; Karasek et al. 1998; Jencks et al. 1998; Vandergrift et al. 2012). For instance, perceived exertion can be measured by Borg scale. According to this measurement tool, the perceived physical demand of working tasks is scaled from 0 to 14 . While 0 is the lowest exposure level showing "very very light physical demand", 14 is the highest exposure level of the physical exertion risk factor showing that an operator perceives "very very high physical demand" to completing working tasks in the workplace. There are similar scaling approaches to measuring the other psychosocial risk factors. For another example, lets consider social environment, which was measured according to Job Content Questionnaire (JCQ), developed by Karasek et al. (1998). According to the JCQ, the perception of an operator from the social atmosphere of the workplace can be measured by employing 4 point likert-type scale questions. Each question has a set of ordinal response options, ranging as: "Strongly Agree: 1", "Agree: 2", "Disagree: 3", and Strongly Disagree: 4". The exposure level of the social environment risk factor is determined taking into account the summation of quantitative values, which are associated with the response options of each question and selected by the responders when answering all social environment questions. With regard to combining the social environment questionnaire items, the corresponding exposure level has a range from 4 to 16 in this thesis numerical analysis. While 4 shows the strong disagreement of the operator regarding the pleasant and socially attractive atmosphere of the workplace, 16 indicates the strong agreement of the operator. The examples of question 
items used to measure psychosocial risk factors are presented in the appendix.

$\beta_{n j}$ is the correlation coefficient of a given WIH risk factor, developing work-related low back pain. It is estimated with respect to odds ratio value, determined by Kerr (1997). An odds ratio shows a relative measure of a given WIH risk factor's health effect. Epidemiological studies such as Kerr et al. (2001) usually quantify the contribution of possible WIH risk factors with work-related health problems by comparing subjects who reported health problems, "the cases", with workers who did not report but are otherwise similar, "the controls". Hence, an odds ratio is interpreted as how much more likely it is that an employee exposed to a risk factor will report pain by comparing "the cases" with "the controls" results (Kerr 1997). Parameter $a_{n j}$ demonstrates the average difference of a given risk factor measured between "the cases" and "the controls" (Kleinbaum and Klein 2010).

Due to the non-randomized selection of "the cases", the constant coefficient, $\alpha$, of the Logistic regression formula must be adjusted, Equation (5.15), in order to calculate the transition probability of being in the pain state (King and Zeng 2002). The value of the initial constant variable of the Logistic regression, $\alpha^{\prime}$, is calibrated according to the combination of the population fraction of employees reporting low back pain, $\chi$, and the prevalence rate of employees reporting low back pain in the Kerr (1997) study, y'.

$$
\alpha_{0 j}=\alpha^{\prime}-\ln \left(\frac{1-\chi}{\chi} \times \frac{y^{\prime}}{1-y^{\prime}}\right)=-1.2-\ln (1.5 \times 0.766)=-1.339 .
$$

By extracting WIH information from the automotive study and compromising them with the defined health-states, the steady-state probabilities of the healthy state and the low back pain state with productivity loss are calculated for each operator according to Equations 5.2 . and $(5.3)$.

\section{Step 2}

In this step, the steady-state probabilities of being in the healthy and pain states are applied to the optimization model. The model is coded based on integer programming and solved by using IBM ILOG Cplex 12.1. Three different scenarios are developed to test the effects of low back pain WIH risk factors at the system level performance analysis. 


\section{Scenario 1}

This scenario aims to evaluate the impact of a WIH risk factor on the optimum total cost of the assembly system individually. For this reason, at each time, the risk factor is changed within a distance of \pm 3 standard deviation (std) from its mean value for all operators, while the other LBP risk factors are set to their mean levels, as measured by Kerr (1997) study. Consequently, the steady-state probability of each health-state is calculated for the operators at the time and the total cost model is minimized with the new probability inputs. This procedure is repeated for all WIH risk factors.

Figures (5.1) and (5.2) describe the behavior of the total cost versus the physical and psychosocial risk factor changes. They also compare the values of the total cost, minimized with and without including WIH effects. In the model without WIH effects, the operators produce products at a fixed rate. We assume that they are $100 \%$ productive. Furthermore, the objective function of the optimization model does not include indirect OHS, and wage loss costs, since the model without including WIH effects cannot track the effect of WIH risk factors.

In general, the negative effect of a given risk factor increases the chance of injury and entering to the pain state for the operators who are working in the assembly line. As a result of a lower productivity level in the pain state, the expected production quantity of the assembly line decreases and more products are supplied by subcontractors at a higher cost. Moreover, medical treatment expenses, insurance premium, and OHS costs increase with the higher chance of being in the pain state. Consequently, the total cost of the assembly line is minimized at a higher amount.

Figure (5.1 $)$ ) shows the behavior of the total cost by changing the peak load measure between 1.9 and $63.9 \mathrm{~kg}$. The results demonstrate that when the operators use a higher hand-force to lift, push or pull heavier objects during working shifts, there is a higher probability for them to be injured and thus being in the pain state with the lower productivity level. This probability varies from $21.2 \%$ to $58.8 \%$ by increasing the weight of the objects from 1.9 to $63.9 \mathrm{~kg}$ while the other WIH risk factors are set to their mean exposure levels, described by Table 5.1. Hence, the production rate of the line decreases and more products are outsourced to compensate the operators' health-related production losses. Furthermore, OHS costs increase due to a higher chance of being in the pain state and using medical treatments and compensation benefits by the operators. 


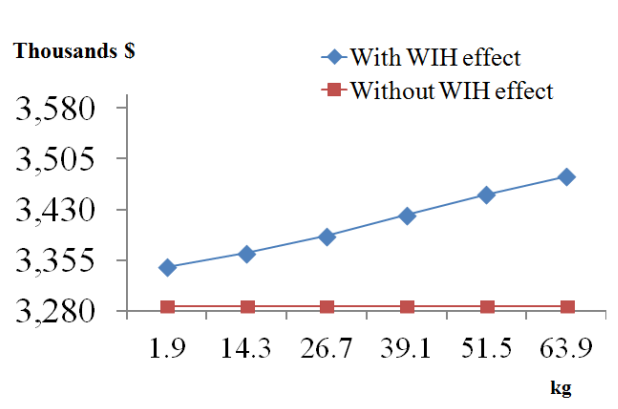

a) The total cost vs. Peak load changes

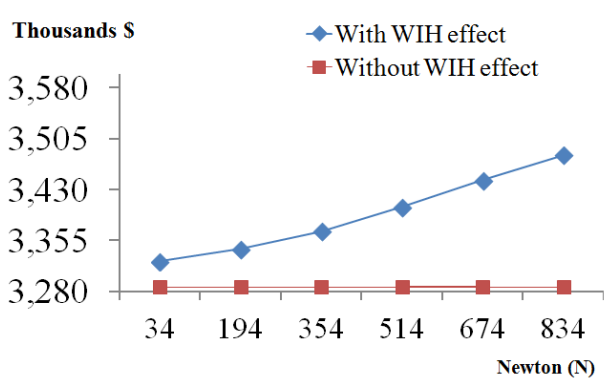

b) The total cost vs. Peak shear changes

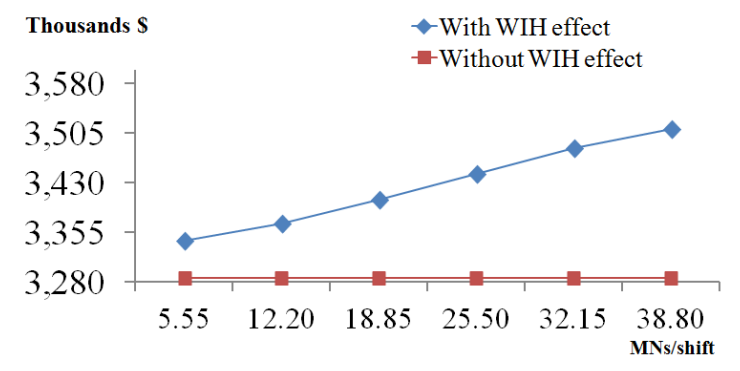

c) The total cost vs. Cumulative compression changes

Figure 5.1: The behavior of the minimum total cost versus LBP physical risk factor changes

When the operators lift objects with the average of $1.9 \mathrm{~kg}$, the major cost element arises from the expected production cost in regular time at the healthy state which is equal to $\$ 1,669,250$. Furthermore, 509 products are outsourced which incur a subcontracting cost of $\$ 83,985$. By increasing the peak load level, operators start to produce more products in the pain state while more products are also supplied by subcontractors. Consequently, at the maximum peak load level, the major cost element arises from the expected regular time production cost when the operators are in the pain state, which is determined to be $\$ 1,037,760$. The subcontracting cost also reaches its highest amount of $\$ 377,520$.

Since the model considers 4 operators, the insurance premium cost allocates the lowest portion of the total cost. It is equal to $\$ 3,717$ when the peak load is $1.9 \mathrm{~kg}$. However, the negative effect of peak load causes this cost element to increase. It becomes $\$ 4,008$ when the peak load level is set to $63.9 \mathrm{~kg}$. Consequently, the total cost of the assembly line increases from $\$ 3,347,014$ to $\$ 3,486,103$ by increasing the peak load level. There is a similar trend for the total cost, when the model examines the effect of other physical WIH risk factors, shown 
in Figures $(5.1 \mathrm{~b}$ and $\mathrm{c})$.

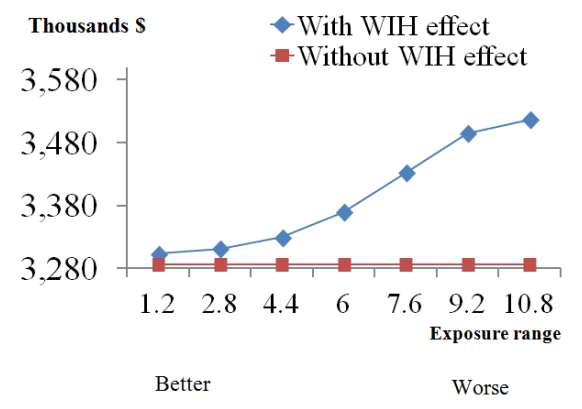

a) The total cost vs.

Perceived exertion changes

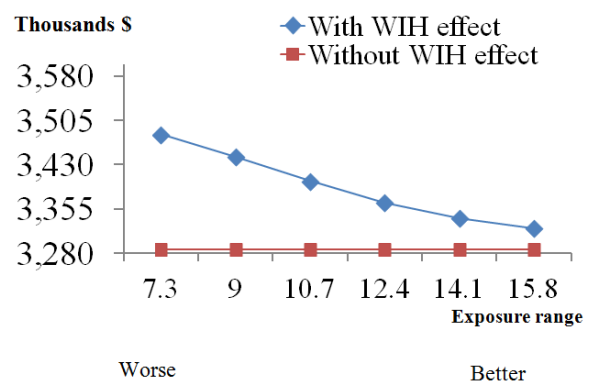

c) The total cost vs.

Social environment changes

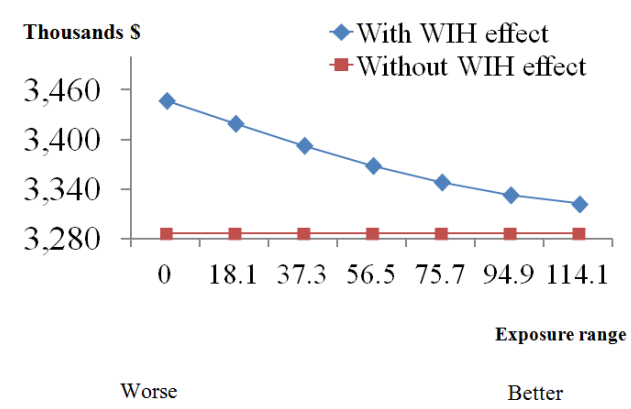

b) The total cost vs.

Job control changes

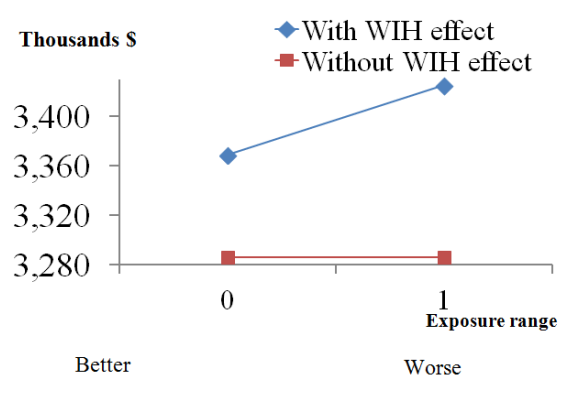

d) The total cost vs. Over-educated perception changes

Figure 5.2: The behavior of the minimum total cost versus LBP psychosocial risk factor changes

Figure (5.2 3 ) demonstrates the impact of the operators' perceived exertion on the total cost of the assembly line. The analysis of this psychosocial risk factor effect shows that the total cost increases from $\$ 3,303,340$ to $\$ 3,516,192$ when the perceived exertion level is varied from 1.2 to 10.8. At the lowest level, operators perceive minimum intensity level regarding the physical demand of their jobs accomplished during working shifts. Hence, there is the lowest probability for them to be in the pain state, $2.7 \%$, and work with a lower productivity level. However, by increasing the exposure level, the operators perceive a higher physical demand to accomplish their jobs with an associated increase in the chance of being in the pain state. At the highest exposure level, the probability of the pain state becomes $66 \%$. Consequently: a) the production rate of the line decreases; b) more products are ordered to subcontractors; and c) OHS costs increase. 
At the lowest physical exertion level, most of the demand is fulfilled by the assembly line and the operators produce most of the products in the healthy state. Hence, the expected production costs in the healthy state and pain state and subcontracting expenses are determined to be $\$ 3,199,060, \$ 75,696$ and $\$ 15,180$, respectively. When the operators perceive a higher physical demand to accomplish their jobs, the probability of being in the pain state increases and the production rate of the line decreases due to the health-related operators' productivity loss. Furthermore, the operators use medical treatments and compensation benefits more than before which results in a higher insurance cost. Therefore, at the highest exertion level, the expected production cost in the healthy state, pain state and subcontracting expenses become $\$ 1,159,924, \$ 1,871,908$, and $\$ 444,840$ respectively.

The behavior of the total cost versus psychosocial risk factor changes, shows a negative slope when the model evaluates the effect of job control and social environment. This negative slope is due to measurement tools and scaling of the given psychosocial risk factors. For example, job control is scaled from 0 to 114.1. At the zero level, the operators perceive minimum control over their jobs and, subsequently, they have the highest probability of being in the pain state. Therefore, the total cost of the assembly line is equal to $\$ 3,447,057$. By increasing the risk factor's exposure level, they perceive a higher level of control over their jobs, while they do various tasks with a lower repetition level to assemble products. Consequently, the probability of being in the pain state decreases. The total cost becomes $\$ 3,322,800$ when the exposure level of job control is 114.1 .

For more details, Table 5.2 shows the results of the developed model for the examples of the physical and psychosocial low back pain WIH risk factors. The results have been compared to the model without including WIH effects. The table includes the variations of steady-state probabilities of being in each health state, insurance premium, subcontracting expenses and the minimized total cost of the assembly line. The mechanism of perceived physical exertion, job control, social support and over-educated in developing MSD were investigated in previous studies (e.g., Carayon et al. 1999; Devereux et al. 1999; MacFarlane and Silman 2000). In general, psychological risk factors can increase muscle tensions which are intensifying physical strain over human body. This increased physical strain subsequently contributes to the appearance of MSD symptoms (e.g., EUOSHA 2008, 2011). 


\begin{tabular}{|c|c|c|c|c|c|c|}
\hline Risk factors & Exposure level & $\pi_{j \tau}^{p}$ & $\pi_{j \tau}^{h}$ & $\mathrm{IN}(\$)$ & Subcontracting $(\$)$ & Total cost $(\$)$ \\
\hline $\begin{array}{l}\text { Peak hand load } \\
(\mathrm{Kg})\end{array}$ & $\begin{array}{c}1.9 \\
14.3 \\
26.7 \\
39.1 \\
51.5 \\
63.9 \\
\end{array}$ & $\begin{array}{l}0.212 \\
0.283 \\
0.363 \\
0.445 \\
0.522 \\
0.588 \\
\end{array}$ & $\begin{array}{c}0.788 \\
0.717 \\
0.637 \\
0.555 \\
0.478 \\
0.478 \\
\end{array}$ & $\begin{array}{c}3,717.3 \\
3,773.57 \\
3,835.01 \\
3,900.8 \\
3,955.71 \\
4,007.81 \\
\end{array}$ & $\begin{array}{c}83,985 \\
124,410 \\
178,035 \\
247,005 \\
316,140 \\
377,520 \\
\end{array}$ & $\begin{array}{l}3,347,014 \\
3,368,767 \\
3,395,497 \\
3,427,638 \\
3,458,661 \\
3,486,103 \\
\end{array}$ \\
\hline $\begin{array}{l}\text { Peak Shear } \\
\text { (Newton) }\end{array}$ & $\begin{array}{r}34 \\
194 \\
354 \\
514 \\
674 \\
834 \\
\end{array}$ & $\begin{array}{l}0.127 \\
0.195 \\
0.283 \\
0.385 \\
0.486 \\
0.575 \\
\end{array}$ & $\begin{array}{l}0.873 \\
0.805 \\
0.717 \\
0.615 \\
0.514 \\
0.425 \\
\end{array}$ & $\begin{array}{c}3,652.35 \\
3,708.8 \\
3,773.57 \\
3,854.72 \\
3,930.18 \\
4,000.64 \\
\end{array}$ & $\begin{array}{c}45,375 \\
77,055 \\
124,410 \\
197,175 \\
282,480 \\
365,640 \\
\end{array}$ & $\begin{array}{c}3,324,307 \\
3,342,911 \\
3,368,767 \\
3404493 \\
3,443,760 \\
3,480,838 \\
\end{array}$ \\
\hline Perceived exertion & $\begin{array}{c}1.2 \\
2.8 \\
4.4 \\
6.0 \\
7.6 \\
9.2 \\
10.8 \\
\end{array}$ & $\begin{array}{c}0.027 \\
0.064 \\
0.144 \\
0.283 \\
0.459 \\
0.608 \\
0.66 \\
\end{array}$ & $\begin{array}{c}0.973 \\
0.936 \\
0.856 \\
0.717 \\
0.541 \\
0.392 \\
0.34 \\
\end{array}$ & $\begin{array}{c}3,575.55 \\
3,603.26 \\
3,664.7 \\
3,773.57 \\
3,906.62 \\
4,023.17 \\
4,060.23\end{array}$ & $\begin{array}{c}15,180 \\
24,420 \\
3,329,366 \\
124,410 \\
256,080 \\
397,320 \\
444,840 \\
\end{array}$ & $\begin{array}{c}3,303,340 \\
3,310,717 \\
3,327,750 \\
3369010 \\
3,432,043 \\
3,495,043 \\
3,516,192 \\
\end{array}$ \\
\hline Job control & $\begin{array}{c}0 \\
18.1 \\
37.3 \\
56.5 \\
75.7 \\
94.9 \\
114.1 \\
\end{array}$ & $\begin{array}{l}0.496 \\
0.429 \\
0.355 \\
0.283 \\
0.218 \\
0.163 \\
0.119 \\
\end{array}$ & $\begin{array}{l}0.504 \\
0.571 \\
0.645 \\
0.717 \\
0.782 \\
0.837 \\
0.881 \\
\end{array}$ & $\begin{array}{c}3,937.86 \\
3,883.58 \\
3,831.68 \\
3,773.57 \\
3,723.65 \\
3,681.41 \\
3,645.5 \\
\end{array}$ & $\begin{array}{c}292,380 \\
228,855 \\
173,580 \\
124,410 \\
87,285 \\
60,225 \\
42,900 \\
\end{array}$ & $\begin{array}{c}3,447,057 \\
3419719 \\
3,393,270 \\
3,368,767 \\
3,348,778 \\
3,333,361 \\
3,322,800 \\
\end{array}$ \\
\hline Model without WIH effects & $\mathrm{N} / \mathrm{A}$ & 0 & 1 & 3,600 & 9,075 & $3,286,300$ \\
\hline
\end{tabular}

Table 5.2: Examples of WIH risk factor effects

\section{Scenario 2}

In this case, we study the effect of simultaneous variation of 2 risk factors on the total cost. At each time, two risk factors are changed within a distance of \pm 3 standard deviation (std) from their mean values while the other LBP risk factors are set to their mean measures. Consequently, the steady-state probability of each health-state is calculated for all operators at the time and the total cost is minimized with the new probability inputs. Figure $(5.3)$ show the joint effect of the examples of physical and psychosocial LBP risk factor combinations on the total cost.

Figure (5.3 ) demonstrates the behavior of the total cost versus the joint effect of peak load and cumulative compression changes. The peak load is changed from 1.9 to $63.9 \mathrm{~kg}$. At each exposure value assigned to the peak load level, the cumulative compression is also varied within a distance of \pm 3 std from its mean measure, as observed by Kerr (1997). Compared with the result of Scenario 1, in stead of a graph showing the effect of peak load changes on the total 

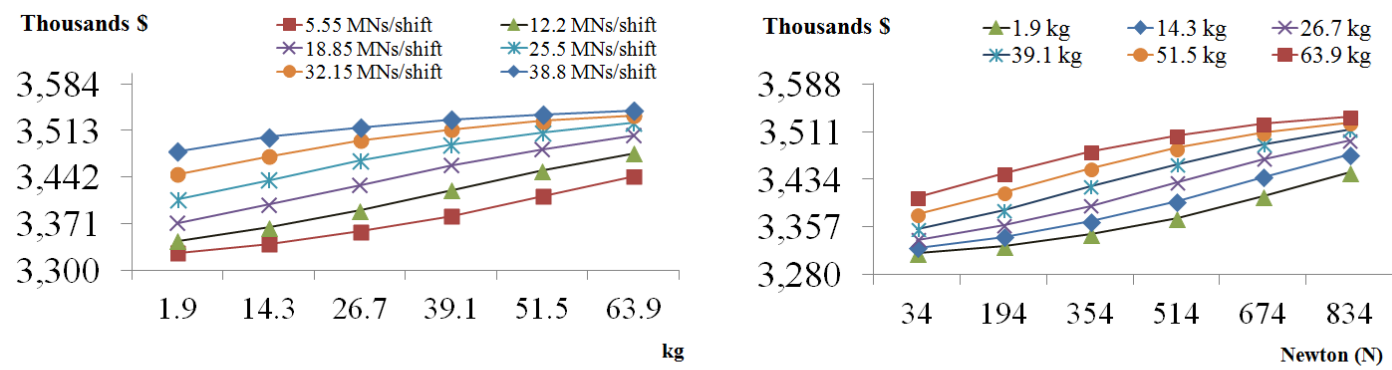

a)The total cost vs. Peak load changes with different cumulative compression levels

b)The total cost vs. Peak shear changes with different peak load levels

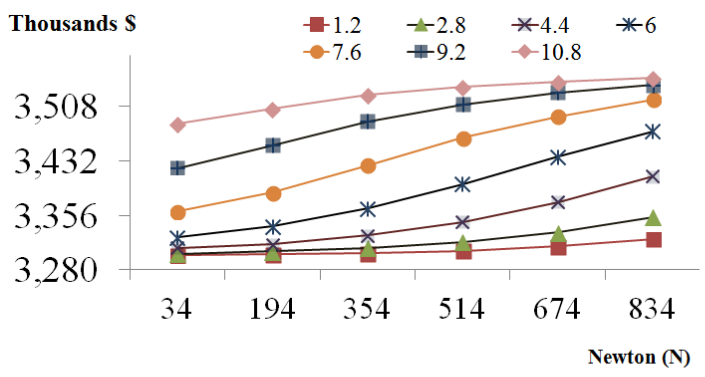

c) The total cost vs. Peak shear changes with different perceived exertion levels

Figure 5.3: The behavior of the minimum total cost versus LBP risk factor joint effects

cost, there are 6 graphs demonstrating the behavior of total cost versus the joint effect of peak load and cumulative compression changes. The lowest and highest amount of the total cost at each peak load level is determined by setting the cumulative compression to its lowest and highest exposure measures. For example, at $14.3 \mathrm{~kg}$ peak load level, the total cost changes from $\$ 3,341,931$ to $\$ 3,511,440$ when the cumulative risk factor is increased from 5.55 to 38.8 MNs/shift.

By setting peak load and cumulative compression to their highest exposure levels, $63.9 \mathrm{~kg}$ and $38.8 \mathrm{MNs} / \mathrm{shift}$, the operators will have the highest chance of being injured and enter to the pain state. With a $74.4 \%$ probability of being in the pain state, the production rate of the line decreases due to the lower productivity level of the operators in the pain state and subcontractors must provide 3,188 products to satisfy annual demand. Moreover, OHS costs reach its highest amount. Finally, the minimized total cost is equal to $\$ 3,551,070$. However, at the lowest peak load and cumulative compression exposure levels, $1.9 \mathrm{~kg}$ and $5.55 \mathrm{MNs} / \mathrm{shift}$, 
the total cost reduces to $\$ 3,327,080$.

The trend is similar for the other joint effects of the low back pain risk factors shown by Figures $(5.3 \mathrm{~b}$ and c). Compared with Scenario 1, the results of analysis in Scenario 2 also show the following relationship: $T C_{x_{1} x_{2}} \leq T C_{x_{1}}+T C_{x_{2}}$, where for any set of two low back pain risk factors, $\left(x_{1}, x_{2}\right), T C_{x_{1} x_{2}}$ is the total cost of the assembly line with their joint effect and $T C_{x_{1}}$ and $T C_{x_{2}}$, are the values of total cost calculated in Scenario 1 in order to evaluate $x_{1}, x_{2}$ effects individually.

\section{Scenario 3}

This scenario aims to investigate the effect of the WIH risk factor strengths in developing workrelated low back pain on the total cost of the system. For this reason, the value of the odds ratios shown by Table 5.1 is varied individually for all risk factors. An odds ratio indicates the strength of the association between a given risk factor exposure and work-related health problem. A higher odds ratio indicates a greater injury risk.

Similar to scenario 1, each risk factor exposure is changed within a distance of \pm 3 std from its mean measure at a time while the other LBP risk factors are set to their mean measures for all operators. Then, with the given odds ratio the risk factor, the steady-state probability of each health-state is calculated for all operators, and the total cost model is minimized with the new probability inputs. Then, the odds ratio varies around its value determined by Kerr (1997), shown by Table 5.1, and the procedure of changing the risk factor exposure level is repeated. Figures (5.4) and (5.5), show how the total cost behaves for different odds ratios.

Figure (5.4 a) shows the effect of variation in the odds ratio of the cumulative compression while the risk factor's level is changed from 5.55 to $38.8 \mathrm{MNs} / \mathrm{shift}$. Increasing the odds ratio raises the influence of the cumulative compression in developing low back pain. Hence, the probability of being in the pain state with productivity loss increases causing the total cost to be in a higher amount.

For instance, at $18.85 \mathrm{MNs} /$ shift exposure level, the total cost increases from $\$ 3,345,970$ to $\$ 3,479,840$ when the odds ratio is varied from 1.26 to 2.93 . With the value of 1.26 for the odds ratio, the chance of being in the pain state is minimum and the production is highest. Hence, only 497 products are supplied by subcontractors in a year. The company also spends the least amount of money for medical and compensation expenses and, subsequently, the cost 


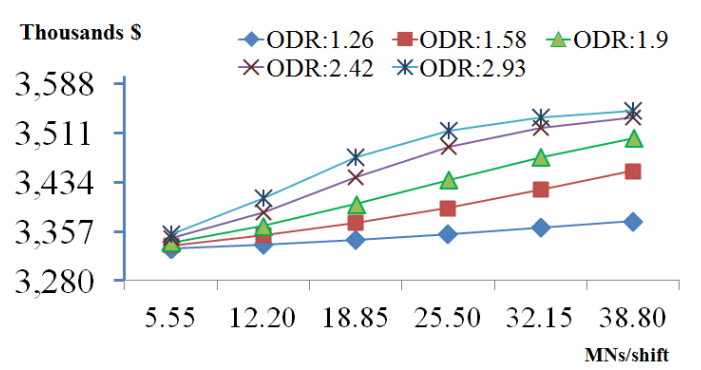

a) The total cost vs. Cumulative compression changes

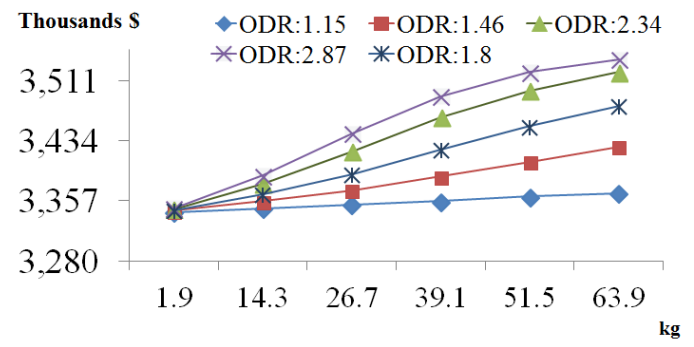

b) The total cost vs. Peak load changes

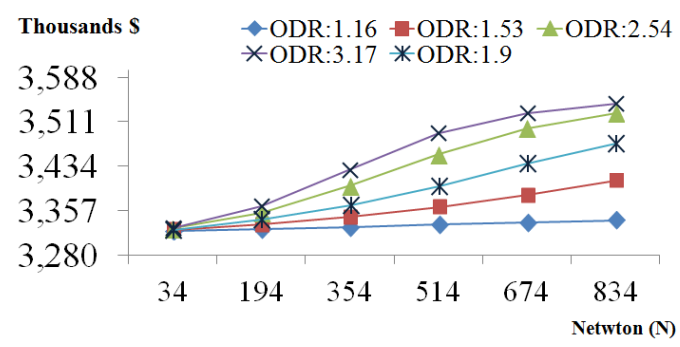

c) The total cost vs. Peak shear changes

Figure 5.4: The behavior of the minimum total cost versus changing the odds ratio (ODR) of physical risk factors

of insurance premium is estimated at $\$ 3,715$. However, by increasing the value of odds ratio to 2.93, the production rate of the line decreases due to the health-related productivity losses of the operators. Consequently, the number of products supplied by subcontractors have $343 \%$ increase and the insurance premium cost is estimated at $\$ 3,995$ due to the higher contribution of cumulative compression in developing low back pain. The same trend was found by varying the odds ratio of other LBP risk factors shown in Figures (5.4) and (5.5).

\subsubsection{Case II: Work-related Shoulder Disorders}

In this section, the effects of WIH aspects originated from work-related shoulder disorders in optimizing the performance of the serial assembly line are investigated. The primary WIH information is extracted from Sim et al. (2006). This study followed a cross-sectional methodology describing the relationship between work-related health problems such as shoulder pain with WIH risk factors. Sim et al. (2006) have investigated WIH risk factors via standard questionnaires while they have dichotomized their exposure levels with a binary measure: 1 


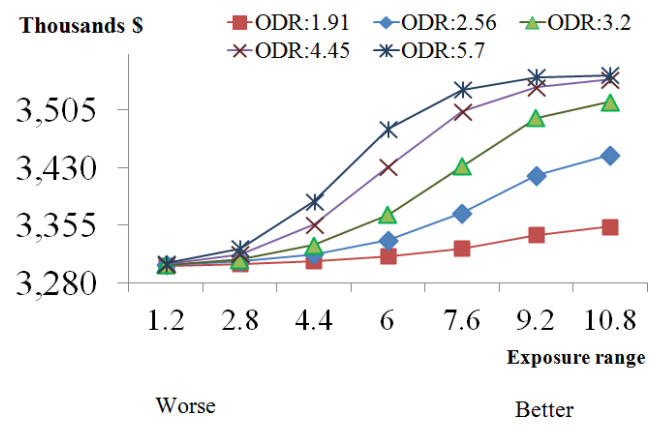

a) The total cost vs. Perceived exertion changes

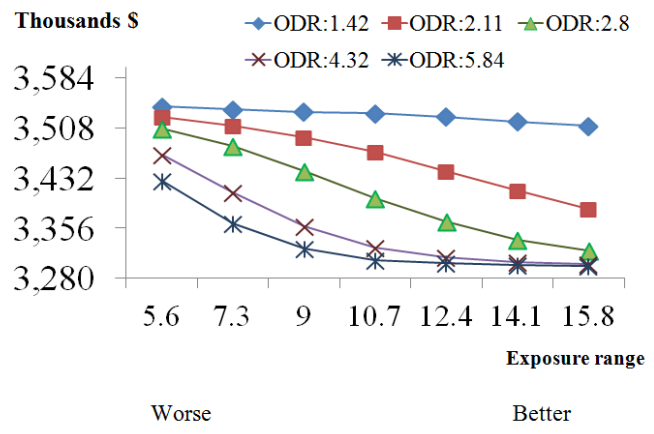

b)The total cost vs. Social environment changes

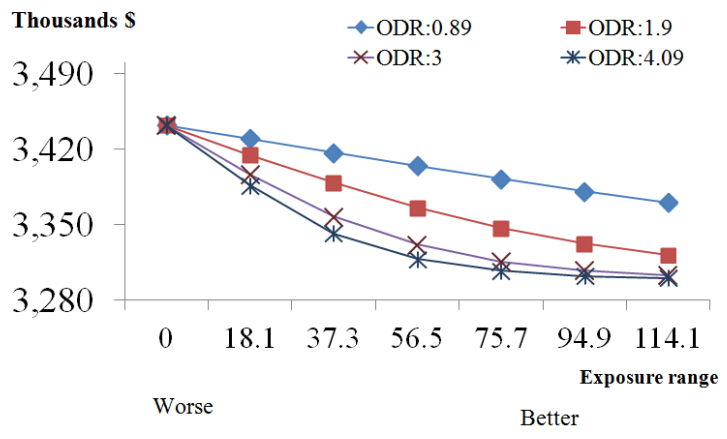

c) The total cost vs. Job control changes

Figure 5.5: The behavior of the minimum total cost versus changing the odds ratio (ODR) of psychosocial risk factors

(risk present) and 0 (risk absent), to calculate odds ratios. This is a common approach used in occupational epidemiology.

The features of the assembly line are the same as the previous scenarios. We define two work-related health-states, the healthy state and the shoulder pain with productivity loss state which are denoted by 0 and 1 , respectively. Three physical and two psychosocial WIH risk factors that have the main contributions to shoulder pain are used in the developed model. They include a) repeated lifting of heavy objects (RL); b) prolonged bending of neck (PB); c) working with one/both arms at shoulder height (AS) or above during the given working times; d) low job control (JC) as defined in LBP scenarios; and e) low supervisor support (SS), which is defined as the perception of operators regarding the received support and assistance from their supervisors and managers at work. 
Each operator of the sample assembly line has the same set of work-related shoulder pain risk factors with the dichotomous exposure value, 0 (not exposed) and 1 (exposed). In this analysis, different combinations of risk factors are investigated. That is, with a set of 5 binary physical and psychosocial risk factor measures (RL, PB, AS, JC, SS), there are $2^{5}$ combination starting from $(0,0,0,0,0)$ and ending by $(1,1,1,1,1)$. All combinations are considered in the two major steps of integrating WIH effects into the optimization model.

\section{Step 1}

In this step, our model determines the Logistic regression's output with respect to shoulder pain risk factor combinations. Then, the steady-state probabilities of being in the healthstates are calculated. Table 5.3 presents the parameters of the Logistic regression. $\beta_{n j}$ refers to

\begin{tabular}{|c|c|c|c|}
\hline Risk factors & Odds Ratio(ODR) & $\begin{array}{c}\text { Coefficient correlation } \\
\beta_{n j}=(\ln \text { ODR }) / a_{n j}\end{array}$ & $\begin{array}{c}\text { Constant coefficient } \\
\alpha_{0 j}\end{array}$ \\
\hline $\begin{array}{c}\text { Repeating/lifting heavy } \\
\text { object (RL) }\end{array}$ & 1.4 & 0.336 & \multirow{2}{*}{-1.191} \\
\hline $\begin{array}{c}\text { Prolonged bending } \\
\text { of neck (PB) }\end{array}$ & 2.1 & 0.742 & \\
\hline Arm at/above shoulder(AS) & 1.1 & 0.095 & \\
\hline Job control (JC) & 1.4 & 0.336 & \\
\hline Supervisor support (SS) & 1.2 & 0.182 & \\
\hline
\end{tabular}

Table 5.3: Shoulder WIH risk factor measures and parameters used in the Logistic regression

the correlation coefficient of a given WIH risk factor estimated with respect to the risk factor's odds ratio. With binary values for the WIH risk factors in cross-sectional studies like Sim et al. (2006), parameter $a_{n j}$ is equal to 1 (e.g., Hosmer and Lemeshow 2000). The $\alpha_{0 j}$ is a constant coefficient which is adjusted by Equation (5.16) to employ the Logistic regression output of cross-sectional studies as the transition probability. $\alpha^{\prime}$ is the initial constant coefficient resulting from the Logistic regression calculation. $\chi$ is the population fraction of workers reporting shoulder pain and $y^{\prime}$ is the rate of reporting shoulder problem in the Sim et al. (2006) study.

$$
\alpha_{0 j}=\alpha^{\prime}-\ln \left(\frac{1-\chi}{\chi} \times \frac{y^{\prime}}{1-y^{\prime}}\right)=-1.516-\ln (1.577 \times 0.464)=-1.191 .
$$

By adjusting the extracted HF information to the health-state definitions, the steady-state probabilities of the healthy and shoulder pain states are calculated for each operator by using 
Equations 5.2 and 5.3 .

\section{Step 2}

In this step, the steady-state probabilities of health-states are applied to the optimization model, and the total cost is minimized according to the WIH risk factor combinations. Finally, the behavior of the total cost resulting from the WIH risk factor effects is compared with the total cost generated without integrating WIH aspects, shown in Figure (5.6). Like the low back pain case study, in the model without WIH effects, the operators produce products at a fixed rate. We assume that they are $100 \%$ productive. Furthermore, the objective function of the optimization model does not include indirect OHS, and wage loss costs. Furthermore, we assume that all operators of the sample assembly line have the same risk factor levels to eliminate the effect of different risk factor levels of different workers in the sensitivity analysis and investigate risk factor effects at the system level optimization.

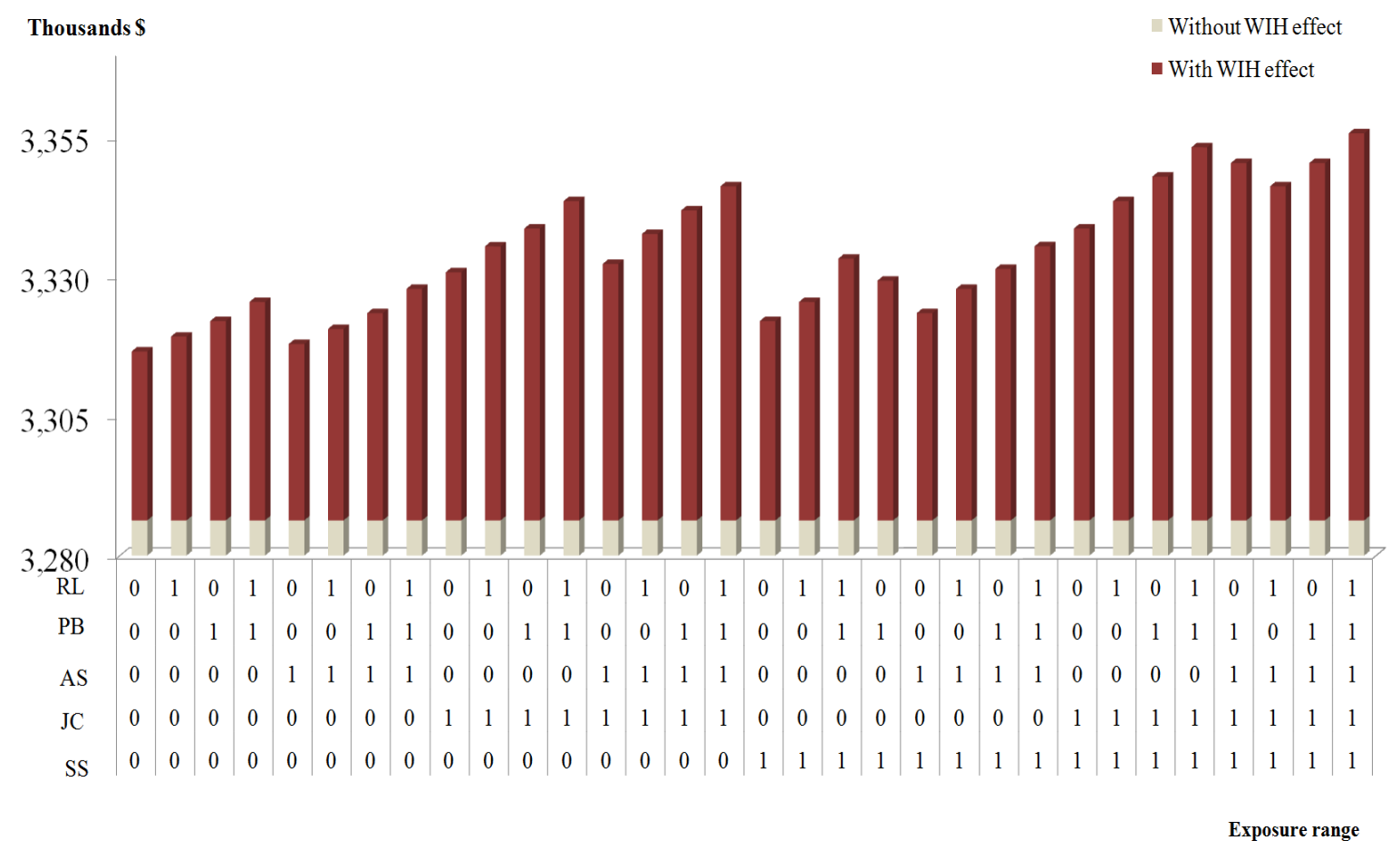

Figure 5.6: Minimum total cost vs. WIH shoulder pain risk factor combinations

The total cost changes from $\$ 3,317,184$ to the maximum $\$ 3,357,859$ according to different 
risk factor combinations. The results demonstrate that the maximum probability of being in the shoulder pain state is $24.9 \%$, when the operators of the assembly line receive little support from their supervisors, have a lower control over their job, work with arm above shoulder height, work with prolonged bending of their necks and lift heavy objects frequently during working shifts in a year. Consequently, the production rate of the line reduces due to health-related productivity loss of the operators and subcontractors supply 634 products within a year to meet the demand. Furthermore, the maximum insurance premium cost is equal to $\$ 3,745$ due to increased medical treatment and compensation expenses spent for operators because of work-related shoulder injury.

\subsection{Managerial Perspective}

This study follows a modeling approach to integrate work-related health hazards (WIH risk factors) and their health, performance and financial consequences in optimizing the performance of an assembly system. Compared with models without considering HF aspects, the results demonstrate several managerial advantages in terms of: reducing the underestimated value of the total cost of the system and examining the impact of the employees' performance variation due to work-related health problems on the optimized system performance and associated costs. Performance optimization models without HF considerations are unable to trace these issues.

Our findings show a considerable difference between the total cost values of the assembly line with and without integrating WIH risk factor effects. By incorporating WIH effects, the ergonomic design status of the workplace becomes an important feature of the operation system which leads to employee health variation. This work-related health change in the assembly system charges extra costs due to the lower performance of the employees. As shown by Table 5.2, these extra expenses make between $0.52 \%$ and $6.8 \%$ growth in the minimized total cost of the assembly line depending on the physical and psychosocial WIH risk factor levels of the work-related low back pain. The work-related cost growth becomes more significant in the presence of the joint effect of simultaneous WIH risk factor changes. For instance, shown by Figure (5.3 $)$, the total cost of the assembly line increases by $8 \%$ when the poor design of the workplace causes peak hand loading and cumulative compression to be at their highest exposure levels. In shoulder pain analysis, the minimized total cost of the assembly line increases from 
$0.9 \%$ to $2.2 \%$ when WIH risk factor combinations are changed from $(0,0,0,0,0)$ to $(1,1,1,1$, $1)$.

These total cost underestimations can significantly reduce the turn-over of the assembly system. For instance, if managers estimate $20 \%$ as the annual net profit of the assembly system, the negative WIH risk factor effects reduce this profit by between $2.5 \%$ and $34 \%$ when WIHrelated extra charges increase the total cost from $0.51 \%$ to $6.8 \%$ annually. These increased costs cannot be addressed by the performance models, which are ignoring WIH risk factor effects. Therefore, integrating WIH risk factor effects into system performance optimization will help managers better understand and manage economic consequences of WIH aspects in order to reduce the "hidden costs" of poor HF design in the workplace and replicate system behavior taking into account employee health-related performance variations.

Examining the behavior of OHS cost items, such as insurance premium, is another advantage of the developed optimization model. The results of this research show that there is a considerable relation between WIH risk factors and insurance premium cost. Due to the negative effect of WIH risk factors, the insurance premium cost paid by the company increases because of medical treatment and compensation benefit expenses of injured operators. As demonstrated by Table 5.2 , for instance, the ergonomic design of the workstations along with the physical demand of accomplishing assembly tasks, vary the chance of being injured from $21 \%$ to $59 \%$ with respect to peak load changes. Compared to the average annual insurance premium rate, $\$ 3,600$, paid for 4 operators by manufacturing companies in Ontario, Canada (WSIB 2010a), these work-related health variations increase the insurance premium between $1.44 \%$ and $11.13 \%$. Due to investigating only 4 operators for the assembly line in this study, these insurance cost growths have little contribution to the total cost of the system. However, They can make significant extra charges for medium and large manufacturing companies, which insure hundreds of employees in a year (e.g. WSIB 2010a).

Consequently, integrating WIH aspects into system performance optimization enables managers to: a) examine and predict WIH risk factor effects on insurance premium cost changes which are ignored in the optimization models without integrating WIH aspects; and b) deal with causes and consequences of insurance premium cost variations through evaluating the impact of WIH risk factors on employee capabilities, health conditions and OHS cost items such as medical and compensation expenses. 
Assisting managers to build a strategy for outsourcing production is another advantage of the developed model. In general, the results of this study show a remarkable difference in subcontracting cost values with and without integrating WIH risk factor effects. Without including WIH risk factor effects, only 55 units are outsourced annually. However, by integrating WIH risk factors and investigating the employees' productivity losses due to work-related health problems, outsourced production quantities increase dramatically. For instance, according to Scenario 1, the production variations of the system due to poor ergonomics design, increase the outsourced quantity between 275 and 2,216 units, when the peak shear exposure level is changed from $34 \mathrm{~N}$ to $834 \mathrm{~N}$. The negative effect of WIH risk factors also increases the dependency level of the company to subcontractors in order to fulfill customer demand. Subcontractors would affect the price and quality of production, resulting in reduction customer satisfaction and loosing market share. However, having a better ergonomic design in the workplace reduces the negative effect of WIH risk factors and decreases the dependency of the company on subcontractors. Consequently, the proposed model in this research assists managers to better understand their production situation due to HF effects. It also enables them to manage production variation and maintain a sustainable and healthy working environment.

Compared to the existing ergo-economic methods, the developed model in this study enables users to examine the contribution of WIH risk factors at the individual, workstation, and system levels. Most of the existing ergo-economics tools are developed based on workstation-level analysis, while they usually provide "snap-shot" approach in evaluating costs and benefits after and before an ergonomic intervention (e.g., Lahiri et al. 2005). These models ignore interactions between HF aspects (e.g. WIH risk factors) and other elements of the system (e.g. production capacity). Rose et al. (2013) discussed the shortcomings of the ergo-economics models in details. However, the proposed model combines HF aspects with other operational element of the system. It provides a better understanding for the financial consequences of HF aspects by integrating WIH risk factor effects in optimizing the performance of the system. It also replicates the behavior of the operation system with respect to the interactions among HF aspects and other elements of the system.

According to the purpose of this research, the developed model has been limited to include only work-related employee productivity loss. This assumption supported our analysis to comprehensively evaluate the effect of $\mathrm{HF}$ aspects at the system level performance optimization. 
But, the review of HF literature shows the negative effects of the poor ergonomic design of the system on employee error rate. Therefore, extending the optimization model by considering both work-related employee productivity loss and quality deficit is the primary goal of the author in the next research step. 


\section{Chapter 6}

\section{A modeling approach to integrating employee work-related productivity and quality variation into a serial system performance optimization models}

As discussed in previous chapters, Human Factors (HF) studies mostly focus on employee health and safety issues and are usually limited to performance measures at individual and workstation levels. On the other hand, Operations Management (OM) studies infrequently consider the impact of physical and psychosocial Work-related Ill Health (WIH) risk factors, which affect humans' health and their operational characteristics in terms of productivity loss and quality deficits. To span this research gap, this chapter presents a modeling approach, aiming to optimize an assembly line's total cost taking into account employee health-related productivity and quality variations. First, a three-health state Markov chain model is developed for each operator to determine steady-state probability of each defined health-state, which is varying based on physical and psychosocial WIH risk factor exposure levels. Then, the total cost model is minimized by incorporating the operators' health-related injury probabilities, operational and 
financial consequences (productivity loss and quality deficits) with other features of the system such as customer demand, inventory capacity and production costs. Compared to a model without HF effects, this study shows that the optimum total cost increases between $0.26 \%$ and $32 \%$ in the presence of different human factor aspects. Managers may take advantage of this research by having more opportunities to better deal with production variations due to employee $\mathrm{HF}$ (ergonomic)-related productivity and quality deficits, and develop a safe and efficient production plan via improving workplace ergonomic design.

The remainder of this chapter is organized as follows. Section 6.1 introduces the proposed model integrating WIH risk factor effects in the optimization of an assembly system's performance. Section 6.2 presents a modeling approach to consider human-related productivity loss and quality deficits. In Section 6.3, the proposed model is numerically tested with empirical HF data. In section 6.4, the benefits of investigating WIH effects are discussed from managerial perspective. Section 6.5 includes the limitation of the model and conclusion.

\subsection{Problem Description}

This section describes an operation system modeling approach, integrating WIH risk factors and their human-related operational and financial effects into a cost optimization model. Detailed numerical results will be provided in the numerical example. An assembly system with 4 workstations, 4 operators, and limited capacity buffers between the workstations is considered. This serial system is widely used in OM literature as well as manufacturing environment (e.g., Mendoza and Venturab, 2010; Shang, 2012). The assembly system operational details were described in previous chapters which are briefly reviewed in this section for the readers' convenience.

Product assembly starts from the first station. The operator brings product from the buffer of the previous station and carries out assembly tasks. Then, the incomplete product is sent to the next buffer. The final product is transferred from the fourth station into the inventory to satisfy demand. Due to work-related human errors, each operator may produce defective items which are categorized into rework and scrap products. The throughput of each station is inspected individually (e.g., Penn and Raviv 2006). Scrap products are rejected before being sent to the next station. Reworked products are returned to the same station during the next 
time period. Production is completed during regular and over-time hours. If the throughput of the assembly line cannot fully satisfy customer demand during the working shifts, subcontractors will fulfill the unmet demand. This study is focused on the operators' performance variations due to different WIH risk factor levels. It is assumed that machines used in the assembly line work perfectly and the production rate of workstations depends on the performance of the operators. Demand is known in each month, but it varies from one month to another.

Different tools are used by epidemiologists to evaluate the effects of WIH risk factors in developing Musculoskeletal Disorders (MSD) symptoms (e.g., Kerr et al. 2001; Ferguson et al. 2005; Eltayeb et al. 2007). They usually divide the study sample of a population into people with and without work-related pain (e.g., Dawson et al. 2009). The "in-pain" population includes individuals who become absent due to occupational health problems or stay at work while they are suffered from work-related pain effects (e.g., Kerr 1997). In this study, the operators' work-related health-states which are classified into 3 groups according to their health conditions include: healthy state when the operators do not have any MSD symptoms; work-related pain state for the operators staying at work and have MSD problem; absent from work state due to health problems. A new worker is replaced when an operator is in the absent state.

Based on the defined health-states, a three-state Markov chain is generated for each operator to calculate the steady-state probability of being in each health-state. The total cost model of the assembly line is then minimized over 12 consecutive months, by considering the operational effects of the operators' health-states.

The negative effects of work-related health problems on the performance of human beings are usually considered in terms of: producing defective items (quality deficit) and a lower production quantity level (e.g., Gonzalez et al. 2003; Yeow and Sen 2003; Meerding et al. 2005: Erdinc and Vayvay 2008; Erdinc and Yeow 2011). Being in each work-related health-state entails different health-related productivity levels and quality deficits.

In terms of the health-related productivity level, medicine and occupational health and safety studies have demonstrated on-job productivity loss for the "in-pain" operator who still stays at work (e.g., Schultz and Edington 2007; Schultz et al. 2009: Bergstrom et al. 2009: Johns 2010). This operational decline is referred as "presenteeism effect" (e.g., Meerding et al. 2005, Howard et al. 2009; Mannion et al. 2009). Furthermore, work-related MSD may also force the injured operator to temporarily leave the job and be replaced by a new worker. The new 
replacement workers usually have lower productivity than the healthy workers since they have a less competence and skills due to lack of working experience (e.g., Jaber and Khan 2010). For this study, it is assumed that an operator is $100 \%$ productive in the healthy state. However, the in-pain at work and replacement operators have the levels of productivity loss.

In terms of the quality of the production, in each health-state, the operator of the assembly system has a frequency of quality deficits due to human errors in operating production tasks. The source of the errors are categorized in two types for this research: A) factors related to systemic and common features of the production system, such as, improper use of a machine. These factors contribute to system-related inherent variability causing an unpreventable error rate, which is treated as a constant value in the model (e.g., Myszewski 2010); and B) factors related to the negative effects of working conditions (WIH risk factors) that contribute to excessive fatigue and subsequently musculoskeletal disorders (e.g., Yeow and Sen 2003, Kerst 2003, Fagarasanu and Kumar 2003; Feuerstein et al. 2004; Brown and Baker 2004, Prado-Lu

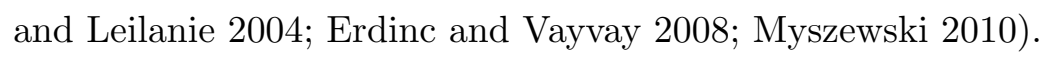

For this study, it is assumed that a healthy operator and a replacement worker have the same error rate which is mainly returned to the type "A" source of errors. However, those workers who have work-related MSD pain experience chronic (excessive) fatigue, generated mainly due to WIH risk factors (National Research Council|2001; Feuerstein et al.|2004). This excessive fatigue disrupts the performance of the operators by negatively affecting on their capabilities when they are completing assembly tasks during working shifts (e.g., Leung et al. 2006, El-Maraghy et al. 2008). This performance disruption has been shown in terms of increasing human errors (e.g., Giuntini 2000; Mallis et al. 2004; Chaffin et al. 2006; Ji et al. 2006; Myszewski 2010)). Hence, the operator in the pain-state has a higher rate of errors and quality problem than the healthy state. This error rate depends on the HF design of the workstation, resulting different WIH risk factors (working conditions) and fatigue levels.

\subsection{Major Steps in The Modeling Approach}

Our modeling approach in this research employs empirical based results of previous HF studies and common statistical methods used by epidemiology studies, in order to replicate the effect of WIH risk factors, occurring in a real working environment. It includes three major steps. 
In the first step, a connection is made between the exposure level of WIH risk factors and the 3-state Markov chain elements by using the Logistic regression technique. The results of this step enables us to determine the steady-state probability of being in each defined workrelated health-state. In the second step, the probability of producing defective products due to employee errors at each health-state is determined based on the concept of fatigue and quality deficits, taking from reliability theory. In the third step, the total cost model of the assembly line is optimized taking into account the results of previous steps.

\subsubsection{Step1: Health-State Markov Chain Calculation}

In this step, the continuous Markov chain concept is employed to approximate the steady-state probability of each health-state. First, a method is developed to estimate the rate of transferring from one health-state to another, in the presence of different WIH risk factor levels. Then, the generator matrix rate of the 3-health-state Markov chain, $\mathrm{Q}$, is created for each operator in order to determine the steady - state probabilities, shown by Equations (6.1) and (6.2).

$$
\begin{gathered}
Q=\left[\begin{array}{ccc}
-\left(\gamma_{j \tau}^{h w}+\gamma_{j \tau}^{h a}\right) & \gamma_{j \tau}^{h w} & \gamma_{\tau}^{h a} \\
\gamma_{j \tau}^{w h} & -\left(\gamma_{j \tau}^{w h}+\gamma_{j \tau}^{w a}\right) & \gamma_{j \tau}^{w a} \\
\gamma_{j \tau}^{a h} & \gamma_{j \tau}^{a w} & -\left(\gamma_{j \tau}^{a h}+\gamma_{j \tau}^{a w}\right)
\end{array}\right] \\
Q\left[\begin{array}{c}
\pi_{j \tau}^{h} \\
\pi_{j \tau}^{w} \\
\pi_{j \tau}^{a}
\end{array}\right]=\left[\begin{array}{l}
0 \\
0 \\
0
\end{array}\right] \quad \text { and, } \\
\end{gathered}
$$

Logistic regression is a statistical method in the epidemiology research field, which is applied to examine the influence of various known WIH factors on an operator's health outcomes. The output of the Logistic regression expresses the occurrence chance of being "in-pain"; or becoming absent due to injury, given the exposure level of WIH risk factors. In other words, with the specified risk factor levels, Logistic regression estimates the proportion of individuals having work-related health problems, from a population which is initially assumed to be healthy (e.g., Hosmer and Lemeshow 2000; Kleinbaum and Klein 2010). Equation (6.3) shows the Logistic function formula calculating the chance (proportion) of entering to the "pain" and "absent" states from the healthy state. 


$$
v_{j t}^{\bar{h}}=\frac{\exp \left[\sum_{y=1}^{n} \beta_{y j} X_{y j}+\alpha_{0 j}\right]}{1+\exp \left[\sum_{y=1}^{n} \beta_{y j} X_{y j}+\alpha_{0 j}\right]} \quad \text { where, } \quad \bar{h}=\{w, a\}
$$

For this study, the elements of the three-state Markov generator matrix rate are estimated according to the Logistic regression output.

First, Equation (6.4) is applied to convert the output of the Logistic regression (proportion value) to a cumulative rate. By this conversion, it is possible to estimate the rates of entering into the "in-pain" at work and the "absent" states from the "healthy" state, in the presence of different levels of WIH risk factors. Due to the annual analysis used in most epidemiology studies, $\lambda_{\bar{h} j t}$ is an annual rate, which is subject to change when the exposure levels of the investigated risk factors change.

$$
\lambda_{j t}^{\bar{h}}=\ln \left(\frac{1}{1-v_{j t}^{\bar{h}}}\right) \quad \text { where, } \quad \bar{h}=\{w, a\}
$$

Second, in order to determine the annual rate of entering to the "healthy" from the "in-pain" at work state, we equalize the healthy to the pain and the pain to the healthy transition probability formulas, which are developed according to the healthy-pain states continuous Markov chain approach and the healthy-pain states Logistic based Markov approach (Azzalini 1994), shown by Equations (6.5) and (6.6). This modeling method enables the author to generate the Markov chain transition rates from the Logistic regression output.

$$
\begin{gathered}
\frac{\lambda_{j t}^{\bar{h}}}{\lambda_{j t}^{\bar{h}}+\mu_{j t}^{\bar{h}}}-\frac{\lambda_{j t}^{\bar{h}}}{\lambda_{j t}^{\bar{h}}+\mu_{j t}^{\bar{h}}} \exp \left[-\left(\lambda_{j t}^{\bar{h}}+\mu_{j t}^{\bar{h}}\right) t\right]=v_{j t}^{\bar{h}}\left(1-u_{j t}^{\bar{h}}\right) \quad \text { where, } \quad \bar{h}=\{w, a\} . \\
\frac{\mu_{j t}^{\bar{h}}}{\lambda_{j t}^{\bar{h}}+\mu_{j t}^{\bar{h}}}-\frac{\mu_{j t}^{\bar{h}}}{\lambda_{j t}^{\bar{h}}+\mu_{j t}^{\bar{h}}} \exp \left[-\left(\lambda_{j t}^{\bar{h}}+\mu_{j t}^{\bar{h}}\right) t\right]=\left(1-v_{j t}^{\bar{h}}\right)\left(1-u_{j t}^{\bar{h}}\right) \quad \text { where, } \quad \bar{h}=\{w, a\} .
\end{gathered}
$$

By setting $t=1$, both continuous Markov chain and Logistic based Markov approaches should result in the same transition probabilities. Hence, assigning an appropriate autocorrection value will enable the author to determine $\mu_{j t}^{w}$. A similar procedure is followed to determine the annual rate of entering to the healthy state from the absent state, $\mu_{j t}^{a}$. 
Third, the values of $\lambda_{j t}^{w}, \lambda_{j t}^{a}, \mu_{j t}^{w}$, and $\mu_{j t}^{a}$ are multiplied by $1 / 12$ to determine the monthly transition rates. A literature review by the author shows the lack of enough evidence in HF studies regarding a comprehensive analysis in investigating the effect of both physical and psychosocial WIH risk factors on the health state change between "pain" and "absent" states. Therefore, to complete the matrix rate for each operator in this study, the monthly rate of entering to the "absent" from the "pain" state and the rate of transferring from the "absent" state to the "pain" state are estimated based on the average of the associated rates, resulting from reviewing previous medical and HF literature (e.g., MacKenzie et al. 1998; Lotters et al. 2005; Mannion et al. 2009). These rates are equal to 0.0317 and 0.0339 for the MSD healthstates, respectively.

Finally, the steady-state probabilities are calculated for each operator based on the given risk factor exposure levels. These probabilities can be changed by varying the matrix generator transition rates.

\subsubsection{Step 2: Calculating The Probability of Making Errors}

This step aims to determine the probability of human errors, generated according to the defined work-related health-states. This probability allows the estimation of the deficient items produced by the operators during the given time periods. In the presence of different numerical methods attempting to estimate human error occurrence, this study follows the concept of the failure function, the Weibull distribution, taken from reliability theory (e.g., Xie and Lai 1996 . Wang et al. 2002). The applicability of this function in addressing human errors has been discussed in previous literature (e.g., Giuntini 2000; Myszewski 2010).

The Weibull distribution enables the model to determine the constant error rate of the healthy and the new replacement operators, as well as the increasing error rate of the "inpain" at work operators. Equation (6.7) shows the Weibull probability density formula that determines the probability of making errors, $p_{j t}^{\bar{h}}$ by an operator in each health-state. 


$$
\begin{gathered}
f(t)=\frac{\zeta_{j}^{\bar{h}}}{\rho_{j}^{\bar{h}}}\left(\frac{t-\kappa}{\rho_{j}^{\bar{h}}}\right)^{\zeta_{j}^{\bar{h}}-1} \exp \left[-\left(\frac{t-\kappa}{\rho_{j}^{\bar{h}}}\right)^{\zeta_{j}^{\bar{h}}}\right] \quad \text { and, } p_{\bar{h} j t}^{\bar{h}}=\int_{0}^{t} f(t) d t=1-\exp \left[-\left(\frac{t-\kappa}{\rho_{j}^{\bar{h}}}\right)^{\zeta_{j}^{\bar{h}}}\right], \\
\bar{h}=\{h, w, a\} .
\end{gathered}
$$

To determine the constant error rate of the healthy and replacement operators, the shape parameter of Weibull distribution must be set to 1 (Myszewski 2010). Equation (6.8) demonstrates the corresponding probabilities.

$$
p_{j t}^{\bar{h}}=1-\exp \left[-\left(\frac{t-\kappa}{\rho_{j}^{\bar{h}}}\right)\right] \quad \text { where, } \quad \bar{h}=\{h, a\}
$$

However, being "in-pain" at work increases the slope of the Weibull distribution. The slope changes is impacted by physical and psychosocial WIH risk factors that result in the development of work-related MSD. This occupational health problem is mainly due to the development of muscle mechanical strain and chronic (excessive) fatigue (Feuerstein et al. 2004). Previous studies have demonstrated an increasing human error rate due to the work-related fatigue (e.g., Kopardekar and Mital 1994; Giuntini 2000; Ji et al. 2006; Michalos et al. 2013). In general, it is reasoned that an operator who has pain and continues his/her work has MSD-related excessive fatigue, reducing his/her muscle force capacity. This reduced force capacity causes physical exertion/physical discomfort which is limiting the operator's capability to perform his/her tasks at work correctly (e.g., Myszewski 2010; Barker and Nussbaum 2011; Dode 2011; Ham et al. 2012). Hence, entering to the muscle fatigue phase for the in pain operator causes a higher rate of errors than for a healthy worker. This health-related performance effect can increase the slope of the weibull function for the operator in the pain-state. Equation $(6.9)$ shows the "in-pain" probability of producing defects. The value of the $\zeta_{j}^{\bar{h}}$ can be substituted by any values bigger than 1, which is related to the accumulated fatigue level. In this study, the increased error rate of the in-pain operator is modeled as a function of the level of accumulated excessive fatigue, resulted based on the given workload level.

$$
p_{j t}^{w}=1-1-\exp \left[-\left(\frac{t-\kappa}{\rho_{j}^{\bar{h}}}\right)^{\zeta_{j}^{\bar{h}}}\right] .
$$


For this study, Equation $(6.10)$ determines the average excessive fatigue level, $F_{j t}^{w}$, of the "in-pain" operator. In the absence of experimental research, there are several studies which are suggesting exponential function for the fatigue behavior (e.g., Konz 1998; Dode 2011; Jaber et al. 2013). This formula measures human's cumulative (excessive) fatigue based on the percentage of his/her maximum fatigue level. Theoretically, an employee who reaches the maximum of his/her fatigue level is unable to continue working. The exponential function makes a connection between the average fatigue level of the "in-pain" operator, and the corresponding working condition (workload) at the workplace, $\Theta_{j}^{w}$, which is proportional to WIH risk factor levels. Changing WIH risk factor levels will vary the workload for the operator, which is modifying the excessive fatigue level of the "in-pain" operator at work. Subsequently, the operator's rate of errors changes.

$$
F_{j t}^{w}=1-\exp \left[-\Theta_{j}^{w} t\right]
$$

To achieve feasible results from the outputs of Weibull and fatigue formulas, their parameters are set within acceptable ranges, determined based on existing studies (e.g., Bubb 2005). Due to work-related errors, the defective products include rework and scrap items. For this research, it is also assumed that the rate of producing scrap products is the same as rework products for each operator, as shown by Equation (6.11). The numerical details of the parameters are given in the numerical analysis section.

$$
p_{j t}^{\bar{h}}=2 p_{j t}^{\bar{h} r w}=2 p_{j t}^{\bar{h} s}, \quad \text { where }, \quad \bar{h}=\{h, w, a\}
$$

\subsubsection{Step 3: System Performance Optimization Model}

In this step, the total cost function of the assembly line is minimized with respect to the operators' health-related operational performance and subsequent financial effects. The developed optimization model is described as the following: 


$$
\begin{aligned}
\text { Min } & \sum_{j=1}^{4} \sum_{\tau=1}^{12} \sum_{\bar{h}} c_{j \tau}^{r} \pi_{j \tau}^{\bar{h}} P_{j \tau}^{\bar{h} r}+\sum_{j=1}^{4} \sum_{\tau=1}^{12} \sum_{\bar{h}} c_{j \tau}^{o} \pi_{j \tau}^{\bar{h}} P_{j \tau}^{\bar{h} o}+\sum_{j=1}^{4} \sum_{\tau=1}^{12} b_{j \tau} B_{j \tau}+\sum_{\tau=1}^{12} s_{\tau} S_{\tau} \\
& +\sum_{j=1}^{4} \sum_{\tau=1}^{12} \sum_{\bar{h}} c s_{j \tau} \pi_{j \tau}^{\bar{h}} p_{j \tau}^{\bar{h} s} P_{j \tau}^{\bar{h} r}+\sum_{j=1}^{4} \sum_{\tau=1}^{12} \sum_{\bar{h}} c s_{j \tau} \pi_{j \tau}^{\bar{h}} p_{j \tau}^{\bar{h} s} P_{j \tau}^{\bar{h} o}+I N+I C \\
& +\sum_{j=1}^{4} \sum_{\tau=1}^{12} \sum_{\bar{h}} c r_{j \tau} \pi_{j \tau}^{\bar{h}} p_{j \tau}^{\bar{h} r w} P_{j \tau}^{\bar{h} r}+\sum_{j=1}^{4} \sum_{\tau=1}^{12} \sum_{\bar{h}} c r_{j \tau} \pi_{j \tau}^{\bar{h}} p_{j \tau}^{\bar{h} r w} P_{j \tau}^{\bar{h} o} \\
& +\sum_{j=1}^{4} \sum_{\tau=1}^{12} \sum_{\bar{h}} w l_{j \tau}\left(\frac{\pi_{j \tau}^{\bar{h}} P_{j \tau}^{\bar{h} r}+\pi_{j \tau}^{\bar{h}} P_{j \tau}^{\bar{h} o}}{z_{j \tau}^{\bar{h}}}-\frac{\pi_{j \tau}^{\bar{h}} P_{j \tau}^{\bar{h} r}+\pi_{j \tau}^{\bar{h}} P_{j \tau}^{\bar{h} o}}{z_{j \tau}^{h}}\right)
\end{aligned}
$$

subject to:

$$
\begin{gathered}
\sum_{\bar{h}} \pi_{4 \tau}^{\bar{h}} P_{4 \tau}^{\bar{h} r}+\sum_{\bar{h}} \pi_{4 \tau}^{\bar{h}} P_{\bar{h} 4 \tau}^{\bar{h} o}+B_{4 \tau}+S_{\tau}-\sum_{\bar{h}} \pi_{4 \tau}^{\bar{h}} p_{4 \tau}^{\bar{h} r w} P_{4 \tau}^{\bar{h} r}-\sum_{\bar{h}} \pi_{4 \tau}^{\bar{h}} p_{4 \tau}^{\bar{h} r w} P_{4 \tau}^{\bar{h} o} \\
\quad-\sum_{\bar{h}} \pi_{4 \tau}^{\bar{h}} p_{4 \tau}^{\bar{h} s} P_{4 \tau}^{\bar{h} r}-\sum_{\bar{h}} \pi_{4 \tau}^{\bar{h}} p_{4 \tau}^{\bar{h} s} P_{4 \tau}^{\bar{h} o}-B_{4 \tau+1} \geq d_{\tau} . \\
+\sum_{\bar{h}} \pi_{j \tau}^{\bar{h}} P_{j \tau}^{\bar{h} r}+\sum_{\bar{h}} \pi_{j \tau}^{\bar{h}} p_{j \tau}^{\bar{h} o}-\sum_{\bar{h}} \pi_{j \tau}^{\bar{h}} p_{j \tau}^{\bar{h} r w} P_{j \tau}^{\bar{h} r}-\sum_{\bar{h}} \pi_{j \tau}^{\bar{h}} p_{j \tau}^{\bar{h} r w} P_{j \tau}^{\bar{h} o}+B_{j \tau}-B_{j \tau+1} \\
-\sum_{\bar{h}} \pi_{j \tau}^{\bar{h}} p_{j \tau}^{\bar{h} s} P_{j \tau}^{\bar{h} r}-\sum_{\bar{h}} \pi_{j \tau}^{\bar{h}} p_{j \tau}^{\bar{h} s} P_{j \tau}^{\bar{h} o}+\sum_{\bar{h}} \pi_{j+1 \tau-1}^{\bar{h}} p_{j+1 \tau-1}^{\bar{h} r w} P_{\bar{h} j+1 \tau-1}^{\bar{h} r} \\
+\sum_{\bar{h}} \pi_{j+1 \tau-1}^{\bar{h}} p_{j+1 \tau-1}^{\bar{h} r w} P_{j+1 \tau-1}^{\bar{h} o}-\sum_{\bar{h}} \pi_{j+1 \tau}^{\bar{h}} P_{j+1 \tau}^{\bar{h} r}-\sum_{\bar{h}} \pi_{j+1 \tau}^{\bar{h}} P_{j+1 \tau}^{\bar{h} o} \geq 0 .
\end{gathered}
$$

where, $\quad j=\{1,2,3\}$

$$
\begin{aligned}
& P_{j \tau}^{\bar{h} r} \leq s h_{j \tau}^{r} m_{j \tau} z_{j \tau}^{\bar{h}} . \\
& P_{j \tau}^{\bar{h} o} \leq s h_{j \tau}^{o} m_{j \tau} z_{j \tau}^{\bar{h}} .
\end{aligned}
$$




$$
\begin{gathered}
S_{\tau} \leq p c t_{\tau} d_{\tau} \quad \text { and } \quad 0 \leq B_{j \tau} \leq b m_{j \tau} . \\
P_{j \tau}^{\bar{h} r}, P_{j \tau o}^{\bar{h} o}, S_{\tau} \text { and } B_{j \tau} \geq 0 . \quad \text { and } \quad \text { are integers }
\end{gathered}
$$

The objective function, Equation (6.12), minimizes the total production, inventory, subcontracting, scraping, reworking and OHS costs. The production cost includes the expected production cost of all workstations, accumulated over 12 months. For each workstation, the expected production cost during period $\tau$ is determined by multiplying the unit production cost with the expected production quantity produced by the corresponding operator in regular and over times, according to his/her health-state performances. The inventory cost is determined according to the buffer levels multiplied by the unit inventory cost of the product, accumulated over 12 months. The subcontracting cost is incurred whenever demand is not met by production during the given time periods. OHS costs are considered in terms of insurance premium cost, indirect costs and wage loss cost. Since scrapped products are permanently rejected from the production chain, the per unit scrapping cost is assumed to be 2 times the per unit reworking cost.

Constraints 6.13 and 6.14 guarantee the flow of products through the serial assembly line. Demand is deterministic in each time period $\tau$. Constraints (6.15) and (6.16) are subject to the available time duration during each month. It is assumed that there are 22 working days in a month. Constraint (6.17) limits the subcontracting and buffer capacity levels. The model allows the company to outsource production up to 40 percent of demand in each time period. The maximum buffer capacity is equal to 10 products per buffer.

At the firm level, insurance premium cost includes medical and compensation expenses due to work-related pain and injuries. According to the OHS performance-sensitive approach, insurance premium rate is estimated with respect to medical treatments and compensation expenses (e.g., Riel and Imbeau 1998). This rate is multiplied by the number of the employees to calculate the total insurance premium of the assembly system. Equation (6.18) shows the insurance premium cost, IN, adjusted in a way to consider WIH effects.

$$
I N=4 A I N \sum_{j=1}^{4} \operatorname{inw}_{j}\left(\sum_{j=1}^{4} \sum_{\tau=1}^{12} \sum_{h=\bar{w}, a} m e_{\tau} \pi_{j \tau}^{\bar{h}} m_{j \tau}\right)
$$


The unit cost of the insurance premium, AIN, is defined as per dollar of medical treatment and compensation expenses credited per dollar of insured salaries. For this study, AIN and the average of the annual insurable salary per worker, $i n w_{j}$, are estimated and adjusted according to statistics data, collected from the WSIB and the Ontario Ministry of Labor reports (e.g., HRSDC 2010; WSIB 2010b).

Indirect OHS costs include all overhead expenses that the company spent due to workrelated health problems. These costs usually include personnel acquisition costs and training expenses. These costs are not usually specified as a separate financial item in a company's financial reporting. However, several studies have estimated that these expenses in comparison with medical care and benefit costs (e.g., Leigh 2011). The results show that these indirect costs vary between 1 and 10 times of the direct medical and benefit costs. The developed optimization model in this study uses the WSIB's more conservative estimation, 3 times, in Equation 6.19 to consider OHS indirect cost effects (WSIB 2010a).

$$
I C=3 \sum_{j=1}^{4} \sum_{\tau=1}^{12} \sum_{\bar{h}=w, a} m e_{\tau} \pi_{j \tau}^{\bar{h}} m_{j \tau} .
$$

"Wage loss" is another important OHS cost which is considered in the developed model. This cost represents the amount of payments for working hours which are lost due to the operators' health-related productivity loss during the given time periods.

\subsection{Numerical Example}

In this section, the developed model is numerically tested to illustrate the impact of WIH risk factors on the optimum total cost of the assembly system. The low back pain HF information, measured from an automotive company in Ontario, Canada (Kerr 1997) have been used for numerical analysis. It is assumed that all 4 operators have the same WIH low back pain risk factors measuring in the same way as described in the Kerr (1997) study. Table 6.1 shows the value of the optimization model's parameters and the corresponding assumptions, which are set in a way that the developed model becomes feasible.

For the numerical analysis, the healthy operator is assumed to be $100 \%$ productive. However, the in-pain and replacement operators have the levels of productivity loss. From HF and 
medicine literature, the health-related productivity loss can vary from $3 \%$ to $34 \%$ (e.g., Allen et al. 2005; Meerding et al. 2005, Oxenburgh and Marlow 2005; Lerner et al. 2009; Martimo et al. 2009; Jaber and Khan 2010). For this paper, the productivity loss for the "in-pain" and replacement operators are cautiously estimated as $16.6 \%$ and $10 \%$, respectively, determined based on the average productivity losses reported by previous studies. For the rest of this section, first, the Logistic regression, Markov chain, Weibull function and fatigue parameters are estimated. Then, different scenarios are investigated to evaluate the operational and financial effect of the WIH risk factors.

\begin{tabular}{|c|c|}
\hline Parameters & Description \\
\hline$c_{1 \tau}^{r}=\$ 20$ & It is linearly increased by $\$ 5$ per product for each consecutive workstation \\
$c_{1 \tau}^{o}=\$ 25$ & It is linearly increased by $\$ 5$ per product for each consecutive workstation \\
$c s_{1 \tau}=\$ 100$ & It is linearly increased by $\$ 5$ per product for each consecutive workstation \\
$c r_{1 \tau}=\$ 50$ & It is linearly increased by $\$ 5$ per product for each consecutive workstation \\
$m e_{\tau}=\$ 74$ & Determined according to the Ontario's ministry of labor report $($ HRSDC $\mid 2010)$ \\
$d_{1}=2,600$ units & It is linearly increasing by 50 units per month for the rest of the year \\
$b_{1 \tau}=\$ 5$ & It is linearly increased by $\$ 2$ per product for each consecutive buffer \\
$\rho_{j}^{w}=185$ & when all WIH risk factor are set to their mean values \\
$\zeta_{j}^{w}=1.5$ & when all WIH risk factor are set to their mean values \\
$F_{j t}^{w}=25 \%$ & when all WIH risk factor are set to their mean values \\
$\kappa=0$ & for all health-states \\
$\rho_{j}^{\bar{h}}=16,000$ & $\bar{h}=h, a$ \\
$\zeta_{j}^{\bar{h}}=1$ & $\bar{h}=h, a$ \\
$b m_{j \tau}=10$ units & for all workstations \\
$i n w_{j}=\$ 40,000$ & for each operator \\
$w_{j \tau}=\$ 20$ & for all health-states \\
$s_{\tau}=\$ 165$ & for all heath-states \\
$s h_{j \tau}^{r}=8$ hours & for all health-states \\
$s h_{j \tau}^{o}=4$ hours & for all health-states \\
$m_{j \tau}=22$ days &
\end{tabular}

Table 6.1: The value of the system parameters and the corresponding assumptions

\subsubsection{Major Steps in Evaluating Low Back Pain Risk Factor Effects}

The numerical experiment follows the 3 steps of the developed modeling approach. For this study, the WIH risk factors include: a) cumulative spinal compression loading. This physical risk factor is determined based on the integrated instantaneous loads on L4/L5 spine over a shift. It is usually measured in Mega Newton seconds per full-time shift (MNs/shift); b) peak hand loading, which is scaled according to the maximum force exerted in the shift. It is usually 
measured in Kilograms (kg); c) peak shear force is the maximum average of shear forces acting on L4/L5 lumbar spine, measured in newtons $(\mathrm{N})$; d) job control is a psychosocial risk factor measuring the perception of an operator regarding his/her job requirements, task repetitions and his/her role in making decisions; e) perceived physical exertion measures the perception of operators regarding physical demands of their jobs; f) social environment is considered as an operator's perception of the social atmosphere of the workplace; and g) over-educated level is about the perception of operators education level compared to that of their co-workers performing similar jobs. Physical risk factors are usually measured using field observation, force gauges and biomechanical modeling software (e.g., Watbak, University of Waterloo). Psychosocial risk factors are usually measured with standard psychosocial tool measurement questionnaires designed using a Likert-type scale (e.g., Karasek et al. 1998). More details were described in chapter 5 (page 50) with regard to scaling psychosocial risk factor exposure levels. The examples of questions are also presented in the appendix. The mean exposure levels of the given low back pain risk factors are equaled as the average levels observed by Kerr (1997) and Kerr et al. (2001).

\section{Step 1: Low Back Pain-related Markov Chain Calculation}

According to the available epidemiological data in the Kerr (1997) study, the Logistic regression, $\Lambda_{j t}$, determines the proportion of workers who reported low back pain including those either "in-pain" at work or those who become "absent" and replaced by new operators. Table 6.2 represents the parameters of the Kerr's Logistic regression.

The correlation coefficient, $\beta_{n j}$ shows the contribution of a given risk factor in developing work-related low back pain. It is estimated based on the relative measure, odds ratio, of the associated WIH risk factor. In case-control epidemiological studies such as Kerr et al., (2001), odds ratios demonstrate the relative contributions of possible WIH risk factors with work-related health problems by comparing subjects who have low back pain, "the cases", with workers who did not report the health issue but are otherwise similar, "the controls". These statistical indicators help users to understand how much more likely it is that an employee, who is exposed to the given risk factor levels will report pain via comparing "the cases" with "the

controls" results (e.g., Kerr et al. 2001). Parameter $a_{n j}$ shows the average difference of a given risk factor measured between "the cases" group and "the controls". 


\begin{tabular}{|c|c|c|c|c|}
\hline Risk factors & $\begin{array}{l}\text { Measures } \\
\text { std }\end{array}$ & $\begin{array}{c}\text { Odds Ratio(ODR) } \\
95 \% C I\end{array}$ & $\begin{array}{c}\text { Coefficient correlation } \\
\beta_{n j}=(\ln \mathrm{ODR}) / a_{n j}\end{array}$ & $\begin{array}{c}\text { Constant coefficient } \\
\alpha_{0 j}\end{array}$ \\
\hline Peak hand load $(K g)$ & $14.3(12.4)$ & $1.8(1.15-2.87)$ & 0.035 & \multirow{7}{*}{-1.339} \\
\hline Peak shear $N$ & $354(159)$ & $1.9(1.16-3.17)$ & 0.003 & \\
\hline $\begin{array}{c}\text { Cumulative compression } \\
M N s / \text { shift }\end{array}$ & $12.2(0.665)$ & $1.9(1.26-2.93)$ & $8.2 \mathrm{E}-08$ & \\
\hline Job control & $56.5(19.2)$ & $1.9(0.89-4.09)$ & -0.020 & \\
\hline Perceived exertion & $6(1.6)$ & $3.2(1.91-5.70)$ & 0.582 & \\
\hline Social environment & $12.4(1.7)$ & $2.8(1.42-5.84)$ & -0.343 & \\
\hline Over educated level & $($ yes $/$ no $)=1 / 0$ & $2.3(1.09-5.15)$ & 0.833 & \\
\hline
\end{tabular}

Table 6.2: Low back pain WIH risk factor measures and parameters used in the Logistic regression

In a case-control statistical analysis, the in-pain/injured subjects (cases), are usually designated according to a non-randomized sampling method. Hence, Logistic regression needs an adjustment to calculate the correct probability of (proportion) reporting low back pain (King and Zeng 2002). For this reason, as shown in Equation (6.20), the constant coefficient of the

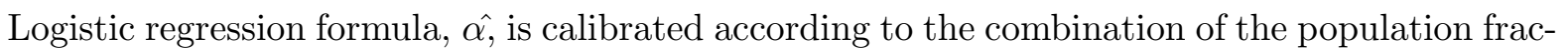
tion of employees reporting low back pain, $\chi$, and the prevalence rate of employees reporting low back pain in the Kerr (1997) study, $y^{\prime}$.

$$
\alpha_{0 j}=\hat{\alpha}-\ln \left(\frac{1-\chi}{\chi} \times \frac{y^{\prime}}{1-y^{\prime}}\right)=-1.2-\ln (1.5 \times 0.766)=-1.339 .
$$

Equation (6.21) adjusts the value of $\Lambda_{j t}$, taking into account statistical estimations derived from the primary data in the Kerr $\mathrm{PhD}$ thesis Kerr (1997) in order to determine the proportion of the in pain state and absent state state operators from the Kerr Logistic regression's outputs.

$$
\Lambda_{j t}=v_{j t}^{w}+v_{a j t}^{a}-\left(v_{j t}^{w} \cap v_{j t}^{a}\right) \quad, \text { where, } \quad \Lambda_{j t}=1.35 v_{j t}^{w} \quad \text { and } \quad \Lambda_{j t}=2.65 v_{j t}^{a}
$$

Finally, Equations (6.4), (6.5), and (6.6) let us complete the generator matrix for each operator of the assembly line in order to determine the steady-state probability of each healthstate.

\section{Step 2: Probability of Making Errors}

According to Equation (6.8), the constant probability of making errors for the healthy and replaced operators is 0.00137 . This probability value is within the acceptable range of the base 
line human errors determined by previous literature (e.g., Bubb 2005).

Similarly, Equation (6.9) determines the average of the probability of making errors for the in-pain state operators as 0.04 , which is also within the acceptable range for the average probability of making errors due to work-related health problems, discussed in previous literature (Yeow and Sen 2006: Ruckart and Burgess 2007). This probability is used for the in-pain operators state when all WIH risk factors are set to their mean exposure levels, described by Kerr (1997). Furthermore, the average excessive fatigue is assumed to be $25 \%$ for the in-pain state operators when all WIH risk factors are set to their mean exposure levels. This assumed fatigue level is consistent with the acceptable fatigue range, that is affecting human performance (e.g, Parhizi and Pasupathy 2013).

Due to changing low back pain WIH risk factor levels, the fatigue level as well as the probability of the "in-pain" operator's error rate vary. The value of $\Theta_{j}^{w}$ is equal to 0.0131 , which is called the "medium workload" in this study, when the average in-pain operator's fatigue level is assumed to be $25 \%$ (all risk factors are at their mean values). By changing the low back pain WIH risk levels, the Markov chain model determines the new probability of being in the "pain" state. Moreover, the operator works under different working condition, which is causing a new workload, $\Theta_{j}^{w}$, value for the fatigue formula. In this study, the new $\Theta_{j}^{w}$ is estimated by multiplying the "medium workload" value with the ratio of the new probability of the "in-pain" state to the probability of being in the "pain" state when all risk factors are at their mean exposure levels. By this estimation, Equation (6.10) determines the new excessive fatigue level.

To track the impact of WIH risk factor changes on the probability of making errors by an operator, it is assumed that the slope parameter of the Weibull distribution proportionally changes with the operator's fatigue level in the pain state. That is, the new value of the slope parameter, $\zeta_{j}^{w}$, is determined according to the ratio of the new fatigue level to the fatigue level of the operator when all risk factors are at their mean exposure levels.

\section{Step 3: Total Cost Minimization}

In this step, the steady-state probability of healthy, pain and absent states and their operational and financial effects are applied into the developed optimization model. Then, the total cost function is minimized using IBM ILOG Cplex 12.1. It is assumed that all operators of the 
sample assembly line have the same risk factor levels. By this assumption, we can vary risk factor exposures within certain levels in order to investigate their effects on the system level performance optimization. Three different scenarios are developed to evaluate the effects of low back pain WIH risk factors.

\subsubsection{Scenario 1: Individual Influence of WIH Risk Factors}

To better understand the individual financial consequences of WIH risk factors, this scenario considers the variation of each low back pain WIH risk factor separately. First, all risk factors are set to their mean values. Then, individually, each risk factor is changed within a distance of \pm 3 standard deviations (std) from its mean value for all operators. The corresponding steady-state probability of each health-state and the associated probability of making errors are determined. Finally, the total cost of the assembly system is minimized according to the new probabilities.

Figures (6.1) and (6.2) portray the behavior of the optimum total cost "index" of the assembly line versus the physical and psychosocial WIH risk factor changes. This cost index is determined at each risk factor exposure level and compared with the cost optimization model without including HF. The total cost model without HF effects is optimized at $\$ 3,387,630$, which is the baseline cost index at 100. The model without HF aspects includes a fixed production rate for each operator, who are assumed to work at their $100 \%$ productivity level. The optimization model also considers the healthy state's probability of making defective products. It does not include indirect OHS and wage loss costs due to ignoring WIH effects.

Figure (6.1 a) demonstrates the total cost index variation due to different cumulative compression loading for all operators. Due to changing the cumulative load intensification from 5.5 to $38.8 \mathrm{MNs} /$ shift, the probability of having pain at work increases from $14.4 \%$ to $39.11 \%$ when all other risk factors are set to their mean levels, described by Table 6.2. Similarly, the chance of being absent changes between $7.74 \%$ and $19.13 \%$, respectively. This load increment increases the associated quality and productivity loss expenses, which are raising the optimum total cost between $1.5 \%$ and $29 \%$, compared with the model without HF effects. There is a similar trend when the model examines the effects of the other physical WIH risk factors, shown in Figures (6.1p and c).

Without including HF effects, it is estimated that the assembly line production quantity 


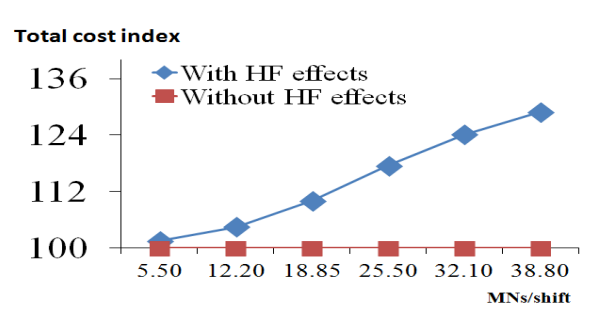

a) The total cost index vs. Cumulative compression changes

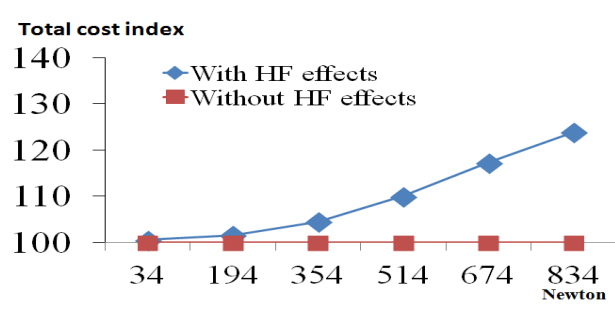

b) The total cost index vs. Peak shear changes

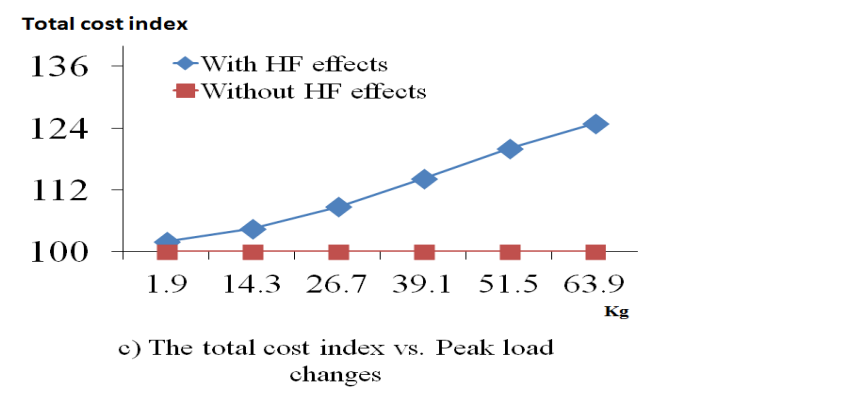

Figure 6.1: The relative total cost index variation versus Physical WIH risk factor changes

will satisfy all annual demand and only 97 products are scrapped annually due to human errors. These rejected products form $0.28 \%$ of the total production. However, due to the negative health-effects of the cumulative compression loading, the percentage of scrap products significantly increases. For instance, with $5.5 \mathrm{MNs} /$ shift compression loading, an operator's has $14.4 \%$ chance of having low back pain at work, which leads to $18.68 \%$ excessive fatigue, accumulated in his/her body. This work-related fatigue raises the expected percentage of defective products to $0.54 \%$ of the production of the assembly line, 185 units. Finally, with 38.8 MNs/shift compression loading level, the "in-pain" state probability reaches to $39.11 \%$, causing the model to generate $42.8 \%$ excessive fatigue. Therefore, 2,153 products are scrapped, which are forming $7.4 \%$ of the assembly line's throughput.

Figure $(6.2$ c) shows the performance effects of integrating job control into the total cost optimization of the assembly line. The total cost declines as job control increases and injury risk reduces correspondingly. At the lowest exposure level, 18.1, the operators psychosocially perceive minimum control over their jobs and minimum contribution in making production decisions. Under these conditions, they have the highest chance of having work-related low back problems. They respectively have $28.9 \%$ and $14.6 \%$ chances of the "in-pain" and "absent" 


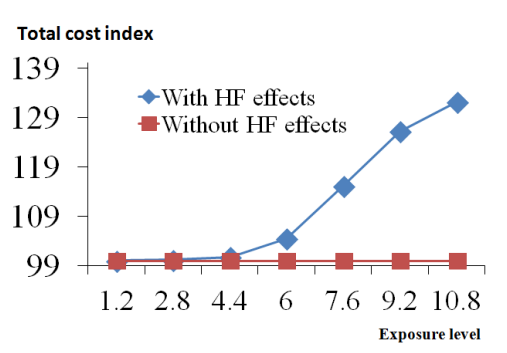

a) The total cost index vs. Perceived exertion changes

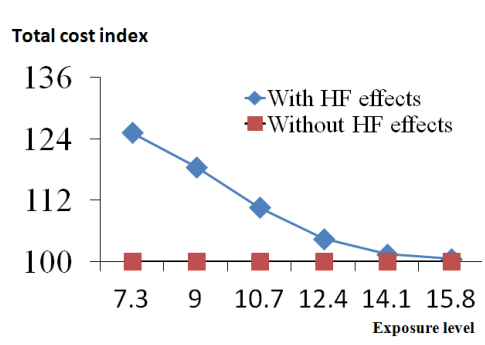

b) The total cost index vs.

Social environment changes

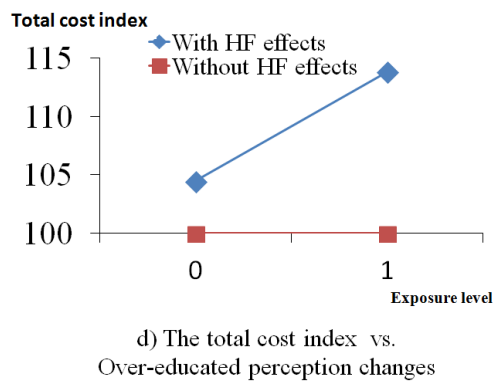

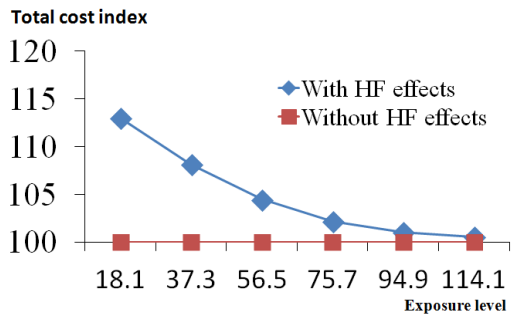

c) The total cost index vs. Job control changes

Figure 6.2: The relative total cost index versus Psychosocial WIH risk factor changes

states, raising the optimum total cost by $13 \%$. Compared to the model without HF effects, this total cost change is mainly due to: a) the productivity loss of the "in pain" operators and new workers causing $\$ 218,130$ subcontracting expenses; and b) the increased human-related probability of making errors for the "in-pain" state, raising the health-related quality deficits cost to $\$ 195,936$. By improving the psychosocial atmosphere of the working environment, the operators perceive a higher control over their assigned tasks. Hence, with 114.1 job control exposure level, the probability of having low back pain decreases to $9.3 \%$ and $5 \%$ for "in-pain" and "absent" states respectively. Consequently, the total cost increases by only $1 \%$. There is a similar behavior in the presence of other psychosocial risk factor variations, shown by figures (6.2 a, $\mathrm{b}$ and d). The mechanism of perceived physical exertion, job control, social support and over-educated in developing MSD were investigated in previous studies (e.g., Carayon et al. 1999; Devereux et al. 1999; MacFarlane and Silman 2000). In general, psychological risk factors can increase muscle tensions which are intensifying physical strain over human body. This increased physical strain subsequently contributes to the appearance of MSD symptoms (e.g., EUOSHA 2008, 2011).

For more details, Table 6.3 demonstrates the variations of the health-state probabilities; 


\begin{tabular}{|c|c|c|c|c|c|c|c|c|c|}
\hline Risk factors & $\begin{array}{c}\text { Exposure level } \\
\text { level }\end{array}$ & $\pi_{j \tau}^{h}$ & $\pi_{j \tau}^{w}$ & $\pi_{j \tau}^{a}$ & $F_{j \tau}^{w}$ & $\begin{array}{l}p_{j t}^{w} \\
(\%)\end{array}$ & $\begin{array}{l}\text { scraping } \\
\operatorname{cost}(\$)\end{array}$ & Total $\operatorname{cost}(\$)$ & Cost per unit $(\$)$ \\
\hline $\begin{array}{c}\text { Peak shear } \\
\text { (newton) }\end{array}$ & $\begin{array}{c}34 \\
194 \\
354 \\
514 \\
674 \\
834 \\
\end{array}$ & $\begin{array}{l}0.85 \\
0.78 \\
0.69 \\
0.61 \\
0.53 \\
0.46\end{array}$ & $\begin{array}{l}0.10 \\
0.15 \\
0.20 \\
0.26 \\
0.31 \\
0.36 \\
\end{array}$ & \begin{tabular}{l|}
0.05 \\
0.08 \\
0.11 \\
0.13 \\
0.16 \\
0.18 \\
\end{tabular} & $\begin{array}{l}0.13 \\
0.19 \\
0.25 \\
0.31 \\
0.36 \\
0.41\end{array}$ & $\begin{array}{c}0.23 \\
1.41 \\
4.02 \\
7.34 \\
10.39 \\
12.70 \\
\end{array}$ & $\begin{array}{c}9,268 \\
17,280 \\
46,018 \\
99,796 \\
169,771 \\
186,637 \\
\end{array}$ & $\begin{array}{l}3,407,460 \\
3,440,560 \\
3,539,250 \\
3,726,380 \\
3,974,250 \\
4,197,620\end{array}$ & $\begin{array}{c}98.81 \\
99.86 \\
103 \\
109.51 \\
119.3 \\
136.92 \\
\end{array}$ \\
\hline $\begin{array}{l}\text { Peak load } \\
\text { (Kg) }\end{array}$ & $\begin{array}{c}1.9 \\
14.3 \\
25.7 \\
39.1 \\
51.5 \\
63.9 \\
\end{array}$ & $\begin{array}{l}0.76 \\
0.69 \\
0.62 \\
0.56 \\
0.50 \\
0.46\end{array}$ & $\begin{array}{l}0.16 \\
0.20 \\
0.25 \\
0.29 \\
0.33 \\
0.36 \\
\end{array}$ & \begin{tabular}{l|}
0.08 \\
0.11 \\
0.13 \\
0.15 \\
0.17 \\
0.18 \\
\end{tabular} & $\begin{array}{l}0.20 \\
0.25 \\
0.30 \\
0.34 \\
0.38 \\
0.40\end{array}$ & $\begin{array}{c}1.8 \\
4.02 \\
6.65 \\
9.2 \\
11.36 \\
13.02\end{array}$ & $\begin{array}{c}21,095 \\
46,018 \\
85,940 \\
142,480 \\
166,207 \\
194,332\end{array}$ & $\begin{array}{l}3,453,930 \\
3,539,250 \\
3,681,360 \\
3,869,260 \\
4,066,830 \\
4,230,900\end{array}$ & $\begin{array}{c}100.26 \\
103 \\
107.93 \\
114.77 \\
128.31 \\
138.76\end{array}$ \\
\hline Job control & $\begin{array}{c}18.5 \\
37.3 \\
56.5 \\
75.7 \\
94.9 \\
114.1\end{array}$ & $\begin{array}{l}0.57 \\
0.63 \\
0.69 \\
0.75 \\
0.81 \\
0.86\end{array}$ & $\begin{array}{l}0.28 \\
0.24 \\
0.20 \\
0.16 \\
0.12 \\
0.09\end{array}$ & $\begin{array}{l}0.15 \\
0.13 \\
0.11 \\
0.09 \\
0.07 \\
0.05\end{array}$ & $\begin{array}{l}0.33 \\
0.29 \\
0.25 \\
0.21 \\
0.16 \\
0.12\end{array}$ & $\begin{array}{l}8.71 \\
6.38 \\
4.02 \\
2.02 \\
0.73 \\
0.16\end{array}$ & $\begin{array}{c}13,0624 \\
81,518 \\
46,018 \\
22,880 \\
12,154 \\
8,983\end{array}$ & $\begin{array}{l}3,828,340 \\
3,663,520 \\
3,539,250 \\
3,460,080 \\
3,421,870 \\
3,405,630\end{array}$ & $\begin{array}{c}113.19 \\
107.25 \\
103 \\
100 \\
99.30 \\
98.75\end{array}$ \\
\hline Perceived exertion & $\begin{array}{c}1.2 \\
2.8 \\
4.4 \\
6 \\
7.6 \\
9.2 \\
10.8\end{array}$ & \begin{tabular}{l|}
0.97 \\
0.92 \\
0.83 \\
0.69 \\
0.55 \\
0.44 \\
0.39
\end{tabular} & $\begin{array}{l}0.02 \\
0.05 \\
0.11 \\
0.20 \\
0.30 \\
0.37 \\
0.41\end{array}$ & $\begin{array}{l}0.01 \\
0.03 \\
0.06 \\
0.11 \\
0.15 \\
0.19 \\
0.20\end{array}$ & $\begin{array}{l}0.03 \\
0.07 \\
0.15 \\
0.25 \\
0.35 \\
0.41 \\
0.45\end{array}$ & $\begin{array}{c}6.08 \mathrm{E}-10 \\
1.33 \mathrm{E}-03 \\
0.42 \\
4.02 \\
9.6 \\
13.48 \\
15.42\end{array}$ & $\begin{array}{c}8,721 \\
8481 \\
10,248 \\
46,019 \\
149,662 \\
178,846 \\
203,973\end{array}$ & $\begin{array}{l}3,391,090 \\
3,395,850 \\
3,413,010 \\
3,539,250 \\
3,902,220 \\
4,276,800 \\
4,477,590\end{array}$ & $\begin{array}{c}98.21 \\
98.37 \\
99 \\
103 \\
116.47 \\
145.66 \\
161.93 \\
\end{array}$ \\
\hline Without HF & - & 1 & - & - & - & 0.137 & 8,897 & $3,387,630$ & 98.11 \\
\hline
\end{tabular}

Table 6.3: Examples of WIH impacts on work-related health, operational and financial aspects

the cumulative fatigue; the associated probability of making errors; the total scraping cost; the minimized total cost; and the per unit production cost versus changing the low back pain risk factor exposure levels.

\subsubsection{Scenario 2: Low Back Pain WIH risk Factor Dual Effects}

This scenario aims to evaluate the effects of two low back pain risk factors at a time, shown by Figures $(6.3 \mathrm{a}$ and $\mathrm{b}) .2 \mathrm{WIH}$ risk factors are simultaneously changed by \pm 3 standard deviations (std) from their mean values for all operators, while the other risk factors are set to their average levels. Then, the new probabilities of the given heath-states; their operational effects; and the probability of making errors are estimated to minimize the total cost of the assembly line. 
Figure $(6.3$ a $)$ demonstrates the total cost index of the assembly system, which is varied due to the dual effects of peak load and cumulative compression loading. In this example peak load is varied between $1.9 \mathrm{~kg}$ and $63.9 \mathrm{~kg}$, while at each exposure measure, the cumulative compression is varied from 5.5 MNs/shift to $38.8 \mathrm{MNs} / \mathrm{shift}$.

In general, the results convey that the optimum total cost at the given peak load level also depends on different compression loading exposures. For example, with $14.3 \mathrm{~kg}$ peak load during working shifts, the probabilities of the "in- pain" and "absent" states changes from, $14.48 \%$ and $7.74 \%$ to $39.11 \%$ and $19.13 \%$, respectively. This work-related dual risk factor effects cause the total cost index to increase between 102 and 128, which is mainly due to: a) changing the annual outsourced number of products from 121 to 6,630 units and; b) increasing the number of scrap products from 186 to 2,153 units. The extra expenses due to the higher peak load and cumulative compression exposures increase the cost per unit of the product from $\$ 99.8$ to $\$ 140$. There are similar trends for all other WIH risk factor joint effects.
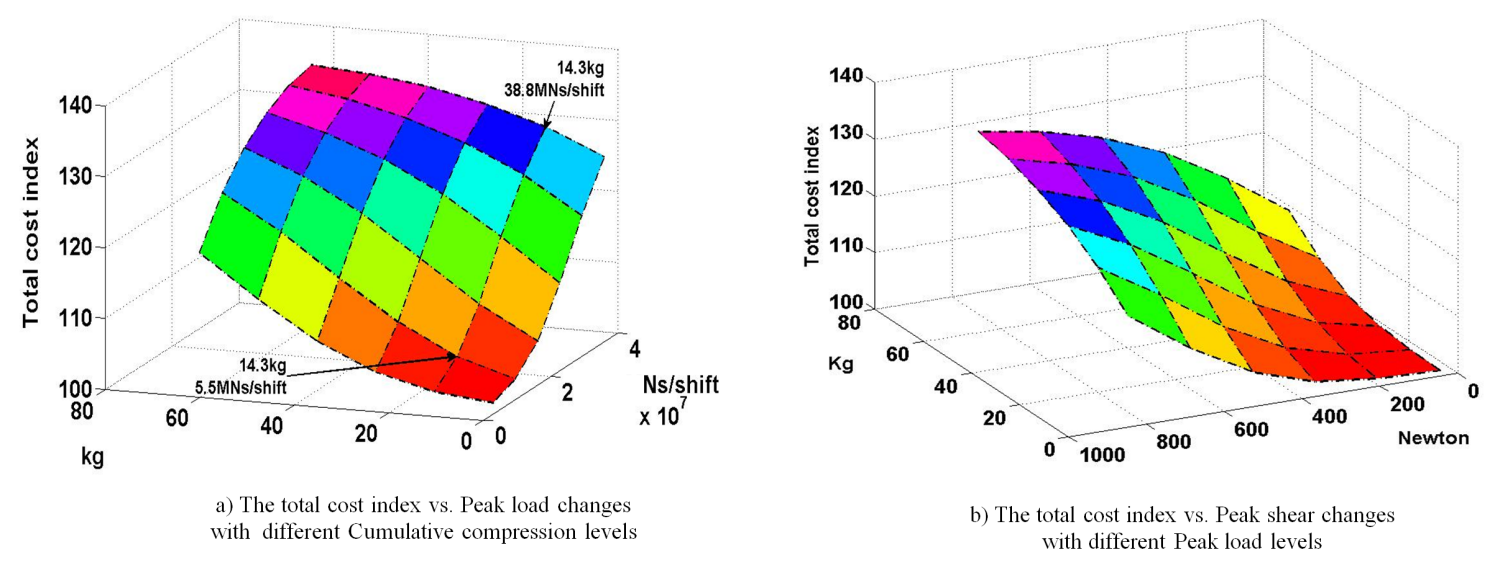

Figure 6.3: The relative total cost index variation versus WIH risk factors dual effects

\subsubsection{Scenario 3: Low Back Pain Odds Ratio Effects}

This scenario aims to evaluate the impact of the strength of the WIH risk factors on developing work-related low back problem and the subsequent financial consequences. As shown in the epidemiology literature, a WIH risk factor may have a different contribution level in developing health problems which is depending on a worker's individual body characteristics, and the ergonomics status of the workplace design (e.g., Kerr et al. 2001; Sim et al. 2006). 
In scenario 3, the effects of the variability in the WIH risk factor odds ratios themselves are investigated. An odds ratio indicates the strength of the association between a given risk factor exposure and the work-related health problem. Generally, a higher odds ratio shows a greater injury risk. Hence, the odds ratio of each risk factor is varied to test the different strength levels of the given risk factor in developing low back problem. Similar to Scenario 1, each risk factor is also changed within a distance of \pm 3 std from its mean measure at a time while the other risk factors are set at their average measures for all operators. Finally, with the given odds ratios, the steady-state probabilities of being in the health-states are calculated for all operators and the total cost model is minimized, shown by Figure (6.4). This test examine the robustness of the developed model according to change the odds ratios.

Figure (6.4 a) shows the influence of peak shear odds ratio variation on the total cost index of the assembly system. A lower odds ratio demonstrates a lower contribution level of the peak shear in developing work-related low back pain. Therefore, compared with a higher odds ratio at a given peak shear exposure level, the probabilities of the in-pain and absent states reduce. For instance, with the $674 \mathrm{~N}$ spine shear force, the probability of having pain at work changes between $13.3 \%$ and $41.1 \%$ when the strength of the shear force in developing low back problem (odds ratio) is changed from 1.16 to 3.17 . Similarly, the probability of the absent state increases from $7.1 \%$ to $20.1 \%$. Consequently, the negative operational and financial effects of peak shear loads increase.

By setting the odds ratio to 1.16, the operators have the minimum low back pain-related productivity loss at work resulting in only 103 products to being outsourced in a year. Furthermore, the expected human-related quality deficit rate is equal to $0.25 \%$ of the production for each operator, causing 152 units to be scraped and rejected from the line annually. By increasing the odds ratio of peak shear force to 3.17 , however, the company is compelled to order 7,926 items to subcontractors and satisfy the annual demand. The higher odds ratio has also increased the cumulative fatigue level of the operators, which raises the expected probability of producing defective items to $6.57 \%$. Hence, 2,270 products are scrapped in a year. All these HF-related consequences lead the total cost of the assembly line to be optimized at a higher amount. The total cost index increases from 101.12 to 132.6. A similar trend happens by varying the contribution levels of the other physical and psychosocial risk factors, shown in Figures 6.4 b, c, d, e and f). 

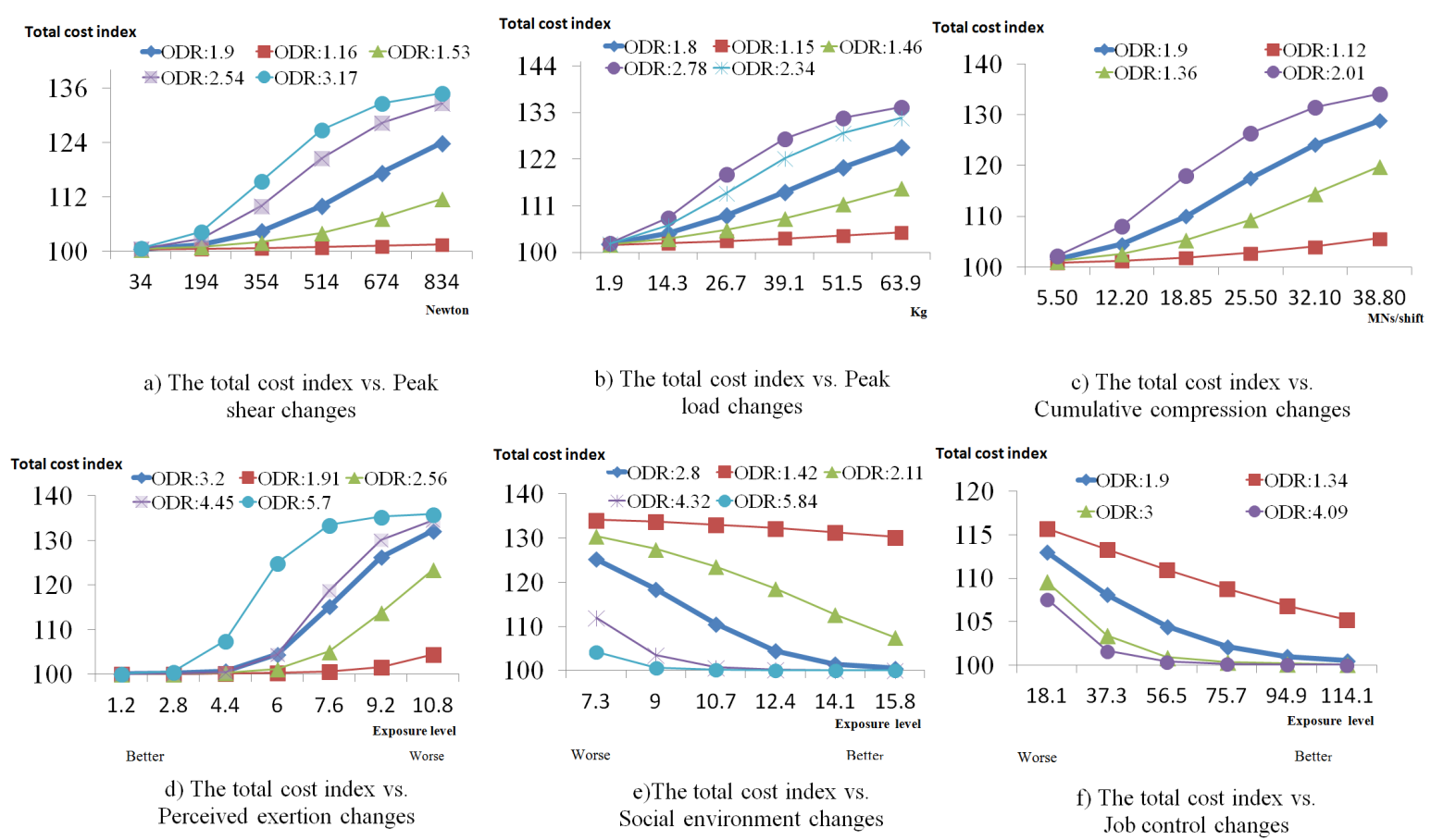

Figure 6.4: The behavior of the total cost index of the assembly system versus low back pain WIH odds ratio (ODR) changes

\subsubsection{Details on Health-related Productivity Loss Variation}

The relationship between work-related health problems and human performance reduction at work has been addressed in previous studies (e.g., Heuvel et al. 2010). Medical and epidemiology literature demonstrates different valid tools designed to measure work-related health problems (Meerding et al. 2005; Burton et al. 2005). There is a wide range of variability to measure MSD-related productivity loss at work. Furthermore, the results of the measurement tools are different from one industry to another. In the above numerical analysis, the author applied $16.6 \%$ health-related productivity loss. In order to evaluate the robustness of the developed model against health-related productivity loss variation, the optimization model is tested in this section by changing the assembly line operators' on-job productivity loss at two levels of $6 \%$, and $26 \%$ below and above the average productivity loss, used in previous numerical analysis. As an example, Table 6.4 demonstrates the results of different health-related productivity losses at work versus peak load and perceived exertion changes.

When all risk factors are set to their mean exposure levels, the cost per unit of the final 


\begin{tabular}{|c|c|c|c|c|c|c|c|c|c|}
\hline \multirow{3}{*}{ Risk factors } & \multirow{3}{*}{$\begin{array}{l}\text { Exposure } \\
\text { level }\end{array}$} & \multicolumn{4}{|c|}{$6 \%$ productivity loss } & \multicolumn{4}{|c|}{$26 \%$ productivity loss } \\
\hline & & \multicolumn{8}{|c|}{ Assembly system cost items } \\
\hline & & $\begin{array}{c}\text { Total cost } \\
(\$)\end{array}$ & Cost index & $\begin{array}{l}\text { Subcontract } \\
\text { (cost) }\end{array}$ & $\begin{array}{l}\text { Cost per } \\
\text { unit }(\$)\end{array}$ & $\begin{array}{c}\text { Total cost } \\
(\$)\end{array}$ & Cost index & $\begin{array}{c}\text { Subcontract } \\
\text { (units) }\end{array}$ & $\begin{array}{l}\text { Cost per } \\
\text { unit }(\$)\end{array}$ \\
\hline \multirow{6}{*}{$\begin{array}{l}\text { Peak load } \\
\quad(\mathrm{kg})\end{array}$} & 1.9 & $3,438,550$ & 101.50 & 6,475 & 99.5 & $3,470,200$ & 102.44 & 50,050 & 101.16 \\
\hline & 14.3 & $3,520,040$ & 103.91 & 26,075 & 102 & $3,559,600$ & 105.08 & $1,090,250$ & 104.43 \\
\hline & 25.7 & $3,658,390$ & 107.99 & 78,050 & 106.03 & $3,705,100$ & 109.37 & 211,575 & 110 \\
\hline & 39.1 & $3,844,830$ & 113.50 & 165,725 & 112.07 & $3,892,980$ & 114.92 & 359,975 & 117.66 \\
\hline & 51.5 & $4,046,100$ & 119.44 & 504,875 & 124.45 & $4,085,780$ & 120.61 & 753,200 & 132.17 \\
\hline & 63.9 & $4,217,430$ & 124.50 & 730,625 & 134.45 & $4,242,870$ & 125.26 & 986,825 & 142.93 \\
\hline \multirow{7}{*}{$\begin{array}{l}\text { Perceived } \\
\text { exertion }\end{array}$} & 1.2 & $3,389,400$ & 100.05 & 0 & 98.16 & $3,392,500$ & 100.14 & 0 & 98.25 \\
\hline & 2.8 & $3,391,260$ & 100.11 & 350 & 98.23 & $3,400,190$ & 100.37 & 350 & 98.54 \\
\hline & 4.4 & $3,401,890$ & 100.42 & 1,925 & 98.53 & $3,424,110$ & 101.08 & 22,925 & 99.53 \\
\hline & 6 & $3,520,040$ & 103.91 & 26,075 & 101.75 & $3,559,600$ & 105.08 & 109,025 & 104.43 \\
\hline & 7.6 & $3,877,730$ & 114.47 & 207,550 & 113.69 & $3,925,520$ & 115.88 & 406,700 & 119.55 \\
\hline & 9.2 & $4,265,400$ & 125.91 & 926,975 & 141.39 & $4,286,610$ & 126.54 & $1,154,830$ & 149.55 \\
\hline & 10.8 & $4,475,380$ & 132.11 & $1,251,950$ & 157.44 & $4,479,670$ & 132.24 & $1,468,250$ & 166.14 \\
\hline
\end{tabular}

Table 6.4: Impacts of productivity loss variation on the assembly line optimum cost items

product is equal to $\$ 102$ with $6 \%$ productivity loss for the in-pain operators. It shows $0.97 \%$ reduction from the associated cost with $16.6 \%$ productivity loss. With $26 \%$ productivity loss, the cost per unit of the product is equal to $\$ 104.4$, demonstrating $1.36 \%$ growth, compared to the value of the cost per final product with $16.6 \%$ productivity loss. Compared to the model without including HF effects, the operational and financial effects of HF still show significant difference for the optimized total cost of the assembly line when the productivity loss is changed from $6 \%$ to $26 \%$. For instance, at the peak load mean value, $14.3 \mathrm{~kg}$, the total cost shows between $3.9 \%$ and $5.1 \%$ growth, when productivity loss changes from $6 \%$ to $26 \%$. A similar trend is found in perceived exertion analysis.

\subsection{Managerial Advantage of The Model}

A systematic modeling connection between the workplace HF conditions and the performance of operation systems is the main advantage of the developed model. This modeling approach assists managers to better understand how the ergonomic design of the workplace affects the behavior of the operations system. It also enables the model's users to track financial, operational and health effects of WIH risk factor changes at the system level. Managers also gain a clear 
view about the expected financial benefits of implementing ergonomic interventions, which are reducing WIH risk factor negative cost effects. Hence, they will achieve a better estimation of cost-benefit analysis for different ergonomic design solutions. As an example, Figure 6.5 shows the financial, operational and health effects of cumulative compression changes based on the scenario 1's experimental conditions.

In terms of the system level financial effects, Figure $(6.5$ a) shows that the behavior of the minimum total cost of the assembly system due to different ergonomic designs of the workstations. The hypothetical workplace HF design causes the cumulative compression load to vary between 5.5 to $38.8 \mathrm{MNs} /$ shift. Consequently, the optimum total cost of the system varies between $\$ 3,440,000$ and $\$ 4,366,480$, respectively (27\% growth).

At 38.8 MNs/shift, the operators are exposed to the highest level of accumulated compressive force on their low back joints. This higher level cumulative force may be caused by : a) a higher number of task repetitions during working-shifts; b) a longer time that the operators are exposed to physical exertion in accomplishing assembly tasks; or c) a higher external manual handling load level that the operators are exposed to when they carry objects during working hours. As a result, the chance of having work-related low back problem becomes maximum. It is $39.1 \%$ for the in-pain state and $19.33 \%$ for the absent state. By improving ergonomic conditions of the work such as using a conveyer or a lift-assist to reduce manual handling load, the chance of work-related health problem is reduced as well. At $5.5 \mathrm{MNs} / \mathrm{shift}$, the health-state probabilities become $14.4 \%$ and $7.7 \%$ for in-pain and absent states, respectively. Ultimately, using the developed model supports managers to better estimate and compare financial and occupational health and safety consequences of different ergonomic solutions for improving physical design of the workplace.

The insurance premium is one of the important OHS cost items, which is showing the financial contribution of occupational health problems at the system level (e.g., Riel and Imbeau 1998). The results of the model in this study demonstrate a significant relation between the insurance cost and WIH risk factor changes (ergonomic design of the workplace).

The models without HF effects are unable to consider the effects of this type of relation at the system level performance analysis. As shown by Figure (6.5), at the lowest cumulative compression level, the insurance premium for all 4 operators is equal to $\$ 3,722$. However, a poor ergonomic design of the system increases medical treatment and compensation benefit 


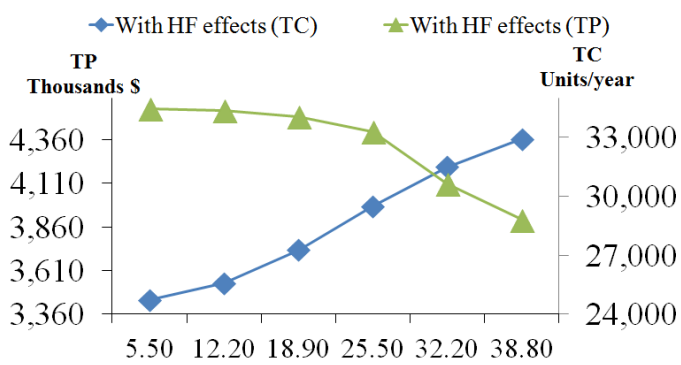

MNs/shift

a) Total production (TP) and Total cost (TC) vs. Cumulative compression changes

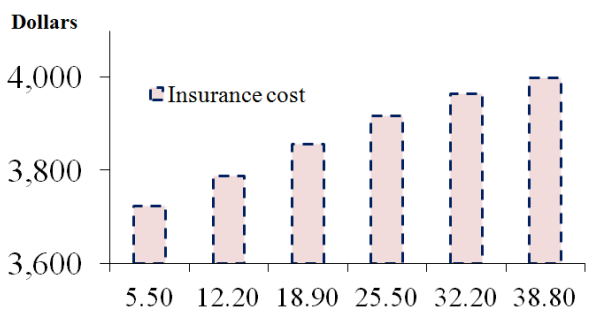

MNs/shift

c) Insurance cost vs. Cumulative compression changes

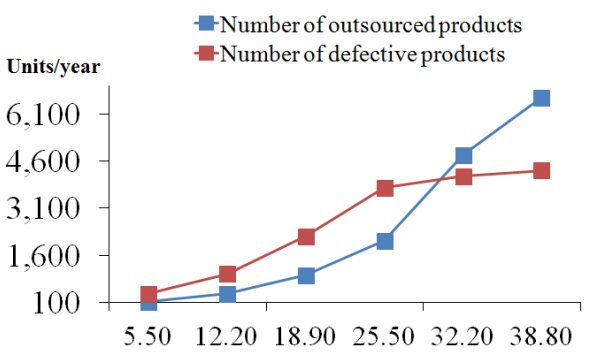

MNs/shift

b) Number of subcontracted and defective products vs. Cumulative compression changes

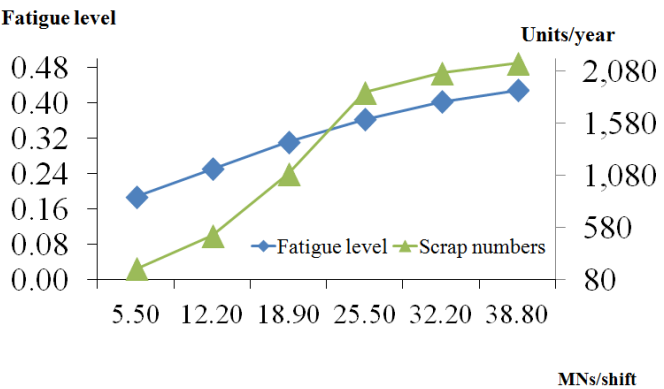

d) Fatigue level and Scrap products vs. Cumulative compression changes

Figure 6.5: Financial, Operational and Health-related effects of a given WIH risk factor

costs which are claimed by in-pain or absent workers due to work-related low back pain. At 38.8 MNs/shift, the operators have the highest chance of being in the "in-pain" and "absent" states. Therefore, the insurance premium raises to $\$ 3,999$. Compared with the average annual insurance premium paid by manufacturing companies in Ontario, Canada, $\$ 3,600$, the determined insurance cost increases between $3.38 \%$ and $11.08 \%$ due to ergonomic design of the serial system. This insurance premium growth incurs huge costs for medium and large manufacturing companies, which insure hundreds of employees in a year (e.g., WSIB 2010a).

In terms of the system level operational effects, including HF aspects in optimizing the performance of the operation system enables the model's users to analyze throughput, defective and outsourced product quantities versus changing ergonomic design of the system, shown by Figures $6.5 \mathrm{a}$ and b). In general, the interpretation of these HF-related consequences 
assist managers to predict effective production planning and to build a strategy for outsourcing production. For instance, changing the ergonomic design of the assembly workstations results that the cumulative compression exposure level varies from $5.5 \mathrm{MNs} / \mathrm{shift}$ to $38.8 \mathrm{MNs} / \mathrm{shift}$. Subsequently, integrating operational and financial effects of this ergonomic changes into the optimization model causing the optimum production of the line varies between 34,452 and 28,806 units, respectively. This reduction is mainly due to work-related productivity and quality losses of the employees. The optimization model without including HF effects estimates 34,528 units, produced by the assembly system. Compared to the model with HF effects, the production quantity is overestimated between $0.2 \%$ and $19.9 \%$ due to the poor ergonomic design of the workplace. Failure to include these operational effects, prevents managers from addressing and examining the production variations due to poor ergonomic design, which leads to inaccurate production planning. Therefore, the company may have back orders and lose its customers.

This research also demonstrates an increasing dependency on subcontractors by outsourcing more products, which is forcing the company to make a business collaboration with the subcontractors. For instance, as shown by Figure (6.5b), at the lowest compression level, 185 defect products are rejected from the assembly line occurs due to human errors. Also, 121 units are ordered to be provided by subcontractors in a year. However, with $38.8 \mathrm{MNs} / \mathrm{shift}$ compression exposure, the operators' error rates increase, causing 2,153 defective products to be scrapped an rejected from the assembly system. Moreover, the work-related operators' performance loss obliges the assembly system's managers to order 6,630 units to outsource companies (subcontractors) to satisfy customer demand. This increased HF-related dependency on the subcontractors enlarges the supply chain network of the company that can influence on the time of delivery, production expenses and the quality of production. Therefore, without establishing a high integrative collaboration among supply chain partners (which requires considerable investment), the delivery time of product to customers as well as the final price and the quality of the product would be negatively affected which may reduce customer satisfaction and market share. By improving the HF design of the workplace, the management of the supply chain network becomes more efficient since fewer third parties (subcontractors) are involved in producing the final product. Hence, managers can better establish a long term production plan for the company while the dependency on the subcontractors is reduced and the uncertainties involved in providing final products would decline. 
In terms of investigating work-related health effects, the model allows to understand the effects of workload on human fatigue, injury risks and quality performance of operators. For instance, Figure 6.5 d) demonstrates that a better ergonomic design of the assembly system reduces compression loading over the lower back joints of the operators from 38.8 to $5.5 \mathrm{MNs} / \mathrm{shift}$. This workload reduction causes less excessive fatigue to be developed in the operator's body. Hence, the chance of having low back pain subsequently reduces. Consequently, defective items (scrapped and reworked items) produced due to human errors in the manufacturing system reduces from 4,306 to 370 units.

Apart from the fatigue-related human error making, working with a high cumulative fatigue level also affects the quality of the life of the operators (Mock et al. 2001). Hence, they may not be able to completely accomplish their after work (social and family) responsibilities. By continuing this condition, the operators may lose their motivation to keep the highest commitment to the company goals. The lack of commitment due to the work-related health problems may prevent the company from building an effective and strong customer relationship. Therefore, the company may distract from its business goals and lose customer satisfaction (e.g., Nishii et al. 2008). By integrating WIH effects into the system level performance evaluation models, managers may prevent this health-related business malfunction by reducing WIH effects through a better ergonomic design of the workplace.

\subsection{Conclusion}

This research develops a modeling approach to investigate the effects of integrating HF aspects in optimizing the cost of an operation system. Compared with the optimization model without including HF, the numerical analysis has demonstrated a substantial difference in the minimized total cost of the assembly system when WIH risk factor effects are considered. The results show that ignoring WIH effects underestimate the total cost. Without including HF effects, the total cost was minimized at $\$ 3,387,630$. However, this cost grows between $0.26 \%$ and $32 \%$ in the presence of different physical and psychosocial low back pain risk factors. The operators' healthrelated productivity and work quality losses are the major reasons for these cost increments at the system level by: a) reducing the system's throughput; b) producing the substantial number of scrap and rework units; and c) outsourcing the considerable number of products to 
satisfy customer demand on time. These HF-related underestimations are usually ignored in the existing optimization model which may result in inaccurate performance prediction for the company. For instance, if managers estimate 35\% annual net profit for the assembly system, considered in this study, the financial and operational consequences of poor ergonomic design the workplace reduces this profit between $0.74 \%$ and $91.4 \%$ in the presence of different low back pain risk factor exposure levels.

The model developed in this chapter is limited to consider the employees' work-related performance variations due to low back pain. As a general modeling approach, the model's users are able to consider other work-related health problem effects such as neck pain shoulder injuries which can comprise system performance. Furthermore, the proposed model in this chapter focuses on deterministic demand when optimizing the cost of the assembly system. In future it can be extended by evaluating the stochastic behavior of the demand to better replicate the system performance in a more natural way. 



\section{Chapter 7}

\section{Evaluating the performance of a}

\section{serial system with regard to}

\section{stochastic demand and employee work-related performance variation}

The uncertainties in internal resources such as workforce (employee) performance variation and external (market) variables such as stochastic demand can significantly affect operation systems (Desai et al. 2007; Panda et al. 2008; Gua et al. 2010; Dul et al. 2012). Modeling these internal and external uncertainties support system performance evaluation models to replicate the real behavior of the systems.

Market conditions cause customer demand to follow a stochastic behavior rather than deterministic. To investigate the effects of this uncertain behavior, the demand fluctuation is usually modeled within the product life cycle (e.g., Aitkena et al.2003; Fontes 2008, Qin and Nembhard 2012). The pattern of the demand dynamics can be described in terms of the "regime-switching" model for the product life cycle (e.g., Chen and Forsyth 2010). The demand for a new product usually begins with the growth regime, showing an increasing behavior of the demand. Then, it can change stochastically to the decay regime, when the demand of the product decreases (e.g., Bollen 1999). To offset the demand dynamics effects, operation analysts usually consider flexible inventory levels. This approach would increase the ability of the operations system in 
response to the demand from its dynamic environment (Bhattacharye 2005). Moreover, considering different inventory levels enables operation managers to track the impacts of a shift from one level to another on estimating the financial performance of the system throughout the product life cycle (e.g., Bollen 1999). There are many applications of regime-switching models in the Operations Management (OM) literature. For instance, Bollen (1999) developed a real option valuation model to incorporate a stochastic product life cycle. He applied the growth-decay regime-switching approach to value the profit of an operations system, taking into account different capacity levels. Wahab et al. (2010) developed a three regime-switching model determining the valuation of swing options by investigating the daily average price of the electricity. Bollen et al. (2000) used regime-switching models to capture the dynamics of foreign exchange rates. Elias et al. (2014) applied a regime-switching approach investigating the behavior of temperature dynamics behavior with an aim to the valuation of weather option price. To model stochastic variables, lattice is one of the methods of modeling the multi-period of regime-switching variables (e.g., Most and Keles 2010). It is a discretizing approach aiming to achieve computational efficiency in dynamic programming. The lattice method was first proposed by Cox et al. (1979). Huisman and Mahieu (2003) applied lattice method in order to propose a three-state regime-switching model evaluating the stochastic pattern of the spot price in the Nordic power exchange. Wahab and Lee (2009) generalized the lattice method for multiple regime-switching variables with multiple regimes.

However, a literature review in OM system performance evaluation models demonstrates that research studies infrequently consider the health-related performance variation of humans (e.g., Neumann and Dul 2010). Particularly, when human aspects are included in optimization models, scholars usually consider the availability of workers in the system while they assign a fixed value for the performance of the workers who are at work (e.g., Boudreau et al. 2003; Leung and Chan 2009; Chiu et al. 2012, Mirzapour Al-e hashema et al. 2011). These models neither include employee performance fluctuation due to occupational health problems nor evaluate work-related causes and consequences of the occupational health problems at the system level performance analysis.

This study aims to integrate the effects of human's work-related performance variation and stochastic demand behavior into the valuation of the optimum total cost of a serial manufacturing system within the product life cycle. In this chapter, a three-state Markov chain is 
developed for each operator of the system which is modeled his/her work-related health-states. The probability of each state varies taking into account different Work-related Ill Health (WIH) risk factor exposure levels. Furthermore, the demand's pattern in this study follows a two regime-switching approach. To model these regimes, a pentanomial lattice method is used. The total cost of the assembly system is minimized at each node of the lattice with respect to the Human Factors (HF)-related employee performance variations and Occupational Health and Safety (OHS) expenses. The buffers of the assembly line include three specified inventory levels to manage the variability of the demand at each point of the time. The expected total cost of the system is quantified according to different levels of the system's inventories.

The remainder of this chapter is organized as follows. Section 7.1 introduces the features of the serial manufacturing system considered in modeling analysis. Section 7.2 describes the lattice model used to replicate the stochastic behavior of the demand. Section 7.3 describes human health-states Markov chain. Section 7.4 introduces a modeling approach to consider the human-related productivity loss and quality deficits. Section 7.5 introduces a developed optimization model including WIH risk factor effects. Section 7.6 describes backward dynamic programming, applied to the valuation of the optimum total cost with regard to stochastic demand. Section 7.7 examines the robustness of the developed model in terms of WIH risk factors and demand parameter changes. Section 7.8 includes the limitation of the model and conclusion.

\subsection{Serial Production System}

In this section, a serial assembly system is introduced to investigate the effects of employee health variations as well as demand fluctuation on the performance of the system. This production structure is widely used in OM performance optimization studies (e.g., Shang 2012). The structure was introduced in previous chapters. For the readers' convenience, the operational features of the assembly system are briefly reviewed.

The assembly system includes 4 workstations with 4 operators. There is a buffer (inventory) between each two stations. The production starts from the first station and the incomplete product is gradually transferred from one station to another one. The final assembled product is shipped to the customer from the last inventory to satisfy the demand. Production includes 
both regular and over-time shifts. The demand must be fully satisfied during a given time period. So, if the output of the production system can not meet the demand, it is assumed that subcontractors will cover unmet customer orders during the given time period. This study focuses on health-related employee performance variations. Therefore, it is assumed that the assembly machines work perfectly and the production rate of each station is dominated by the corresponding operator's performance. Furthermore, each station may produce defective items due to human errors. Hence, for a given time period, the output of each workstation is inspected individually causing scrapped products to be rejected from the assembly system while reworked products are returned to the station.

To describe the modeling details, first, a lattice based demand estimation is introduced to model the stochastic behavior of the demand within a product life cycle. Then, the effects of WIH risk factors on the operators' health conditions and their performance levels are investigated. Finally, the cost optimization model of the assembly system is explained and dynamic programming is used to estimate the present optimum total cost of the assembly system. The robustness of the developed modeling method is numerically tested at the end of this study.

\subsection{Demand Value Estimation Using Lattice Method}

The lattice is a modeling method for stochastic variables aiming to, first, 1) divide the product life cycle into limited "time-steps" and, then, 2) determine the possible values of the stochastic variable (demand) at each "time-step" which are represented by discrete points (nodes). This discretizing approach lets the lattice users trace the possible paths of growing and falling of the stochastic variable (demand) from one time-step to another one, shown by Figure (7.1).

The developed lattice in this study models the stochastic behavior of the demand by generating the demand values and their associated probabilities within the assembly line's product life cycle. The following assumptions are applied for the stochastic analysis in this chapter: a) the assembly line produces one product to satisfy the customer demand. b) the buffer (inventory) level of each workstation is varied according to the three defined capacity levels. c) the demand includes a 2-regime product life cycle: growth and decay. Each regime is modeled by a geometric Brownian motion. d) the demand can follow the growth or the decay regime at the beginning of the production (life cycle). If it is in the growth regime, it can switch 
stochastically to the decay regime. e) the probability of switching from the growth to the decay regime includes a cumulative normal distribution function over the product life cycle. This time-dependent switching probability is started since the beginning of the production. f) the product life cycle of the assembly system is equal to five years and the size of a "time-step" in the stochastic analysis is equal to one month.

A pentanomial lattice is developed to model the growth and decay regimes through the product life cycle. The growth regime is represented by increasing demand, and the decay regime is represented by decreasing demand. Creating five branches for each node of the pentanomial lattice lets the users to match the means and variances of the growth and decay regimes at each node (Wahab and Lee 2009). Figure (7.1) illustrates a single "time-step" of the pentanomial lattice. $q$ represents the level of the demand starting with the value of 1 . The branches with nodes $(q, \Delta \tau),(q+2, \Delta \tau)$ and $(q+4, \Delta \tau)$ represent the growth regime. The branches with nodes $(q+1, \Delta \tau),(q+2, \Delta \tau)$, and $(q+3, \Delta \tau)$ represent the decay regime. Theoretically, the branch which is including the common node of the growth and decay regimes, $(q+2, \Delta \tau)$, lets the lattice users equalize the step-size (the space between each two branches) of all branches. This equalizing procedure is allowing an efficient recombination of the branches throughout the product life cycle (e.g, Wahab and Lee 2009). This common branch also maintains a match between the original lattice and the distribution implied by the pentanomial lattice (e.g., Bollen 1999). The process of generating nodes (demand values) continues within the product life cycle. The developed pentanomial lattice results in generating $4 \tau-3$ nodes at each time-step.

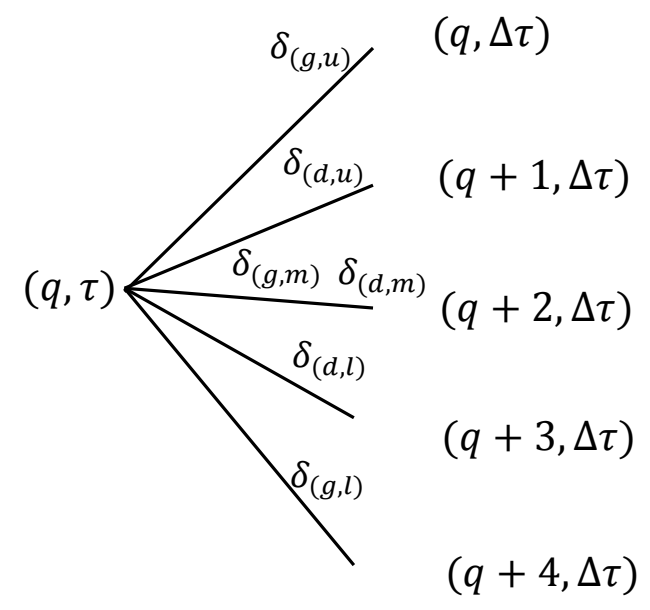

Figure 7.1: The first single time-step of the pentanomial lattice 
The probabilities of the upper, middle, and lower branches for the growth regime are defined as $\delta_{(g, u)}, \delta_{(g, m)}$, and $\delta_{(g, l)}$, respectively. $\delta_{(d, u)}, \delta_{(d, m)}$, and $\delta_{(d, l)}$, are the probabilities of the decay regime's upper, middle, and lower branches. To determine the branch probabilities of each regime, it is necessary to calculate the step-sizes of the regimes. Equation (7.1) shows the step-size of the regime $\psi$, taking into account the associated mean, $\xi_{\psi}$, and volatility, $\sigma_{\psi}$.

$$
\widehat{\theta}_{\psi}=\sqrt{\sigma_{\psi}^{2} \Delta \tau+\left(\xi_{\psi} \Delta \tau\right)^{2}}
$$

$\Delta \tau$ represents the interval time between the 2 consecutive lattice layers. To equalize the space between the five branches of the pentanomial lattice, the step-size of each regime must be calibrated in a way that there is a 1:2 ratio for the step-sizes of the growth-decay lattice model (e.g., Elias et al. 2014). For this reason, $\widehat{\theta}_{\psi}$ values are arranged and re-indexed such that $\theta_{1}<$ $\theta_{2}$. As it is obvious, $\theta_{1} \neq \widehat{\theta}_{1}$. $\theta_{1}$ may include the growth or decay regimes but it is the smallest step-size. Subsequently, we define $\theta=\max \left(\theta_{1}, \frac{\theta_{2}}{2}\right)$. So that, $\theta=\frac{\theta_{\psi}}{\psi}$ and $\frac{\theta_{\psi}}{\psi} \geq \frac{\theta_{\hat{\psi}}}{\hat{\psi}}$ when $\psi$ is not equal with $\hat{\psi}$. Finally, the adjusted step-size of each regime is determined according to the following comparison:

$$
\theta_{\hat{\psi}}= \begin{cases}\theta_{\psi} & \text { if } \psi=\hat{\psi} \\ \hat{\psi} \frac{\theta_{\psi}}{\psi} & \text { if } \psi \neq \hat{\psi}\end{cases}
$$

The conditional branch probabilities of the growth and decay regimes are calculated as the following:

$$
\begin{gathered}
\delta_{\left(\theta_{\hat{\psi}}, u\right)}=\frac{e^{\xi_{\hat{\psi}} \Delta \tau}-e^{\left(-\hat{\psi} \frac{\theta_{\psi}}{\psi}\right)}-\delta_{\left(\theta_{\hat{\psi}}, m\right)}\left(1-e^{\left(-\hat{\psi} \frac{\theta_{\psi}}{\psi}\right)}\right)}{e^{\left(\hat{\psi} \frac{\theta_{\psi}}{\psi}\right)}-e^{\left(-\hat{\psi} \frac{\theta_{\psi}}{\psi}\right)}}, \\
\delta_{\left(\theta_{\hat{\psi}}, m\right)}=1-\frac{\theta_{\hat{\psi}}^{2}}{\left(\hat{\psi} \frac{\theta_{\psi}}{\psi}\right)^{2}}, \\
\delta_{\left(\theta_{\hat{\psi}}, d\right)}=1-\delta_{\left(\theta_{\hat{\psi}}, m\right)}-\delta_{\left(\theta_{\hat{\psi}}, u\right)},
\end{gathered}
$$

Now, a modeling approach is introduced in the next sections to minimize the total cost of the system at time $\tau$, taking into the account the operators' HF-related performance variations, the demand's value at each pentanomial lattice node and the buffer levels of the workstations. 


\subsection{Markov Chain Health-States}

To govern the HF-related performance variation of the operators, this section introduces an approach aiming to link the exposure levels of WIH risk factors with an operator's health-state using Markov chain elements. Among various occupational health problems, this study focuses on Musculoskeletal disorders (MSD) as a common and pervasive work-related injury/illness in developed countries (Matsudaira et al. 2011). It is assumed that an operator in the assembly line have the probabilities of being in three health-states due to negative effects of the workplace HF aspects (WIH risk factors): the healthy without any MSD symptom; the in-pain state at work; or the absent state. A new worker is replaced when an operator is in the absent state. These work-related health-states conform with the output of health measurement tools, used in epidemiological analysis to determine human health conditions (e.g., Dawson et al. 2009).

Markov modeling is a common approach in analyzing human health-related issues (e.g.,

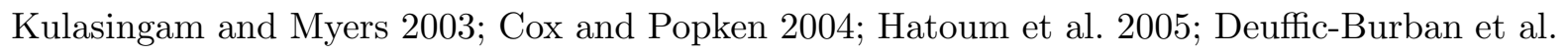
2007; Bowman et al. 2010). To develop the health-state Markov chain of the operator, we focus on the continuous Markov chain concept and Logistic-based Markov approach. For this reason, we construct the generator matrix rate, Equation (7.5), according to different WIH risk factor levels to estimate the stationary probability of each health-state, shown by Equation (7.6).

$$
\begin{gathered}
Q=\left[\begin{array}{ccc}
-\left(\gamma_{j \tau}^{h w}+\gamma_{j \tau}^{h a}\right) & \gamma_{j \tau}^{h w} & \gamma_{\tau}^{h a} \\
\gamma_{j \tau}^{w h} & -\left(\gamma_{j \tau}^{w h}+\gamma_{j \tau}^{w a}\right) & \gamma_{j \tau}^{w a} \\
\gamma_{a h j \tau}^{a h} & \gamma_{j \tau}^{a w} & -\left(\gamma_{j \tau}^{a h}+\gamma_{j \tau}^{a w}\right)
\end{array}\right] \\
Q\left[\begin{array}{l}
\pi_{j \tau}^{h} \\
\pi_{j \tau}^{w} \\
\pi_{j \tau}^{a}
\end{array}\right]=\left[\begin{array}{l}
0 \\
0 \\
0
\end{array}\right] \quad \text { and, } \\
\end{gathered}
$$

The multiple Logistic regression, which is widely used in epidemiology studies, is applied in this research to make a connection between WIH risk factors and the operator's health-states. In the presence of the given WIH risk factor exposure levels, Logistic regression estimates an underlying population fraction which is including individuals who are having work-related health problems (Kleinbaum and Klein 2010). Equation (7.7) demonstrates the Logistic function formula determining the proportion of underlying population, entering to the "pain" and "absent" 
states from the healthy state based on physical and psychosocial WIH risk factor exposures.

$$
v_{j t}^{\bar{h}}=\frac{\exp \left[\sum_{y=1}^{n} \beta_{y j} X_{y j}+\alpha_{0 j}\right]}{1+\exp \left[\sum_{y=1}^{n} \beta_{y j} X_{y j}+\alpha_{0 j}\right]} \quad \text { where, } \quad \bar{h}=\{w, a\}
$$

To estimate the associated rates of entering into the pain and absent states, the outputs of Equation (7.7) are converted to cumulative rates according to Equation (7.8). These rates are subject to change in the presence of WIH risk factor exposure variations.

$$
\lambda_{j t}^{\bar{h}}=\ln \left(\frac{1}{1-v_{j t}^{\bar{h}}}\right) \quad \text { where }, \quad \bar{h}=\{w, a\}
$$

The generator matrix rate is completed by approximating the rate of entering into the pain state from the healthy state. To do so, the transition probabilities from the healthy to the pain and the pain to the healthy state, developed according to continuous and Logistic based Markov analysis, are equalized (Azzalini 1994), shown by Equations (7.9) and 7.10). A similar practice is employed to determine the rate of entering into the absent state from the healthy state.

$$
\begin{aligned}
& \frac{\lambda_{j t}^{\bar{h}}}{\lambda_{j t}^{\bar{h}}+\mu_{j t}^{\bar{h}}}-\frac{\lambda_{j t}^{\bar{h}}}{\lambda_{j t}^{\bar{h}}+\mu_{j t}^{\bar{h}}} \exp \left[-\left(\lambda_{j t}^{\bar{h}}+\mu_{j t}^{\bar{h}}\right) t\right]=v_{j t}^{\bar{h}}\left(1-u_{j t}^{\bar{h}}\right) \quad \text { where, } \quad \bar{h}=\{w, a\} . \\
& \frac{\mu_{j t}^{\bar{h}}}{\lambda_{j t}^{\bar{h}}+\mu_{j t}^{\bar{h}}}-\frac{\mu_{j t}^{\bar{h}}}{\lambda_{j t}^{\bar{h}}+\mu_{j t}^{\bar{h}}} \exp \left[-\left(\lambda_{j t}^{\bar{h}}+\mu_{j t}^{\bar{h}}\right) t\right]=\left(1-v_{j t}^{\bar{h}}\right)\left(1-u_{j t}^{\bar{h}}\right) \quad \text { where, } \quad \bar{h}=\{w, a\} .
\end{aligned}
$$

For this research, the transition rate from the pain state to the absent state and from the absent state to the pain state are estimated by taking the average value of the underlying rates, determined in previous HF studies (e.g., MacKenzie et al. 1998; Lotters et al. 2005; Mannion et al. 2009: Errico et al. 2013). These rates are equal to 0.0317 and 0.0339 for the MSD healthstates, respectively. The developed generator matrix enables us to determine the steady-state probability of each health-state varied according to WIH risk factor changes. 


\subsection{Health-related Performance Variation}

An employee performance level varies due to different work-related health conditions in terms of changing his/her productivity level and working quality.

In terms of the productivity level, previous studies have demonstrated a lower productivity level for an employee who has work-related MSD pain but stays at work than that of a healthy worker. This health-related performance loss is defined as a presenteeism effect (e.g., Lerner et al. 2009: Martimo et al. 2009). Moreover, a new worker usually has less competence (skill level) than an experienced worker leading to a level of productivity loss at work (e.g., Jaber and Khan 2010). In this research, it is assumed that the healthy workers are skilled and $100 \%$ productive. However, the "in-pain" and the new replacement operators have some levels of productivity loss due to work-related MSD and lack of working experience. Numerical details will be defined in the numerical analysis.

In terms of the working quality, any workers can make errors due to human-system interaction when they are at work. These errors cause defective items to be produced. For this study, the sources of the errors are clustered as: A) human errors due to the common features of production systems, contributing inherent variability for the assembly system and generating unpreventable errors. These baseline errors are usually treated as a constant rate in production system analysis (e.g., Myszewski 2010). B) human errors due to the negative effects of the poor HF design of the workplace on working condition (load) contributing to occupational MSD problem (e.g., Erdinc and Vayvay 2008; Erdinc and Yeow 2011).

The human errors in each health-state is, therefore, dominated by a type source of errors. For this study, the constant error rates are assumed for the healthy operator and the replacement operator due to the human baseline errors dominated by the type "A" source of errors. Being in the pain state shows the negative effects of WIH risk factor exposures on the health condition of an operator who is continuing his/her work. In other words, this work-related pain is described as the biological response to WIH risk factor effects, causing an accumulated excessive fatigue over the body of in-pain at work operator (National Research Council 2001; Feuerstein et al. 2004; Bevilacqua et al. 2012). This excessive fatigue can take many forms such as muscular fatigue or general body fatigue (Ahsberg 1998). It usually limits the operator's capability to perform his/her tasks at work correctly by decreasing his/her muscle force capacity and load 
tolerance (e.g., Iridiastadi and Nussbaum 2006; El-Maraghy et al. 2008; Barker and Nussbaum 2011; Dode 2011; Ma et al. 2009; Ham et al. 2012; Zhang et al. 2012). Previous studies discussed the increasing errors of human due to the work-related fatigue (e.g., Chaffin et al. 2006; Ji

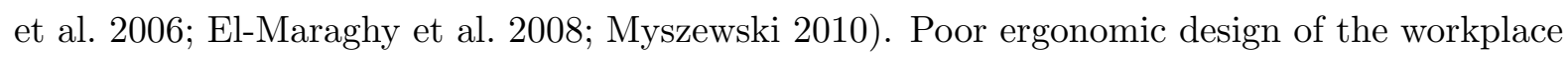
increases the negative effect of WIH risk factor exposure levels causing working conditions (load) to become more difficult for the operator as he/she is generally exposed to higher physical and psychosocial loads, which are contributed to the injury in the first place. Hence, a higher excessive fatigue level is accumulated which reduces the capability of the operator in the pain state to perform working tasks correctly and increases his/her error rate. This rate of errors varies by changing WIH risk factor exposures.

Apart from different human error modeling approach, this study applies the failure function method, the Weibull distribution, to justify the behavior of errors of an assembly line's operator. The applicability of this method in simulating human error rate has been discussed in previous studies (e.g., Giuntini 2000; Myszewski 2010). Weibull distribution supports human error estimation analysis in this study by enabling the users to determine the probability of making errors at each health-state during the given time periods. This probability lets the developed model approximate the number of defective items produced at each workstation. Equation 7.11 demonstrates the probability of making errors, taking into account Weibull density function.

$$
f(t)=\frac{\zeta_{j}^{\bar{h}}}{\rho_{j}^{\bar{h}}}\left(\frac{t-\kappa}{\rho_{j}^{\bar{h}}}\right)^{\zeta_{j}^{\bar{h}}-1} \exp \left[-\left(\frac{t-\kappa}{\rho_{j}^{\bar{h}}}\right)^{\zeta_{j}^{\bar{h}}}\right] \quad \text { and, } \quad p_{j t}^{\bar{h}}=\int_{0}^{t} f(t) d t=1-\exp \left[-\left(\frac{t-\kappa}{\rho_{j}^{\bar{h}}}\right)^{\zeta_{j}^{\bar{h}}}\right] .
$$

To approximate the constant error rate of the healthy and replacement operators in this study, the shape parameter of the Weibull function must be set to 1 . To determine the increasing errors of the operator in the pain state, this slope has a value greater than 1 which is assumed to be changed due to different accumulated excessive fatigue levels. An exponential function is developed to estimate the human fatigue level (e.g., Konz 1998; Dode 2011; Jaber et al. 2013). This function makes a connection between WIH risk factor exposure levels (negative effect of poor HF design) and human errors in the pain state. As shown by Equation (7.12), the developed fatigue function estimates the percentage of maximum excessive fatigue accumulated 
based on working condition (load), which corresponds to the WIH risk factors.

$$
F_{j t}^{w}=1-\exp \left[-\Theta_{j}^{w} t\right]
$$

To achieve reasonable results from the output of the Weibull and fatigue formulas, their parameters are set in a way that their values are within acceptable ranges, as addressed in previous studies (e.g., Bubb 2005).

\subsection{Optimization Model}

The optimization model includes the total cost function of the assembly system, which is generally constrained according to the production flow of the assembly line, the associated operators' performance variations and the expected capacity of each workstation.

$$
\begin{aligned}
& \text { Min } V T C=\sum_{j=1}^{4} \sum_{\bar{h}} c_{j \tau}^{r} \pi_{j \tau}^{\bar{h}} P_{j \tau}^{\bar{h} r}+\sum_{j=1}^{4} \sum_{\bar{h}} c_{j \tau}^{o} \pi_{j \tau}^{\bar{h}} P_{j \tau}^{\bar{h} o}+\sum_{j=1}^{4} \sum_{\bar{h}} c s_{j \tau} \pi_{j \tau}^{\bar{h}} p_{j \tau}^{\bar{h} s} P_{j \tau}^{\bar{h} r} \\
& +\sum_{j=1}^{4} \sum_{\bar{h}} c s_{j \tau} \pi_{j \tau}^{\bar{h}} p_{j \tau}^{\bar{h} s} P_{j \tau}^{\bar{h} o}+\sum_{j=1}^{4} \sum_{\bar{h}} c r_{j \tau} \pi_{j \tau}^{\bar{h}} p_{j \tau}^{\bar{h} r w} P_{j \tau}^{\bar{h} r}+\sum_{j=1}^{4} \sum_{\bar{h}} c r_{j \tau} \pi_{j \tau}^{\bar{h}} p_{j \tau}^{\bar{h} r w} P_{j \tau}^{\bar{h} o} \\
& +I N_{\tau}+I C_{\tau}+s_{\tau} S_{\tau}+\sum_{j=1}^{4} b_{j \tau} B_{j \tau}+\sum_{j=1}^{4} \sum_{\bar{h}} w l_{j \tau}\left(\frac{\pi_{j \tau}^{\bar{h}} P_{j \tau}^{\bar{h} r}+\pi_{j \tau}^{\bar{h}} P_{j \tau}^{\bar{h} o}}{z_{j \tau}^{\bar{h}}}\right) \\
& -\sum_{j=1}^{4} \sum_{\bar{h}} w l_{j \tau}\left(\frac{\pi_{j \tau}^{\bar{h}} P_{j \tau}^{\bar{h} r}+\pi_{j \tau}^{\bar{h}} P_{j \tau}^{\bar{h} o}}{z_{j \tau}^{h}}\right), \quad \text { where, } \quad \bar{h}=\{h, a, w\} .
\end{aligned}
$$

subject to:

$$
\begin{aligned}
& \sum_{\bar{h}} \pi_{4 \tau}^{\bar{h}} P_{4 \tau}^{\bar{h} r}+\sum_{\bar{h}} \pi_{4 \tau}^{\bar{h}} P_{4 \tau}^{\bar{h} o}-\sum_{\bar{h}} \pi_{4 \tau}^{\bar{h}} p_{4 \tau}^{\bar{h} r w} P_{4 \tau}^{\bar{h} r}-\sum_{\bar{h}} \pi_{4 \tau}^{\bar{h}} p_{4 \tau}^{\bar{h} r w} P_{4 \tau}^{\bar{h} o} \\
& -\sum_{\bar{h}} \pi_{4 \tau}^{\bar{h}} p_{4 \tau}^{\bar{h} s} P_{4 \tau}^{\bar{h} r}-\sum_{\bar{h}} \pi_{4 \tau}^{\bar{h}} p_{4 \tau}^{\bar{h} s} P_{4 \tau}^{\bar{h} o}+B_{4 \tau}+S_{\tau}-B_{4 \tau+1} \geq d_{\tau}
\end{aligned}
$$




$$
\begin{gathered}
\sum_{\bar{h}} \pi_{j \tau}^{\bar{h}} P_{j \tau}^{\bar{h} r}+\sum_{\bar{h}} \pi_{j \tau}^{\bar{h}} P_{j \tau}^{\bar{h} o}-\sum_{\bar{h}} \pi_{j \tau}^{\bar{h}} p_{j \tau}^{\bar{h} r w} P_{j \tau}^{\bar{h} r}-\sum_{\bar{h}} \pi_{j \tau}^{\bar{h}} p_{j \tau}^{\bar{h} r w} P_{j \tau}^{\bar{h} o}-\sum_{\bar{h}} \pi_{j \tau}^{\bar{h}} p_{j \tau}^{\bar{h} s} P_{j \tau}^{\bar{h} r} \\
-\sum_{\bar{h}} \pi_{j \tau}^{\bar{h}} p_{j \tau}^{\bar{h} s} P_{j \tau}^{\bar{h} o}+\sum_{\bar{h}} \pi_{j+1 \tau}^{\bar{h}} p_{j+1 \tau}^{\bar{h} r w} P_{j+1 \tau}^{\bar{h} r}+\sum_{\bar{h}} \pi_{j+1 \tau}^{\bar{h}} p_{j+1 \tau}^{\bar{h} r w} P_{j+1 \tau}^{\bar{h} o} \\
-\sum_{\bar{h}} \pi_{j+1 \tau}^{\bar{h}} P_{j+1 \tau}^{\bar{h} r}-\sum_{\bar{h}} \pi_{j+1 \tau}^{\bar{h}} P_{j+1 \tau}^{\bar{h} o}+B_{j \tau}-B_{j \tau+1} \geq 0 . \quad \text { where, } j=\{1,2,3\} \\
P_{j \tau}^{\bar{h} r} \leq s h_{j \tau}^{r} m_{j \tau} z_{j \tau}^{\bar{h}} \quad \text { and } \quad P_{j \tau}^{\bar{h} o} \leq s h_{j \tau}^{o} m_{j \tau} z_{j \tau}^{\bar{h}} . \\
P_{j \tau}^{\bar{h} r}, P_{j \tau}^{\bar{h} o}, S_{\tau} \text { and } B_{j \tau} \geq 0 . \quad \text { and integers. }
\end{gathered}
$$

$$
\boldsymbol{B}_{\tau} \in \boldsymbol{I}
$$

$$
B_{j \tau} \in \boldsymbol{B}_{\tau}
$$

The objective function, Equation (7.13), includes the expected production cost of the assembly system during regular-time and over-time shifts, inventory, subcontracting and qualityrelated expenses and Occupational Health and Safety (OHS) costs. For this study, when an error occurs, the probability of producing scrap products, $p_{j \tau}^{\bar{h}}$, is assumed to be the same as the rework products, $p_{j \tau}^{\bar{h} r w}$. Moreover, for each operator, the per unit scrapping cost is assumed to be 2 times of the per unit reworking cost.

Constraint (7.14 and 7.15 control the flow of product from station 1 to station 4 . The values of the demand at time $\tau$ are set according to the lattice model. Constraint (7.16) indicates the production capacity of each workstation in time $\tau$ which is dominated by the health-related productivity levels of the associated operator. 22 working days is set for time-step $\tau$. Constraint 7.18 shows the possible inventory levels during time $\tau$. Since the model considers 3 buffer levels in this study, $\boldsymbol{I}$ includes 3 values. Constraint (7.19) indicates the inventory level of each station during during time $\tau$.

Insurance premium is one of the OHS indicators representing the effects of medical treat- 
ments and compensation expenses spent by the injured operators in the developed model. This study applies an OHS performance sensitive approach (e.g., Riel and Imbeau 1998) to determine the total insurance premium cost of the operators. Equation 7.20 shows this cost item adjusted in a way to include WIH effects. AIN, is the unit cost of the insurance premium as per dollar of medical treatment and compensation expenses credited per dollar of insured salaries. inwj is defined as the average of the annual insurable salary per assembly lines' operator. In order to estimate the average of monthly insurance cost, the insurance function's parameters are calibrated according to Workplace Safety and Insurance Board (WSIB) of Ontario, Canada and Ontario Ministry of Labor annul reports (e.g., HRSDC 2010; WSIB 2011)

$$
I N_{\tau}=4 A I N \sum_{j=1}^{4} i n w_{j}\left(\sum_{j=1}^{4} \sum_{\bar{h}=w, a} m e_{\tau} \pi_{j \tau}^{\bar{h}} m_{j \tau}\right) .
$$

The indirect OHS costs include financial items spent by the company to cover all expenses of selecting, administration and training of the new replacement workers. These types of expenses are not usually specified as a separate financial items in the company's financial reporting. But, several studies were attempted to estimate these expenses in comparison with medical care and benefit costs (e.g., Leigh 2011). Taking into account WSIB conservative estimation (WSIB 2010a), the developed optimization model includes OHS indirect costs, which are estimated as 3 times of medical care and rehabilitation expenses. Equation (7.21) shows the indirect OHS costs estimation's formula.

$$
I C_{\tau}=3 \sum_{j=1}^{4} \sum_{\bar{h}=w, a} m e_{\tau} \pi_{j \tau}^{\bar{h}} m_{j \tau}
$$

According to the last cost items in the objective function, wage loss is another OHS performance-related cost factor aiming to determine the amount of payments for working hours which are lost due to the operators' health-related productivity loss during time-step $\tau$.

\subsection{Dynamic Programming}

There are two steps in order to determine the present value of the optimum total cost of the assembly system: first, the total cost is minimized at each node of the demand developed by the 
lattice model. This procedure begins from the end of the product life cycle $(\tau=60)$. Second, by folding back to the previous time-step, the backward dynamic programing enables us to estimate the present value of the intermediary nodes. Other than the demand value at time step $\tau$, the minimized cost at each node is also conditioned on the inventory levels of the buffers, $B_{\tau}$, at time $\tau$ and $\tau+1$. The present value of an intermediary node includes the minimized total cost at the underlying demand value plus the expected cost value discounted from the next time period and holding cost of changing inventory levels. Equation 7.22 indicates the present value of the optimum total cost of the assembly line for the intermediary node of the developed pentanomial lattice.

$$
\begin{aligned}
\operatorname{PVTC}\left(q, \boldsymbol{B}_{\boldsymbol{\tau}}, \hat{\psi}, \tau\right)= & \min _{\boldsymbol{I}}\left[V T C\left(q, \boldsymbol{B}_{\boldsymbol{\tau}}, \tau\right)+\operatorname{EVTC}\left(q, \boldsymbol{B}_{\boldsymbol{\tau}+\mathbf{1}}, \hat{\psi}, \tau\right)+H\left(\boldsymbol{B}_{\boldsymbol{\tau}}, \boldsymbol{B}_{\boldsymbol{\tau}+\mathbf{1}}\right)\right] \\
& \text { where } \quad B_{j \tau} \in \boldsymbol{B}_{\boldsymbol{\tau}} \quad \text { and } \quad \boldsymbol{B}_{\boldsymbol{\tau}} \in \boldsymbol{I} .
\end{aligned}
$$

$\operatorname{VTC}\left(q, \boldsymbol{B}_{\boldsymbol{\tau}}, \tau\right)$ is the minimized total cost of the assembly system at time $\tau$ which is conditioned on the demand value at its level, $q$, and the current buffer inventory level work stations. $\operatorname{EVTC}\left(q, \boldsymbol{B}_{\boldsymbol{\tau}+\mathbf{1}}, \hat{\psi}, \tau\right)$ is the discounted expected value of the optimum total cost. This discounted cost is dependent on the current demand regime, $\hat{\psi}$, since each regime is constructed by different branches. The expected value for the growth regime is shown by Equation (7.23)

$$
\begin{gathered}
\operatorname{EVTC}\left(q, \boldsymbol{B}_{\boldsymbol{\tau}+\mathbf{1}}, g, \tau\right)=\exp ^{-i / 12}\left(( 1 - k _ { \tau } ) \left\{\delta_{(g, u)} \operatorname{PVTC}\left(q, \boldsymbol{B}_{\boldsymbol{\tau}+\mathbf{1}}, g, \tau+1\right)+\right.\right. \\
\left.\delta_{(g, m)} \operatorname{PVTC}\left(q+2, \boldsymbol{B}_{\boldsymbol{\tau}+\mathbf{1}}, g, \tau+1\right)+\delta_{(g, d)} \operatorname{PVTC}\left(q+4, \boldsymbol{B}_{\boldsymbol{\tau}+\mathbf{1}}, g, \tau+1\right)\right\}+ \\
\left(k_{\tau}\right)\left\{\delta_{(d, u)} \operatorname{PVTC}\left(q+1, \boldsymbol{B}_{\boldsymbol{\tau}+\mathbf{1}}, d, \tau+1\right)+\delta_{(d, m)} \operatorname{PVTC}\left(q+2, \boldsymbol{B}_{\boldsymbol{\tau}+\mathbf{1}}, d, \tau+1\right)+\right. \\
\left.\left.\delta_{(d, d)} \operatorname{PVTC}\left(q+3, \boldsymbol{B}_{\boldsymbol{\tau}+\mathbf{1}}, d, \tau+1\right)\right\}\right) .
\end{gathered}
$$

" $g$ " and " $d$ " are representing the growth and decay regimes. $k_{\tau}$ is the probability of switching from the growth to the decay regime which is cumulatively distributed through the product life cycle. The calculation of the discounted expected total cost of the decay regime is indicated by Equation (7.24). Since the current regime of the demand is decay, the demand remains in that regime. 


$$
\begin{aligned}
& \operatorname{EVTC}\left(q, \boldsymbol{B}_{\boldsymbol{\tau}+\mathbf{1}}, d, \tau\right)=\exp ^{-i / 12}\left(\delta_{(d, u)} \operatorname{PVTC}\left(q+1, \boldsymbol{B}_{\tau+\mathbf{1}}, d, \tau+1\right)+\right. \\
& \left.\delta_{(d, m)} \operatorname{PVTC}\left(q+2, \boldsymbol{B}_{\boldsymbol{\tau}+\mathbf{1}}, d, \tau+1\right)+\delta_{(d, d)} \operatorname{PVTC}\left(q+3, \boldsymbol{B}_{\boldsymbol{\tau}+\mathbf{1}}, d, \tau+1\right)\right) .
\end{aligned}
$$

As shown by Equation 7.22 , the present value of the optimum total cost at time $\tau$ is conditioned on the buffer levels of workstations at $\tau+1$. With a given inventory level to the buffers of the workstations at time $\tau$, there are three possibilities for buffer inventory levels at time $\tau+1$. Hence, the $H\left(\boldsymbol{B}_{\boldsymbol{\tau}}, \boldsymbol{B}_{\boldsymbol{\tau}+\mathbf{1}}\right)$ shows the holding cost if the inventory level of the buffers at time $\tau+1$ are different from the given values at time $\tau$. Equation 7.25 demonstrates the calculation of the holding cost.

$$
H\left(\boldsymbol{B}_{\boldsymbol{\tau}}, \boldsymbol{B}_{\boldsymbol{\tau}+\mathbf{1}}\right)=\sum_{j=1}^{4} h c_{j}\left(B_{j \tau+1}-B_{j \tau}\right) .
$$

Finally, this folding procedure continues recursively to determine the expected optimum total cost of the assembly line at the beginning of the product life cycle.

\subsection{Numerical Analysis}

This section numerically tests the developed model to evaluate the effects of WIH risk factors and the dynamic behavior of the customer demand. It is organized according to two scenarios. The first scenario focuses on the valuation of the optimum total cost of the assembly system in the presence of $\mathrm{HF}$ variations while there is a given set of parameters for the pentanomial lattice to replicate uncertain demand throughout the product life cycle. Table 7.1 indicates the parameters and assumptions which have been set in a way that the value option model becomes feasible. To consider WIH effects, this study applies low back pain risk factor information, collected from an automotive company in Ontario, Canada (Kerr 1997). In the second scenario, the robustness of the value option model is examined in the presence of different setups for the stochastic demand. The efforts are focused on analyzing the effects of demand's regime parameters while a given set of HF are integrated into the assembly line's cost optimization model. 


\begin{tabular}{|c|c|}
\hline Parameters & Description \\
\hline$c_{1 \tau}^{r}=\$ 20$ & It is linearly increased by $\$ 5$ per product for each consecutive workstation \\
$c_{1 \tau}^{o}=\$ 25$ & It is linearly increased by $\$ 5$ per product for each consecutive workstation \\
$c s_{1 \tau}=\$ 100$ & It is linearly increased by $\$ 5$ per product for each consecutive workstation \\
$c r_{1 \tau}=\$ 50$ & It is linearly increased by $\$ 5$ per product for each consecutive workstation \\
$m e_{\tau}=\$ 74$ & Determined according to the Ontario Ministry of Labor report $($ HRSDC 2010$)$ \\
$d_{1}=1,600$ units & It is the value for the common node of the regimes \\
& and initialized at the beginning of the cycle \\
$b_{1 \tau}=\$ 5$ & It is linearly increased by $\$ 2$ per product for each consecutive buffer \\
$\rho_{j}^{w}=185$ & When all WIH risk factor are set to their mean values \\
$\zeta_{j}^{w}=1.5$ & When all WIH risk factor are set to their mean values \\
$F_{j t}^{w}=25 \%$ & When all WIH risk factor are set to their mean values \\
$\kappa=0$ & for all health-states \\
$\rho_{j}^{\bar{h}}=16,000$ & $\bar{h}=h, a$ \\
$\zeta_{j}^{\bar{h}}=1$ & $\bar{h}=h, a$ \\
$\boldsymbol{I}=10,25,40$ units & The possible buffer levels of the workstations \\
$i=0.10$ & Annual time value money risk \\
$\sigma_{g}=0.25$ & The volatility value of the growth regime \\
$\sigma_{d}=0.10$ & The volatility value of the decay regime \\
$\xi_{g}=0.25$ & The mean value of the growth regime \\
$\xi_{d}=-0.20$ & The mean value of the decay regime \\
$h c_{j}=\$ 32$ & Unit cost of changing buffer capacity for all workstations \\
$i n w_{j}=\$ 40,000$ & for each operator \\
$w l_{j \tau}=\$ 20$ & for all health-states \\
$s h_{j \tau}^{r}=8$ hours & for all heath-states \\
$s h_{j \tau}^{o}=4$ hours & for all health-states \\
$m_{j \tau}=22$ days & for all health-states \\
$k_{\tau}$ & mean $=30$ and standard deviations $=8$ \\
$s_{\tau}=\$ 175$ & Ts a ped per outsourced product \\
\hline
\end{tabular}

Table 7.1: The value of the system's parameters and the corresponding assumptions made for the example numerical analysis

For the numerical model testing, the healthy operator is assumed to be $100 \%$ productive. However, the in-pain and replacement operators both have productivity losses. The HF literature suggests that the health-related productivity loss vary between $3 \%$ and $34 \%$ (e.g., Allen et al. 2005; Meerding et al. 2005; Oxenburgh and Marlow 2005, Lerner et al. 2009; Martimo et al. 2009, Jaber and Khan 2010). For this study, the productivity loss for the "in-pain" at work and replaced operators are cautiously estimated as $16.6 \%$ and $10 \%$, respectively, determined based on the average of the reported productivity losses from previous studies. 


\subsubsection{Scenario 1: Low Back Pain Risk Factor Effects}

The primary low back pain WIH information is extracted from Kerr (1997), accomplished a comprehensive epidemiology study in an automotive company. The risk factor definitions and associated parameters are indicating as: a) cumulative spinal compression loading. This physical risk factor is determined based on the integrated instantaneous loads on L4/L5 spine over a shift. It is usually measured according to Mega Newton seconds per full-time shift (MNs/shift); b) peak hand loading is scaled based on the maximum force exerted in the shift, measured in the Kilogram scale $(\mathrm{kg})$; c) peak shear force. It is defined based on the maximum average of shear forces acting on L4/L5 lumbar spine, measured in newtons (N); d) job control is a psychosocial risk factor which is distinguishing the perception of an operator regarding job requirements, task repetitions, and the operator's role in making decisions; e) perceived physical exertion measures the perception of operators regarding physical demands of their jobs; f) social environment is considered as an operator's perception of the social atmosphere of the workplace; and g) over-educated level is about the perception of operators education level compared to that of their co-workers performing similar jobs. The mean values of the given risk factors observed by the Kerr study are shown by Table 7.2 (Kerr 1997). More details were described in chapter 5 (page 50) with regard to scaling psychosocial risk factor exposure levels.

\begin{tabular}{|c|c|c|c|c|}
\hline Risk factors & $\begin{array}{l}\text { Measures } \\
(s t d)\end{array}$ & $\begin{array}{c}\text { Odds Ratio(ODR) } \\
95 \% \text { CI }\end{array}$ & $\begin{array}{c}\text { Coefficient correlation } \\
\beta_{n j}=(\ln \text { ODR }) / a_{n j}\end{array}$ & $\begin{array}{c}\text { Constant coefficient } \\
\alpha_{0 j}\end{array}$ \\
\hline Peak hand load $(K g)$ & $14.3(12.4)$ & $1.8(1.15-2.87)$ & 0.035 & \multirow{8}{*}{-1.339} \\
\hline Peak shear $N$ & $354(159)$ & $1.9(1.16-3.17)$ & 0.003 & \\
\hline Cumulative compression & 12.2 & $1.9(1.26-2.93)$ & $8.2 \mathrm{E}-08$ & \\
\hline MNs/shift & $(0.665)$ & & & \\
\hline Job control & $56.5(19.2)$ & $1.9(0.89-4.09)$ & -0.020 & \\
\hline Perceived exertion & $6(1.6)$ & $3.2(1.91-5.70)$ & 0.582 & \\
\hline Social environment & $12.4(1.7)$ & $2.8(1.42-5.84)$ & -0.343 & \\
\hline Over educated level & $($ yes $/$ no $)=1 / 0$ & $2.3(1.09-5.15)$ & 0.833 & \\
\hline
\end{tabular}

Table 7.2: Low back pain WIH risk factor measures and parameters used in the Logistic regression

In the Kerr et al. (2001) epidemiological analysis, the odds ratios indicate the relative contributions of the WIH risk factors by comparing subjects who have low back pain, called "cases", with workers who did not report the pain/injury issue but are otherwise similar, called "controls". They help users figure out how much more likely it is that an operator, who is 
exposed to a given risk factor will gain work-related pain through comparing the "cases" with the "controls" (e.g., Kerr et al. 2001). Parameter $a_{n j}$ shows the average difference of a given risk factor measured between the "cases" and the "controls".

In the case-control statistical analysis like Kerr et al. (2001), the "case" group members are usually designated based on a non-randomized sampling method. Hence, the Logistic regression needs a calibration to calculate the correct proportion value (King and Zeng 2002). Equation (7.26) adjusts the constant coefficient of the Logistic regression formula, $\hat{\alpha, ~ a c c o r d i n g ~}$ to the combination of the population fraction of employees reporting low back pain, $\chi$, and the prevalence rate of employees reporting low back pain in the Kerr (1997) study, $y^{\prime}$.

$$
\alpha_{0 j}=\hat{\alpha}-\ln \left(\frac{1-\chi}{\chi} \times \frac{y^{\prime}}{1-y^{\prime}}\right)=-1.2-\ln (1.5 \times 0.766)=-1.339 \text {. }
$$

By applying Equations (7.8), 7.9), and 7.10), the health-related generator matrix is completed. Hence, the steady-state probability of each health-state is determined according to the given low back pain risk factor exposure levels.

The health-related probability of making errors is determined according to Equation 7.11 - It is assumed to be as $0.137 \%$ for the healthy and replacement operators. For the in-pain state operators, the probability is assumed to be as $4 \%$ when all low back pain risk factors are set to their mean exposure levels, described by Table 7.2. These probability values are within the acceptable ranges: for the base line human errors (e.g., Bubb 2005), and for human making errors due to work-related health problems determined by previous literature (e.g., Yeow and Sen 2006; Ruckart and Burgess 2007). We use the fatigue formula, Equation 7.12, to determine the "in-pain" operator error rate which is varying due to low back pain risk factor changes (working condition). $F_{j t}^{w}=0.25$ is assigned when all low back pain risk factors are set to their mean exposure levels, described by Table 7.2. This assumption is consistent with the fatigue level affecting human performance (e.g., Parhizi and Pasupathy 2013). This fatigue level is called "medium fatigue" in this chapter. By substituting the "medium fatigue" level in Equation 7.12, the associated workload level, $\Theta_{j}^{w}$, is equal to 0.013. This workload measure is called, here, "medium workload" since it is estimated when all WIH risk factors are set to their mean levels. WIH risk factor exposure changes vary the probability of the in-pain state and workload level. For the new $\Theta_{j}^{w}$ level, the value of "medium workload" is proportionally 
changed according to the ratio of the new probability of the "in-pain" state to the probability of being in "pain" state when all risk factors are set to their mean exposure levels. After wards, the new fatigue level is determined by substituting the new workload measure in Equation (7.12). The slope parameter, $\zeta_{j}^{w}$, of the Weibull distribution is also proportionally changed taking into account the ratio of the new fatigue level to the "medium fatigue" level in order to estimate the new probability of making errors by the "in-pain" operators.

To examine low back pain WIH risk factor effects in this scenario, first, all risk factors are set to their mean exposure levels. Then, individually, each risk factor is changed within a distance of \pm 3 standard deviations (std) over its mean measure for all operators. The steadystate probability of each health-state and the probability of making errors are determined accordingly. Then, with regard to each set of WIH risk factor exposure levels, Equation (7.22) is used to the valuation of the total cost of the assembly system within the product life cycle. Table (7.3) and (7.4) indicate the effect of the examples of physical and psychosocial risk factor changes on the health-condition, expected error rate of an operator and cost per unit of the assembly line's final product, determined according to the option value approach. These tables include the estimated data when demand starts with the growth regime at the beginning of the product life cycle. In general, when the negative effect of WIH risk factor increases, the employees have a higher chance to be injured and being absent or being in-pain at work with the associated productivity and quality losses. Therefore, the expected rate of producing defective items and the expected cost at the beginning of the product life cycle increase, accordingly. By comparing the buffer level change effects, it is concluded that the cost per unit of the final product decreases as the inventory level increases. However, these cost changes are negligible in comparison with the effects of physical and psychosocial risk factors on the cost per unit of the product.

As an example for the physical risk factor, working conditions may vary the cumulative load of the operators between 5.5 to $38.8 \mathrm{MNs}$ /shift. All the other low back pain risk factors are assumed to be set to their mean levels. At the lowest cumulative loading exposure level, an operator in the assembly system has $14.4 \%$ chance to be injured at work.

Moreover, he or she may go to the absent and be replaced by the new worker with the probability of $7.74 \%$. Hence, his/her expected rate of producing defective becomes minimum, which is equal to $0.32 \%$. With the capacity of 10 units for the buffers, the present cost of 


\begin{tabular}{|c|c|c|c|c|c|c|c|}
\hline Risk Factors & Exposure level & $\pi_{j \tau}^{w}$ & $F_{j t}^{w}$ & Expected human & \multicolumn{3}{|c|}{ Cost per unit $(\$)$} \\
\cline { 6 - 8 } & & & $(\%)$ & error rate $(\%)$ & $B_{j 1}=10$ & $B_{j 1}=25$ & $B_{j 1}=40$ \\
\hline & 5.5 & 0.144 & 18.7 & 0.32 & 79.61 & 79.59 & 79.56 \\
Cumulative load & 12.2 & 0.201 & 25 & 0.92 & 81.88 & 81.86 & 81.85 \\
(MNs/shift) & 18.8 & 0.26 & 31.1 & 2.02 & 83.53 & 83.52 & 83.50 \\
& 25.5 & 0.315 & 36.2 & 3.38 & 86.05 & 86.03 & 86.00 \\
& 32.1 & 0.359 & 40.1 & 4.67 & 90.13 & 90.11 & 90.08 \\
& 38.1 & 0.391 & 42.8 & 5.69 & 95.31 & 95.28 & 95.23 \\
\hline & 1.9 & 0.156 & 20 & 0.40 & 79.89 & 79.88 & 81.85 \\
Peak load & 14.3 & 0.201 & 25 & 0.92 & 81.88 & 81.87 & 77.78 \\
(kg) & 26.7 & 0.248 & 29.8 & 1.75 & 83.18 & 83.17 & 83.15 \\
& 39.1 & 0.292 & 34.1 & 2.79 & 84.76 & 84.74 & 84.73 \\
& 51.5 & 0.331 & 37.7 & 3.86 & 85.95 & 85.93 & 85.90 \\
Without HF & 63.9 & 0.364 & 40.5 & 4.82 & 90.74 & 90.71 & 90.68 \\
\hline
\end{tabular}

Table 7.3: The examples of work-related health, operational and financial effects of physical WIH risk factors with the growth regime at the beginning of the life cycle (The table includes: physical risk factors' exposure level, the probability of being in-pain, the average excessive fatigue level, the expected error rate of the operators and the cost per unit of the final product for each level of inventory)

scraping products is equal to $\$ 35,076$. Therefore, the cost per unit of product is estimated as $\$ 79.61$ at the beginning of the product life cycle. Due to increasing the cumulative load, working condition becomes worse which is increasing the chance of being injured. Therefore, the output of the assembly system is more affected by the productivity loss and quality deficits of the in-pain and replacement workers. With $38.8 \mathrm{MNs} / \mathrm{shift}$, the chance of being in-pain at work or being absent becomes $39.11 \%$ and $19.11 \%$, respectively. Furthermore, the expected error rate of the operators reaches to $5.69 \%$ due to the negative effect of working load on their body and developing more excessive fatigue. The expected cost of scraping products is then equal to $\$ 257,475$. These health-related operational consequences increase the expected cost per unit of final product to $\$ 95.31$. As the cumulative load increases, the efficiency of the assembly system reduces due to the operators' work-related productivity and quality losses. Therefore, more products are outsourced to satisfy the demand. With the capacity of 10 units for the buffers, the subcontracting cost changes between $\$ 345,121$ to $\$ 1,127,710$ when the cumulative compression is varied between 5.5 and $38.8 \mathrm{MNs} / \mathrm{shift}$, respectively. There is a similar behavior for the value options in the presence of the other physical risk factor changes.

Table 7.4 shows the effects of examining the developed model according to job control 


\begin{tabular}{|c|c|c|c|c|c|c|c|}
\hline Risk Factors & Exposure level & $\pi_{j \tau}^{w}$ & $F_{j t}^{w}$ & Expected human & \multicolumn{3}{|c|}{ Cost per unit $(\$)$} \\
\cline { 5 - 7 } & & & $(\%)$ & error rate $(\%)$ & $B_{j 1}=10$ & $B_{j 1}=25$ & $B_{j 1}=40$ \\
\hline \multirow{5}{*}{ Perceived exertion } & 1.2 & 0.021 & 3.10 & 0.130 & 78.54 & 78.52 & 78.51 \\
& 2.8 & 0.052 & 7.11 & 0.135 & 78.66 & 78.65 & 78.63 \\
& 4.4 & 0.11 & 14.60 & 0.17 & 79.04 & 79.03 & 79.01 \\
& 6 & 0.201 & 25.0 & 0.92 & 81.88 & 81.87 & 81.85 \\
& 7.6 & 0.299 & 34.8 & 2.30 & 85.12 & 85.10 & 85.03 \\
& 9.2 & 0.373 & 41.3 & 5.10 & 92.31 & 92.29 & 92.26 \\
& 10.8 & 0.414 & 44.7 & 6.50 & 130.74 & 130.62 & 130.49 \\
\hline \multirow{5}{*}{ Job control } & 18.1 & 0.283 & 33.3 & 2.60 & 84.38 & 84.35 & 84.34 \\
& 37.3 & 0.244 & 29.4 & 1.70 & 83.06 & 83.04 & 83.03 \\
& 56.5 & 0.201 & 25.0 & 0.92 & 81.88 & 81.87 & 81.85 \\
& 75.7 & 0.161 & 20.5 & 0.44 & 80.02 & 80.00 & 79.98 \\
& 94.9 & 0.124 & 16.3 & 0.21 & 79.22 & 79.21 & 79.19 \\
& 114.1 & 0.093 & 12.5 & 0.14 & 78.89 & 78.87 & 78.86 \\
\hline \multirow{5}{*}{ Social environment } & 7.3 & 0.360 & 40.8 & 4.90 & 91.05 & 91.02 & 90.99 \\
& 9 & 0.320 & 36.80 & 3.60 & 83.33 & 83.30 & 83.28 \\
& 10.7 & 0.265 & 32.5 & 2.10 & 83.65 & 83.64 & 83.62 \\
& 12.4 & 0.201 & 25.0 & 0.92 & 81.88 & 81.87 & 81.86 \\
& 14.1 & 0.141 & 18.3 & 0.30 & 79.53 & 79.52 & 79.50 \\
& 15.8 & 0.092 & 12.3 & 0.14 & 78.88 & 78.86 & 78.85 \\
\hline
\end{tabular}

Table 7.4: The examples of work-related health, operational and financial effects of psychosocial WIH risk factors with the growth regime at the beginning of the life cycle (The table includes: physical risk factors' exposure level, the probability of being in-pain, the average excessive fatigue level, the expected error rate of the operators and the cost per unit of the final product for each level of inventory)

variation. At the lowest exposure level, 18.1, the operators psychosocially perceive minimum control over their jobs. Under these conditions, they have the highest chance of having workrelated low back problems. It is equal to $28.8 \%$ and $14.6 \%$ for the in-pain and absent states, respectively. With the capacity of 10 units for the buffers, the expected rate of producing defective products reaches to $2.6 \%$ causing $\$ 109,700$ scarping expenses. Therefore, the cost per unit of the final product is equal to $\$ 84.38$. By improving the psychosocial atmosphere of the working environment, the operators perceive a higher control over their assigned tasks. The chance of being injured reduces causing less health-related performance losses. Hence, with 114.1 job control exposure level, the cost per unit is equal to $\$ 78.89$ (6.5\% reduction). The mechanism of job control in developing MSD was investigated in previous studies (e.g., Devereux et al. 1999, MacFarlane and Silman 2000).

The results shown by the Tables 7.3 and 7.4 have been estimated based on the growing regime for the demand at the beginning of the product life cycle. However, according to the developed options value model, the demand can follow the decay regime throughout the life cycle. To 

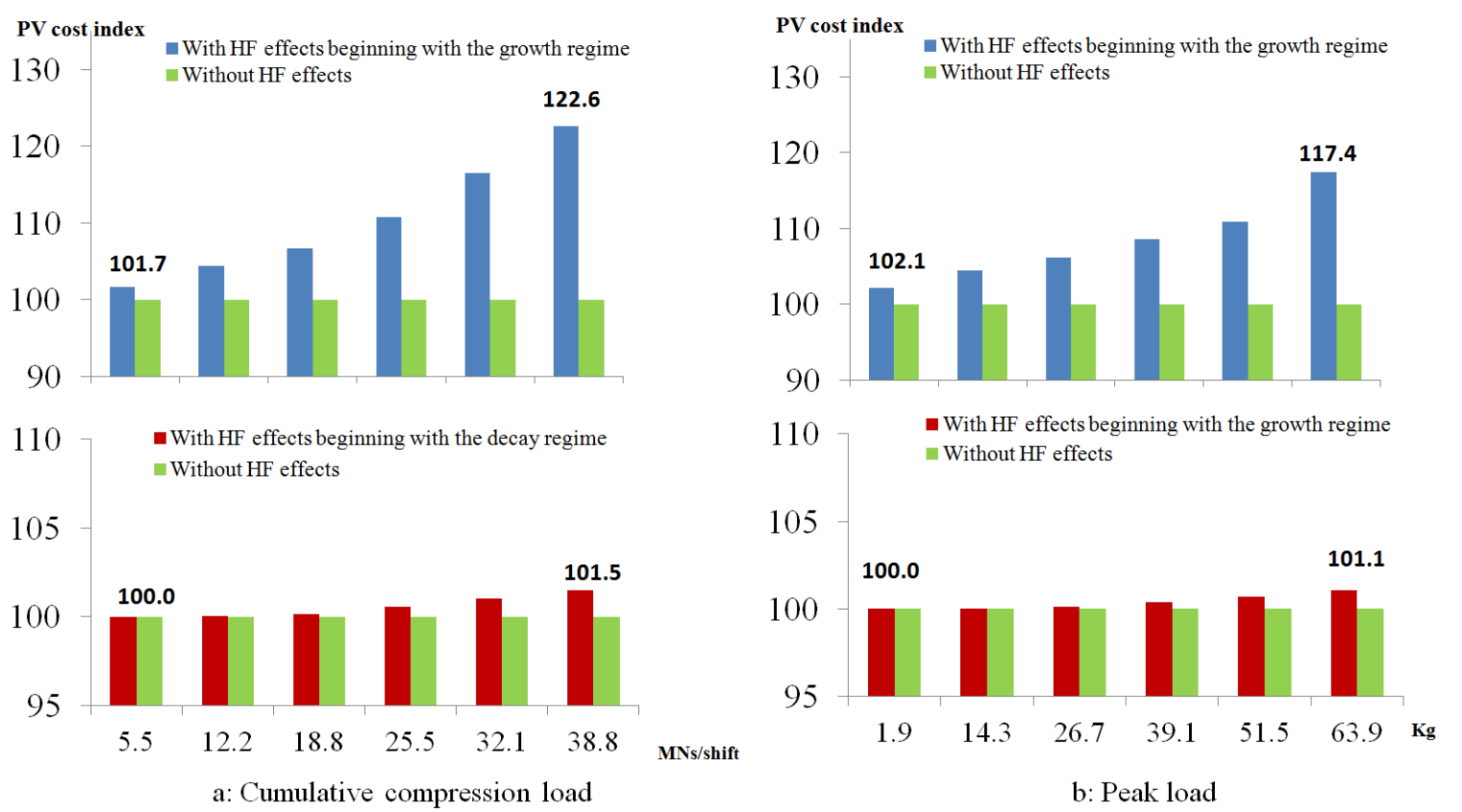

Figure 7.2: The valuation of the total cost (present value cost index) versus physical risk factor changes $\left(B_{j 1}=10\right.$ units, the above figures show the model's results with the growth regime at the beginning of the life cycle, the bottom figures show the model's results with the decay regime at the beginning of the life cycle)

better understand the effect of WIH risk factor on the expect total cost of the assembly system, Figures 7.2 and 7.3 indicate the expected cost index variation of the assembly system when the product life cycle either starts with the growth regime or the decay regime. The cost index is determined at each risk factor exposure level and compared to the result of the model without including HF. Without HF effects, the expected total cost is equal to $\$ 8,664,220$ and $\$ 4,724,280$ for the growth and decay regimes, respectively. These expected cost values are used as the baseline cost index of each regime, which are equal to 100. According to the model without HF effects, it is assumed that the operators are $100 \%$ productive with the baseline human errors at work. Their performances are not affected by changing WIH risk levels and the associated optimization model does not include indirect OHS and wage loss costs, due to ignoring WIH effects.

As demonstrated by Figures $(7.2$ ) , the cost index varies between 101.70 and 122.60 when cumulative load level changes at the growth regime. However, with the decay regime at the beginning of the product life cycle, the increment rate of cost index reduces. The same behavior 
is shown by $(7.2 \mathrm{~b})$ versus the peak load changes. Figures $(7.3 \mathrm{a})$ demonstrates the cost index
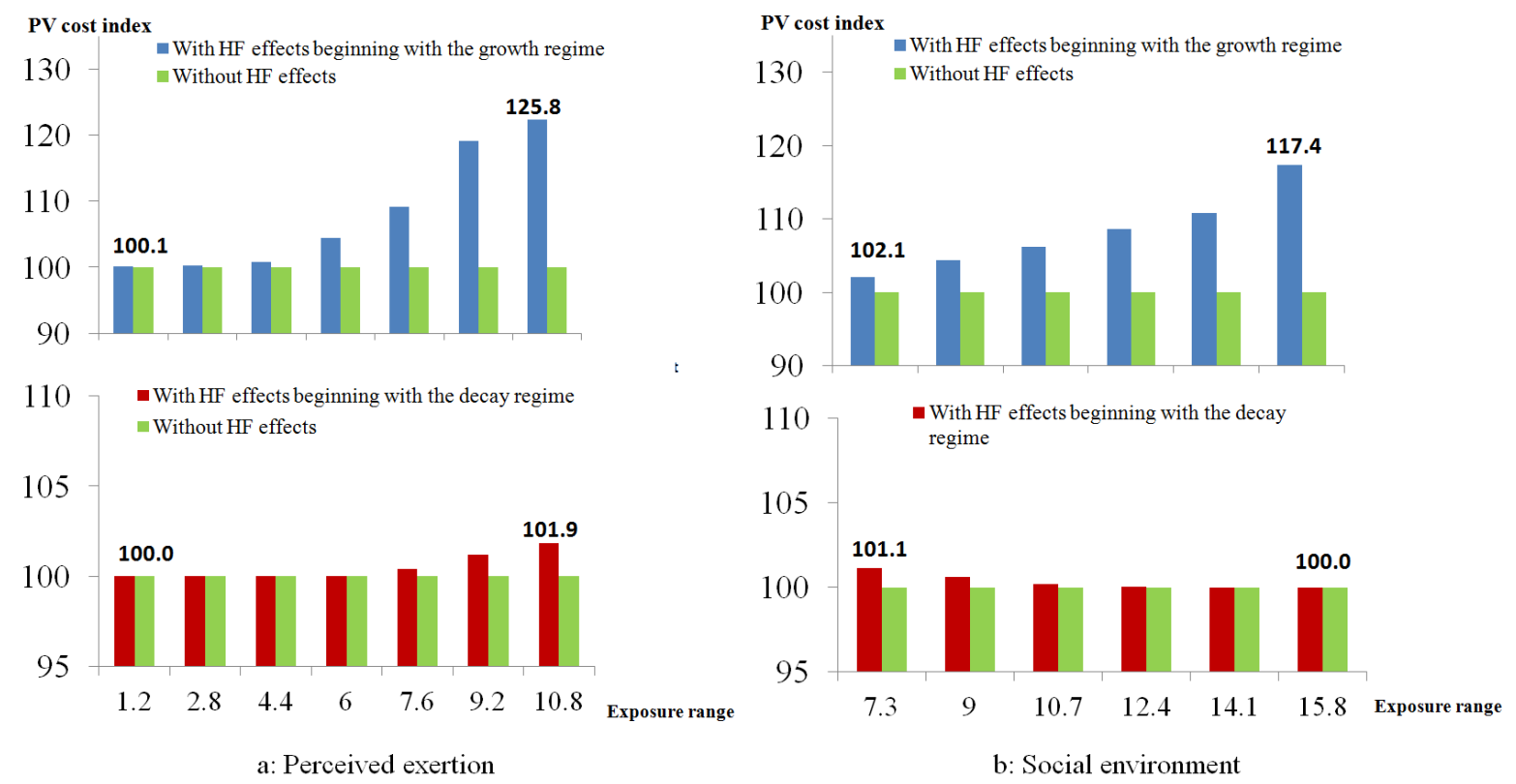

Figure 7.3: The valuation of the total cost (present value cost index) versus psychosocial risk factor changes $\left(B_{j 1}=10\right.$ units, the above figures show the model's results with the growth regime at the beginning of the life cycle, the bottom figures show the model's results with the decay regime at the beginning of the life cycle)

variations versus the operators' perceived exertion. It changes between 100.1 to 125.8 when the demand starts with the growth regime. With the decay regime at the start time of the life cycle, this variation would be between 100.0 and 101.9. The trend is similar when the social environment of the working environment changes.

\subsubsection{Scenario 2: Stochastic Demand Parameter Variations}

The aim of the scenario 2 is to examine the effects of the demand parameter changes on the expected total cost of the assembly system. To do so, it is focused on the variation of $\xi_{\psi}$ and $\sigma_{\psi}$, the mean and volatility of the growth and decay regimes to evaluate the stochastic behavior of the demand, modeled according to the pentanomial lattice. Furthermore, the option cost values determined at the beginning of the product life cycle are compared with and without including HF. To model HF effects, the low back pain risk factors are set to their mean exposure levels, as described by Table 7.2. In order to estimate the expected total cost at the beginning 
of the product life cycle, the associated steady-state probability of being in each work-related health-state and employee health-related performance variations are applied.

\section{Growth Regime Parameter Variation Effects}

As described by section 7.2, the life cycle has the highest chance to follow increasing demand (growth regime) at the beginning of the production. Figures (7.4) and (7.6) demonstrate the impact of the mean and the volatility changes of the growth regime on the expected total cost behavior of the assembly system.

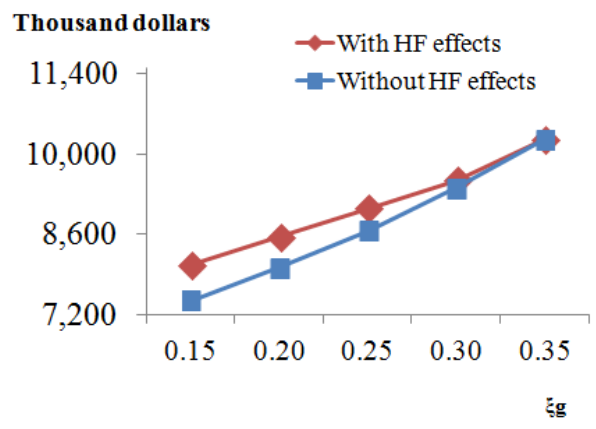

Figure 7.4: The valuation of the total cost versus the mean of the growth regime changes $\left(B_{j 1}\right.$ $=10$ units)

Figure (7.4) demonstrates a growing trend of the total cost versus the mean of the growth regime, $\xi_{g}$, changes. It is concluded that by increasing the mean of the growth regime, the density of high demand values increases causing the life cycle to likely remain in the growth for a longer period of the time. The results of the numerical analysis show that the expected total cost increases when $\xi_{g}$ is changed from 0.15 to 0.35 . With $\xi_{g}=0.15$ and including HF effects, the total cost is equal to $\$ 8,058,155$ for the growth regime, determined by the pentanomial lattice model. By increasing the mean level to 0.35, the expected demand volume becomes higher throughout the product life cycle. Hence, the total cost value is equal to $\$ 10,251,100$, ( $27 \%$ growth). This experimental analysis shows that the increment of $\xi_{g}$ lets the growth regime dominate the estimation of the demand value and the associated branch probabilities at each node of the pentanomial lattice. Thus, with a higher mean value, the expected demand of the assembled product considerably increases. It implies that the life cycle will likely remain in the growth regime. Figure 7.5 compares the demand and branch probabilities of the lattice with 
respect to increasing the mean of the growth regime from 0.15 to 0.35 .

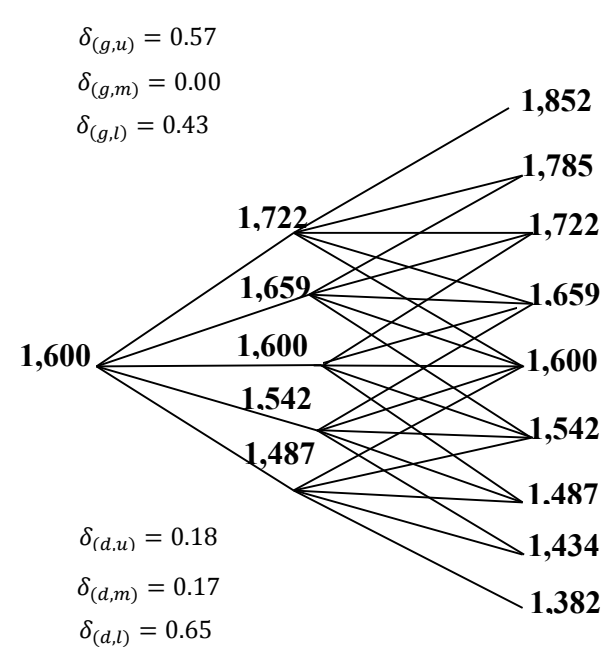

a) Demand distribution within the first 3 time-steps of the lattice model when the mean of the growth regime is equal to 0.15

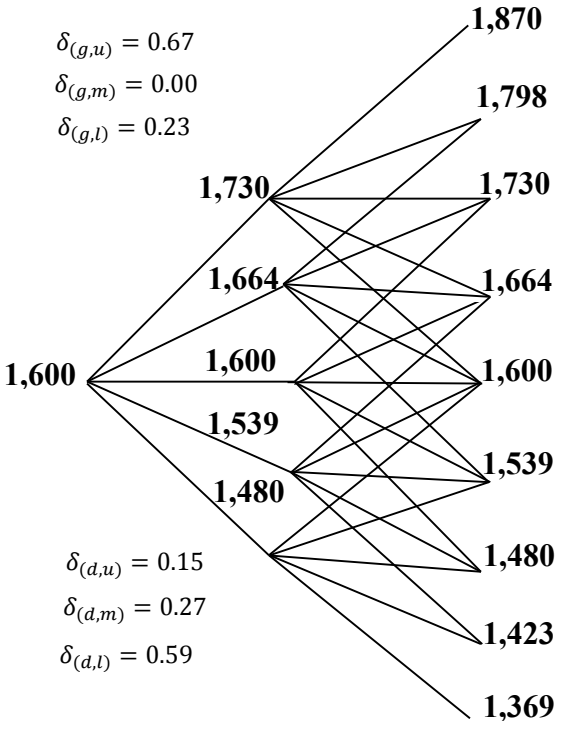

b) Demand distribution within the first 3 time-steps of the lattice model when the mean of the growth regime is equal to 0.35

Figure 7.5: Lattice based demand estimation versus the mean of the growth regime changes

Compared with the model without including HF, the expected total cost has a growth rate between $8.3 \%$ and $0.001 \%$ when $\xi_{g}$ is varied between 0.15 to 0.35 and the life cycle starts with the growth regime. In general, as the expected demand throughout the life cycle increases, the operators working more to satisfy the demand. Therefore, more scrapping and rework units are produced, increasing the difference between the expected cost values with and without HF.

Figure (7.6) demonstrates the effects of $\sigma_{g}$ variation on the performance of the assembly system. The figure also shows the model's outputs with and without HF contribution. The results show that increasing $\sigma_{g}$ causes the total cost to be raised when the life cycle either starts with the growth regime. the total cost changes from $\$ 8,687,622$ to $\$ 9,330,302$ (7.3\% growth) when the volatility of the growth regime increases from 0.15 to 0.35 .

As it is expected, the larger volatility, the larger growth step-size, $\theta_{g}$, in the lattice model. Furthermore, Equations (7.2), (7.3) and (7.4) conclude that the increment of $\sigma_{g}$ from 0.15 to 0.35 causes the demand distribution to include higher values (higher node levels) in the lattice model. For instance, $\delta_{(g, u)}$ changes from 0.40 to 0.58 , respectively. Hence, the expected demand 


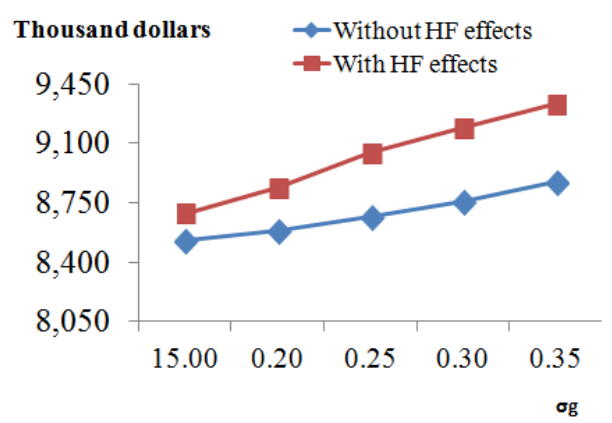

Figure 7.6: The valuation of the total cost versus the volatility of the growth regime changes $\left(B_{j 1}=10\right.$ units $)$

reaches a higher volume. It implies that the life cycle will likely remain in growth for a longer period of time. Therefore, the total cost of the system increases.

Compared with the model without including HF, the expected total cost grows between $1.9 \%$ and $5.2 \%$ when $\sigma_{g}$ is changed from 0.15 to 0.35 and the life cycle starts with the growth regime.

\section{Decay Regime Parameter Variation Effects}

This section examines the assembly line's cost variation versus the mean and volatility of the decay regime changes, shown by Figures $(7.7)$ and (7.8). The results show that increasing the absolute value of $\xi_{d}$ causes the total cost to be reduced when the life cycle starts with the growth regime. Furthermore, compared with the model without HF effects, integrating HF aspects and increasing the absolute value of $\xi_{d}$ estimated a higher expected total cost.

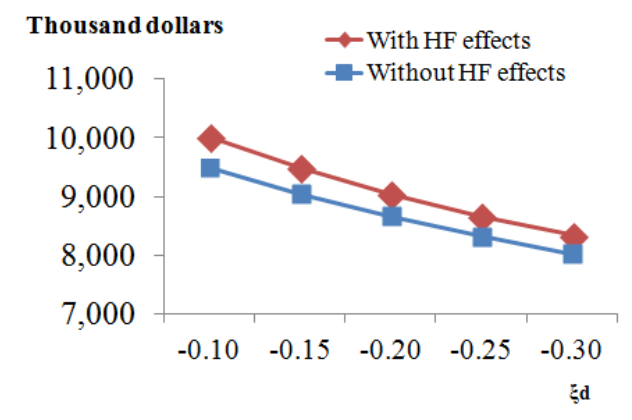

Figure 7.7: The valuation of the total cost versus the mean of the decay regime changes $\left(B_{j 1}\right.$ $=10$ units) 
With the growth regime at the beginning of the product life cycle and including HF effects, the demand has a chance to be in the growth or switch to the decay regime. Hence, the increment of the absolute value of $\xi_{d}$ lets the decay regime branch probabilities of the pentanomial lattice become major factors in estimating the expected demand throughout the life cycle. For instance, $\delta_{(d, u)}, \delta_{(d, m)}$, and $\delta_{(d, l)}$ are respectively equal to $0.20,0.36$, and 0.44 when $\xi_{d}=-0.10$. By changing the mean of the decay regime to -0.30 , the decay regime's branch probabilities are modified to $0.17,0.00$, and 0.83 accordingly. These changes imply that the life cycle have a decreasing tendency to remain in growth for a longer period of time when $\xi_{d}$ varies between -0.10 to -0.30 . Therefore, the total cost reduces from $\$ 10,015,626$ to $\$ 8,340,649$ (17\% cost reduction), accordingly.

Compared with the model without including HF, the expected total cost grows between $5.5 \%$ and $3.4 \%$ when $\xi_{d}$ is changed from -0.10 to -0.30 and the life cycle starts with the growth regime.

Figure (7.8) demonstrates the increment of the total cost versus changing $\sigma_{d}$ from 0.04 to 0.16. The total cost increases from $\$ 9,031,166$ to $\$ 9,224,615$ (2.1\% growth), accordingly. The results imply that by increasing the $\sigma_{d}$, the life cycle has a tendency to include higher demand values. Therefore, the total cost increases. Compared with the model without including HF, the expected total cost grows between $4.6 \%$ to $6.1 \%$ when $\xi_{d}$ is changed between 0.04 and 0.16 .

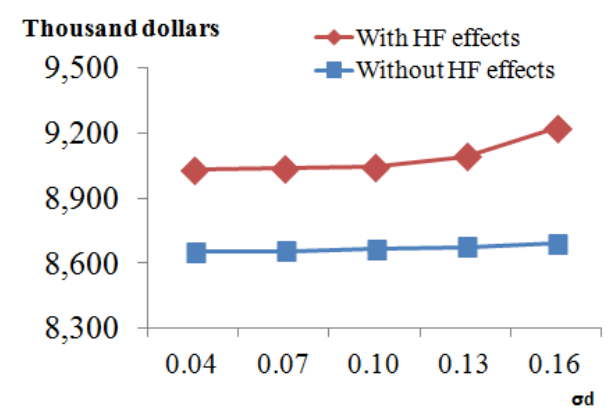

Figure 7.8: Expected total cost versus the volatility of the decay regime (Life cycle starts with the growth regime and $B_{j 1}=10$ units) 


\subsection{Conclusion}

This study presents a novel modeling approach which integrates the effects of work-related human performance variation (internal factor) and demand uncertainty (external factor) into the valuation of the financial performance of manufacturing systems. A three health-state Markov chain has been developed for each operator to make a systematic connection between the HF (ergonomic) aspects of the workplace, the operator's work-related health condition and, consequently, his/her performance variation in terms of health-related productivity and quality losses. By integrating HF-related financial and operational aspects into the cost optimization of an assembly system, it is possible for the model's users to assess the economic impact of the workplace ergonomic condition on the performance of the system.

This study also developed a real option approach to the valuation of HF within the product life cycle. A two-regime product life cycle (growth and decay) was developed to manage the uncertainty involved in the demand value. The demand pursues the stochastic process replicated using a Brownian motion. A pentanomial lattice model was presented to model the stochastic demand within the product life cycle.

The developed model in this research assists managers to better understand how the ergonomic conditions of the workplace affect the behavior of operation systems. In terms of the financial consequences of poor ergonomic design, the results of this study show up to $25.8 \%$ cost increase, when HF aspects of the workplace are included in the system performance modeling. The performance evaluation models without including HF are unable to trace this financial increment at the system level. Thus, their results are underestimated which can distract longterm planning a company from achieving its financial goals. For instance, lets assume that the managers set $30 \%$ as a net profit for the assembly system. According to the scenario 1's results, the increased cost due to the negative effects of WIH risk factors (poor ergonomic design of the workplace) reduces this up to $86 \%$. The traditional performance optimization models usually fail to address this profit reduction. By improving the ergonomic conditions of the workplace operation managers are able to reduce the negative effects of WIH risk factors incurring less expenses. Furthermore, the developed model in this study supports the cost-benefit analysis of different ergonomic solutions in a way that the mangers estimate how much financial benefit (HF-related cost reduction) can be obtained over the product life cycle by reducing WIH risk 
factor exposure levels.

In terms of operational consequences of poor ergonomic design, this study supports operation mangers to include HF considerations in developing the production strategy. For instance, as described in Scenario 1, the expected subcontracting cost changes between $\$ 345,121$ and $\$ 1,127,710$ when the poor ergonomic condition of the assembly system increases the cumulative compression load from 5.5 MNs/shift to $38.8 \mathrm{MNs} /$ shift (demand is in the growth at the beginning of the life cycle). A higher cumulative load causes more health-related productivity and quality losses. Thus, the company cannot completely satisfy the demand and needs subcontractors to cover the unmet demand. The subcontracting cost variation due to the poor ergonomic condition of workplace shows the dependency level of the system's production to the outsource suppliers. This increased dependency can influence the quality and price of the final products. By improving the ergonomic conditions of the assembly system, such as using life assistance or conveyer to move product and materials, the cumulative load effects on the low back of the operators decrease causing, consequently, their work-related productivity and quality to reduce. Hence, fewer products are outsourced. Now, managers can more focus on the assembly system's operational characteristics in developing the production strategy to improve the quality and price of the products.

Taking in account real option approach, the results of scenario 1 demonstrate the dependency of HF effects on the demand regime. For instance, as shown by Figure (7.2), changing the ergonomic design of the workplace causes the cumulative load to increase from 5.5 to 38.8 $\mathrm{MNs} / \mathrm{shift}$. If the life cycle has a tendency to remain in the decay, the cost index changes from 100 to 101.5 due to the cumulative load increase. However, with the growth regime at the beginning of the life cycle, the cost index has a higher value changing from 101.7 to 122.6. These demand regime-related effects imply that with a higher expected demand, the operators are working under higher working loads. Consequently, they have more work-related productivity losses and they produce more defective items incurring the higher cost amount due to the negative effect of poor ergonomic condition. The results of this research also show that a higher necessity for ergonomic improvement solutions when the customer demand is in growth. That is, when the expected demand is higher, the improvement of the HF aspects of the workplace leads the company to save more $\mathrm{HF}$-related expenses.

Testing the stochastic demand's parameters demonstrates the necessary of an accurate es- 
timation for the mean and the volatility of the demand regime. Without estimating accurate information about the distribution of the demand throughout the life cycle the managers are unable to completely predict cost trend changes of the system. For instance, the results in scenario 2 show that changing the volatility of the life cycle regimes modify the expect of cost of the system with the growth regime at the beginning of the product life cycle, shown by Figures (7.6) and (7.8). However, the total cost trend has a higher rate of changing versus the volatility of the growth regime variation. It implies that by increasing $\sigma_{g}$, the life cycle will likely remain in growth for a longer period of time.

The model developed in this chapter is the example of considering the effects of the low back pain WIH risk factors, as a common work-related health problem in manufacturing environment, on the performance of the system over the product life cycle. For future research, the developed generic modeling approach presented in this research can be applied to consider the other important work-related health problem effects such as neck pain, shoulder injuries which can comprise system performance. 


\section{Chapter 8}

\section{Conclusion}

This thesis has developed a modeling framework integrating occupational Human Factors (HF) aspects into Operations Management Performance Optimization (OMPO) models. The framework proposes a hierarchical consideration of occupational HF that includes individual, workstation and system levels. It considers the relationship between the HF elements, which are contributing to employee health, and the performance of operation systems. The developed modeling approach has supported the analytical phases of this $\mathrm{PhD}$ research by providing a road map to a) quantify occupational health effects; b) incorporate occupational HF costs and operational effects into a serial system OMPO models; and c) investigate other operational aspects such as demand uncertainty, inventory and station interactions, when the system performance optimization models include HF aspects.

The first analytical phase of the $\mathrm{PhD}$ research (chapter five) focused on examining the performance optimization of a serial assembly system incorporating Work-related Ill Health (WIH) risk factor effects. A two health-state Markov model was developed to quantify WIH risk factor effects. Then, an optimization model was proposed to minimize the total cost of the serial assembly system. This new optimization model includes employee health-related productivity loss, Occupational Health and Safety (OHS) costs and other operational features of the assembly system such as subcontracting expenses, inventory costs etc. The second phase of the $\mathrm{PhD}$ research (chapter six) has been focused on extending the developed optimization model by including the complexity of employee performance variation in terms of work-related productivity loss and quality defects. This extension improved the proposed modeling approach 
to replicate the negative effects of poor workplace ergonomic design, which would happen in a real system. The third analytical phase of the $\mathrm{PhD}$ research (chapter seven) improved the proposed modeling approach by including stochastic behavior of the demand. In the previous phases, deterministic values were assumed for the customer demand during the given time periods. However, market changes usually impose an uncertain behavior for the demand. For this reason, a growth-decay regime switching model was developed to the valuation of the total cost of the assembly system within a five year product life cycle. The growth regime shows an increasing approach for the demand while the decay regime implies a decreasing behavior. For each regime, the demand follows a stochastic process modeled using a Brownian motion. A pentanomial lattice was developed to include this uncertain demand effect to the valuation of the assembly line performance. To determine the present value of the optimum total cost of the assembly system: first, the total cost was minimized according to the demand value, estimated at each node of the lattice model. This procedure began from the end of the product life cycle. Then, by folding back to the previous time-step, a backward dynamic programing enabled us to estimate the present value of the intermediary nodes. The backward folding continued until the present optimum total cost was determined at the beginning of the life cycle.

\subsection{Challenges, Limitations and Future Research}

Like any novel modeling approach, the proposed models in this $\mathrm{PhD}$ research face challenges and limitations to applying in practice. There can be feasibility challenges in applying the developed modeling approach with regard to the availability of the required in-data and the underlying relations between $\mathrm{HF}$ and operational performance index of an operation system.

The feasibility of obtaining data depends on the scope and context of the operation system to which the developed models will be applied. Many WIH risk factors have been discussed in the existing HF literature including both physical and psychosocial workplace factors and their impacts on disorders for various body parts, as well as on non-localised conditions such as work-depression and coronary heart disease (e.g., Bernard 1997; Karasek et al. 1998; National Research Council 2001; McTernan et al. 2013). Accounting for all WIH hazards is still not feasible in a single model. Furthermore, obtaining some hazard data, such as cumulative spinal loading (e.g. Norman et al. 1998), requires knowledge and the use of modeling software which 
may not be readily available in many firms. Similarly, financial and industrial field data such as production cost per product, the number of products produced in each station, etc. also have to be collected for the workstation and system level analysis. Obtaining this data can be troublesome if the company is, for example, only collecting data in an aggregate form for what is needed.

The other feasibility challenge is related to determining the relationship between operational performance index in the operation system and HF aspects. For example, human-related working quality which is causing defects has been broadly linked to HF aspects in both theoretical and empirical research work. However, the available data is not very deep and does not cover all production contexts and so some judgments, assumptions and estimations must be applied to quantify the connection between human errors and number of defective items, produced in the system. This challenge is shared by other modeling approaches such as business dynamics modeling (Sterman 2000). A further feasibility challenge to the developed modeling approach also lies with the OHS indirect cost items such as overhead costs associated with employee recruitment and replacement. These kinds of indirect cost data have been referred to as "hidden costs" as they are not usually captured in companies' financial databases, and are, therefore, difficult to isolate (e.g., Rose et al. 2013).

In response to these challenges, a number of tactics are proposed that can be applied to generate an accurate estimation (assumption) with regard to HF integration into system performance optimization models. These tactics include: a) examining available research data, b) drawing on available regional statistical data, c) drawing on companies' records, d) applying managerial and employee judgments on critical relationships, and e) making field measurements. In this thesis, the first four tactics were employed in order to justify the assumptions used in the models' analysis and replicate HF effects. The variability in the above assumptions needs further research to develop these models in practice. Fuzzy logic is also a modeling method can be applied in future research to analytically consider the variability of mentioned HF-related assumptions such as HF-related quality loss.

Usability is another challenge that can be faced in applying the developed modeling approach. One important issue in practice is who will use the proposed models? According to the benefits of the modeling approach, ergonomists, operation managers and design engineers are the key target groups who would apply such a model. They can design or improve systems 
while replicating the dynamic behavior of the systems with regard to HF effects. The ability to examine long-run performance may also be of interest to top managers who could employ the modeling approach to help meet more long term strategic goals. The proposed models can also support senior decision makers in understanding the real impact of employees' work-related health effects on the long-term performance of systems - a capability they do not currently have. For each potential user, there is a set of requirements in terms of production volume, working quality and cost items to consider their needs and capabilities for generating in-data for the models and interpreting their outputs in a good way. Due to the ability of the modeling approach to conform with the users' requirements, the developed models should be customized in terms of the available HF and non- HF data for the model inputs and outputs. It is likely that, like HF-economic tools, a team approach would be necessary to develop and apply such models to real business planning work (e.g., Rose et al. 2013).

According to the purpose of this research, the developed models are also limited to employees' work-related performance variation. This focus supported our analysis to comprehensively evaluate the effect of $\mathrm{HF}$ aspects on optimizing the performance of a system. In general, similar to humans, machine failure can also interrupt the operational activities of the system. Including the performance variation of operators and machines may result in replicating the system's behavior in a more realistic way. Therefore, further research is needed to integrate the contribution of machine and human performance variations in evaluating the performance of systems.

In this research, the developed models were also examined using HF-information of low back pain due to: a) the importance of work-related low back pain as a major occupational health problem in manufacturing environment, and b) the application of a solid empiricalbased primary HF information of a research in automotive company in Canada (Kerr 1997). These empirical data sets allow us to evaluate the models through replicating HF effects, which are happening in a real working environment. In future, depending on working conditions in industry and service sectors, the proposed modeling approach has a capability to consider other possible work-related health problem effects such as neck pain, shoulder injuries etc.

Further limitation in the developed models regards the number of stations, workers, and inventory levels. In this study, four workstations, four workers, with four buffers are considered in modeling the serial assembly system. Enlarging the operation system may result in 
a more complicated system modeling and a longer time of computation for numerical testing. Particularly, as used in chapter 7, a dynamic programing model was developed to replicate the stochastic behavior of the demand. When the optimum cost at each node of the pentanomial lattice is estimated, the developed model brings nine results regrading different inventory level combinations. Determining the minimum cost taking into account the inventory level combinations results in estimating the expected cost at each node of the lattice. With regard to 7,140 nodes for a 5 year product life cycle, significant computation time is needed to the valuation of the total cost, completed in the previous chapter numerical analysis. Hence, increasing the number of stations or number of workers, or buffer levels will make the computation and system modeling very complex and will take a long time to analyze the output of the models. In the future, the models can be expanded to include the above limitations by writing programs which can handle the increased complex computations.

The developed models of this study can also be extended by considering the productionrelated learning and forgetting of humans (competency levels). The learning and forgetting of humans have interesting subjects to many researches in order to investigate their associated

effects on the production variability of employees at work (e.g., Omar et al. 2011; Wahab and Jaber 2010). In general, the longer the period of production, the more the productivity increases. Moreover, the reduction of human errors causes few defective items to produce due to a higher competency (experience) level of the employees. However, the longer the stoppage in working such as temporarily absent from the work due to occupational health problems causes the greater forgetting effect of human resulting a lower production rate and a higher chance of human errors. By extending the developed models of this study to include humans competency variation due to learning and forgetting patterns, it is possible for future research to better replicate the effects of employee performance variations due to working condition in optimizing the performance of operation systems.

\subsection{Significance of the Developed Modeling Approach}

Apart from the challenges and limitations of the proposed models in this thesis, the developed modeling approach demonstrated the importance of HF considerations in optimizing the performance of serial systems. The results of this research also indicated negative operational 
and financial consequences of a poor HF design of working environments. System performance optimization models without including HF are unable to track these effects.

In chapter five, an analytical model was developed to capture the transition probability from the healthy state to the work-related pain/injury state of employees taking into account the given exposure levels of WIH risk factors. Then, according to the concept of Logistic regression, a two health-state Markov model was proposed to quantify WIH risk factor effects (ergonomic condition of the workplace) on employee health-state in a probabilistic way. Ultimately, an optimization model was developed to minimize the total cost of an assembly system with regard to employee health-related productivity loss. From an ergonomics point of view, for the first time, both direct and indirect OHS costs were incorporated when the performance of the operation system was optimized. The developed model was numerically tested to evaluate the effects of (HF) ergonomic design changes on the performance of an operation system. The numerical results have indicated that there is between $0.5 \%$ and $8 \%$ difference in the total cost of the system with and without including HF effects in the performance optimization model. The results also showed that by improving the ergonomic design of the workplace, the assembly system's production efficiency is enhanced causing few products to be outsourced. The traditional OMPO models avoiding HF effects are unable to isolate the effects of improving ergonomic design of the workplace in developing outsourcing strategies for companies.

In chapter six, a three health-state Markov chain was developed for each operator of the assembly system to make a connection between HF (ergonomic) aspects of the workplace and the employee's work-related productivity and quality variations. According to the failure function concept, the developed modeling approach enabled us to quantify the effect of HF aspects of the working environment design on human errors and working quality. Ultimately, integer programming method was used to optimize the total cost of the assembly system with regard to operational and financial consequences of HF changes. The numerical analysis indicated that the expected human error rate increased from $0.03 \%$ to $5.7 \%$ due to varying low back pain physical risk factor exposures (deteriorating the ergonomic condition of the workplace). This error rate reached a higher level, up to $6.5 \%$, when the psychosocial risk factor exposure changes were also considered. The results of this study also showed that failure to include HF effects has led to an underestimated total cost of the system. Compared to the optimization model without including HF effects, the minimized total cost of the assembly line increased between 
$0.02 \%$ and $32 \%$ when both employee productivity and quality losses due to poor HF design of the workplace were integrated into the optimization model. The analytical results concluded that having a safe working environment results in least negative effects of working conditions on the health and operational characteristics of the employees of the assembly system. Hence, a minimum cost difference $(0.02 \%)$ between the models with and without including HF effects is estimated. However, by exacerbating HF (ergonomic) design of the workplace, the new working conditions increases the chance of developing work-related low back pain. Subsequently, the efficiency of the operation system is a due to increasing employee HF-related productivity and quality losses.

In chapter seven, the optimization model was proposed by including the stochastic behavior of demand. A pentanomial lattice model was developed to replicate the growth-regime switching model of the demand. The new developed modeling approach was numerically tested with two scenarios.

The first scenario followed the real options approach in the presence of HF variations. With respect to a specified pattern for the demand's growth and decay regimes, the valuation of the total cost was accomplished by including the effects of HF-related employee productivity and errors variations. The model was numerically tested by changing physical and psychosocial WIH risk factors (changing workplace ergonomic condition). The results were compared with a value option model without including WIH effects. Taking into account physical risk factor exposure changes, there is between $1.07 \%$ and $22 \%$ cost difference for the models with and without considering HF effects when the life cycle begins with the growth regime. With regard to psychosocial risk factor exposure changes, the associated cost difference varies between $0.1 \%$ and $25.8 \%$.

In the second scenario, the value option model robustness was examined. The mean and volatility of the growth and decay regimes were changed to assess the total cost of the operation system versus changing the stochastic behavior of the demand. The results indicated the sensitivity of the cost of the system to demand distribution within the product life cycle. Hence, it is necessary to accurately estimate the parameters of the regimes, specifying the demand distribution throughout the life cycle. Without predicting a demand behavior, the cost is over or underestimated.

Taking into account the models' analysis, this $\mathrm{PhD}$ research creates a new evaluation ap- 
proach that supports managers and engineers to better understand the consequences of working environment conditions on both employee well-being and performance, which subsequently affect the financial performance of operation systems. Furthermore, integrating HF effects into performance evaluation models enables operation engineers and scholars to optimize the performance of system with regard to a more realistic replication of the systems' behavior. The implementation of the proposed modeling approach in practice will also help practitioners find out how the performance of their systems will be enhanced by investing in safer working conditions for employees. 


\section{Appendix 1}

\section{Psychosocial measurement methods}

The examples of questions measured LBP psychosocial risk factor exposures are presented in the following. These questions were borrowed from psychosocial measurement methods, developed by Perlin and Schooler (1978); Borg (1982); Karasek et al. (1998); Jencks et al. (1998). The component questions are usually measured with Likert-type scale, with reply options of either "Often, Sometimes, Rarely or Never" or "Strongly Agree, Agree, Disagree, Strongly Disagree". The Borg perceived exertion measurement components are scaled from 0 to 14 .

\section{Component questions of the Social environment:}

There is not a pleasant atmosphere at my workplace.

There is nobody at work that I can talk to about job problems.

There are frequent conflicts at my workplace.

I feel I am harassed at work because of my race or sex.

\section{Component questions of the Job control:}

My job requires that I learn new things.

My job involves a lot of repetitive work.

My job requires me to be creative.

My job allows me to make a lot of decisions on my own.

I have a lot of say about what happens on my job.

I have an opportunity to develop my own special abilities. 
I get to do a variety of different things on my job.

Do you need permission or do you have to find someone to take your place if you leave your work area for five or ten minutes?

How much do workers take part in decision making in the workplace?

I feel employees ideas/options are listed to by management.

I can influence the way work is organized in my work area.

I have control over how my work area is set up.

I have little control over the things that happen to me.

What happens to me in the future mostly depends on me.

I often feel helpless in dealing with problems at work.

There is no way I can solve some of the problems that I have at work.

On my Job, I have freedom to decide how I do my work.

\section{Physical exertion (using Borg Scale):}

0

1 very, very light.

2

3 very light.

4

5 usually light.

6

7 a bit demanding.

8

9 demanding.

10

11 very demanding.

12

13 very, very demanding. 


\section{References}

Abrahamsson, L. (2000). Production economics analysis of investment initiated to improve working environment. Applied Ergonomics, 31(1):1-7.

Ahmed, S., King, A. J., and Parija, G. (2003). A multi-stage stochastic integer programming approach for capacity expansion under uncertainty. Journal of Global Optimization, 26(1):324.

Ahmed, S. and Sahinidis, N. (2008). Selection, acquisition, and allocation of manufacturing technology in a multi-period environment. European Journal of Operational Research, $187: 807-821$.

Ahsberg, E. (1998). Perceived Fatigue related to Work. PhD thesis, University of Stockholm.

Aitkena, J., Childerhouse, P., and Towill, D. (2003). The impact of product life cycle on supply chain strategy. International Journal of Production Economics, 85:127-140.

Al-e hashem, S. M., Baboli, A., and Sazvar, Z. (2013). A stochastic aggregate production planning model in a green supply chain: Considering flexible lead times, nonlinear purchase and shortage cost functions. European Journal of Operational Research, 230:26-41.

Alexopoulos, E. C., Eleni, C. K., Bakoyannis, G., Tanagra, D., and Burdorf, A. (2008). Risk factors for sickness absence due to low back pain and prognostic factors for return to work. European Spine Journal, 17:1185-1192.

Allen, H., Hubbard, D., and Sullivan, S. (2005). The burden of pain on employee health and productivity at a major provider of business services. Journal of Occupational and Environmental Medicine, 47:658-670. 
Andersson, R. (1992). Economic evaluation of ergonomic solutions: Part ii- the scientific basis. Journal of Industrial Ergonomics, 10(1-2):173-178.

Armenian, H. K. (2009). The case-control method. Oxford University Press.

Arnetz, B., Sjugren, B., and Rydehn, B. (2003). Early workplace intervention for employees with musculoskeletal-related absenteeism: A prospective controlled intervention study. Journal of Occupational and Environmental Medicine, 45(5):499-506.

AWCBC (2004). Canadian work injuries and diseases. Technical report, Association of Workers Compensation Boards of Canada.

Axelsson, J. (2000). Quality and ergonomics: Towards successful integration. PhD thesis, University of Linkoping.

Azzalini, A. (1994). Logistic regression and other discrete data models for serially correlated observations. Journal of the Italian Statistical Society, 3(2):169-179.

Baldwin, M. L. (2004). Reducing the costs of work-related musculoskeletal disorders: Targeting strategies to chronic disability cases. Electromyography and Kinesiology, 14(1):33-41.

Barbarosoglu, G. and Ozdamar, L. (2000). Analysis of solution space-dependent performance of simulated annealing: The case of the multi-level capacitated lot sizing problem. Computers and Operations Research, 27:895-903.

Bard, J., Morton, D., and Wang, Y. (2007). Workforce planning at usps mail processing and distribution centers using stochastic optimization. Annals of operations Research, 155(1):5178.

Barker, L. and Nussbaum, M. A. (2011). Fatigue, performance and the work environment: a survey of registered nurses. Journal of Advanced Nursing, 67(6):1370-1382.

Beevis, D. (2003). Ergonomics costs and benefits revisited. Applied Ergonomics, 34(5):491-496.

Bergstrom, G., Bodin, L., Hagberg, J., Aronsson, G., and Josephson, M. (2009). Sickness presenteeism today, sickness absenteeism tomorrow? A prospective study on sickness presenteeism and future sickness absenteeism. Journal of Occupational and Environmental Medicine, 51(6):629-638. 
Bernard, M. (1997). Musculoskeletal disorders and workplace factors: A critical review of the epidemiologic evidence for work-related musculoskeletal disorders of the neck, upper extremity, and low back. Technical report, US Department of Health and Human Services, National Institute for Occupational Safety and Health.

Berretta, R. and Rodrigues, L. F. (2004). A memetic algorithm for a multistage capacitated lot-sizing problem. International Journal of Production Economics, 87(1):67-81.

Betterton, C. and Silver, S. (2012). Detecting bottlenecks in serial production lines: A focus on interdeparture time variance. International Journal of Production Research, 50(15):41584174 .

Bevilacqua, M., Emanuele Ciarapica, F., and Mazzuto, G. (2012). Analysis of injury events with fuzzy cognitive maps. Journal of Loss Prevention in the Process Industries, 25(4):677-685.

Bhattacharya, M., Gibson, D., and Doty, D. (2005). The effects of flexibility in employee skills, employee behaviors and human resource practices on firm performance. Journal of Management, 31(4):622-640.

Bhattacharye, D. (2005). Production, manufacturing and logistics. European Journal of Operational Research, 162:786-791.

Birdi, K., Clegg, C., Patterson, M., Robinson, A., Stride, C., Wall, T., and Wood, S. (2008). The impact of human resource and operational management practices on company productivity: A longitudinal study. Personnel Psychology, 61(3):467-501.

Bollen, N. P. (1999). Real options and product life cycles. Management Science, 45(5):670-684.

Bollen, N. P., Gray, S. F., and Whaley, R. E. (2000). Regime switching in foreign exchange rates: Evidence from currency option prices. Journal of Econometrics, 94:239-276.

Bongers, P. M., Kremer, A. M., and Laak, J. t. (2002). Are psychosocial factors, risk factors for symptoms and signs of the shoulder, elbow, or hand/wrist?: A review of the epidemiological literature. American Journal of Industrial Medicine, 41(5):315-342.

Borg, G. (1982). Psychosocial bases of perceived exertion. Medicine and Science in Sports and Exercise, 14(5):377-381. 
Boudreau, J., Hopp, W., McLain, J., and Thomas, L. J. (2003). On the interface between operations management and human resources management. Manufacturing and Service Operations Management, 5(3):179-202.

Bowman, G. R., Huang, X., and Pande, V. S. (2010). Network models for molecular kinetics and their initial applications to human health. Cell Research, 20(6):622-630.

Boysen, N., Fliedner, M., and Scholl, A. (2008). Assembly line balancing: Which model to use when? International Journal of Production Economics, 111(2):509-528.

Brahimi, N., Dauzere-Peres, S., Najid, N., and Nordli, A. (2006). Single item lot sizing problems. European Journal of Operational Research, 168(1):1-16.

Bravo, M. and Gonzalez, I. (2009). Applying stochastic goal programming: A case study on water use planning. European Journal of Operational Research, 196:1123-1129.

Brown, G. and Baker, J. (2004). Work-related musculoskeletal disorders in sonographers. Journal of Diagnostic Medical Sonography, 20(2):85-93.

Bubb, H. (2005). Human reliability: A key to improved quality in manufacturing. Human Factors and Ergonomics in Manufacturing, 15:353-368.

Buckle, P. W. and Devereux, J. J. (2002). The nature of work-related neck and upper limb musculoskeletal disorders. Applied Ergonomics, 33(3):207-217.

Bultmann, U., Franche, R.-L., Hogg-Johnson, S., Lee, H., Severin, C., Vidmar, M., and Carnide, N. (2007). Health status, work limitations, and return-to-work trajectories in injured workers with musculoskeletal disorders. Quality of Life Research, 16(7):1167-1178.

Burton, W. N., Chen, C.-Y., Conti, D. J., Schultz, A. B., Pransky, G., and Edington, D. W. (2005). The association of health risks with on-the-job productivity. Journal of Occupational and Environmental Medicine, 47(8):769-777.

Butler, R. J., Baldwin, M. L., and Johnson, W. G. (2006). The effects of occupational injuries after returns to work: Work absences and losses of on-the-job productivity. Journal of Risk and Insurance, 73(2):309-334. 
Buxey, G. (2005). Aggregate planning for seasonal demand: Reconciling theory with practice. International Journal of Operations and Production Management, 25(11):1083-1100.

Campo, M. and Darragh, A. R. (2012). Work-related musculoskeletal disorders are associated with impaired presenteeism in allied health care professionals. Journal of Occupational and Environmental Medicine, 54:64-70.

Carayon, P., Smith, M., and Haims, M. (1999). Work organization, job stress and work-related musculoskeletal disorders. Human Factors, 41:644-663.

Cattrysse, D., Salomon, M., Kuik, R., and Van Wassenhove, L. (1993). A dual ascent and column generation heuristic for the discrete lotsizing and scheduling problem with setup times. Management Science, 39(4):477-486.

Chaffin, D., Andersson, G. B. I., and Martin, B. J. (2006). Occupational Biomechanics. New York, NY: John Wiley \& Sons.

Chen, Z. and Forsyth, P. A. (2010). Implications of a regime-switching model on natural gas storage valuation and optimal operation. Quantitative Finance, 10(2):159-176.

Chenevert, D. and Tremblaya, M. (2009). Fits in strategic human resource management and methodological challenge: empirical evidence of influence of empowerment and compensation practices on human resource performance in canadian firms. The International Journal of Human Resource Management, 20(4):738-770.

Chiu, H.-N., Lee, C.-T., Huang, D.-K., and Chiu, W.-H. (2012). A fuzzy multi-objective supply chain production planning model: A case of the network product manufacturing industry in taiwan. In Service Systems and Service Management (ICSSSM), 2012 9th International Conference on, pages 191-196.

Choi, B., Levitsky, M., Lloyd, R., and Stones, I. (1996). Patterns and risk factors for sprains and strains in ontario, canada 1990: An analysis of the workplace health and safety agency data base. Journal of Occupational and Environmental Medicine, 38(4):379-389.

Coenen, P., Kingma, I., Boot, C., Twisk, J., Bongers, P., and Diean, J. (2013). Cumulative low back load at work as a risk factor of low back pain: A prospective cohort study. Journal of Occupational Rehabilitation, 23(1):11-18. 
Coenen, P., Kingma, I., Boot, C. R., Douwes, M., Bongers, P. M., and van Diean, J. H. (2012). Work-site musculoskeletal pain risk estimates by trained observers: A prospective cohort study. Ergonomics, 55(11):1373-1381.

Cole, D. C. and Rivilis, I. (2004). Individual factors and musculoskeletal disorders: a framework for their consideration. Journal of Electromyography and Kinesiology, 14(1):121-127.

Cote, P., Kristman, V., Vidmar, M., Van Eerd, D., Hogg-Johnson, S., Beaton, D., and Smith, P. (2009). The prevalence and incidence of work absenteeism involving neck pain: A cohort of ontario lost-time claimants. Journal of Manipulative and Physiological Therapeutics, 32:219226.

Courtney, T. and Webster, B. (1999). Disabling occupational morbidity in the united states: An alternative way of seeing the bureau of labour statistics' data. Journal of Occupational and Environmental Medicine, 41(1):60-69.

Cox, J. C., Ross, J., and Rubinstein, M. (1979). Option pricing: A simplified approach. Journal of Financial Economics, 7:229-263.

Cox, L. A. and Popken, D. A. (2004). Quantifying human health risks from virginiamycin used in chickens. Risk Analysis, 24(1):271-288.

Coyte, P. C., Asche, C. V., Croxford, R., and Chan, B. (1998). The economic cost of musculoskeletal disorders in canada. Arthritis $\&$ Rheumatism, 11:315-325.

Da Costa, B. R. and Vieira, E. R. (2009). Risk factors for work-related musculoskeletal disorders: A systematic review of recent longitudinal studies. American Journal of Industrial Medicine, $53(3): 285-323$.

Da Silva, C. G., Figueira, J., Lisboa, J., and Barman, S. (2006). An interactive decision support system for an aggregate production planning model based on multiple criteria mixed integer linear programming. Omega, 34(2):167-177.

Dagenais, S., Caro, J., and Haldeman, S. (2008). A systematic review of low back pain cost of illness studies in the united states and internationally. The spine Journal, 8:8-20. 
Das, T. and Teng, B.-S. (2000). A resource-based theory of strategic alliances. Journal of Management, 26(1):31-61.

David, C. (2005). Ergonomic methods for assessing exposure to risk factors for work-related musculoskeletal disorders. Occupational Medicine, 55:190-199.

Dawson, A. P., Steele, E. J., Hodges, P. W., and Stewart, S. (2009). Development and the reliability of an extended version of the nordic musculoskeletal questionnaire (nmq-e): A screening instrument for musculoskeletal pain. The Journal of Pain, 10(5):517-526.

De Menezes, L., Wood, S., and Gelade, G. (2010). Ahe integration of human resource and operation management practices and its link with performance: A longitudinal latent class study. Journal of Operations Management, 28(6):455-471.

Dellaert, N. and Jeunet, J. (2000). Solving large unconstrained multilevel lot-sizing problems using a hybrid genetic algorithm. International Journal of Production Research, 38(5):10831099.

Denis, D., St-Vincent, M., Imbeau, D., Jetta, C., and Nastasia, I. (2008). Intervention practices in musculoskeletal disorder prevention: A critical literature review. Applied Ergonomics, $39(1): 1-14$.

Desai, P., Koenigsberg, O., and Purohit, D. (2007). The role of production lead time and demand uncertainty in marketing durable goods. Management Science, 53(1):150-158.

Deuffic-Burban, S., Poynard, T., Sulkowski, M., and Wong, J. (2007). Estimating the future health burden of chronic hepatitis c and human immunodeficiency virus infections in the United States. Journal of Viral Hepatitis, 14(2):107-115.

Devereux, J., Buckle, P., and Vlachonokilis, I. (1999). Interactions between physical and psychosocial risk factors at work increase the risk of back disorders: An epidemiological approach. Journal of Occupational and Environmental Medicine, 56:343-353.

Devereux, J., Vlachonikolis, I., and Buckle, P. (2002). Epidemiological study to investigate potential interaction between physical and psychosocial factors at work that may increase the risk of symptoms of musculoskeletal disorder of the neck and upper limb. Occupational and Environmental Medicine, 59(4):269-277. 
Dode, P. (2011). The integration of human factors into discrete event simulation and technology acceptance in engineering design. Master's thesis, Ryerson University.

Driessen, M., Bosmans, J., Proper, k., Anema, J., Bongers, P., and Van der Beek, A. (2012). The economic evaluation of a participatory ergonomics programme to prevent low back and neck pain. Journal of Prevention, Assessment and Rehabilitation, 41:2135-2320.

Driessen, M., Proper, K. I., Anema, J., Knol, D. L., Bongers, P. M., and van der Beek, A. J. (2010). Participatory ergonomics to reduce exposure to psychosocial and physical risk factors for low back pain and neck pain: Results of a cluster randomised controlled trial. Journal of Occupational and Environmental Medicine, 68:674-681.

Drury, C. G. (2000). Global quality: Linking ergonomics and production. International Journal of Production Research, 38(17):4007-4018.

Dul, J., Bruder, R., Buckle, P., Carayond, P., Falzone, P., Marrasf, J. W., Wilsong, J. R., and Van doelenh, B. (2012). A strategy for human factors/ergonomics: Developing the discipline and profession. Ergonomics, 55(4):377-395.

Dul, J. and Neumann, W. P. (2009). Ergonomics contributions to company strategies. Applied Ergonomics, 40(4):745-752.

Eklund, J. (1997). Ergonomics, quality and continuous improvementconceptual and empirical relationships in an industrial context. Ergonomics, 40(10):982-1001.

Eklund, J. (2000). Development work for quality and ergonomics. Applied Ergonomics, $31(6): 641-648$.

El-Maraghy, W. H., Nada, O. N., and El-Maraghy, H. A. (2008). Quality prediction for reconfigurable manufacturing systems via human error modelling. International Journal of Computer Integrated Manufacturing, 21(5):584-598.

Elias, R. S., Wahab, M. I. M., and Fang, L. (2014). Comparison of regime-switching temperature modeling approaches for applications in weather derivatives. European Journal of Operational Research, 232(3):549-560. 
Eltayeb, S., Staal, J. B., Kennes, J., Lamberts, P. H., and de Bie, R. A. (2007). Prevalence of complaints of arm, neck and shoulder among computer office workers and psychometric evaluation of a risk factor questionnaire. BMC Musculoskeletal Disorders, 68(8):1-11.

Erdinc, O. and Vayvay, O. (2008). Quality improvement through ergonomics methodology: conceptual framework and an application. International Journal of Productivity and Quality Management, 3(3):311-324.

Erdinc, O. and Yeow, P. H. (2011). Proving external validity of ergonomics and quality relationship through review of real-world case studies. International Journal of Production Research, 49(4):949-962.

Errico, A., Viotti, S. snd Baratti, A., Mottura, B., Barocelli, A., Tagna, M., Sgabelluri, B. Battaglino, P., and Converso, D. (2013). Low back pain and associated presenteeism among hospital nursing staff. Journal of Occupational Health, 55:276-283.

Escorpizo, R. (2008). Understanding work productivity and its application to work-related musculoskeletal disorders. International Journal of Industrial Ergonomics, (38):291-297.

EUOSHA (2001). European agency for health and safety at work, economic impact of occupational safety and health in the member states of the european union.

EUOSHA (2008). European agency for safety and health at work, work-related musculoskeletal disorders: Prevention report.

EUOSHA (2011). European agency for safety and health at work, musculoskeletal disorders: General questions.

Fagarasanu, M. and Kumar, S. (2003). Carpal tunnel syndrome due to keyboarding and mouse tasks: a review. International Journal of Industrial Ergonomics, 31(2):119-136.

Ferguson, S. A., Marras, W. S., and Burr, D. L. (2005). Differences among outcome measures in occupational low back pain. Journal of Occupational Rehabilitation, 15(3):329-341.

Ferguson, T. (2002). Linear programming: Concise introduction. Emerald. 
Ferreira, D., Morabito, R., and Rangel, S. (2009). Solution approaches for the soft drink integrated production lot sizing and scheduling problem. European Journal of Operational Research, 196(2):697-706.

Feuerstein, M., Shaw, W. S., Nicholas, R., and Huang, G. D. (2004). From confounders to suspected risk factors: Psychosocial factors and work-related upper extremity disorders. Electromyography and Kinesiology, (14):171-178.

Fontes, D. B. (2008). Fixed versus flexible production systems: A real options analysis. European Journal of Operational Research, 188:169-184.

Freivalds, A. and Niebel, B. (2009). Niebel's Methods, Standards and Work Design. McGraw Hill.

Fung, R., Tang, J., and Wang, D. (2003). Multiproduct aggregate production planning with fuzzy demands and fuzzy capacities. Systems, Man and Cybernetics, Part A, IEEE Transactions on, 33(3):302-313.

Gademann, N. and Schutten, M. (2005). Linear-programming-based heuristics for project capacity planning. IIE Transactions, 37(2):153-165.

Garg, A., Boda, S., Hegmann, K. T., Moore, J. S., Kapellusch, J. M., Bhoyar, P., Thiese, M., Merryweather, A., Deckow-Schaefer, G., Bloswick, D., and Malloy, E. J. (2013). The niosh lifting equation and low-back pain, part 1: Association with low-back pain in the backworks prospective cohort study. Human Factors: The Journal of the Human Factors and Ergonomics Society, 56(1):6-28.

Giuntini, R. E. (2000). Mathematical characterization of human reliability for multi-task system operations. Paper presented at the Systems, Man, and Cybernetics, 2000 IEEE International Conference, pages 25-35.

Glenn, N. D. (2005). Cohort analysis. Thousand Oaks, Calif.: Sage Publications.

Goetzel, R., Long, S., Ozminkowski, R., Hawkins, K., Wang, S., and Lynch, W. (2004). Health, absence, disability, and presenteeism cost estimates of certain physical and mental health conditions affecting u.s. employers. Journal of Occupational and Environmental Medicine, $46(4): 398-412$. 
Goggins, R. W., Spielholz, P., and Nothstein, G. L. (2008). Estimating the effectiveness of ergonomics interventions through case studies: Implications for predictive cost-benefit analysis. Journal of Safety Research, 39(3):339-344.

Gold, J., Errico, A., Katz, J., Gore, R., and Punnett, L. (2009). Specific and non-specific upper extremity musculoskeletal disorder syndromes in automobile manufacturing workers. American Journal of Industrial Medicine, 52(2):124-132.

Gonzalez, B. A., Belarmino, A.-D., and Pilar, G. T. (2003). Ergonomic performance and quality relationship: an empirical evidence case. International Journal of Industrial Ergonomics, pages $33-40$.

Gopalakrishnan, M., Ding, K., Bourjolly, J., and Mohan, S. (2001). A tabu-search heuristic for the capacitated lot-sizing problem with set-up carryover. Management Science, 47(6):851863.

Gua, J., Goetschalck, M., and McGinnis, L. F. (2010). Research on warehouse design and performance evaluation: A comprehensive review. European Journal of Operational Research, 203:539-549.

Hallgren, M. and Olhager, J. (2009). Flexibility configurations: Empirical analysis of volume and product mix flexibility. Omega, 37(4):746-756.

Ham, D.-H., Park, J., and Jung, W. (2012). Model-based identification and use of task complexity factors of human integrated systems. Reliability Engineering E5 System Safety, 100:33-47.

Hatoum, H. T., Keye, W. R., Marrs, R. P., Walton, S. M., and Marshall, D. C. (2005). A markov model of the cost-effectiveness of human-derived follicle-stimulating hormone (fsh) versus recombinant fsh using comparative clinical trial data. Fertility and Sterility, 83(3):804807.

Haukka, E. (2010). Musculoskeletal disorders and psychosocial factors at work Effects of a participatory ergonomics intervention in a cluster randomized controlled trial. $\mathrm{PhD}$ thesis, Faculty of Medicine of the University of Helsinki. 
Helander, M. G. and Burri, G. J. (1995). Cost effectiveness of ergonomics and quality improvements in electronics manufacturing. International Journal of Industrial Ergonomics, $15: 137-151$.

Hendrick, H. W. (2003). Determining the cost and benefits of ergonomics projects and factors that lead to their success. Applied Ergonomics, 34(5):419-427.

Heneweer, H., Staes, F., Aufdemkampe, G., Rijn, M., and Vanhees, L. (2011). Physical activity and low back pain: A systematic review of recent literature. European Spine Journal, 20(6):826-845.

Heuvel, S., Geuskens, G., Hooftman, W., Koppes, L., and Bossche, S. (2010). Productivity loss at work; health-related and work-related factors. Journal of Occupational Rehabilitation, 20(3):331-339.

Hosmer, D. and Lemeshow, S. (2000). Applied logistic regression. Second edition, John Wiley and Sons INC.

Howard, K. J., Mayer, T. G., and Gatchel, R. J. (2009). Effects of presenteeism in chronic occupational musculoskeletal disorders: Stay at work is validated. Journal of Occupational and Environmental Medicine:, 51:724-731.

Hoy, D., Brooks, P., Blyth, F., and Buchbinder, R. (2010). The epidemiology of low back pain. Best Practice 83 Research Clinical Rheumatology, 24(6):69-781.

HRSDC (2010). Occupational injuries and diseases in canada, 1996-2008 injury rates and cost to the economy. Technical report, Occupational Health and Safety Division, Labour Program, Human Resources and Skills Development Canada.

Huang, K. and Ahmed, K. (2010). A stochastic programming approach for planning horizons of infinite horizon capacity planning problems. European Journal of Operational Research, 200(1):74-84.

Huisman, R. and Mahieu, R. (2003). Regime jumps in electricity prices. Energy Economics, 25(5):425-434. 
Hung, S., Chen, Y., Shih, C., and Hung, M. (2003). Using tabu search with ranking candidate list to solve production planning problems with setups. Computers and Industrial Engineering, 45(4):615-634.

Hung, Y. F. and Chien, K. L. (2000). A multi-class multi-level capacitated lot sizing model. Journal of the Operational Research Society, 51:1309-1318.

IEA-Council (2000). The discipline of ergonomics. international ergonomics society, 1 Imbeau, d., Bellemare, m., Courville, j., Bergeron, s., Desjardins, l., (2001) Ergonomics in a design environment. In: Karwowski,w., (ed.). International Encyclopedia of Ergonomics and Human Factors, 1 .

Iridiastadi, H. and Nussbaum, M. A. (2006). Muscular fatigue and endurance during intermittent static efforts: Effects of contraction level, duty cycle, and cycle time. Journal of the Human Factors and Ergonomics Society, 48(4):710-720.

Iverson, D., Lewis, K., Caputi, P., and Knospe, S. (2010). The cumulative impact and associated costs of multiple health conditions on employee productivity. Journal of Occupational and Environmental Medicine, 52(12):1206-1211.

Iwasea, M. and Ohnob, K. (2011). The performance evaluation of a multi-stage jit production system with stochastic demand and production capacities. European Journal of Operational Research, 214:216-222.

Jaber, M. and Sikstrom, S. (2004). A note on "an empirical comparison of forgetting models". IEEE Transactions on, Engineering Management, 51(2):233-234.

Jaber, M. Y., Bonney, M., and Moualek, I. (2009). Lot sizing with learning, forgetting and entropy cost. International Journal of Production Economics, 118(1):19-25.

Jaber, M. Y., Givi, Z., and Neumann, W. P. (2013). Incorporating human fatigue and recovery into the learning-forgetting process. Applied Mathematical Modelling, 37(13):7287Ü7299.

Jaber, M. Y. and Guiffrida, A. L. (2007). Observations on the economic manufacture quantity model with learning and forgetting. International Transactions in Operational Research, 14(2):91-104. 
Jaber, M. Y. and Khan, M. (2010). Managing yield by lot splitting in a serial production line with learning, rework and scrap. International Journal of Production Economics, 124(1):3239.

Jallon, R., Imbeau, D., and Marcellis-Warin, N. (2011). Development of an indirect-cost calculation model suitable for workplace use. Journal of Safety Research, 42:149-164.

Jencks, C., Perman, L., and Rainwater, L. (1998). What is a good job? A new measure of labour market success. American Journal of Sociology, 93(6):1322-1357.

Jenkins, S. and Rickards, J. (2001). The economics of ergonomics: three workplace design case studies, in Alexander, D.C. and Rabourn, R. (Eds). Taylor \& Francis, London.

Ji, Q., Lan, P., and Looney, C. (2006). A probabilistic framework for modeling and real-time monitoring human fatigue. IEEE transactions on Systems, Man and Cybernetics, 36(5):862875 .

Johanson, U. and Johraon, A. (2001). Personalekonomi idag. Technical report, Uppsala Publishing House,Uppsala, Sweden.

Johns, G. (2010). Presenteeism in the workplace: A review and research agenda. Journal of Organizational Behavior, 31:519-542.

Karasek, R., Brisson, C., Kawakami, N., Irene Houtman, I., Bongers, P., and Amick, B. (1998). The job content questionnaire (jcq): An instrument for internationally comparative assessments of psychosocial job characteristics. Journal of Occupational Health Psychology, $3(4): 322-355$.

Katou, A. and Budhwar, P. (2012). Causal relationship between hrm policies and organisational performance: Evidence from the greek manufacturing sector. European Management Journal, 28(1):25-39.

Kennedy, C., Amick, B., Dennerlein, J., Brewer, S., Catli, S., Williams, R., Serra, C., and Gerr, F. (2010). Systematic review of the role of occupational health and safety interventions in the prevention of upper extremity musculoskeletal symptoms, signs, disorders, injuries, claims and lost time. Journal of Occupational Rehabilitation, 20(2):127-162. 
Kerr, M. (1997). A case-control study of biomechanical and psychosocial risk factors for low back pain reported in an occupational setting. PhD thesis, University of Toronto.

Kerr, M. (1998). Workplace psychosocial factors and musculoskeletal disorders: A discussion paper. Technical report, Institute for Work \& Health, Toronto, Ontario.

Kerr, M., Frank, S. S., Norman, J., Wells, R. W. K., and Neumann, W. (2001). Biomechanical and psychosocial risk factors for low back pain at work. American Journal of Public Health, 91(7):1069-1075.

Kerst, J. (2003). An ergonomics process for the care and use of research animals. Institute for Laboratory Animal Research Journal, 44(1):3-12.

King, G. and Zeng, L. (2002). Estimating risk, rate levels, ratios and different risks in casecontrol studies. Statistics in Medicine, 21:1409-1427.

Kleinbaum, D. G. and Klein, M. (2010). Logistic Regression. New York, NY : Springer New York.

Kohlmorgen, U., Schmeck, H., and Haase, K. (1999). Experience with fine-grained parallel genetic algorithms. Annals of Operations Research, 90:203-219.

Konz, S. (1998). Work/rest: Part ii - the scientific basis (knowledge base) for the guide. International Journal of Industrial Ergonomics, 22(1-2):73-99.

Kopardekar, P. and Mital, A. (1994). The effect of different work-rest schedules on fatigue and performance of a simulated directory assistance operator's task. Ergonomics, 10:1697-1707.

Kucera, K., Loomis, D., Lipscomb, H. J., Marshall, S.W. and, G. M., and Daniels, J. (2009). Ergonomic risk factors for low back pain in north carolina crab pot and gill net commercial fishermen. American Journal of Industrial Medicine, 52(4):311-321.

Kuijera, P. F., Hoozemans, M. J., and Frings-Dresen, M. H. (2007). A different approach for the ergonomic evaluation of pushing and pulling in practice. International Journal of Industrial Ergonomics, 37:855-862. 
Kulasingam, S. and Myers, E. (2003). Potential health and economic impact of adding a human papillomavirus vaccine to screening programs. The Journal of American Medical Association, 290(6):781-789.

Kumar, R. and Kumar, S. (2008). Musculoskeletal risk factors in cleaning occupation-a literature review. International Journal of Industrial Ergonomics, 38(2):158-170.

Laaksonen, M., Pitkaniemi, J., Rahkonen, O., and Lahelma, E. (2010). Work arrangements, physical working conditions, and psychosocial working conditions as risk factors for sickness absence: Bayesian analysis of prospective data. Annals of Epidemiology, 20(5):332-338.

Lahiri, S., Markkanen, P., and Levenstein, C. (2005). The cost effectiveness of occupational health interventions: Preventing occupational back pain. American Journal of Industrial Medicine, 48(6):515-529.

Lanoie, P. and Tavenas, S. (1996). Costs and benefits of preventing workplace accidents: the case of participatory ergonomics. Safety Science, 24(3):181-196.

Larsson, B., Sogaard, K., and Rosendal, L. (2007). Work related neck-shoulder pain: A review on magnitude, risk factors, biochemical characteristics, clinical picture and preventive interventions. Best Practice and Research Clinical Rheumatology, 21(3):447-463.

Leigh, G., Waehrer, M., and Keenan, C. (2004). Costs of occupational injury and illness across industries. Scandinavian Journal of Work, Environment and Health, 30(3):199-205.

Leigh, J. (2011). Economic burden of occupational injury and illness in the united states. The Milbank Quarterly, 89(4):728-772.

Leigh, J. P., Markowitz, S. B., Fahs, M., Shin, C., and Landrigan, P. J. (1997). Occupational injury and illness in the united states: Estimates of costs, morbidity, and mortality. Archives of Internal Medicine, 157(14):1557-1568.

Lerner, D., Amick, B., Lee, J., Roone, T., Rogers, W., Chang, H., and Berndt, E. (2003). Relationship of employee-reported work limitations to work productivity. Medical care, 41(5):649659. 
Lerner, D., Chang, H., Rogers, W. H., Benson, C., Schein, J., and Allaire, S. (2009). A method for imputing the impact of health problems on at-work performance and productivity from available health data. Journal of Occupational and Environmental Medicine, 51(5):515-524.

Leung, A. S., Chan, C., Ng, J., and Wong, P. (2006). Factors contributing to officers' fatigue in high-speed maritime craft operations. Applied Ergonomics,, 37(5):565-576.

Leung, S. C. and Chan, S. S. (2009). A goal programming model for aggregate production planning with resource utilization constraint. Computers and Industrial Engineering, 56(3):1053 -1064 .

Liang, T. (2007). Application of interactive possibilistic linear programming to aggregate production planning with multiple imprecise objectives. Production Planning and Control, $187: 328-341$.

Lin, C. C., Haas, M., Maher, C. G., Machado, L., and Van Tulder, M. (2011). Cost-effectiveness of general practice care for low back pain: A systematic review. European Spine Journal, 20(7):1012-1023.

Lindeberg, S., Maria, R., BongKyoo, C., Catarina, C., Sven-Olof, I., Robert, K., and Austergren, P.-O. (2011). Psychosocial working conditions and exhaustion in a working population sample of swedish middle-aged men and women. European Journal of public health, 21(2):190196.

Linhard, J. (2005). Understanding the return on health, safety and environmental investments. Safety Research, 36(3):257-260.

Liu, H., Hwang, S. L., and Liu, T. H. (2009). Economic assessment of human errors in manufacturing environment. Safety Science, (47):170-182.

Lockett, A. and Thompson, S. (2001). The resource-based view and economics. Journal of Management, 27(6):723-754.

Long, M. H., Johnson, V., and Bogssian, F. (2012). Work-related upper quadrant musculoskeletal disorders in midwives, nurses and physicians: A systematic review of risk factors and functional consequences. Applied Ergonomics, 43:455-467. 
Lotters, F., Meerding, W.-J., and Burdorf, A. (2005). Reduced productivity after sickness absence due to musculoskeletal disorders and its relation to health outcomes. Scandinavian Journal of Work, Environment Health, 31(5):367-374.

Ma, L., Chablat, D., Bennis, F., and Zhang, W. (2009). A new simple dynamic muscle fatigue model and its validation. International Journal of Industrial Ergonomics, 39(1):211-220.

MacFarlane, G.J. Hunt, I. and Silman, A. (2000). Role of mechanical and psychosocial factors in the onset of forearm pain: Prospective population based study. British Medical Journal, 321:676-679.

MacKenzie, 1. J., Morris, J. A., Jurkovich, G. J., Yasui, Y., Cushing, B. M., Burgess, A. R., DeLateur, B. J., McAndrew, M. P., and Swiontkowski, M. F. (1998). Return to work following injury: The role of economic, social, and job-related factors. American Journal of Public Health, 88(11):1630-1637.

MacLeod, I. (2003). Real-world effectiveness of ergonomic methods. Applied Ergonomics, $34(5): 465-477$.

Mallis, M. M., Mejdal, S., Nguyen, T. T., and Dinges, D. F. (2004). Summary of the key features of seven biomathematical models of human fatigue and performance. Aviation, Space, and Environmental Medicine, 75:4-14.

Mannion, A. F., Horisberger, B., Eisenring, C., Tamcan, O., Elfering, A., and Mailler, U. (2009). The association between beliefs about low back pain and work presenteeism. Journal of Occupational and Environmental Medicine, 51(11):1256-1266.

Marcus, M., Gerr, F., Monteilh, C., Ortiz, D. J., Gentry, E., Cohen, S., Edwards, A., Ensor, C., and Kleinbaum, D. (2002). A prospective study of computer users: Postural risk factors for musculoskeletal symptoms and disorders. American Journal of Industrial Medicine, $41(4): 236-249$.

Marshall, V. (1996). Issues of an aging workforce in a changing society: Cases and comparisons. Toronto: Centre for Studies of Aging, University of Toronto and CARNET. 
Martimo, K.-P., Shiri, R., Miranda, H., Ketola, R., Varonen, H., and Viikari-Juntura, E. (2009). Self-reported productivity loss among workers with upper extremity disorders. Scandinavian Journal of Work, Environment and Health, 35(4):301-308.

Matsubayashi, N. Ishii, Y. W. K. and Yamada, Y. (2009). Full-line or specialization strategy? the negative effect of product variety on product line strategy. European Journal of Operational Research, 196(2):795-807.

Matsudaira, K., Palmer, K., Reading, I., Hira, M., Yoshimura, N., and Coggon, D. (2011). Prevalence and correlates of regional pain and associated disability in japanese workers. Occupational and Environmental Medicine, 68:191-196.

McTernan, W., Dollard, M., and LaMontagne, D. (2013). Depression in the workplace: An economic cost analysis of depression-related productivity loss attributable to job strain and bullying. Work and Stress, 27(4):321-338.

Meerding, W., Ljzelenberg, W., Koopmanschap, M., Severens, J., and Burdorf, A. (2005). Health problems lead to considerable productivity loss at work among workers with high physical load jobs. Journal of Clinical Epidemiology, 58(5):517-523.

Mendoza, A. and Venturab, J. A. (2010). A serial inventory system with supplier selection and order quantity allocation. European Journal of Operational Research, 207(3):1304-1315.

Meybodi, M. and Foote, B. (1995). Hierarchical production planning and scheduling with random demand and production failure. Annals of Operations Research, 59(1):259-280.

Meyr, H. (2000). Simultaneous lotsizing and scheduling by combining local search with dual re-optimization. European Journal of Operational Research, 120:311-326.

Michalos, G., Makris, S., and Chryssolouris, G. (2013). The effect of job rotation during assembly on the quality of final product. $\{C I R P\}$ Journal of Manufacturing Science and Technology, 6(3):187 - 197.

Mirzapour Al-e hashema, S., Maleklyb, H., and Aryanezhada, M. (2011). A multi-objective robust optimization model for multi-product multi-site aggregate production planning in a supply chain under uncertainty. Journal of Production Economics, 134(1):28-42. 
Mock, V., Pickett, M., Ropka, M. E., Lin, E. M., Stewart, K. J., Rhodes, V. A., McDaniel, R., Grimm, P. M., Krumm, S., and McCorkle, R. (2001). Fatigue and quality of life outcomes of exercise during cancer treatment. Cancer Practice, 9(3):119-127.

Moon, S. and Sauter, S. (2005). Beyond Biomechanics: Psychosocial aspects of musculoskeletal disorders in office work. Taylor and francis Ltd.

Morse, M., Kros, J. F., and Nadler, S. S. (2009). A decision model for the analysis of ergonomic investments. International Journal of Production Research, 47(21):6109-6128.

Most, D. and Keles, D. (2010). A survey of stochastic modelling approaches for liberalised electricity markets. European Journal of Operational Research, 207:543-556.

Murray, C. and Lopez, A. (1996). Evidence-based health policy-lessons from the global burden of disease study. Science, 274(5288):740-743.

Myszewski, J. M. (2010). Mathematical model of the occurrence of human error in manufacturing processes. Quality and Reliability Engineering International, (26(8)):845-851.

National Research Council (2001). Musculoskeletal Disorders and the Workplace: Low Back and Upper Extremities. National Academy Press, Washington, DC.

Neidigh, R. O. and Harrison, T. P. (2010). Optimising lot sizing and order scheduling with non-linear production rates. International Journal of Production Research, 48(8):2279-2295.

Neumann, W. P. (2004). Production Ergonomics: Identifying and managing risk in the design of high performance work systems. PhD thesis, Department of Design Science, Lund Technical University, Sweden.

Neumann, W. P. and Dul, J. (2010). Human factors: Spanning the gap between om and hrm. International Journal of Operations $\&$ Production Management, 30(9):923-950.

Neumann, W. P., Ekman, E., and Winkel, J. (2009). Integrating ergonomics into production system development: The volvo powertrain case. Applied Ergonomics, 40(3):527-537.

Neumann, W. P., Wells, R., and Norman, R. (1999). 4d-watbak: Adapting research tools and epidemiological findings to software for easy application by industrial personnel. Proceedings of the International Conference on Computer-Aided Ergonomics and Saftey, Barcelona, Spain. 
NIOSH (1993). Heta report 90-013-2277 Los Angeles CA.

Nishii, L. H., Lepak, D. P., and Schneider, B. (2008). Employee attribution of the why of hr practices: Their effects on employee attitudes and behaviors, and customer satisfaction. Personnel Psychology, 61(3):503-545.

Norman, R., Wells, R., Neumann, W. P., Frank, J., Shannon, H., and Kerr, M. (1998). A comparison of peak vs cumulative physical work exposure risk factors for the reporting of low back pain in the automotive industry. Clinical Biomechanics, 13(8):561 - 573.

Novoa, C. and Storer, R. (2009). An approximate dynamic programming approach for the vehicle routing problem with stochastic demands. European Journal of Operational Research, 196(2):509-515.

Nurminen, M. and Karjalainen, A. (2001). Epidemiologic estimate of the proportion of fatalities related to occupational factors in finland. Scandinavian Journal of Work, Environment and Health, 27(3):161-213.

Oh, I. H., Seo, H. H., Kim, E. J., and Kim, Y. A. (2011). The economic burden of musculoskeletal disease in korea: A cross sectional study. BMC Musculoskeletal Disorders, 12(157):14712474 .

Omar, M., Mears, L., Kurfess, T., and Kiggans, R. (2011). Organizational learning in automotive manufacturing: a strategic choice. Journal of Intelligent Manufacturing, 22(5):709-715.

Oxenburgh, M. and Marlow, P. (2005). The productivity assessment tool: Computer-based cost benefit analysis model for the economic assessment of occupational health and safety interventions in the workplace. Journal of Safety Research, 36(3):209-214.

Oxenburgh, M., Marlow, P., and Oxenburgh, A. (2004). Increasing productivity and profit through health and safety: The financial returns from a safe working environment. CRC Press, Bocka Raton, Fla.

Ozdamar, L. and Bozyel, M. (2000). The capacitated lot sizing problem with overtime decisions and setup times. IIE Transactions, 32:1043-1057. 
Panda, D., Kar, S., Maity, K., and Maiti, M. (2008). A single period inventory model with imperfect production and stochastic demand under chance and imprecise constraints. European Journal of Operational Research, 188:121-139.

Parhizi, S. Steege, l. and Pasupathy, K. (2013). Mining the relationships between psychosocial factors and fatigue dimensions among registered nurses. International Journal of Industrial Ergonomics, 43:82-90.

Pavitsos, A. and Kyriakidis, E. (2009). Markov decision models for the optimal maintenance of a production unit with an upstream buffer. Computers and Operations Research, 36(6):19932006.

Penn, M. and Raviv, T. (2006). Optimizing the quality control station configuration. Family Practice, 54(3):301-3014.

Perlin, L. and Schooler, C. (1978). The structure of coping. Journal of Health and Social Behavior, 19:2-21.

Pochet, Y. and Wolsey, L. (1988). Lot-size models with backlogging: Strong reformulations and cutting planes. Mathematical Programming, 40(1):317-335.

Prado-Lu, D. and Leilanie, J. (2004). Risk factors to musculoskeletal disorders and anthropometric measurements of filipino manufacturing workers. International Journal of Occupational Safety and Ergonomics, 10(4):349-359.

Punnett, L. and Wegman, D. (2004). Work-related musculoskeletal disorders: the epidemiologic evidence and the debate. Journal of Electromyography and Kinesiology, 14(1):13-23.

Qin, R. and Nembhard, D. A. (2012). Demand modeling of stochastic product diffusion over the life cycle. International Journal of Production Economics, 137(2):201-210.

Ramond, A., Bouton, C., Richard, I., Roquelaure, Y., Baufreton, C., and Legrand, E. (2011). Participatory ergonomics to reduce exposure to psychosocial and physical risk factors for low back pain and neck pain: Results of a cluster randomised controlled trial. Family Practice, $28(1): 12-21$. 
Ramond, A., Bouton, C., Richard, I., Roquelaure, Y., Baufreton, C., Legrand, E., and Huez, J.-F. (2010). Psychosocial risk factors for chronic low back pain in primary care: A systematic review. Family Practice, 28(1):12-21.

Riel, P. and Imbeau, D. (1998). How to properly allocate the health and safety insurance cost within the firm. Safety Research, 29(1):25-34.

Rijn, R., Huisstede, B., Koes, B., and Burdorf, A. (2010). Associations between work-related factors and specific disorders of the shoulder:a systematic review of the literature. Scandinavian Journal of Work, Environment \& Health, 36(3):189-201.

Rivilis, I., Van Eerd, D., Cullen, K., Cole, D., Irvin, E., Tyson, J., and Mahood, Q. (2008). Effectiveness of participatory ergonomic interventions on health outcomes: A systematic review. Applied Ergonomics, 39(3):342-358.

Rose, L. (2001). Models and methods for analysis and improvement of physical work environments. PhD thesis, Dep. of Product and Productions Development, Div. of Human Factors Engineering, Chalmers University of Technology, Gouteborg, SE.

Rose, L., Orrenius, U., and Neumann, W. (2013). Work environment and the bottom line: Survey of tools relating work environment to business results. Human Factors and Ergonomics in Manufacturing and Service Industries, 23:368-381.

Ross, S. (2003). Introduction to probability models. Academic Press.

Ruckart, P. Z. and Burgess, P. A. (2007). Human error and time of occurrence in hazardous material events in mining and manufacturing. Journal of Hazardous Materials, 142:747-753.

Sajadieh, M., Akbari Jokar, M., and Modarres, M. (2009). Developing a coordinated vendorbuyer model in two-stage supply chains with stochastic lead-times. Computers and Operations Research, 36(8):2484-2489.

Schulte, P. (2005). Characterizing the burden of occupational injury and disease. Journal of Occupational and Environmental Medicine, 47(6):607-622.

Schultz, A. B., Chin-Yu, A. B., Chen, C. Y., and Edington, D. W. (2009). The cost and impact of health conditions on presenteeism to employers. PharmacoEconomics, 27(5):365-378. 
Schultz, A. B. and Edington, D. W. (2007). Employee health and presenteeism: A systematic review. Journal of Occupational Rehabilitation, 17:547-579.

Schultz, K. L., Schoenherr, T., and Nembhard, D. (2010). An example and a proposal concerning the correlation of worker processing times in parallel tasks. Management Science, 56(1):176191.

Seeley, P. and Marklin, R. (2003). Business case for implementing two ergonomic interventions at an electric power utility. Applied Ergonomics, 34(5):429-439.

Shang, K. H. (2012). Single-stage approximations for optimal policies in serial inventory systems with nonstationary demand. Manufacturing $\&$ Service Operations Management, 14(3):414422.

Siddharthan, K., Nelson, A., and Weisenborn, G. (2005). A business case for patient care ergonomic interventions. Nursing Administration Quarterly, 29(1):63-71.

Silverstein, B., Viikari-Juntura, E., and Kalat, J. (2002). Use of a prevention index to identify industries at high risk for work-related musculoskeletal disorders of the neck, back, and upper extremity in washington state, 1990-1998. American Journal of Industrial Medicine, 41(3):149-169.

Sim, J., Lacey, R., and Lewis, M. (2006). The impact of workplace risk factors on the occurrence of neck and upper limb pain: a general population study. British Public Health, 6:234-244.

Slack, N. (2005). The flexibility of manufacturing systems. International Journal of Operations E Production Management, (25(12)):1190-1200.

Sterman, J. (2000). Business dynamics: systems thinking and modeling for a complex world. Irwin/McGraw-Hill, Boston.

Stewart, W., Ricci, J., Chee, E., Morganstein, D., and Lipton, R. (2003). Lost productive time and cost due to common pain conditions in the us workforce. The Journal of the American Medical Association, 290(18):2443-2454. 
Sun, L., Paez, O., Lee, D., Salem, S., and Daraiseh, N. (2006). Estimating the uninsured costs of work-related accidents, part i: a systematic review. Theoretical Issues in Ergonomics Science, $7(3): 227-245$.

Tang, K., Beaton, D., Amick, B., Hogg-Johnson, S., Cote, P., and Loisel, P. (2013). Confirmatory factor analysis of the work limitations questionnaire (wlq-25) in workers compensation claimants with chronic upper-limb disorders. Journal of Occupational Rehabilitation, $23(2): 228-238$.

Tang, O. (2004). Simulated annealing in lot sizing problems. International Journal of Production Economics, 88(2):173-181.

Tehrani Nejad Moghaddam, A. and Michelot, C. (2009). A contribution to the linear programming approach to joint cost allocation: Methodology and application. European Journal of Operational Research, 197(3):999-1011.

Tompa, E., Culyer, A., and Dolinschi, R. (2008). Economic evaluation of interventions for occupational health and safety - developing good practice. Oxford Press, Oxford.

Tsou, C. (2009). Evolutionary pareto optimizers for continuous review stochastic inventory systems. European Journal of Operational Research, 195(2):364-371.

Tuncel, S., Genaidy, A., Shell, R., Salem, S., Karwowski, W., Darwish, M., Noel, F., and Singh, D. (2008). Research to practice: Effectiveness of controlled workplace interventions to reduce musculoskeletal disorders in the manufacturing environment. Human Factors and Ergonomics in Manufacturing, 18(2):93-124.

Van Hop, N. (2007). Fuzzy stochastic goal programming problems. European Journal of Operational Research, 176(1):77-86.

Vandergrift, J. L., Gold, J. E., Hanlon, A., and Punnett, L. (2012). Physical and psychosocial ergonomic risk factors for low back pain in automobile manufacturing workers. Occupational and Environmental Medicine, 69:29-34.

Vieira, E. and Kumar, S. (2007). Occupational risks factors identified and interventions suggested by welders and computer numeric control workers to control low back disorders in two steel companies. International Journal of Industrial Ergonomics, 37(6):553-561. 
Wahab, M. I .M. Wu, D. and Lee, C. G. (2008). A generic approach to measuring the machine flexibility of manufacturing systems. European Journal of Operational Research, 186(1):137149.

Wahab, M. I. M. and Jaber, M. (2010). Economic order quantity model for items with imperfect quality, different holding costs, and learning effects: A note. Computers and Industrial Engineering, 58(1):186-190.

Wahab, M. I. M. and Lee, C. G. (2009). A lattice approach to pricing of multivariate contingent claims with regime switching. Journal of Derivatives, 17(1):49-61.

Wahab, M. I. M. and Lee, C. H. (2011). Pricing swing options with regime switching. Annals of Operations Research, 185(1):139-160.

Wahab, M. I. M., Yin, Z., and Edirisinghe, N. (2010). Pricing swing options in the electricity markets under regime-switching uncertainty. Quantitative Finance, 10(9):975-994.

Wan, W., Hoskisson, R., Short, J., and Yiu, D. (2011). Resource-based theory and corporate diversification: Accomplishments and opportunities. Journal of Management, 37(5):13351368.

Wang, K., Hsu, F., and Liu, P. (2002). Modeling the bathtub shape hazard rate function in terms of reliability. Reliability Engineering \& System Safety, 75(3):397-406.

Wang, R. and Liang, T. (2005). Applying possibilistic linear programming to aggregate production planning. International Journal of Production Economics, 98(3):328-341.

Ware, J., Kosinski, M., and Dewey, J. (2000). How to Score Version Two of the SF-36 Health Survey. Lincoln, RI: QualityMetric Incorporated.

Waters, T. R., Putz-Anderson, V., Garg, A., and Fine, L. J. (1993). Revised niosh equation for the design and evaluation of manual lifting tasks. Ergonomics, 36(7):749-776.

Westgaard, R. and Winkel, J. (1997). Review article ergonomic intervention research for improved musculoskeletal health: A critical review. International Journal of Industrial Ergonomics, 20:463-500. 
WHO (1999). Fact sheet 84: Occupational health. ethically correct. economically sound: World health organization, geneva. Technical report, www.who.int/en/.

Winkel, J. and Mathiassen, S. (1994). Assessment of physical work load in epidemiologic studies: Concepts, issues and operational considerations. Ergonomics, 37(6):979-988.

Winston, W. (2004). Operations Research; Applications and Algorithms. Duxbury Press, London.

Wright, P., Dunford, B., and Snell, S. (2001). Human resources and the resource based view of the firm. Journal of Management, 27(6):701-721.

WSIB (2006). Technical report, Workplace Safety and Insurance Board of Ontario, Canada, www.wsib.on.ca.

WSIB (2010a). Technical report, Workplace Safety and Insurance Board of Ontario, Canada: annual report www.wsib.on.ca.

WSIB (2010b). Technical report, Workplace Safety and Insurance Board of Ontario, Canada www.wsib.on.ca.

WSIB (2011). Technical report, Workplace Safety and Insurance Board of Ontario, Canada www.wsib.on.ca.

Xia, T., Xi, L., Zhou, X., and Lee, J. (2012). Dynamic maintenance decision-making for series-parallel manufacturing system based on mam-mtw methodology. European Journal of Operational Research, 221:231-240.

Xie, J. and Dong, J. (2002). Heuristic genetic algorithms for general capacitated lot-sizing problems. Computers and Mathematics with Applications, 44(1-2):263-276.

Xie, M. and Lai, C. D. (1996). Reliability analysis using an additive weibull model with bathtub-shaped failure rate function. Reliability Engineering 8 System Safety, 52(1):87-93.

Yeow, P. H. and Sen, R. N. (2003). Quality, productivity, occupational health and safety and cost effectiveness of ergonomic improvements in the test workstations of an electronic factory. International Journal of Industrial Ergonomics, 32(3):147-163. 
Yeow, P. H. and Sen, R. N. (2006). Productivity and quality improvements, revenue increment, and rejection cost reduction in the manual component insertion lines through the application of ergonomics. International Journal of Industrial Ergonomics, 36(4):367-377.

Zhang, Z., Way Li, K., Zhang, W., Ma, L., and Chen, Z. (2012). Muscular fatigue and maximum endurance time assessment for male and female industrial workers. International Journal of Industrial Ergonomics, 44(2):292-297. 


\section{Indices, Parameters and Variables}

$j$ - Workstations involved in production $j=1, \ldots, 4$

$y$ - Number of risk factors $y=1,2, \ldots, \mathrm{n}$

$h$ - Being healthy

$w$ - Being in-pain at work

$a$ - Absent from work due to work-related health problems

$p$ - Having pain/injury with productivity loss (employed for the 2 state model)

$\tau$ - Optimization time-period, $\tau=1,2, \ldots, \tau$

$t$ - Time

$r$ - Regular time working

$o$ - Over-time working

$\bar{h}$ - An operator's health-state: $\bar{h}=\{h, w, a, p\}$

$g$ - Growth regime

$d$ - Decay regime

$q$ - Level of the underlying variable in the lattice

$v_{j t}^{\bar{h}}=$ Logistic output with the respect to an operator's health state in station $j$

$u_{j t}^{\bar{h}}=$ Auto-correlation used for the Markov chain of an operator in station $j$ during a year

$\alpha_{0 j}=$ Constant coefficient in the mathematical model for an operator in station $j$

$\beta_{n j}=$ Correlation coefficient of the $n$th WIH risk factor for an operator in station $j$

$\lambda_{j t}^{\bar{h}}=$ Rate of entering to the $\bar{h}$ state from the healthy state for an operator in station $j$

during a year, $\bar{h}=\{h, w, a)$

$\mu_{j t}^{\bar{h}}=$ Rate of entering to the healthy state from the $\bar{h}$ state for an operator in station $j$

during a year, $\bar{h}=\{h, w, a\}$

$\gamma_{j \tau}^{\bar{h}}=3$-state transition rate of entering from one health state to another for an operator

in station $j, \bar{h}=\{h, w, a\}$

$\Lambda_{j t}=$ Proportion of workers reporting pain used for an operator in station $j$

$\pi_{j \tau}^{\bar{h}}=$ Steady-state probability of being in $\bar{h}$ state for an operator in station $j$

$p_{j t}^{\bar{h}}=$ Probability of making errors of an operator in station $j$ and in $\bar{h}$ state

$p_{j t}^{\bar{h} r w}=$ Probability of rework products for an operator in station $j$ and in $\bar{h}$ state

$p_{j t}^{\bar{h} s}=$ Probability of scrap products for an operator in station $j$ and in $\bar{h}$ state 
$\kappa=$ Location parameter of the Weibull distribution

$\zeta_{j}^{\bar{h}}=$ Slope parameter of the Weibull distribution for $\bar{h}$ state

$\rho_{j}^{\bar{h}}=$ Scale parameter of the Weibull distribution for $\bar{h}$ state

$F_{j t}^{w}=$ Cumulative excessive fatigue of the "in-pain" state operator in station $j$

$\Theta_{j t}^{w}=$ Workload level of the "in-pain" state operator in station $j$

$c_{j \tau}^{r}=$ Unit production cost of station $j$ during regular time $\tau$, including labor cost

$c_{j \tau}^{o}=$ Unit production cost of station $j$ during over-time $\tau$, including labor cost

$c s_{j \tau}=$ Unit scraping cost of station $j$ during $\tau$

$c r_{j \tau}=$ Unit reworking cost of station $j$ during $\tau$

$b_{j \tau}=$ Unit inventory cost of the buffer of station $j$ during $\tau$

$\mathbf{B}_{\tau}=$ Inventory level of workstations

$b m_{j \tau}=$ Upper bound capacity of the buffer in station $j$

$\mathbf{I}_{\tau}=$ All possible inventory levels

$h c_{j}=$ Unit cost of changing buffer capacity of station $j$

$s_{\tau}=$ Unit subcontracting cost of outsourcing production during time $\tau$

$z_{j \tau}^{\bar{h}}=$ Average production rate (product/hour) for an operator in station $j$ with $\bar{h}$ state

$d_{\tau}=$ External demand in time $\tau$ period

$m e_{\tau}=$ Average daily medical treatment and compensation expenses

$i n w_{j}=$ Annual insurable salary of an operator in station $j$

$s h_{j \tau}^{r}=$ Number of hours in regular work-shift per day for an operator station $j$

$s h_{j \tau}^{o}=$ Number of hours in over-time work-shift per day for an operator in station $j$

$w l_{j \tau}=$ Average workforce payment rate per hour for station $j$

$m_{j \tau}=$ Number of working days during time $\tau$ period for an operator in station $j$

pct $_{\tau}=$ Percentage of the demand allowed to be provided by subcontractors

$X_{n j}=$ Exposure measure of the $n$th WIH risk factor for an operator in station $j$

$I N=$ Total insurance premium cost of the assembly system

$I C=$ Total indirect OHS costs of the assembly system

$i=$ Annual time value money risk

$\sigma_{g}=$ The volatility value of the growth regime

$\sigma_{d}=$ The volatility value of the decay regime

$\xi_{g}=$ The mean value of the growth regime 
$\xi_{d}=$ The mean value of the decay regime

$k_{\tau}=$ Probability of switching from the growth regime to the decay regime during time $\tau$ $\delta_{(g, u)}=$ Upward branch probability of the growth regime $\delta_{(g, m)}=$ Middle branch probability of the growth regime $\delta_{(g, l)}=$ Downward branch probability of the growth regime $\delta_{(d, u)}=$ Upward branch probability of the decay regime $\delta_{(d, m)}=$ Middle branch probability of the decay regime $\delta_{(d, l)}=$ Downward branch probability of the decay regime

\section{Design Variables:}

$P_{j \tau}^{\bar{h} r}=$ Number of products produced in regular time $\tau$ in $\bar{h}$ state for an operator in station $j$

$P_{j \tau}^{\bar{h} o}=$ Number of products produced in over-time $\tau$ in $\bar{h}$ state for an operator in station $j$

$S_{\tau}=$ Number of products provided by subcontractors during time $\tau$ $B_{j \tau}=$ Number of products stored in the buffer of station $j$ during time $\tau$ 


\section{Acronyms}

HF: Human Factors

HRM: Human Resource Management

IEA: International Ergonomics Association

JCQ: Job Content Questionnaire

NMQ: Nordic Musculoskeletal Questionnaire

NOISH: National Institute for Occupational Safety and Health

OHS: Occupational Health and Safety

MSD: Musculoskeletal Disorders

OM: Operations Management

OMPO: Operations Management Performance Optimization

OR: Operations Research

ROHSEI: Return on Health, Safety and Environmental Investments

TC: Total Cost

TP: Total Production

WE: Working Environment

WIH: Work-related Ill Health 